\title{
Quantifying balance control during stance
}

\section{A multivariate system identification approach}

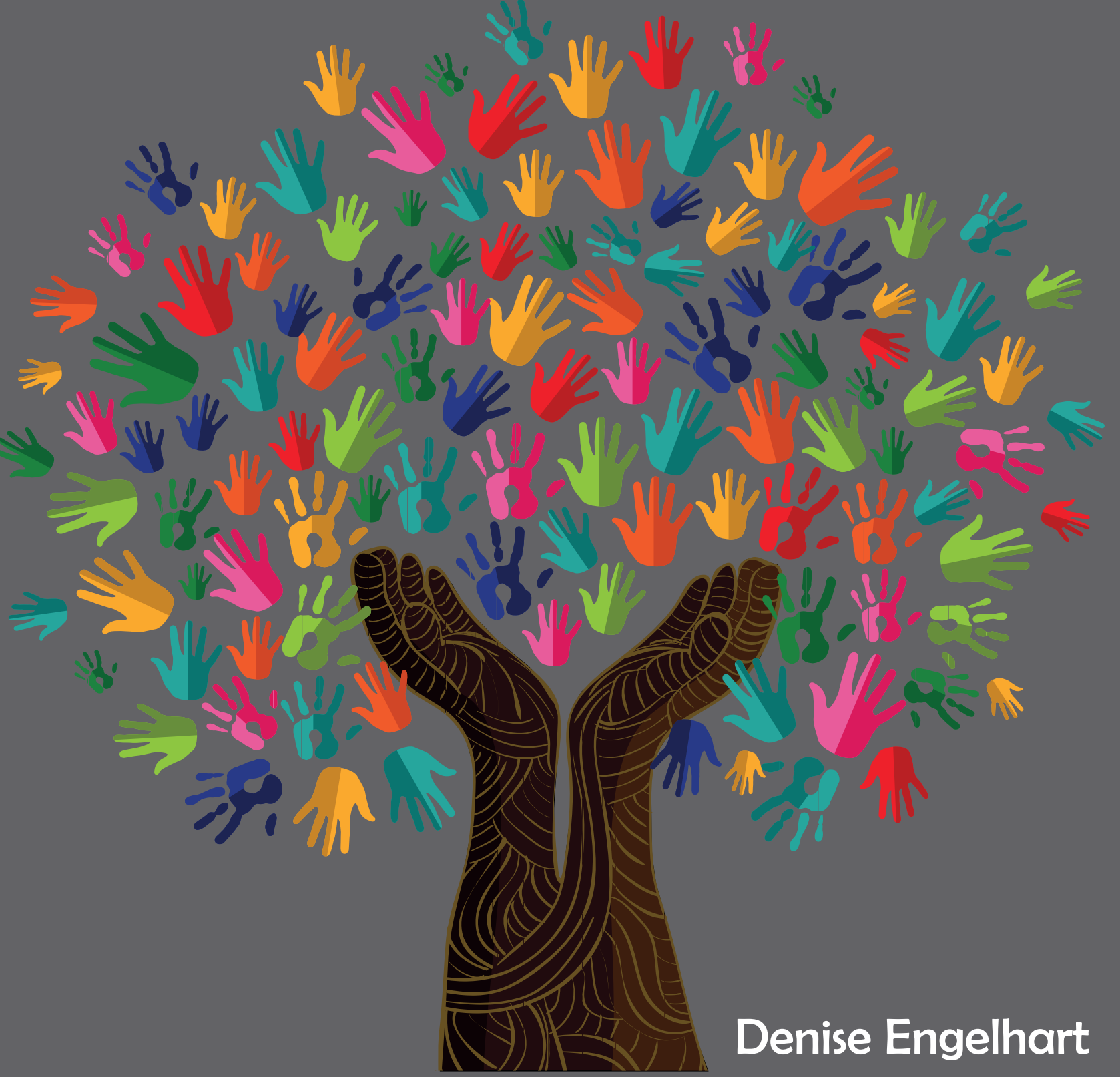


Quantifying balance control during stance:

A multivariate system identification approach

Denise Engelhart 


\section{Samenstelling promotiecommissie}

\section{Voorzitter/secretaris}

prof. dr. G.P.M.R. Dewulf

Promotor

prof. dr. ir. H. van der Kooij

Co-promotoren

dr. ir. A.C. Schouten

dr. ir. R.G.K.M. Aarts

\section{Leden}

prof. dr. ing. W.B. Verwey

prof. dr. ir. P.H. Veltink

prof. dr. J.H. van Dieën

prof. dr. J. Schoukens

dr. R.J. Peterka
Universiteit Twente

Universiteit Twente

Universiteit Twente

Universiteit Twente

Universiteit Twente

Universiteit Twente

Vrije Universiteit Amsterdam

Vrije Universiteit Brussel

Oregon Health \& Science University

This research is supported by the Dutch Technology Foundation STW, which is part of the Netherlands Organisation for Scientific Research (NWO) and partly funded by the Ministry of Economic Affairs (NeuroSIPE \#10737 BalRoom).

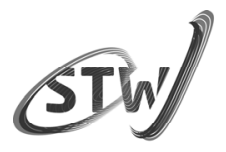

Enabling new technology

Ontwerp omslag en binnenwerk:

Denise Engelhart

\section{Drukwerk:}

Gildeprint - Enschede

ISBN: 978-90-365-3922-7

DOI: $10.3990 / 1.9789036539227$

Copyright @2015 by D. Engelhart, Enschede, the Netherlands

All rights reserved. No part of this publication may be reproduced or transmitted in any form or by any means, electronic or mechanical, including photocopy, recording or any information storage or retrieval system, without permission in writing from the author. 
QUANTIFYING BALANCE CONTROL DRUING STANCE:

A MULTIVARIATE SYSTEM IDENTIFICATION APPROACH

\title{
PROEFSCHRIFT
}

\author{
ter verkrijging van \\ de graad van doctor aan de Universiteit Twente, \\ op gezag van de rector magnificus, \\ prof. dr. H. Brinksma, \\ volgens besluit van het College voor Promoties \\ in het openbaar te verdedigen \\ op donderdag 3 september 2015 om 16:45 uur
}

door

Denise Engelhart

Geboren op 04 december 1986

te Zwolle, Nederland 
Dit proefschrift is goedgekeurd door de promotor prof. dr. ir. H. van der Kooij

en door de co-promotoren

dr. ir. A.C. Schouten

dr. ir. R.G.K.M. Aarts 


\section{Table of Contents}

$\begin{array}{ll}\text { Introduction } & 7\end{array}$

Impaired standing balance in elderly: A new engineering methods helps to unravel causes and effects

Comparison of closed loop system identification methods to quantify multijoint human balance control

Assessment of multi-joint coordination and adaptation in standing balance:

A novel device and system identification technique

Adaptation of multi-joint coordination during standing balance in healthy young and healthy old individuals

Reliability of system identification techniques to assess standing balance in healthy elderly

General Discussion

Bibliography

Summary

143

Samenvatting

147

Acknowledgements

151

About the author 



\section{Chapter 1}

Introduction

Humans are able to maintain balance in many daily life activities and under numerous environmental conditions. Balancing tasks, such as standing, reaching, cycling and walking, seem simple and normally do not require much attention; we are able to keep our posture more or less in the upright position. However, controlling our balance is complex as many muscles, joints, sensory systems and neural pathways are involved to keep our body upright and prevent us from falling. When becoming older, or when suffering from diseases, the balance control system can deteriorate. Elderly people often have difficulties maintaining balance in daily life activities and this impaired balance is a strong risk factor for falls (Rubenstein, 2006; Muir et al., 2010). Specifically, about 28-35\% of people aged over 65 fall each year and this incidence increases with advancing age (WHO, 2007). Falls and fear of falling decreases a person's mobility, influences the quality of life and can even lead to premature death (Ambrose et al., 2013). Although the research field in balance control is large and many studies have been performed on bipedal upright stance, the (patho-) physiology of balance control remains largely unknown. Because the balance control system is redundant, humans can compensate for deficits in one of the balance control mechanisms. For example, in case of unilateral impairment of the muscular system in the lower extremities, people may rely more on their non-affected leg for balancing (van Asseldonk et al., 2006). Due to these compensation mechanisms, especially early-stage balance impairments are hard to detect. Novel methods are required to detect the quality of the underlying mechanisms in standing balance, and to identify the risk factors for falling at an early stage. In this thesis, new methods to identify and quantify standing balance control are presented and evaluated in healthy elderly and healthy younger subjects. This chapter provides the necessary background, formulates the research goal, and provides the outline of the thesis. 


\subsection{Balance control}

Bipedal stance is inherently unstable as the pull of gravity constantly moves the body away from equilibrium, and without a stabilizing control mechanism we would fall. In balance control studies, the body is often represented as an inverted pendulum; i.e. the body is considered as a single mass concentrated in the Centre of Mass (CoM), which rotates around the ankle joint. The ground reaction force represents the weighted pressure average of the area in contact with the ground and exerts at the Centre of Pressure (CoP). During standing balance, the position of the CoP is confined to a limited area, the base of support (BoS), which is roughly the area of the foot.

Balance control is described by the ability of keeping or returning the body CoM over its BoS and depends on three major components, as depicted in Figure 1.1. First the sensory systems, which obtain information about the body position relative to the environment. Secondly, the ability of the brain to process and integrate the information from various sensory systems. Finally, the muscles for coordinating the movements and generation of the corrective joint torques to maintain balance (Peterka, 2003; van der Kooij et al., 2005).

Information about the position and velocity of the body in space comes from three sensory systems; the proprioceptive, visual and vestibular system. Proprioception is the perception of movement and spatial orientation from within the body itself, arising primarily from muscle spindles and Golgi tendon organs.

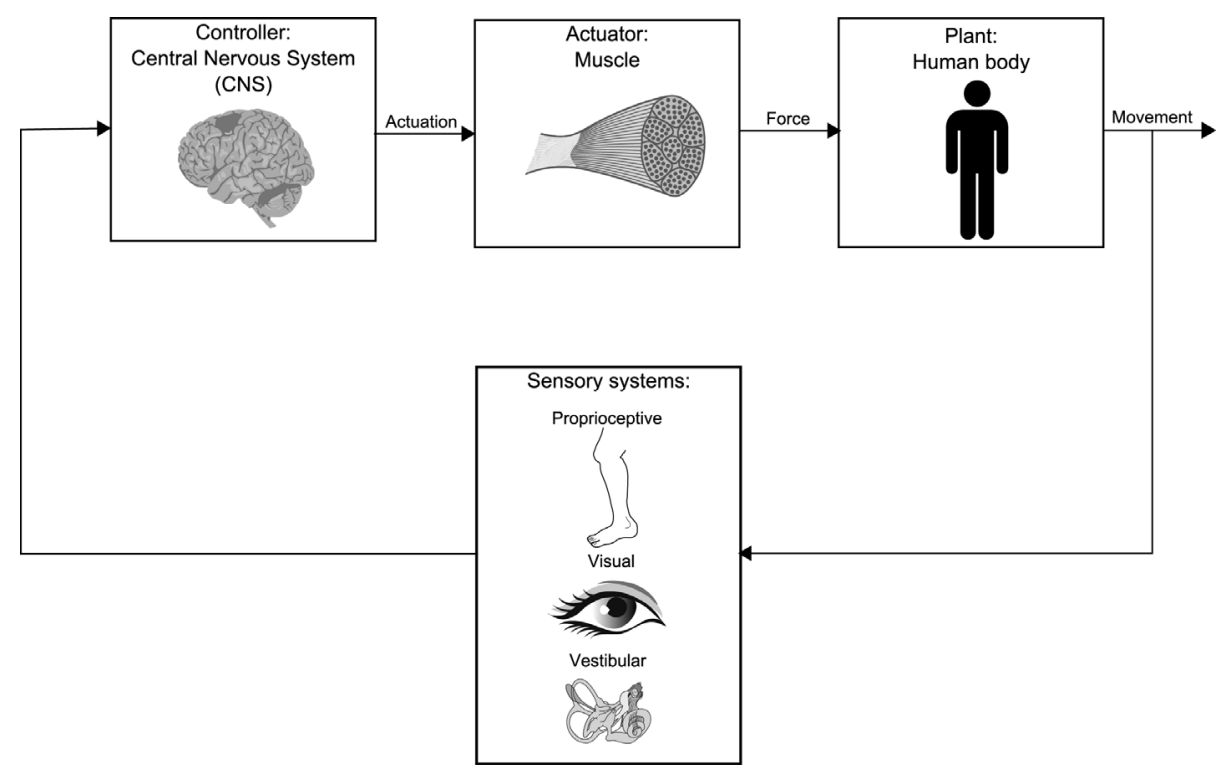

Figure 1.1: A general scheme of human balance control. The proprioceptive, visual and vestibular systems obtain information about body movement relative to the environment. The sensory signals are integrated and processed by the central nervous system which control the muscular activity. Muscle forces result in a corrective joint torque that counteracts the CoM excursion. 
Muscle spindles sense changes in muscle length and contraction velocity. The Golgi tendon organs sense the tension in the tendon, which is directly related to muscle force. The visual system senses the head movement (and velocity) relative to the visual world. The position and translation of the head with respect the gravitational field is sensed by the vestibular organ, which is sensitive to specific translational (otoliths) and rotational (semi-circular channels) accelerations (Mergner, 2010).

To control balance, the information from the sensory systems goes directly to the reflex centres in the brain stem, as the first response to imbalance must be fast and without thinking (i.e. reflexive). The signals are further transported to the cerebellum, where the information is integrated and commands are sent to the brainstem motor control centres where skeletal muscle activity is coordinated and muscle tone is regulated. The activated muscles generate a corrective joint torque which stabilizes the human body (Marieb, 2004). However, during all balancing tasks postural sway is present; i.e. we can never execute a movement perfectly or stand exactly still. This sway results from process noise (execution of movement), measurement noise (associated with sensory information), and computational noise (associated with central processing), which causes the body to sway during normal human upright stance (van der Kooij \& de Vlugt, 2007).

When humans are exposed to external (pushes or pulls on the human body) or internal (noise) disturbances, the best strategy to maintain balance has to be chosen. The body adapts to different circumstances by adjusting the relative contribution of sensory sources in human balance control (the sensory reweighting paradigm (Peterka, 2002)), or using different joint coordination patterns (e.g. ankle and hip strategy (Horak and Nashner, 1986)). In case the adopted strategy becomes insufficient, a fall might occur. Although the general mechanisms for balance control are known, it is not yet fully understood how the different parts of the system work together and interact, or what happens when we become older and systems deteriorate.

\subsection{Ageing and balance dysfunction}

Falling is not uncommon at any age, as learning to stand or walk takes practice and is accompanied with many falls. However with age, the risk of falling increases and especially the consequences of a fall become more severe. Falling can cause profound disruptions in daily life activities.

Aging can affect balance control in various ways. First of all, balance deficits can be caused by a wide range of disorders, including vestibular pathology, visual impairments, neurological diseases and musculoskeletal disorders (Sturnieks et al., 2008; Visser et al., 2008). Secondly, fear of falling may lead to self-imposed restrictions of daily activities and thereby causing immobilization (Bloem et al., 2003; Ambrose et al., 2013). Physical inactivity may lead to a decline in physical capabilities and reduces overall fitness. Reduced physical activity negatively affects postural control, thereby completing a vicious loop. Furthermore, some physiological changes can be related to falls. Carotid sinus syncope is 
increasingly recognized as a common cause of falls in elderly. Also orthostatic hypotension and the reduction of venous pooling in the legs and abdomen by specific maneuvers, such as standing with crossed legs or squatting, can cause falls (Ambrose et al., 2013). Finally, medication can also influence balance control, but the exact influence of medication remains largely unknown (Bloem et al., 2003).

Although the causes of falling are multi-factorial, many epidemiological as well as experimental studies convincingly proved that balance deficits in postural control are the most important risk factors for falls. As the postural system contains many interacting subcomponents, many factors can be underlying the balance deficits (Box 1).

Box 1: Standing balance control requires various resources, which might be altered in elderly. Based on (Horak, 2006)

Biomechanical constraints: Balance control is often defined as the ability to keep the body Centre of Mass (COM) within the base of support (BoS), which is roughly the area of the feet. Therefore, the size of the feet and the touch/pressure sensors of the skin must be of good quality. The distance over which the CoM can move before reaching the edge of the BoS, is called the limit of stability. Elderly people often have smaller limits of stability than young people.

Movement strategies: Three important strategies are used to return the body to equilibrium during stance. In the ankle strategy, the body moves approximately as an inverted pendulum in cases of small disturbances and large support surfaces. Additionally, hip strategy is used when subjects experience large disturbances or narrow support surfaces. If both feet in place strategies are insufficient, a corrective step; e.g. a change of BoS must be made to prevent falling. Additionally, arm movements can be used to control the CoM. When elderly are at risk of falling, they tend to use the hip strategy and stepping strategy more often than young individuals.

Control of dynamics: Controlling balance while changing from one posture to another, or during gait, requires complex control of the CoM. Intersegmental coordination is of importance as the body is not only influenced by external disturbances, but the body segments also affect each other. The available degrees of freedom of the joints and muscle strength contribute to proper control of balance. Furthermore, both forward and lateral stability must be obtained. Elderly tend to have larger lateral excursions of the CoM, and in case of walking their foot placement is more irregular.

Sensory strategies: Three sensory systems determine the position of the body parts with respect to gravity, the support surface, the visual environment and each other. In case of a large and firm support surface and an environment with sufficient light, persons rely on visual $( \pm 10 \%)$, vestibular $( \pm 20 \%)$ and proprioceptive $( \pm 70 \%)$ information. When a person is subject to a changing environment, the contribution of these systems alters; e.g. reliable information is weighted higher than unreliable information (sensory-reweighting). Some disorders (such as cataract or vestibular loss), which might be present in elderly, limit this sensory-reweighting capacity.

Cognitive processing: It is often seen that reaction times and performance in a cognitive task decline when the difficulty of postural task increases; more attention is given to balancing. Falls can result from insufficient cognitive processing to control posture while occupied with a secondary task. 


\subsection{Assessment of balance disorders}

Elderly individuals are falling due to specific, unique constraints on their postural control system (Horak, 2006). For example, an individual who is incapable of using vestibular information will be at risk of falling in a dark environment with a moving support surface. Another individual may be at risk of falling when stepping responses are needed due to improper movement strategies in the ankle and hip.

To assess the quality of balance control in individual patients typically a combination of questionnaires, physical examination, clinical balance tests and posturography techniques are performed (Box 2).

\section{Clinical balance tests}

To make an appropriate differential diagnosis in patients presenting with falls or balance impairments, the clinician typically performs multiple tests. First of all, the case history of the patient is discussed, concerning mobility and falls. Secondly, some cognitive tests are performed; e.g. the Mini Mental State Examination (MMSE, (Folstein et al., 1975)), the Visual Association Test (VAT, (Lindeboom et al., 2002)) or the Montreal Cognitive Assessment (MOCA, (Nasreddine et al., 2005)). Finally, some balance testing protocols are performed in which the clinician scores the patient to perform a motor task in which balance control is involved; e.g. the Short Physical Performance Battery (SPPB, (Guralnik et al., 1994)), the Berg Balance Scale (BBS, (Berg et al., 1989)) or the Timed Up and Go test (TUG, (Podsiadlo and Richardson, 1991)) test.

Although the clinical balance tests are practical in clinical use; i.e. they require simple equipment, they are time efficient and the costs are low, there are some drawbacks. The clinical balance tests all assess a subject's balance, however each test focuses on a different aspect of balance control. Furthermore, these tests have a subjective nature; people tend to forget when and where they have fallen (Cummings et al., 1988), and the scoring system of the balance test can be inconsistent between physicians. The tests are a qualitative assessment of balance disorders; e.g. people are or are not able to maintain balance. However, details of the underlying pathophysiology in individual patients were not unravelled, which hampers the possibility to give targeted therapy on an individual level to prevent falling.

\section{Posturography}

Over the years, several technical developments have occurred that permitted better quantitative studies of the pathophysiology of balance control, like posturography. Posturography techniques are separated into two categories; static and dynamic posturography. In static posturography, balance control is assessed during quiet stance, whereas dynamic posturography involves the use of experimentally induced balance disturbances (Visser et al., 2008).

The way humans correct for balance disturbances during stance can be allocated into three categories: 
Box 2: Existing measurement techniques to assess balance

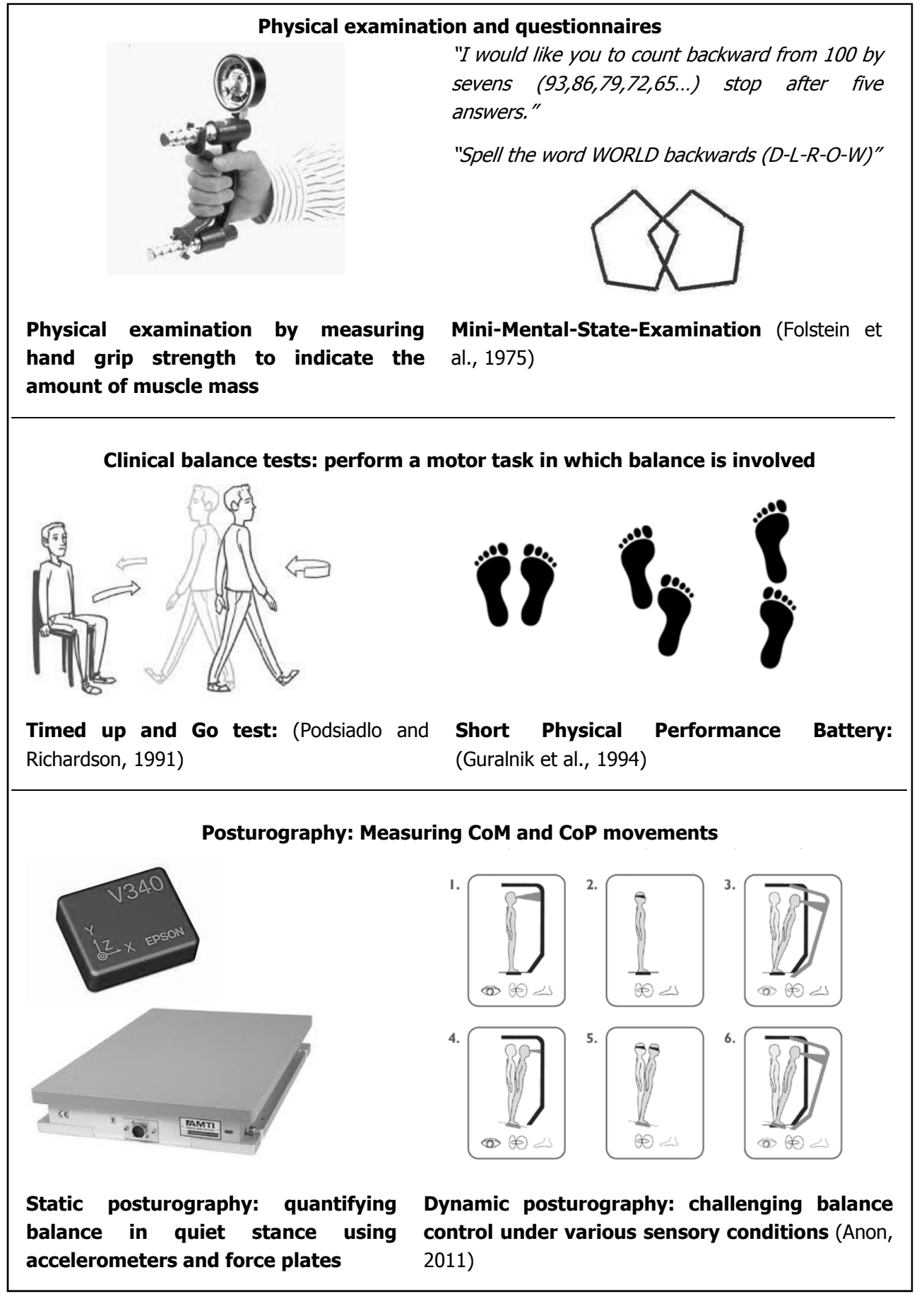


1) Feet in place responses, where the feet remain on the floor and the corrective joint torques around the ankles and hips keep the body in the upright position. It has been show that the ankles mostly correct for small and slow disturbances and the hips correct for faster and larger disturbances (Horak and Nashner, 1986; Creath et al., 2005).

2) In case the ankle and hip torques are not sufficient to control balance, a corrective step is made, controlling the CoM by enlarging the base of support (Weerdesteyn et al., 2012).

3) Using protective arm movements in combination with the two above strategies. Moving the arms decreases the momentum of the CoM and thereby decreases the influence of the disturbance (van Asseldonk et al., 2007).

With posturography techniques, the above described subject's response to balance disturbances are assessed by measuring body sway excursions (with inertial sensors or position tracking systems) and ground reaction forces (with force plates or in-shoe pressure sensors). This makes it possible to detect balance or gait abnormalities in a more quantitative assessment.

In addition to balance responses in stance, posturography methods allow to detect abnormalities in gait pattern, such as asymmetries, decreased walking speed or shuffling gait (Ganz et al., 2007). Furthermore, detection of a deterioration in a specific sensory system can be facilitated by manipulating the base of support (varying foot positions), with eyes open or eyes closed (eliminating visual information), or on a firm or compliant surface (Visser et al., 2008; Pasma et al., 2014b). The NeuroCom is a clinically available device based on dynamic posturography and provides an assessment of the quality of the individual sensory systems. In six sensory conditions, information of vision, vestibular and proprioceptive information is eliminated or disturbed. The ratio's between conditions give an indication of the quality of the sensory systems (Anon, 2011).

A major advantage of using posturography techniques is that combining inertial sensors with in-shoe pressure devices, makes it possible to assess balance and gait outside the laboratory during daily life activities. However, the clinical utility of these posturography methods has been reviewed focusing on several areas where clinicians presently experience the greatest difficulties (Visser et al., 2008; Kingma et al., 2011). It was concluded that none of the existing techniques is currently able to significantly influence the clinical decision making in individual patients due to the limitations in subject selection, data collection, analysis and interpretation of the results. Furthermore, these techniques do not unravel the underlying (patho-)physiological mechanisms in balance control. The contribution of these different mechanisms are difficult to differentiate since they form multiple parallel and nested feedback loops (van der Kooij et al., 2005), and cause and effect relations are intermingled. It is difficult to determine if, for example, changes in electromyography (EMG) activity result in changes in muscle force that will affect body sway, or that the opposite is true, i.e. changes in body sway are detected by sensors and transmitted to the nervous system that excites the muscle groups reflected in EMG changes. Due to this causality 
problem, the impaired underlying mechanism that causes difficulties in balance control cannot be detected.

\section{System identification techniques}

System identification techniques have the potential to unravel cause and effect relations and to detect the impaired underlying mechanism of balance control. Specifically designed external disturbances are used to excite and challenge the balance control system. One can think of force disturbances (pushes or pulls on the human body) or sensory disturbances (providing subjects with additional visual information). These external disturbances provide a unique input that is not related to the internal signals of the system, and create a causal relation between the disturbances and the CoM angle and the corrective ankle torque. By transforming the signals to the frequency domain, the system dynamics is expressed in a frequency response function (FRF). The FRF gives information about the magnitude and timing of the responses to the disturbances as a function of frequency. In addition, a model of the balance control system (in which each underlying mechanism is represented by a mathematical function) can be fitted on the experimentally acquired FRF, to determine physiological relevant parameters such as stiffness, damping, time delays and noise levels. To assess changes in balance control with age or disease, the FRFs and the parameters can be compared between age groups or individuals.

\subsection{NeuroSIPE: BaIRoom project}

Within the NeuroSIPE program, which is supported by the Dutch Technology Foundation STW, system identification and parameter estimation techniques are used in order to develop diagnostic tools for neurological disorders. Within NeuroSIPE program multiple projects exist, each focusing on a specific disorder. Specifically in the BalRoom project, we aim to develop a Balance Test Room (BalRoom) that quantifies balance deficits and unravels the underlying pathophysiological mechanisms in balance control for people with balance disorders. The project is a collaboration between universities of technology, university medical centers and industrial partners. At the University of Twente, the aim is to develop the BalRoom hardware (in collaboration with Motekforce Link B.V.), algorithms and protocols, and to validate the device in a healthy population. A close collaboration exists with the Leiden University Medical Center, to validate the BalRoom under different clinical conditions in a population of elderly with impaired balance. In collaboration with the LUMC and the Centre for Human Drug Research in Leiden, the BalRoom will be validated to detect changes in balance control due to medication (benzodiapine and lorazepam) in healthy elderly. In a later stage work will take place in the Radboud University Nijmegen Medical Centre in Nijmegen, to establish the sensitivity of the BalRoom and the relationship with existing balance scores.

The two main tasks within the BalRoom project at the University of Twente are: the development of BalRoom hardware and the development of novel system identification techniques. 


\section{Development of BalRoom hardware}

To unravel cause and effect in balance control, external disturbances are required, which manipulate specific systems within the closed-loop. The goal of the BalRoom is to apply multiple disturbances to detect the underlying mechanisms in multi-segmental balance control and sensory reweighting capacities. All disturbances should be submaximal (without making people make a step or even fall) and act on the body simultaneously to reduce measurement time.

To apply these disturbances, specifically designed hardware is required. The development of the BalRoom hardware is modular, to test if the application of individual disturbances result in the desired outcome measures. Subsequently, the modules will be combined to study the application of simultaneous disturbances on the balance control system. The first module had to apply disturbances to manipulate proprioceptive information of the legs to study sensory reweighting capacities. This device was developed prior to the BalRoom project; the Bilateral Ankle Perturbator (Schouten et al., 2011). The second module had to apply two independent force disturbances on the pelvis and between the shoulder blades, which should result in upper and lower body movement in the anterior/posterior direction. This allows us to study multi-segmental balance control; i.e. the contribution of the ankles and hips to maintain standing balance. The third module had to apply a visual scene disturbance to manipulate visual information to study sensory reweighting capacities.

\section{Development of system identification techniques}

Based on the experimental data obtained with the BalRoom hardware, system identification techniques can be applied to estimate the underlying mechanisms of balance control. Although existing system identification techniques have added value over posturography, most studies were restricted to application of one disturbance and by simplifying the body as an inverted pendulum. The research goal is to extent system identification techniques to multiple-input multiple-output models, to capture multi-segmental balance control. We will investigate where to perturb the human body, select the most suitable system identification method an fit balance control models on the obtained data to quantify the accuracy of visual, vestibular and proprioceptive information, time delays in reflex loops, mechanical properties of muscles and efficiency of inter-segmental coordination.

\subsection{Thesis outline}

The general goal of this thesis is to create further insight in the (patho-)physiology of standing balance control in elderly. To achieve this goal, three major objectives were defined:

1) To develop novel and improved experimental techniques to assess the contribution of the underlying mechanisms in standing balance control.

2) To quantify age-related changes in standing balance control.

3) To validate the techniques, to quantify the underlying mechanisms of standing balance control in clinical practice. 
The studies that were performed to achieve the research goal and objectives, are described in five chapters (Chapter 2-6), from which the content has been published or submitted as journal articles. In the last chapter (Chapter 7), the applied methods and findings are discussed together with come implications and directions for future research.

Chapter 2 elaborates on the added value of using system identification techniques over clinical balance tests. System identification techniques possibly can detect the impaired underlying mechanisms in balance control and the compensation strategies which are at work. We reviewed current clinical balance tests and described the need for system identification techniques in the diagnostic field.

Chapter 3 compares various system identification techniques for multi-segmental balance control to identify the contribution of the ankles and hips in standing balance. Based on model simulations it was investigated whether these methods were able to reliably estimate the underlying dynamics to maintain upright stance.

Chapter 4 describes the development of a novel experimental set-up, which applies mechanical disturbances at the hip and shoulder level. In combination with system identification techniques, the underlying dynamics of multi-joint coordination in balance control was assessed. Furthermore, we studied adaptation of multi-joint coordination as a response to external force fields.

Chapter $\mathbf{5}$ investigates whether adaptation of multi-joint coordination is different with age. The device and system identification technique, which were presented in Chapter 4, were used in a study with fifteen healthy young and fourteen healthy elderly participants. Fitting a model of balance control on the experimental data was used to detect the underlying physiological changes when becoming older.

Chapter 6 investigates the reliability of standing balance parameters obtained with system identification techniques. The two mechanical disturbances as described in Chapter 4 and Chapter 5 were combined with two sensory (visual and proprioceptive) disturbances. All disturbances were simultaneously applied and the reliability of the frequency response functions was assessed in a group of twelve healthy elderly.

Chapter 7 discusses all findings of this thesis. The use of system identification methods and the results of the studies are critically discussed and future directions are elaborated on. 


\title{
Chapter 2
}

\section{Impaired standing balance in elderly: A new engineering methods helps to unravel causes and effects}

Denise Engelhart, Jantsje H. Pasma, Alfred C. Schouten, Carel G.M. Meskers, Andrea B. Maier, Thomas Mergner and Herman van der Kooij. JAMDA 15 (2014) 227.e1-227.e6

\begin{abstract}
Deteriorated balance control is the most frequent cause of falls and injuries in the elderly. Balance control comprises a complex interplay of several underlying systems (i.e. the sensory systems, the motor system, and the nervous system). Available clinical balance tests determine the patient's ability to maintain standing balance under defined test conditions and aim to describe the current state of this ability. However, these tests do not reveal which of the underlying systems is deteriorated and to what extent, so that the relation between cause and effect often remains unclear. Especially detection of early-stage balance control deterioration is difficult, because the balance control system is redundant and elderly may use compensation strategies. This article describes a new method that is able to identify causal relationships in deteriorated balance control, called CLSIT (Closed Loop System Identification Technique). Identification of impaired balance with CLSIT is a base for development of tailored interventions and compensation strategies to reduce the often serious consequences of deteriorated balance control in the elderly.
\end{abstract}




\subsection{Introduction}

Falls and injuries of the elderly often occur due to impaired balance and may result in decreased quality of life and premature death (Bloem et al., 2003; Sturnieks et al., 2008). In daily life, balance is constantly challenged by disturbances. These may be both external such as uneven ground or pushes having impact on the body - and internal, such as inaccurate sensory or motor functions or, indirectly, impairments of the cardiovascular system with orthostatic hypotension, hypertension, or atrial fibrillation (Ambrose et al., 2013). Maintaining balance depends on the appropriate functioning of the balance control system, which comprises the sensory, nervous, and motor systems. These systems are often directly affected when becoming older, for example by (1) sensory system deteriorations due to poor visual contrast sensitivity and depth perception, impairment of the vestibular self-motion sense, or an inaccurate proprioceptive sense of joint position due to polyneuropathy, (2) nervous system deterioration due to inadequate movement coordination or impaired attention, (3) motor system deterioration due to muscle weakness or paresis, or (4) combinations of these factors (Sturnieks et al., 2008).

Impaired balance often becomes symptomatic when several factors co-occur. On the other hand, there exists some redundancy within the underlying systems, which allows maintaining balance despite of problems in the sensory, nervous or motor systems by using appropriate strategies. For example, when vision is deteriorated, the elderly may rely more on vestibular or proprioceptive information for balance (Peterka, 2002). Or, in case of a unilateral impairment of the muscular system, elderly may use preferentially the nonaffected leg for balancing (van Asseldonk et al., 2006). Therefore, the impairments may initially remain unnoticed and without appropriate therapy. However, when the adopted compensatory strategy becomes insufficient, or the deterioration in the underlying system is too severe, or when the compensating system is also affected, then impaired balance tends to become symptomatic and a fall may occur.

Balance control is often studied during bipedal upright stance, in the following referred to as standing balance. It is the basis for many daily life activities such as walking, reaching and rising from a chair. Standing balance control tries to keep the body's Centre of Mass (CoM) over the base of support, the feet (Bronstein et al., 1996). A simplified explanation of balance control will be given below in connection to Figure 2.1. Excursions of the CoM may result from an external disturbance (e.g. force by a push) or may have an internal cause such as noise in one of the sensory systems; here represented by the proprioceptive, visual and vestibular system. Furthermore, there may be irregularities in the muscle activities (motor noise). The sensory signals are integrated and processed by the central nervous system and are then used to command and perform muscular activity (Neuromuscular Controller), producing a corrective joint torque that counteracts the CoM excursion. The corrective torque leads to ground reaction forces, which can be measured using a force plate under the feet in the form of a Centre of Pressure (COP) shift. CoM excursion and joint torques are mutually related to each other. 


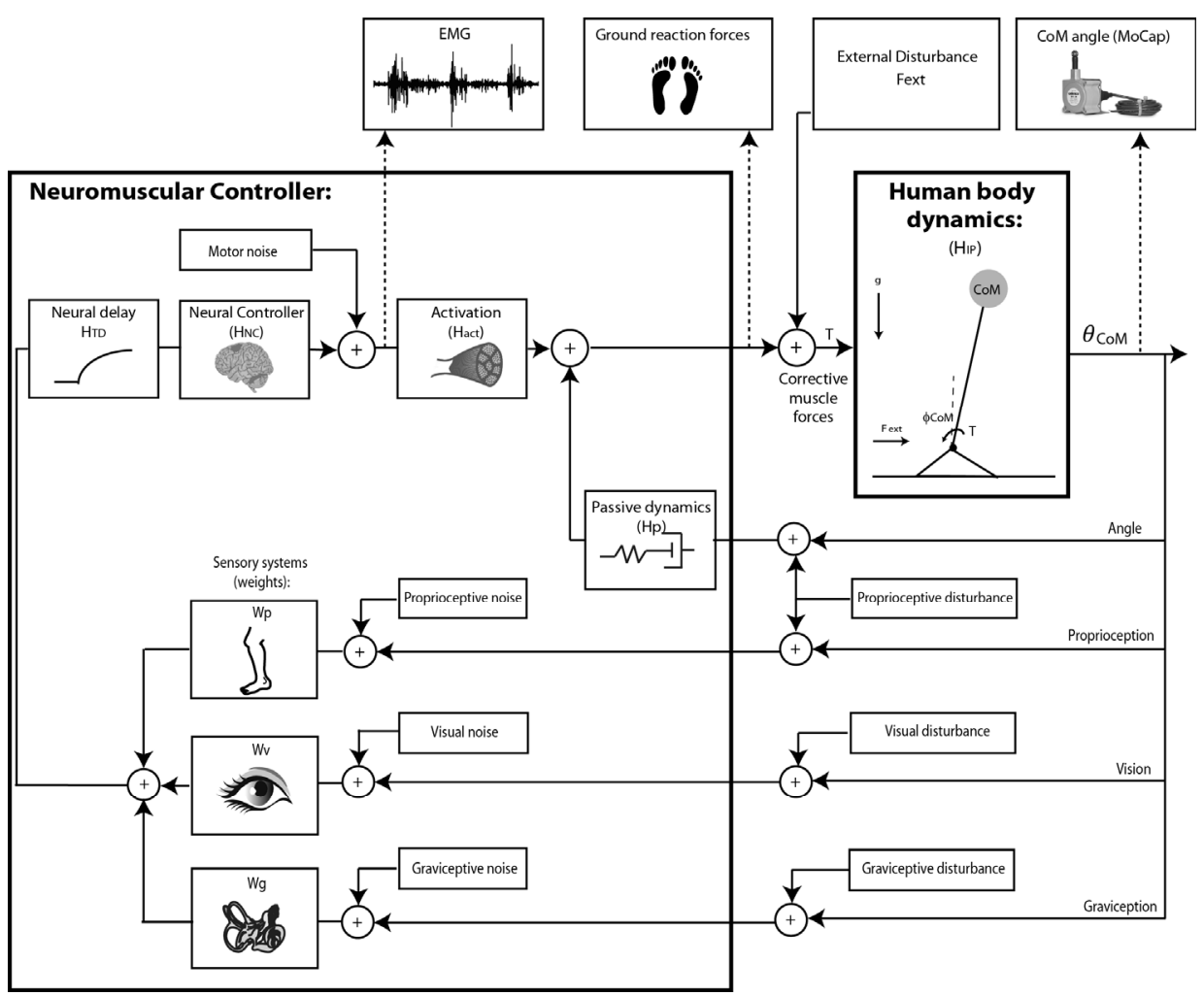

Figure 2.1: A basic model of balance control, based on (van der Kooij et al., 2005): The human body is described by the dynamics of an inverted pendulum $\left(H_{I P}\right)$ with the sum of the corrective joint torque $(T)$ and an external disturbance force $\left(F_{\text {ext }}\right)$ as input and the body movement around the ankle $\left(\theta_{C O M}\right)$ as output. The ankle movement is sensed by the sensory systems; proprioceptive $\left(W_{p}\right)$, visual $\left(W_{v}\right)$ and vestibular $\left(W_{g}\right)$. Each system has addition of unknown sensory noise in the feedback loop. Based on the reliability of the sensory information, the sensory weights are changed and the integrated information is sent with a lumped neural time delay $\left(H_{T D}\right)$ to a neural controller $\left(H_{N C}\right)$. Both active muscle activation dynamics $\left(H_{a c t}\right)$ and passive muscle dynamics $\left(H_{p}\right)$ determine the corrective force and torque to maintain standing balance. Balance control can be disturbed by external mechanical disturbances and experimentally disturbing each of the sensory systems, e.g. by moving the visual scene. Balance control can then use different strategies to maintain standing balance, e.g.by putting less weight on the disturbed sensory system. The dotted lines indicate which signals can be measured in balance control studies with appropriate equipment: motion capturing systems for CoM excursions, EMG for muscle activity, and force plates for the ground reaction forces. 
This system is called in the terminology of control engineers a closed-loop feedback mechanism (Peterka, 2003; van der Kooij et al., 2005). If one investigates standing balance and ignores this closed-loop feedback mechanism, causes and effects of impaired balance will be hard to determine (Laessoe et al., 2007; Mancini and Horak, 2010; Nutt et al., 2011) and identification of the deteriorated underlying system is almost impossible. A recent review (Visser et al., 2008) shows that the existing clinical balance tests are unable to reveal how well the underlying systems are functioning and have little or no influence on the choice of the therapy of individual patients. It is to mention, however, that assessment of standing balance across different studies is currently limited due to differences in subject selection criteria, data collection methods, and analysis and interpretation of the results. Also the therapeutic aims should be defined. Assessment of standing balance should aim at an early detection of the cause of impaired standing balance, i.e. identification of the primarily deteriorated underlying system, and of the compensation strategies that are at work and should be the desired goal of therapy (van der Kooij et al., 2005; Mancini and Horak, 2010). Tailored interventions and prevention strategies to reduce the consequences of impaired balance in the elderly, will be cost effective.

In this paper we will expand an overview of important current tests that assess standing balance, to novel techniques that are able to unravel the closed-loop feedback mechanism and to identify causes and effects, i.e. the underlying system deteriorations and the adopted compensation strategies. This can be the basis for developing interventions that specifically address impaired standing balance and thereby reduce falls (Kingma et al., 2011).

\subsection{Balance assessment methods}

For assessing standing balance, three major approaches can be distinguished: Clinical balance tests, posturography, and Closed Loop System Identification Techniques (CLSIT).

\section{Clinical balance tests}

In the most widely used clinical balance tests, the clinician scores the ability of the subject to perform a motor task in which balance control is involved. For example, the Short Physical Performance Battery (SPPB) (Guralnik et al., 1994) consist of three types of physical activities: balance tests, gait speed tests, and the chair stand test. The Berg Balance Scale (BBS) (Berg et al., 1989) is a set of simple balance related tasks, ranging from rising from a chair to standing on one foot. The timed Up and Go test (TUG) (Mathias et al., 1986; Podsiadlo and Richardson, 1991) determines the time that a subject takes to rise from a chair, walk three meters, turn around, walk back to the chair and sit down. Although these clinical balance tests all assess a subject's balance, each test focuses on a different aspect of balance control.

The test-retest reliability of these clinical balance tests is high ( $>80 \%)$. However, the tests differ in specificity and sensitivity, because of ambiguous outcome measures; either assessment of recurrent falls (over different time periods), prediction of future falls or identification of impaired standing balance. Even within one test, conflicting specificities and 
sensitivities are found, due to variations in the population of interest and differences in physical activity between the populations (Visser et al., 2008; Mancini and Horak, 2010; Panzer et al., 2011; Merlo et al., 2012). Clinical balance tests often relate to fall history, but it is hard to determine whether a person is at risk of future falls, especially if this person has not fallen before (Pickering et al., 2007). Another drawback of the clinical balance tests is that falls in elderly are very multifactorial and do not solely depend on the ability to perform certain tasks that involve balance. The important questions - which underlying system is primarily affected and which compensation strategies are at work - are not addressed by clinical balance tests, making it next to impossible to give targeted therapy on an individual level.

\section{Posturography}

In posturography, inertial sensors and a force plate have replaced the subjective eye of the clinician in the clinical balance tests. Measuring the CoM excursions and CoP shifts aim to quantify the quality of standing balance. The general assumption is that abnormal CoM excursions stem mainly from internal problems within the closed-loop feedback mechanism and abnormalities in the CoP shifts reflect more the adequacy of the subject's strategy to maintain standing balance (Visser et al., 2008).

In static posturography, subjects are asked to maintain standing balance during quiet stance. Dynamic posturography uses experimentally induced balance disturbances to determine the resilience against these disturbances. When a specific type of disturbance (e.g. standing on a tilting support surface) results in an abnormally large CoM excursion or CoP shift, this may indicate some deterioration in the underlying systems of balance control (Bronstein et al., 1996; Visser et al., 2008; Mancini and Horak, 2010). The Sensory Organization Test (SOT) is used to identify deterioration in an individual sensory system (Anon, 2011). However, comparing the SOT in specificity and sensitivity to clinical balance tests is hard, as the goal of the SOT is to identify the quality of the sensory systems and not to assess the cause of recurrent falls or to predict future risk of falling (Panzer et al., 2011).

Interpretation of CoM excursions and CoP shifts remains difficult, as less or more CoM or CoP movement is not simply good or bad. For example, muscle activities and joint torques can compensate for CoM excursions, but can also initiate CoM excursions (Laughton et al., 2003). Therefore, enlarged CoM or CoP movements can indicate deterioration of an underlying systems as well as a proper strategy to maintain standing balance; cause and effect relations remain unclear. A recent study has shown that deterioration in the motor system in terms of reduced muscle mass does not necessarily affects the CoP shifts associated with balancing (Bijlsma et al., 2013).

In summary, all tests are per se ambiguous and fail to reveal latent deteriorations in balance control, as there exist various many compensation strategies in and across the underlying systems. The outcomes of the currently used tests are unable to reveal how well the underlying systems are functioning and they have little or no influence on the choice of the therapy of individual patients (Visser et al., 2008). 


\section{Closed loop system identification techniques}

A new engineering method termed Closed Loop System Identification Technique (CLSIT), is aimed to detect the functioning of the underlying systems, the deterioration - even if it is compensated for - together with the influence of internal disturbances on standing balance. To explain CLSIT, balance control is compared to a box. For the bare eye it is possible to determine the size of the box (i.e. the ability to maintain standing balance as determined by clinical balance tests). By picking up the box, the weight of the box can be determined (i.e. the quality of standing balance as determined by posturography). However, the mechanisms working inside the box remain unclear. CLSIT reveals the quality and contribution of underlying systems and the used balance strategies by sending well defined external disturbances into the box at one end and measure the reactions on the other end; i.e. CoP and CoM movements and corrective joint torques. This is often called a 'black box' approach or, when additional knowledge is included (e.g. about sensors, neural or motor properties from physiology), a 'grey box' approach. These approaches allow to distinguish between the causes (e.g. a deteriorated sensory system) and the effects (e.g. higher muscle activation) of impaired standing balance in the closed-loop balance control system (van der Kooij et al., 2005).

\subsection{Method of closed loop system identification}

CLSIT originates from system and control theory and describes standing balance in the form of a simplified model where the human body and a neuromuscular controller are schematically shown (Figure 2.1). The simplest representation of the human body is an inverted pendulum. The body mass is concentrated in the CoM that balances on lumped legs, with a pivot point at the ankle joint. The neuromuscular controller contains all the mechanisms and properties that are required to keep the CoM within the base of support and includes the sensory systems, the nervous system and the motor system (van der Kooij et al., 2005).

\section{Dynamics of the neuromuscular controller}

Using CLSIT, the goal is often to describe the behaviour of the neuromuscular controller, i.e. how balance is regulated over time and under specific test conditions. The neuromuscular controller receives information about the CoM excursions and responds by generating muscle forces that provide the corrective torques in the joints. As mentioned earlier, various underlying systems interact within this neuromuscular controller, allowing humans to use various balance strategies. CLSIT aims to distinguish between: (1) The contribution of each leg in generating corrective muscle forces to keep the CoM within the base of support (van Asseldonk et al., 2006) (2) The contribution from various joints - such as the ankle and the hip - in generating these forces (Boonstra et al., 2013); (3) The separate contribution from intrinsic properties of the muscle-skeletal system (passive control) and the neural components (active control) in the generation of the forces (de Vlugt et al., 2002, 2006; Ludvig et al., 2011); (4) The sensitivity of the sensory systems - proprioception, vision and vestibular - and their individual contribution to neuromuscular control (Peterka, 2002; 


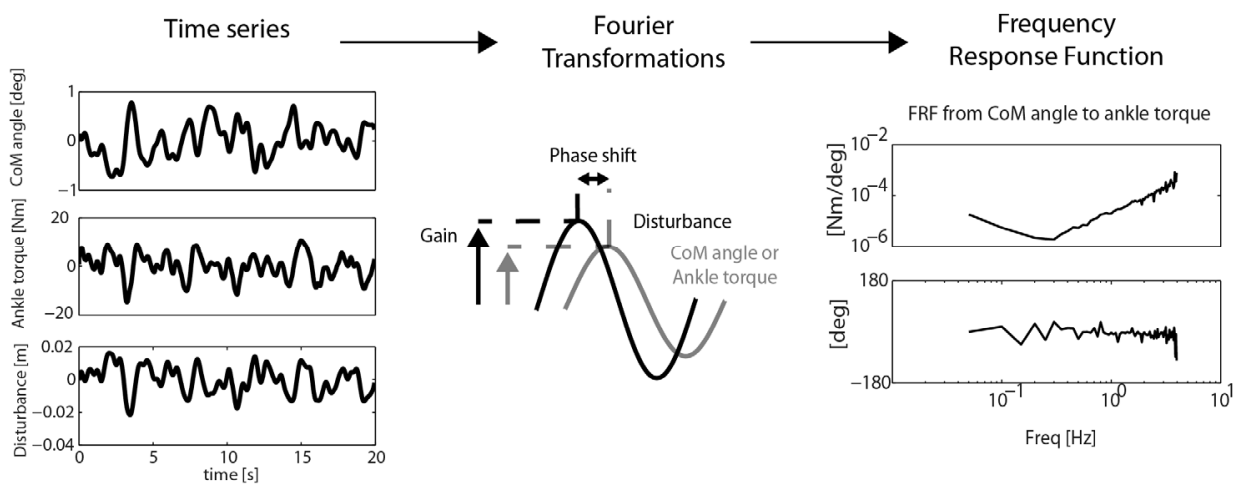

Figure 2.2: Closed Loop System Identification Technique: measured time series are decomposed in cycles (repetitions of the disturbance signal) and transformed to the frequency domain by means of Fourier Transformation. Both COM angle and corrective ankle torque are related to the disturbance by determining the magnitude and phase for all frequencies, which results in the Frequency Response Function of the neuromuscular controller.

Doumas and Krampe, 2010; Jeka et al., 2010; Pasma et al., 2012); (5) The effect of sensory and motor noise on the reliability of the received information and the accuracy of the generated muscle forces (van der Kooij and Peterka, 2011). To study all the effects within the neuromuscular controller, CLSIT use specifically designed and externally applied disturbances; either mechanical (support surface motion or pushes having impact on the human body) or sensory (providing or abandoning inputs to the sensory systems) (Fitzpatrick et al., 1996; van der Kooij et al., 2005; van Asseldonk et al., 2006; Kiemel et al., 2008, 2011).

\section{External Disturbances and Frequency Response Functions}

The externally applied disturbances are specifically designed as humans respond differently to either fast (high frequency) or slow (low frequency) disturbances. Therefore the external disturbances in CLSIT include a wide range of frequencies in order to capture the human responses in one measurement that may last several minutes (van der Kooij et al., 2005). Furthermore, the disturbance signals are unpredictable as humans typically adjust to predictable disturbances and their responses may habituate (Keshner et al., 1987; Bloem et al., 1998). All disturbances are submaximal to measure standing balance and prevent intentional loss of standing balance and falls.

Humans can either suppress or enhance disturbances and respond directly or with a certain delay. The differences between the response and the disturbance in means of amplitude and time can be displayed - termed Frequency Response Function (FRF) - which consists of two parts, a magnitude and a phase curve, respectively (Mergner et al., 2005). Figure 2.2 shows an example of a FRF estimation where a disturbance (support surface translation) evokes a body lean (COM angle), which the neuromuscular controller counteracts by producing ankle torque. Note that for higher frequencies the magnitude increases, meaning 
more corrective ankle torque is produced - compared to lower frequencies - to compensate for the body lean. The phase is slightly negative for higher frequencies, meaning the response lags behind the perturbation; a reaction takes place instead of anticipation to the disturbance. These FRFs can be compared between or within subjects to track changes in balance control across different mechanical and sensory stimulus conditions. However, an additional step is needed to translate the FRFs to physiologically interpretable parameters, termed parameter estimation.

\section{Parameter estimation}

The above described FRF shows the behaviour of the system, but does not reveal which physiological mechanisms are underlying the system. Therefore, each subsystem of standing balance control (Figure 2.1) can be described by a mathematical formula with parameters describing the physics. It is then possible to use this formula in a computational model, and to compare the model outcomes with experimental data from humans, which may give physiological meanings to the outcomes. These parameter values can be compared within and between a group of healthy young subjects and a group of elderly.

\subsection{Application of system identification}

In summary, CLSIT is required to unravel cause and effects by applying specific external disturbances to study the dynamic behaviour of the neuromuscular controller. By parameter estimation one can quantify the contents in the black-box of balance control. Information can be drawn from application of either mechanical disturbances or sensory disturbances. Mechanical disturbances are especially helpful in estimating the underlying balance strategies of the neuromuscular controller. Sensory disturbances allow estimating the quality of the sensory systems and their contribution to the neuromuscular control.

\section{Mechanical disturbances}

Mechanical disturbances are often aimed to identify deteriorations in the nervous system part of the control and in the strategies used. Figure 2.1 shows the simplest representation of the human body biomechanics, which is a single inverted pendulum with lumped legs pivoting about the ankle joints (which actually reflects moderate body sway in the sagittal plane quite well). However, humans have two legs and it has been shown that it is possible to identify the contribution of each leg in generating muscle forces, depending on the weight distribution (van Asseldonk et al., 2006). This is done by estimating the FRFs of the left and right leg, i.e. the contribution of each leg to standing balance. In healthy humans, the weight distribution between the legs directly corresponds to the relative contribution of each leg to standing balance. However, asymmetries are recognized in patients with hemispheric stroke (van Asseldonk et al., 2006) and Parkinson's Disease (van der Kooij et al., 2007; Geurts et al., 2011). These patients sometimes bear most of the body weight on one leg, although the other leg contributes most to balance control. Balance asymmetry is difficult to detect with the bare clinical eye or to quantify with traditional posturography (van der Kooij et al., 2007) as these tests do not measure the differences between the legs in 
maintaining standing balance. Furthermore, with mechanical disturbances the quality of inter-segmental coordination can be assessed, i.e. the contributions of several joints to standing balance (Boonstra et al., 2013). Humans use their ankle joints to maintain standing balance, but may also involve the hips, especially when disturbances are large or fast (Horak and Nashner, 1986). The body biomechanics is then represented in the model by a double inverted pendulum with a leg segment and a Head-Arms-Trunk segment. To be able to use the CLSIT method, two independent mechanical disturbances are needed, one for each segment (Boonstra et al., 2013) (for example a support-surface movement and a force at the hip level). The FRF description of the neuromuscular controller is now expanded to four terms. There are two direct terms, covering the FRFs from ankle angle to ankle torque and from hip angle to hip torque. These direct terms quantify the ankle and hip contributions to balance control. Furthermore, there are two indirect terms, which cover the FRFs from ankle angle to hip torque and from hip angle to ankle torque and reflect the inter-segmental couplings (Boonstra et al., 2013). Furthermore, mechanical disturbances can be applied to identify motor system impairments. By using the CLSIT method, the separate contribution of the intrinsic muscle properties (passive control) and the neural components (active control) in the generation of muscle force can be studied (Kiemel et al., 2008, 2011).

\section{Sensory Disturbances}

Sensory disturbances are used to quantify the visual, proprioceptive and vestibular contributions to maintain standing balance. The relative contribution of each sensory system in a given balancing task can be expressed by sensory weighting factors in the model. Humans are able to change these weights, for example either using a specific sensory system more (up-weighting) or less (down-weighting) (Peterka, 2002).

Visual information can be disturbed by visual scene rotations. It has been found that subjects effectively adapt to scene rotation by down-weighting the disturbed visual information and up-weighting information from the proprioception and the vestibular organ (Peterka, 2002; Goodworth and Peterka, 2012). Vestibular loss patients appear to depend foremost on visual and proprioceptive information and are less able to re-weight their sensory information (Peterka, 2002). They use other strategies to maintain standing balance, which cannot prevent, however, that they are more prone to falls in case the proprioceptive or visual information is unreliable or conflicting. Support surface rotation around the ankle axis has been used to specifically evoke proprioceptive responses (Schouten et al., 2011; Pasma et al., 2012). Using such an approach for each leg individually, asymmetries in sensory reweighting between the legs can be detected (Pasma et al., 2012). The vestibular system can be disturbed selectively by galvanic stimulation of the labyrinths, which allows studying sensory reweighting of the vestibular input with respect to vision or proprioceptive information (Cenciarini and Peterka, 2006). Finally, the CLSIT method allows to estimate the effect of sensory and motor noise on the reliability of the obtained human data (van der Kooij and de Vlugt, 2007; van der Kooij and Peterka, 2011). 


\subsection{Conclusion on clinical relevance}

Early detection of causal relations in the pathogenesis of impaired standing balance as is possible with CLSIT allows for timely diagnosis and tailored therapy. CLSIT has therefore a high potential to improve (cost) effectiveness in geriatric care. Bringing new techniques like CLSIT under the attention of clinicians and future care planners allows for acceptance and evaluation of their potential without unnecessary delays. Firstly, CLSIT is able to detect deteriorations in the neural system. It has been shown that abnormal multi-segmental postural reflex patterns and inter-limb couplings may contribute to falls (Marigold and Eng, 2006). Secondly, CLSIT is able to identify sensory deteriorations. For example, sensory receptors may degrade and thereby become severely affected by noise, which makes the measurement of sensory functions in elderly and patient populations a relevant issue (Cohen et al., 1996). Finally, CLSIT is able to estimate deteriorations in the motor system using parameter estimation, and it allows estimating the relative contribution of the intrinsic muscle properties to the generation of muscle force in comparison to the active contribution through reflexes.

Currently, the ultimate goal in assessing balance is to detect elderly who are at risk of falls, which then allows for timely and targeted therapeutic interventions and/or rehabilitation. At present, fall history is still the only and best predictor of future falls (Ganz et al., 2007). In order to bring the CLSIT method into clinics, it is necessary to prove clinical effectiveness. This includes sensitivity and specificity analysis between a healthy young population, a healthy elderly population, and an elderly population with a known deterioration of a specific underlying system, in which the long-term outcome ends in falling. Additionally the testretest ability of CLSIT must be determined and compared to existing clinical balance tests. Evaluation of cost-effectiveness and long term benefits for the patient in terms of quality of life should be an inherent part of the evaluation process.

Future investigations also have to consider the effect of adaption in standing balance, which so far in this paper was not considered in relation to the CLSIT method. It is known that humans constantly tend to adapt to changing environments and tasks. If such adaptations occur abnormally slow, subjects are more likely to fall (Doumas and Krampe, 2010). Future studies that use the CLSIT method must be expanded to cover this important aspect of standing balance. The CLSIT method then will represent a powerful clinical tool to detect causal relations for the identification of impaired standing balance at an early stage. This is the base for designing prevention strategies and the development of targeted interventions to reduce the consequences of deteriorated balance in the elderly population. Cost effectiveness of measures to prevent falls and reducing and/or delaying nursing home admissions is obvious but remains an important aspect of evaluation of the merits of introducing new approaches in healthcare. 


\title{
Chapter 3
}

\section{Comparison of closed loop system identification methods to quantify multi-joint human balance control}

D. Engelhart, T.A. Boonstra, R.G.K.M. Aarts, A.C. Schouten and H. van der Kooij. Submitted

\begin{abstract}
The incidence of impaired balance control and falls increases with age and disease and has a significant impact on daily life. Detection of early-stage balance impairments is difficult as many intermingled mechanisms contribute to balance control. Current clinical balance tests are unable to quantify these underlying mechanisms, and it is therefore difficult to provide targeted interventions to prevent falling. System identification techniques in combination with external disturbances may provide a way to detect impairments of the underlying mechanisms. This is especially challenging when studying multi-joint coordination, i.e. the contribution of both the ankles and hips to balance control. With model simulations we compared various existing non-parametric and parametric system identification techniques in combination with external disturbances and evaluated their performance. All methods are considered multi-segmental (both the ankles and the hips contribute to maintaining balance) closed-loop balance control. Validation of the techniques was based on the prediction of time series and frequency domain data. Parametric system identification could not be applied in a straightforward manner in human balance control due to assumed model structure and biological noise in the system. Although the time series were estimated reliably, the dynamics in the frequency domain were not correctly estimated. Nonparametric system identification techniques did estimate the underlying dynamics of balance control reliably in both time and frequency domain. The choice of the external disturbance signal is a trade-off between frequency resolution and measurement time and thus depends on the specific research question. With this overview of the applicability as well as the (dis)advantages of the various system identification techniques, we can work toward the application of system identification techniques in a clinical setting.
\end{abstract}




\subsection{Introduction}

Maintaining a stable upright posture is a complex task. The body is inherently unstable due to the gravitational pull, and it would fall without stabilizing control. The central nervous system (CNS) stabilizes the body by integrating sensory feedback signals to determine the appropriate response, which is sent to the muscles and results in corrective joint torques to keep the body upright (Peterka, 2003). How the stabilizing mechanism of the CNS regulates balance can be investigated by estimating the dynamics of the so-called neuromuscular controller that outputs corrective joint torques as a response to body sway (Engelhart et al., 2014).

With aging or due to a disease, sensory systems or the neuromuscular controller can deteriorate and as a result, balance control problems can arise (Pasma et al., 2014b). For example, the elderly often have difficulties maintaining balance during daily life activities, and this impaired balance is a strong risk factor for falls (Rubenstein, 2006; Muir et al., 2010). About 28-35 percent of people aged over 65, fall each year and this incidence increases with age (WHO, 2007). To determine who is at risk of falling, clinicians use clinical balance tests (e.g. Berg Balance Scale (Berg et al., 1989)) and posturography measures (e.g., sensory organization test (Cohen et al., 1996)). These tests assess the ability to maintain standing balance and the quality of balance by measuring the body sway. However, these tests do not determine the contribution and quality of the underlying mechanisms (Engelhart et al., 2014; Pasma et al., 2014b). In addition, it is currently not possible to determine who has an increased risk of falling in the next year (Ganz et al., 2007; Laessoe et al., 2007; Visser et al., 2008). Therefore, it is difficult to provide targeted interventions to decrease fall incidence. In other words, there is a clear (clinical) need to be able to a) identify people with an increased fall risk, b) evaluate targeted interventions, and c) improve our overall understanding of the pathophysiology of balance-control impairments (Visser et al., 2008; Kingma et al., 2011; Sibley et al., 2013).

Estimation of the neuromuscular controller dynamics is difficult. As in a closed-loop feedback system (such as balance control) it is hard to disentangle cause and effect. That is, without externally applied disturbances, it is difficult to determine if, for example, changes in muscle activity result in changes in muscle force that will affect body sway, or that the opposite is true, i.e. changes in body sway are detected by sensors and transmitted to the nervous system that excites the muscle groups reflected in changes in the muscles' electromyography (EMG). Furthermore, standing balance is regulated around the ankles and hips, and multi-joint coordination must be provided. Movements of one segment influence movements of the other segment (Horak and Nashner, 1986; Park et al., 2004), resulting in additional interactions.

System identification techniques in combination with specifically designed external disturbances provide a way to disentangle cause and effect in balance control. Therefore, our group (van der Kooij et al., 2005, 2007; van Asseldonk et al., 2006; Boonstra et al., 2014b; Engelhart et al., 2014; Pasma et al., 2014b) and other groups (Johansson et al., 
2001; Peterka, 2002; Kim et al., 2009, 2012; Jeka et al., 2010; Mergner, 2010; Goodworth and Peterka, 2012) have developed and evaluated novel quantitative balance-control assessment methods based on system identification techniques to better understand the balance-control system, with the ultimate goal to improve clinical decision making. As the balance control system is dynamic (i.e. its response is described as a function of time), system identification techniques can be used to determine the underlying structures of the system by unravelling cause-and-effect relations in multi-joint coordination. The field of system identification is very broad, with many approaches and various techniques and methods. It is far from trivial to compare the methods reported in literature, as there are always differences in experimental design and the results are presented in different ways. If meaningful interpretation and comparisons are to be derived from balance-control experiments in different labs, there is a clear need for standardized protocols (Visser et al., 2008).

In this paper we compare different multivariable system identification techniques, as applied in literature, and evaluate the effects of various disturbance types and analysis methods, using model simulations. The advantage of model simulations is that all methods were validated based on one system from which all dynamics are known. We focused on methods that approached the human balance-control system as a double-inverted pendulum, pivoting at the ankles and hips in the anterior-posterior direction. This is contrary to many other methods that have approached the balance-control system as an inverted pendulum, with only an ankle joint. Our approach was chosen because recent studies have shown that differences between e.g. Parkinson's disease patients and the elderly (Boonstra et al., 2014a) and between the elderly and young (Accornero et al., 1997; Hsu et al., 2013) were the most pronounced in multi-segmental balance-control coordination.

Here, we give an overview of the applicability and (dis)advantages of various system identification techniques, which will aid toward the use of standardized measurement protocols to access balance control with system identification techniques in a clinical setting.

\subsection{Methods}

This section describes the general goal of system identification in human balance control, i.e. estimating the dynamics of the neuromuscular controller. By simulating a two-segmental balance-control model that contains the dynamics of the underlying physiology, various system identification techniques were presented, validated, and compared.

\section{Modelling of human balance control}

Figure 3.1 shows a model of human balance control, in which the underlying physiology is described by various underlying mechanisms. When only considering anterior-posterior movement, the body dynamics can be regarded as a double-inverted pendulum, consisting of two segments; the lumped legs and the head-arms-trunk (hat) segment pivot around the ankle and hip joint respectively. Internal disturbances (biological noise in muscles, sensory organs, and the nervous system) and external disturbances (pushes and pulls on the human 
body and the pull of gravity) drive the system away from equilibrium. Maintaining standing balance is a feedback system, i.e. a closed-loop system. The sensory systems (visual, vestibular, and proprioceptive) give information about the body position and velocity relative to the environment. These signals are processed and integrated by the CNS and fed back (with a neural transport delay) to the muscles. Corrective joint torques result from the activation of muscles (reflexive dynamics), together with intrinsic properties of the muscleskeletal system (intrinsic dynamics). The entire neuromuscular controller describes how balance is regulated and is the system of interest in this study. This neuromuscular controller has separate feedback paths for the leg and hat segment and is therefore a multiple-input-multiple-output (MIMO) system.

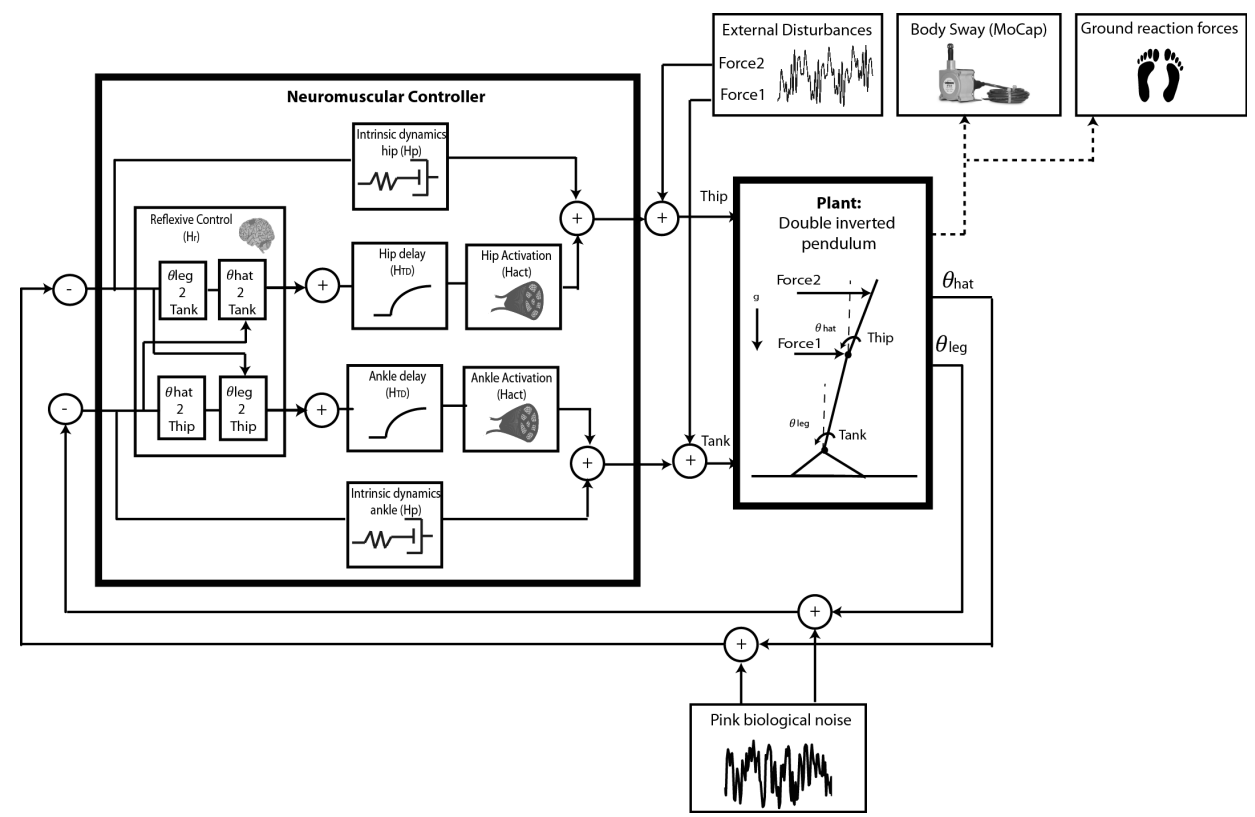

Figure 3.1: Simple representation of the human balance control system. The human body is represented by a double inverted pendulum, with a leg and a hat-arms-trunk (hat) segment. The neuromuscular controller generates corrective joint torques $\left(T_{\text {ank }}, T_{\text {hip }}\right)$ to regulate balance over time, by intrinsic dynamics $\left(H_{p}\right)$ together with time delayed $\left(H_{T D}\right)$ reflexive activation $\left(H_{r}, H_{a c t}\right)$ of muscles. For system identification purposes the body is disturbed by two external force disturbances at the hip and shoulder level, and body sway and ground reaction forces are measured. 


\section{System identification approach}

\section{External disturbances}

Describing the dynamics of a (MIMO) system requires knowledge of the inputs and outputs of the system of interest. Here, the inputs of the neuromuscular controller are the leg and hat segment angles and the outputs are the corrective ankle and hip joint torques. In addition, an independent disturbance for each degree of freedom in the system is required (Pintelon and Schoukens, 2012). In this study we are interested in the contribution of the ankles and hip joints in balance control and therefore two disturbances must be applied. Applying only one disturbance can give erroneous results (Boonstra et al., 2013), as responses remain intertwined. There exist various choices where to apply the two external disturbances (Fujisawa et al., 2005; Kiemel et al., 2011; Boonstra et al., 2013), as long as they generate sufficient responses around each degree of freedom in the system. In this study, continuous push and pull disturbances were applied at the level of the hip and between the shoulder blades (Engelhart et al., in press), see Figure 3.1.

The external disturbances for system identification purposes are specifically designed as humans respond differently to fast or slow disturbances. The dynamic behaviour of interest typically extents from $0.01 \mathrm{~Hz}$ to frequencies up until 4 to $5 \mathrm{~Hz}$ (van der Kooij and de Vlugt, 2007). Therefore, the external disturbances are broadband and excite the system in the region of interest. Furthermore, humans typically adjust to predictable disturbances and their responses may habituate. Therefore, the disturbances are unpredictable. Finally, the disturbances are submaximal. Therefore general stance behaviour is identified without making people step or fall. Various designs of such disturbance signals are described in the next paragraph.

\section{Frequency Response Functions}

All system identification techniques presented here assume linear and time-invariant (LTI) human behaviour. This implies that the system does not change over the course of the experiment and that participants do not change strategy (e.g. switch from responding stiff to slack); i.e. the behaviour does not change over time. Furthermore, when the participant is disturbed with a periodic signal, he/she will show periodic responses and the responses scale proportionally with the disturbances.

In such a LTI system, the human responses contain the same frequencies as the disturbances and the behaviour of the system can be described in the frequency domain by a Frequency Response Function (FRF). The FRF consists of complex numbers, which can be expressed in a magnitude and phase. The entire dynamic behaviour is displayed with a magnitude and phase plot that describes the differences in amplitude and timing respectively, between the inputs and outputs of a system as a function of frequency $(f)$.

To be more specific, the FRF of the neuromuscular controller $\left(H_{c}(f)\right)$ describes the dynamic relation, in the frequency domain, between the corrective joint torques $\left(T_{a n k}, T_{\text {hip }}\right)$ due to deviations in the segment angles $\left(\theta_{\text {leg }}, \theta_{\text {hat }}\right)$ and consists of four terms: 


$$
\left[\begin{array}{c}
T_{\text {ank }}(f) \\
T_{\text {hip }}(f)
\end{array}\right]=\left[\begin{array}{ll}
H_{c, \theta_{\text {leg } 2 T a n k}}(f) & H_{c, \theta_{\text {hat }} 2 T_{\text {ank }}}(f) \\
H_{c, \theta_{\text {leg }} 2 T_{\text {hip }}}(f) & H_{c, \theta_{\text {hat }} 2 T_{\text {hip }}}(f)
\end{array}\right]\left[\begin{array}{l}
\theta_{\text {leg }}(f) \\
\theta_{\text {hat }}(f)
\end{array}\right]
$$

There are two direct terms covering the FRFs from leg angle to ankle torque $\left(H_{c, \theta_{l e g} 2 T_{\text {ank }}}\right)$ and from hat angle to hip torque $\left(H_{c, \theta_{\text {hat }} 2 T_{\text {hip }}}\right)$. Furthermore, there are two indirect terms, which cover the FRFs from leg angle to hip torque $\left(H_{c, \theta_{l e g} 2 T_{h i p}}\right)$ and from hat angle to ankle torque $\left(H_{c, \theta_{\text {hat }} 2 T_{\text {ank }}}\right)$, which reflect the inter-segmental coupling between the segments (Boonstra et al., 2013).

\section{Disturbance signals}

There are various design options for external disturbance signals. For system identification purposes, the disturbance signal should be sufficiently rich (power at many frequencies) and unpredictable to prevent a contribution of anticipation to the postural response. For proper application in the MIMO case, all the disturbance signals must be independent. Previous model simulations showed that disturbance signals with a flat power spectrum gave the best results, i.e. the lowest signal to noise ratio over the frequency range of interest (Boonstra et al., 2013).

Here, we focus on two main categories of disturbance signals, multisine and filtered noise. These two signals were commonly used in balance-control experiments where mechanical disturbances were applied (Engelhart et al., in press; de Vlugt et al., 2006; Kiemel et al., 2011; Boonstra et al., 2014a). To make a fair comparison between the multisine and filtered noise signals, the frequency content of both signals was comparable. Furthermore, the root mean square (RMS) of the disturbances was the same to ensure equal energy was added to the system. Finally the amount of data was kept comparable between the signals (aiming for a measurement time of three minutes). Various designs of multisine and filtered noise signals were used in the simulations, as described below and shown in Figure 3.2.

\section{Multisine}

Multisine signals consist of a sum of sinusoids, hence, the signals only contain power at specific frequencies. When each harmonic fits exactly an integer number of times in the multisine signal, leakage in the frequency domain analysis can be prevented. This is assured by only including harmonics with frequencies equal to an integer multiple of the frequency resolution. As the frequency resolution is the inverse of the period of the multisine signal, the lowest frequency of interest is therefore directly related to the measurement time. To apply multisine signals for MIMO application, two multisine signals can have equal frequency content, but in this case the signals are not independent. To create the independent signals for each input, the experiment is repeated with two different combinations of the multisine (Pintelon and Schoukens 2001).

In this study, a random phase multisine signal with a period of 20 seconds was generated, in which a total of 27 frequencies were logarithmically spaced in a frequency band of 0.05 and $5 \mathrm{~Hz}$. Here, the multisine signals were repeated five times, resulting in a trial length of 
100 seconds. In the first trial, both inputs were excited with the same multisine, while in the second trial the sign of the second input was changed. This resulted in a total measurement time of 200 seconds, approximately three minutes.

\section{Zippered Multisine}

Another possibility is to design two multisine signals with one excited frequency only appearing in one signal. The input signals are chosen such that each disturbance contains a number of excited frequencies at an interleaved frequency grid, termed a zippered multisine (Pintelon and Schoukens, 2012). The advantage compared to a multisine disturbance is that a zippered multisine fully characterizes a MIMO system from a single experiment. To characterize a closed-loop system with zippered multisine requires interpolation to the full frequency grid, which is described in the data analysis section.

In this study, a zippered multisine disturbance was generated in which a total of 27 frequencies were logarithmically spaced in a band between 0.05 and $5 \mathrm{~Hz}$. From each set of three odd harmonics, one was randomly assigned to the first disturbance, one was randomly assigned to the second disturbance, and the third one was not excited. The zippered multisine had a period of $20 \mathrm{~s}$ and were repeated nine times, leading to a measurement time of $180 \mathrm{~s}$.

\section{Filtered noise}

White noise consists of all frequencies with equal power and is a representation of a purely stochastic process. As each value is uncorrelated to the other values, two white noise disturbances are naturally independent.

In this study, two filtered noise signals of 180 seconds were generated comparable to (Kiemel et al., 2011). Two white noise signals were passed through a first-order high-pass filter with a cut-off frequency of $0.05 \mathrm{~Hz}$ and an eight-order Butterworth low-pass filter with a cut-off frequency of $5 \mathrm{~Hz}$. The power spectral density was computed using Welch's method with a 20-second (comparable to the length of the multisine signal) Hanning window and 50 percent overlap. This created a flat power spectrum comparable to the multisine signals. The filtered noise signals had a length of 180 seconds.

\section{Cyclic filtered noise}

It is also possible to generate cyclic filtered noise as input signals to allow for averaging in time over the sequences to reduce noise. Two filtered noises of 20 seconds were generated, similar as described above. The signals were repeated nine times, leading to a measurement time of $180 \mathrm{~s}$. 

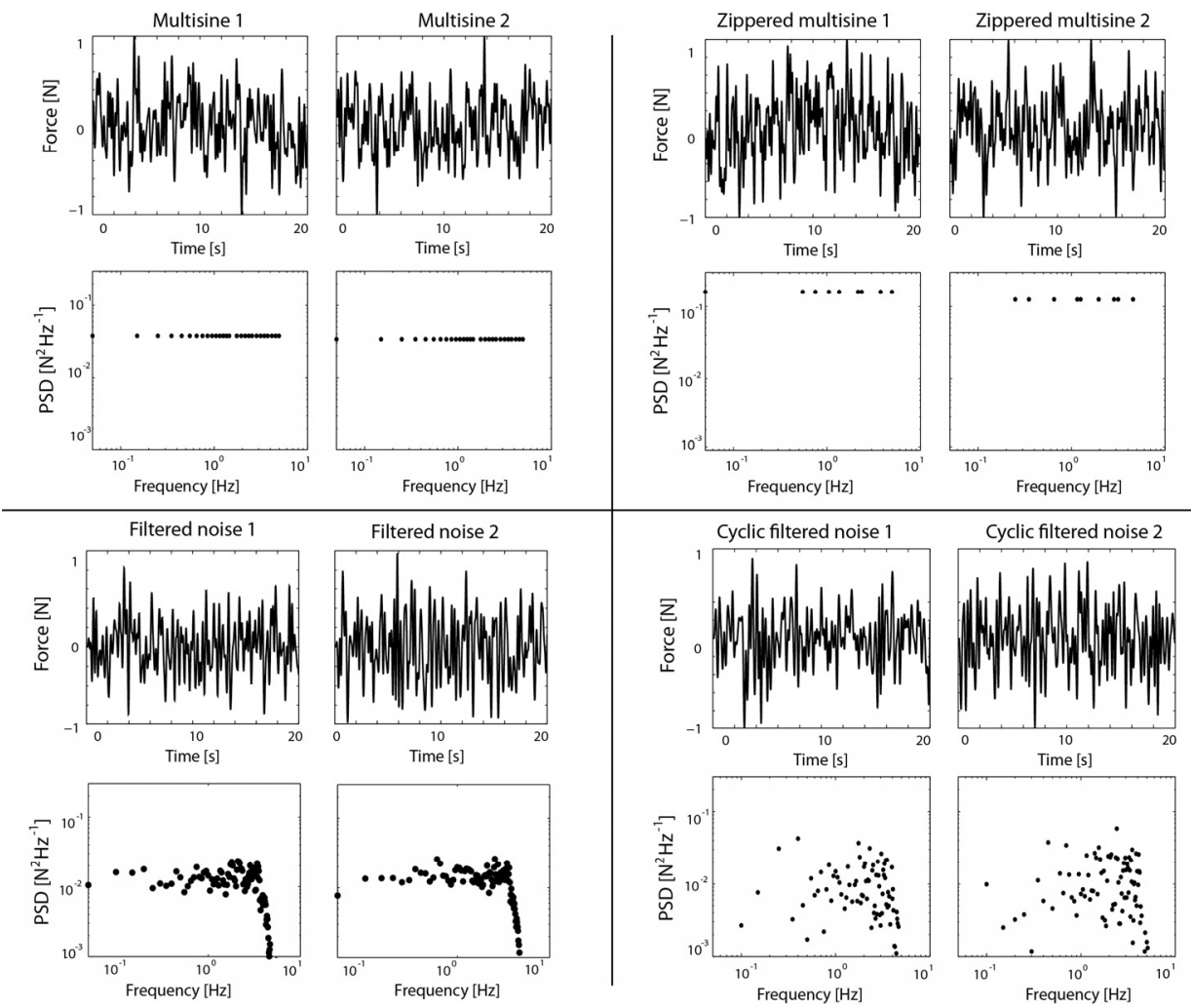

Figure 3.2: Various disturbance signals for MIMO system identification. In the top rows the time series and in the bottom rows the power spectral densities (PSD). The filtered noise has a length of 180 seconds, but for comparison with the other signals, only 20 seconds are shown. The PSD of the filtered noise is smoothened by welch averaging, with a 20 seconds Hanning window, with $50 \%$ overlap. 


\section{Model simulations}

Model simulations were performed on the two-segment human balance control model. (Matlab Simulink (The MathWorks, Natick, MA)) with a leg and hat segment, and a neuromuscular controller as shown in Figure 3.1. The model was based on a previous study (Boonstra et al., 2013) and is extensively described in the Appendix. Two external force disturbances with a peak-to-peak amplitude of $80 \mathrm{~N}$ were applied at the level of the hip and the shoulders in five simulated trials of 180 seconds, in which the disturbance signals varied:

1a) Two multisine disturbances with equal frequency content.

1b) Disturbance signals as in trial 1 , in which the sign of one perturbation is reversed

2) Zippered multisine disturbance

3) Filtered noise disturbance

4) Cyclic filtered noise disturbance

These simulations rendered the time series of the segment angles and joint torques at a sample frequency of $1 \mathrm{kHz}$, which were processed in Matlab (The MathWorks, Natick, MA). To make the model simulations realistic and to compare them to experimental measurements, additional noise was included. Biological noise originates from inaccuracies in the sensory systems and the motor system. This biological noise is believed to be of pink origin, meaning the power spectral density (power per frequency $(f)$ ) is inversely proportional to the frequency $\left(\frac{1}{f^{a}}\right)$. In our simulations, pink noise with $a=1.2$, was added to the system, such that during quiet stance the sway angle (remnant sway) was comparable to data of standing balance (van der Kooij and Peterka, 2011). Furthermore, measuring human responses, includes measurement noise due to the equipment. This was modelled as a zero mean white noise source (variance of $1 * 10^{-6}$ ) added to the states (segment angles and angular velocities), and to the ankle and hip joint torques.

Table 3.1: Various system identification techniques with corresponding disturbance signals. Some methods perform well with a specific disturbance signal (indicated by a $\sqrt{ }$ ), as the performance with another disturbance signal is poor (indicated by $\mathrm{a} x$ )

\begin{tabular}{lcc}
\hline & Multisine & Filtered nois \\
\hline Non-parametric system identification techniques & & $\mathrm{x}$ \\
1) Two experiments (2EXP) & $\sqrt{ }$ & $\mathrm{x}$ \\
2) Interpolation (INT) & $\sqrt{ }$ & $\sqrt{ }$ \\
3) Partial coherence (PC) & $\mathrm{x}$ & $\sqrt{ }$ \\
Parametric system identification techniques & & $\sqrt{ }$ \\
4) Multiple least square (MLSQ) & $\sqrt{ }$ & $\sqrt{ }$ \\
5) Optimization (OPTIM) & $\sqrt{ }$ & $\sqrt{ }$ \\
6) Autoregressive-moving-average model with & & $\sqrt{ }$ \\
exogenous inputs (ARMAX) & &
\end{tabular}




\section{Data analysis}

Based on the applied disturbances and the rendered segment angles and joint torques, various system identification techniques were used to analyze the simulated data and estimate the dynamic behaviour of the neuromuscular controller. The methods are divided in two main categories: non-parametric system identification techniques and parametric system identification techniques (Table 3.1). Please note that the use of methods and disturbance signals is intertwined. Also note that although the model is simulated in continuous time, the system identification techniques are based on sampled data and therefore the analysis is time discrete. The performance of the methods was evaluated based on validation criteria in the time and frequency domain, as described at the end of this section.

\section{Non parametric system identification techniques}

Non-parametric system identification techniques express the system behaviour in the frequency domain by means of an FRF, without a priori knowledge of the system. To extract the neuromuscular controller properties from the closed loop system, the Joint-InputOutput-Method is applied (van der Kooij et al., 2005). The closed-loop is opened by relating the signals in the loop to the external disturbances. The FRF of the neuromuscular controller $\left(H_{c}(f)\right)$ is described by:

$$
H_{c}(f)=-S_{d T}(f)\left(S_{d \theta}(f)\right)^{-1}
$$

In which $S_{d T}$ and $S_{d \varphi}$ are the cross-spectral density (CSD) matrices between the external disturbances $(d(f))$ to the segment angles $(\theta(f))$ and joint torques $(T(f))$. The methods as described below, differ in the applied external disturbance signal and thereby the method of calculating the CSD matrices.

Method 1: Two experiments (2EXP)

This method is based on previous studies (Pintelon and Schoukens, 2012; Boonstra et al., 2013, 2014b), where the external disturbance signals are multisine disturbances. The disturbance signals were applied in two experiments, in which the sign of the disturbance is reversed in the second experiment, compared to the first experiment.

The time series of the disturbance signals, segment angles and joint torques $(d(t), \theta(t), T(t))$ were segmented in 10 repetitive cycles of the disturbance signal (two experiments with five cycles). The segmented time series were averaged to reduce noise, prior to analysis. Subsequently, data was transformed to the frequency domain by a fast Fourier transformation $(d(f), \theta(f), T(f))$ and the CSD matrices from the external disturbances to the segment angles $\left(S_{d \theta}(f)\right)$ and joint torques $\left(S_{d T}(f)\right)$ were calculated. The FRF of the neuromuscular controller $\left(H_{c}(f)\right)$ resulted from equation (3.2).

\section{Method 2: Interpolation (INT)}

This method is based on a previous studies (Pintelon and Schoukens, 2012; Engelhart et al., 2014), where the external disturbance signals are zippered multisine. The time series of the disturbance signals, segment angles and joint torques $(d(t), \theta(t), T(t))$ were segmented in 
nine repetitive cycles of the disturbance signal. Subsequently, data was transformed to the frequency domain by a fast Fourier transformation. The Fourier coefficients of the disturbances, angles and torques $(d(f), \theta(f), T(f))$ were averaged in the frequency domain to reduce noise, prior to analysis. Calculating the CSD matrices $\left.\left(S_{d \theta}(f)\right), S_{d T}(f)\right)$, requires that the matrix components are known at all excited frequencies, which is untrue for the zippered multisine, as both input signals contain different frequencies. Therefore, the complex numbers of the cross spectral densities were interpolated in terms of magnitude and phase.

Consequently, all matrix components for the full range of excited frequencies in the zippered multisine were obtained and it was possible to identify the neuromuscular controller $\left(H_{c}(f)\right)$ using equation 3.2.

An additional advantage of using multisine, is that the assumption of linearity can be tested. As only random odd harmonics are excited, even nonlinearities become visible when there is power at the even frequencies, while the odd non-excited frequencies can be used to detect and quantify the power level of the odd nonlinearities. For model simulations, the outcomes are trivial (as the equations of motion are linearized), but for experiments one can obtain additional information about the reliability of the FRF.

\section{Method 3: Partial Coherence (PC)}

This method is based on previous studies (Perreault et al., 1999; de Vlugt et al., 2003, 2006; Kiemel et al., 2011), where the external disturbance signals are filtered noise. The time series of the disturbance signals, segment angles, and joint torques $(d(t), \theta(t), T(t))$ were transformed to the frequency domain by a fast Fourier transformation $(d(f), \theta(f), T(f))$. Because both filtered noise disturbances contain equal frequency content (matrix division is possible) and they are naturally uncorrelated, elicited responses can be related to either disturbance. The CSD matrices $\left.\left(S_{d \theta}(f)\right), S_{d T}(f)\right)$ were computed using Welch's method with $20 \mathrm{~s}$ Hanning windows and 50 percent overlap among nine frequency bands. Consequently, the FRF of the neuromuscular controller $\left(H_{c}(f)\right)$ was calculated with equation 3.2.

\section{Parametric system identification techniques}

Parametric system identification techniques express the behaviour of a system in a model with a limited number of parameters. The behaviour of a system is described by a mathematical function, relating signals with respect to time. Often a priori assumptions about the order of the system and the model structure are required. Method 4 and 5 as described below only estimate the dynamic behaviour of the system, while methods 6 and 7 also give an estimation of the noise in the system. 
Method 4: Multiple Least Squares (MLSQ)

Early studies (Barin, 1989; Winter et al., 2001) used a multiple least squares algorithm to compute a feedback gain matrix $(K)$, which describes the relation between the states $(x(t))$ of the human balance control system and the corrective joint torques $(T(t))$ in the time domain.

$$
\begin{gathered}
T(t)=-K x(t) \\
{\left[\begin{array}{c}
T_{\text {ank }}(t) \\
T_{\text {hip }}(t)
\end{array}\right]=-\left[\begin{array}{llll}
k_{11} & k_{12} & d_{11} & d_{12} \\
k_{21} & k_{22} & d_{21} & d_{22}
\end{array}\right]\left[\begin{array}{c}
\theta_{\text {leg }}(t) \\
\theta_{\text {hat }}(t) \\
\dot{\theta}_{\text {leg }}(t) \\
\dot{\theta}_{\text {hat }}(t)
\end{array}\right]}
\end{gathered}
$$

The feedback gain matrix $\mathrm{K}$ was found by a least square estimation:

$$
K=-\mathbb{T} \mathbb{X}^{T}\left(\mathbb{X X}^{T}\right)^{-1}
$$

where $\mathbb{X}$ and $\mathbb{T}$ are matrices in which each row corresponds to a state or a corrective joint torque, respectively, and each column corresponds to a time sample. The feedback gain matrix $\mathrm{K}$ was used to determine the FRF of the neuromuscular controller; $H_{c}(f)$ is quantified as a combination of stiffness $(k)$ and damping $(d)$ values

$$
\begin{gathered}
{\left[\begin{array}{l}
T_{\text {ank }}(f) \\
T_{\text {hip }}(f)
\end{array}\right]=-H_{c}\left[\begin{array}{l}
\theta_{\text {leg }}(f) \\
\theta_{\text {hat }}(f)
\end{array}\right]} \\
H_{c}=\left[\begin{array}{ll}
k_{11}+d_{11} * j 2 \pi f & k_{12}+d_{12} * j 2 \pi f \\
k_{21}+d_{21} * j 2 \pi f & k_{22}+d_{22} * j 2 \pi f
\end{array}\right]
\end{gathered}
$$

In which $j 2 \pi f$ expresses the relation between the angles and angular velocities in the frequency domain.

In this study, we applied both multisine as well as filtered noise disturbances, to excite the system. The time series of the states, i.e. segment angles and angular velocities $(\theta(t), \dot{\theta}(t))$ and the joint torques $(T(t))$ were segmented in nine repetitive cycles of the disturbance signal. The segmented time series were averaged to reduce noise, prior to analysis.

Method 5: Optimization (OPTIM)

In a more recent study (Park et al., 2004), an optimization algorithm was used for estimating the feedback gain matrix $\mathrm{K}$. Equation 3.6 shows that different from the multiple least squares method, the states $(x(t)$, segment angles, and angular velocities) were simulated (in continuous time) based on knowledge of the biomechanics of the human (assumed to be a double-inverted pendulum (2IP) and captured in an $A_{2 I P}$ and $B_{2 I P}$ matrix), the estimated $K$ matrix, and the applied disturbances (matrix $W$ ). Consequently, the corrective joint torques were derived from the states and the estimated $K$ matrix. The optimal feedback gain matrix $K$ was based on the lowest error between the estimated and true states and torques. 


$$
\begin{array}{ll} 
& \dot{x}(t)=A_{2 I P} x(t)+B_{2 I P} u(t)+W \\
& u(t)=T(t)=-K x(t) \\
& \dot{x}(t)=\left(A_{2 I P}-B_{2 I P} K\right) x(t)+W
\end{array}
$$

In this study, we applied both multisine as well as filtered noise disturbances to excite the system. The time series of the states, i.e. segment angles and angular velocities $(\theta(t), \dot{\theta}(t))$ and joint torques $(T(t))$ were segmented in nine repetitive cycles of the disturbance signal. The segmented time series were averaged to reduce noise, prior to analysis. The optimization algorithm was repeated 10 times using initial guesses (random values within bounds) for $K$, to check for local minima in the optimization. If a minimum was found repeatedly, a global minimum was assumed and the corresponding $K$ matrix was implemented in equation 3.5 to estimate the neuromuscular controller $H_{c}(f)$.

Method 6: Autoregressive-moving-average model with exogenous inputs (ARMAX)

Another commonly used group of parametric system identification techniques are prediction error methods (PEM). In PEM methods, the output of the system as obtained from measurements is compared to the output as predicted by the model. The difference between the two is termed the prediction error and indicates how well the model represents the data. The model includes parameters that describe the system behaviour, and additionally includes parameters that describe the noise in the system. Various model structures have different parameterization of the system and noise model (Ljung, 1999). In a previous study (Fujisawa et al., 2005), an ARMAX model was used to model balance control, in which output $y(t)$ is related to the input $u(t)$ and noise $e(t)$ :

$$
A\left(z^{-1}\right) y(t)=B\left(z^{-1}\right) u(t)+C\left(z^{-1}\right) e(t)
$$

In which $z^{-1}$ is the shift operator to describe past discrete time samples. The $\mathrm{A}$ and $\mathrm{B}$ polynomial matrices describe the estimation of the system, how $u$ (the segment angles) are related to $y$ (the joint torques), depending on a number of parameters (order of the system). Increasing the number of parameters will improve estimation, as the estimated structure better compares to the data. However, a large number of parameters increases the computational burden and possibly does not further reduce the prediction error. A tradeoff between the lowest number of parameters and a low prediction error must be made, which is often expressed in the Akaike's Information Criterion (Ljung, 1999).

The validity of the estimation (consistency) can be determined by residuals analysis. The residuals are the remaining errors between the real model and the identified model. When the autocorrelation of the residuals represents white noise, this is an indication that the noise dynamics are fully captured. The cross-correlation between the inputs and residuals indicates whether or not the system model is captured. An advantage of these residual tests is that the accuracy of a model is determined by only using the available data. Residual outcomes directly explain whether the identified model can represent the system dynamics within specified confidence levels. 
In this study, we applied both multisine and filtered noise disturbances. As we are dealing with a MIMO and closed-loop system, first a consistent ARMAX model was estimated for the relation between the disturbances and torques, resulting in $H_{d T}(f)$. Subsequently a consistent ARMAX model was estimated for the relation between the disturbances and angles, resulting in $H_{d \theta}(f)$. The obtained models are divided resulting in an estimation of the neuromuscular controller properties, comparable to equation 3.2.

Method 7: Predictor Based Subspace Identification (PBSID)

In the predictor based subspace identification method (Ljung, 1999) (van Wingerden, 2008), the LTI system is considered in state space:

$$
\begin{gathered}
x\left(t+t_{s}\right)=A_{s s} x(t)+B_{s s} u(t)+K_{s s} e(t) \\
y(t)=C_{s s} x(t)+D_{s s} u(t)+e(t)
\end{gathered}
$$

with a sample time $t_{s}$ and where $x(t), u(t), y(t)$ are the state, input and output vectors as functions of time, respectively. The vector $e(t)$ denotes the zero mean white noise sources. The state space matrices $A_{s s}, B_{s s}, C_{s s}, D_{s s}, K_{s s}$ are the system, input, output, direct feedthrough, and observer matrices. The goal of the PBSID method is: Given the input sequence $u(t)$ (the two disturbances) and output sequence $y(t)$ (ankle and hip angles and corrective torques), find all the state space matrices of the system up to a global similarity transformation. If the states $x(t)$ were known, the solution would be straight forward: compute $\mathrm{C}$ and $\mathrm{D}$ with linear regression, reconstruct the noise $e(t)$, and compute $A_{s s}, B_{s s}, K_{s s}$ with linear regression. However, the problem is to find the states. An essential step in subspace identification is to reconstruct the (extended) observability matrix $(\Gamma)$ from input and output data (Verhaegen, 2007).

$$
\Gamma^{n}=\left[\begin{array}{c}
C_{s s} \\
C_{s S} A_{s s} \\
\vdots \\
C_{S S} A_{s S}{ }^{n-1}
\end{array}\right]
$$

A system is said to be observable if the states of the system can be inferred through linear combinations of the system outputs. This means that from knowledge of the system's outputs it is possible to determine the behaviour of the entire system. The system is observable when the rank of this matrix equals the amount of states $(n)$, which gives the order of the system. A singular value decomposition can be used for order determination. Once the rank and the observability matrix are known, the $A_{s s}$ and $C_{s s}$ matrix can be determined as matrix $C_{s s}$ equals the first rows (dependent on the number of outputs) of the observability matrix and $A_{s s}$ can be determined from the rest. Additionally, the states and the noise contributions can be estimated, together with the $B_{S S}$ and $D_{S S}$ matrices with linear regression.

Due to the state-space description of the system, the method can implicitly handle MIMO systems, and common structures between disturbances, segment angles and corrective torques are incorporated. Also as advantage comparable to PEM methods, determination of the system order was incorporated in the algorithm and no a priori assumptions about the 40 
model structure were needed. The estimates for both $H_{d T}(f)$ and $H_{d \theta}(f)$ were therefore found in just one identification step, with the disturbances expressed as two inputs and the segment angles and corrective joint torques as four outputs.

$$
\left[\begin{array}{c}
\theta_{\text {leg }}(f) \\
\theta_{\text {hat }}(f) \\
T_{\text {ank }}(f) \\
T_{\text {hip }}(f)
\end{array}\right]=\left[\begin{array}{l}
H_{d \theta}(f) \\
H_{d T}(f)
\end{array}\right]\left[\begin{array}{c}
d_{\text {pelvis }}(f) \\
d_{\text {shoulder }}(f)
\end{array}\right]
$$

Based on the transfer function matrices for both $H_{d T}$ and $H_{d \theta}$, the neuromuscular controller can be obtained using equation 3.2. In this study, we applied both multisine as well as filtered noise disturbances.

\section{Validation Criteria}

To quantify how well the obtained neuromuscular controller descriptions represent the true system, we have used validation criteria for both the time and frequency domain.

\section{Goodness of fit (GOF)}

Based on simulated data, the theoretical FRF of the neuromuscular controller can be compared with the identified FRF of the system identification method. This goodness of fit (GOF) is determined by an approximation of the integral of the object value (OV) via the trapezoidal method:

$$
G O F=\frac{1}{n_{f}} \sum_{f=0.05}^{4} \frac{O V(f)+O V(f+1)}{2} \Delta f
$$

For each frequency in the disturbance signal the OV is calculates as the logarithmic absolute and squared difference between the estimated neuromuscular controller FRF $\left(\widehat{H_{c}}(f)\right)$ and the theoretical $\operatorname{FRF}\left(H_{c}(f)\right)$.

$$
O V=\left|\ln \left(H_{c}(f)\right)-\ln \left(\widehat{H}_{c}(f)\right)\right|^{2}
$$

A low GOF indicates a good fit. As the disturbance signals contain a different amount of frequencies (i.e., multisine $n_{f}=27$, zippered multisine $n_{f}=18$, filtered noise $n_{f}=100$ ) the GOF is normalized with respect to the number of frequencies.

\section{Variance Accounted For (VAF)}

Once a parametric estimate of the system is available, the behaviour of the neuromuscular controller can be used to predict the outputs of the system by simulating the model using the corresponding inputs. The percentage of Variance Accounted For (VAF) represents the quality of the identified model by comparing the measured output $(y(t))$ with the simulated output $(\hat{y}(t))$ of the identified model (6) in the time domain. The VAF of two equal signals will be 100 percent. If they differ, the VAF will be lower (Verhaegen, 2007). 


$$
\operatorname{VAF}(y(t), \hat{y}(t))=\left(1-\frac{\operatorname{var}(y(t)-\hat{y}(t))}{\operatorname{var}(y(t))}\right) * 100 \%
$$

In case of a non-parametric estimate, the Fourier components of the open loop transfer functions $S_{d T}(f)$ and $S_{d \theta}(f)$ were multiplied with the Fourier components of the disturbances. The inverse Fourier transform of this product, results in the outputs of the system; the angles or torques respectively. Comparing these 'simulated' outputs to the measured outputs gives the VAF. In case of cyclic disturbances (multisine and zippered multisine signals), this measure describes how each cycle deviates from the mean over cycles, which is comparable to a Noise-to-Signal-Ratio.

\section{Measurement time}

In balance control experiments it is advantageous to have short experimental measurement times. Especially in impaired standing balance, subjects become easily fatigued, which induces time variant behaviour. Therefore the amount of measurement time, i.e. amount of data needed for identification is also a validation factor and was studied by using various simulation times.

\subsection{Results}

\section{Non parametric system identification techniques}

Non-parametric spectral-system identification techniques were especially designed for the estimation of system dynamics in the frequency domain. Figure 3.3 shows that with nonparametric system- identification techniques, the neuromuscular controller dynamics were estimated well. Hence, for all methods and various external disturbance signals, the estimated dynamics were close to the theoretical FRF.

Table 3.2 gives an overview of the validation criteria for the various methods. In the frequency domain, the fits were all good, as can be seen from the relatively low GOF values. The GOF value for the 2EXP method was lowest, followed by the PC method, and the INT method had the highest GOF value. In the time domain, the VAF values were high for all methods. The 2EXP and INT method had a slightly higher VAF than the PC method.

The amount of measurement time was kept similar between the non-parametric system identification techniques. Exciting the system with multisine signals had the advantage that in case this periodic signal was repeated during the experiment (which we did), averaging over the successive repetitions decreased the noise. This potentially reduced the amount of data that was needed to obtain a reliable non-parametric estimate of the neuromuscular controller. Increasing the amount of repetitive cycles of a multisine signal decreased the GOF, i.e. the FRF resembled the theoretical one better. The GOF did not decrease with increased measurement time for the zippered multisine, possibly due to the interpolation of the zippered multisine which induces and error. Averaging over more cyclic filtered noise repetitions decreased noise in the system and estimated the FRF better; however the effect was less than for the multisine. Segmenting a filtered noise signal in more segments and 
apply smoothing using a Hanning window to compute the CSD also reduced noise and gave a better estimation. However, the drawback was that it also influenced the lowest frequency, which you could identify.

\section{Parametric system identification techniques}

Parametric-system identification techniques were especially designed for the estimation of system dynamics in the time domain. Figure 3.4 shows the simulated and identified time series for all the parametric methods. The identified time series were comparable to the simulated time series, indicating a good fit. Table 3.2 shows the VAF values were all high $(>85 \%)$, indicating a good estimation of the system dynamics in the time domain. The VAF values for the filtered noise disturbances were slightly higher compared to the application of multisine disturbances.

However, when the estimated model was evaluated in the frequency domain, the methods performed less, as can be seen from the relatively high GOF values. The estimated dynamics deviated from the theoretical FRF of the neuromuscular controller. Figure 3.4 clearly shows the mismatch between the theoretical and estimated FRF.

Comparable to the non-parametric system identification techniques, the amount of measurement time was kept similar between the parametric system identification techniques. Again, applying a periodic signal (such as a multisine) improved the estimation of the mean responses as noise was averaged out. The feedback gains or estimated model were therefore a more reliable representation of the system. However, in parametric models, the estimated model depended much more on the model structure and the order than on the amount of available data. 


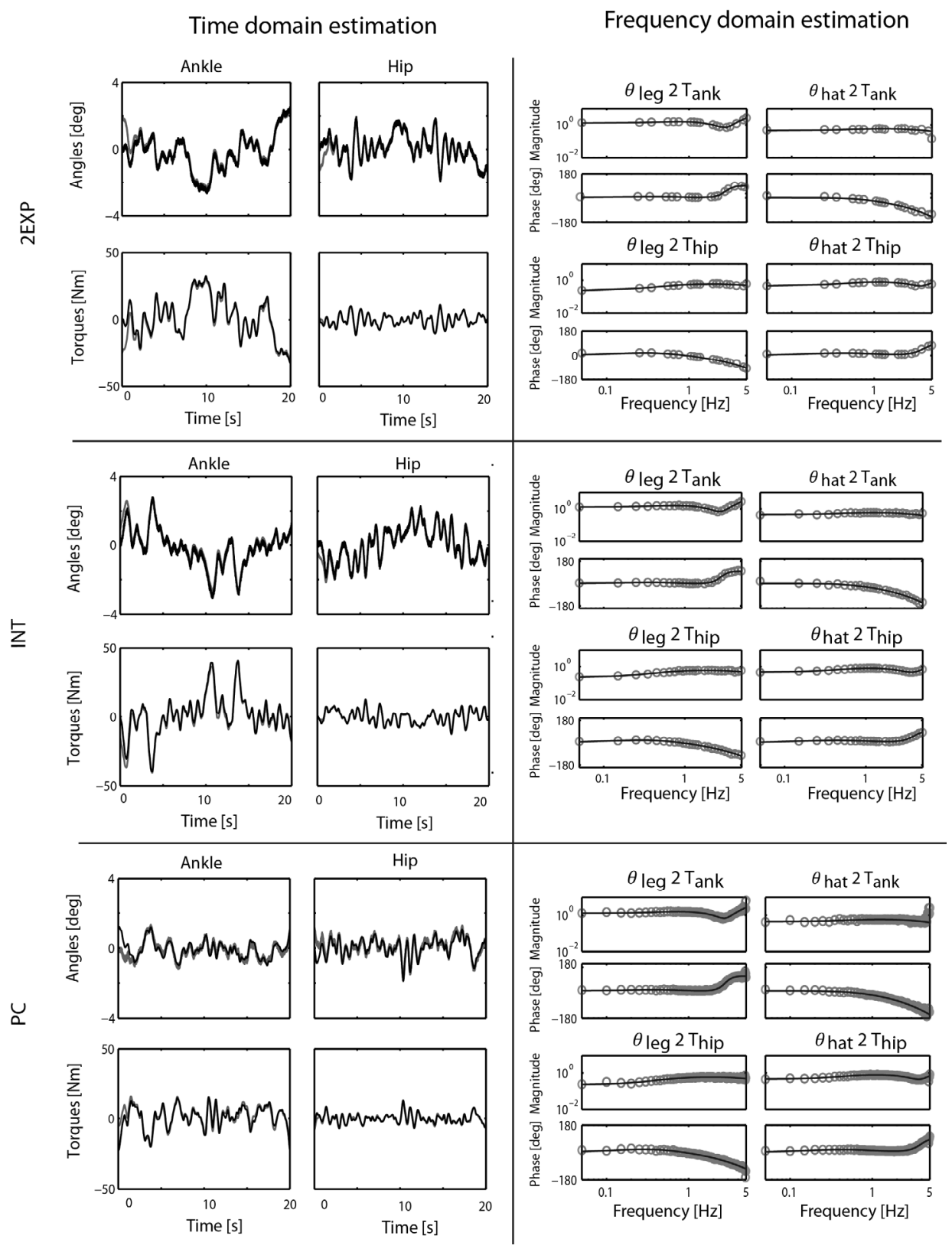

Figure 3.3: Non parametric system identification of the neuromuscular controller in time domain (left) and frequency domain (right) based on simulated data. For the time domain estimation, the simulated time series are shown in black and the estimated time series are shown in grey. For the frequency domain estimation, the theoretical FRF of the model is shown by the black solid line and the estimated FRF on the excited frequencies is shown by the grey circles. 


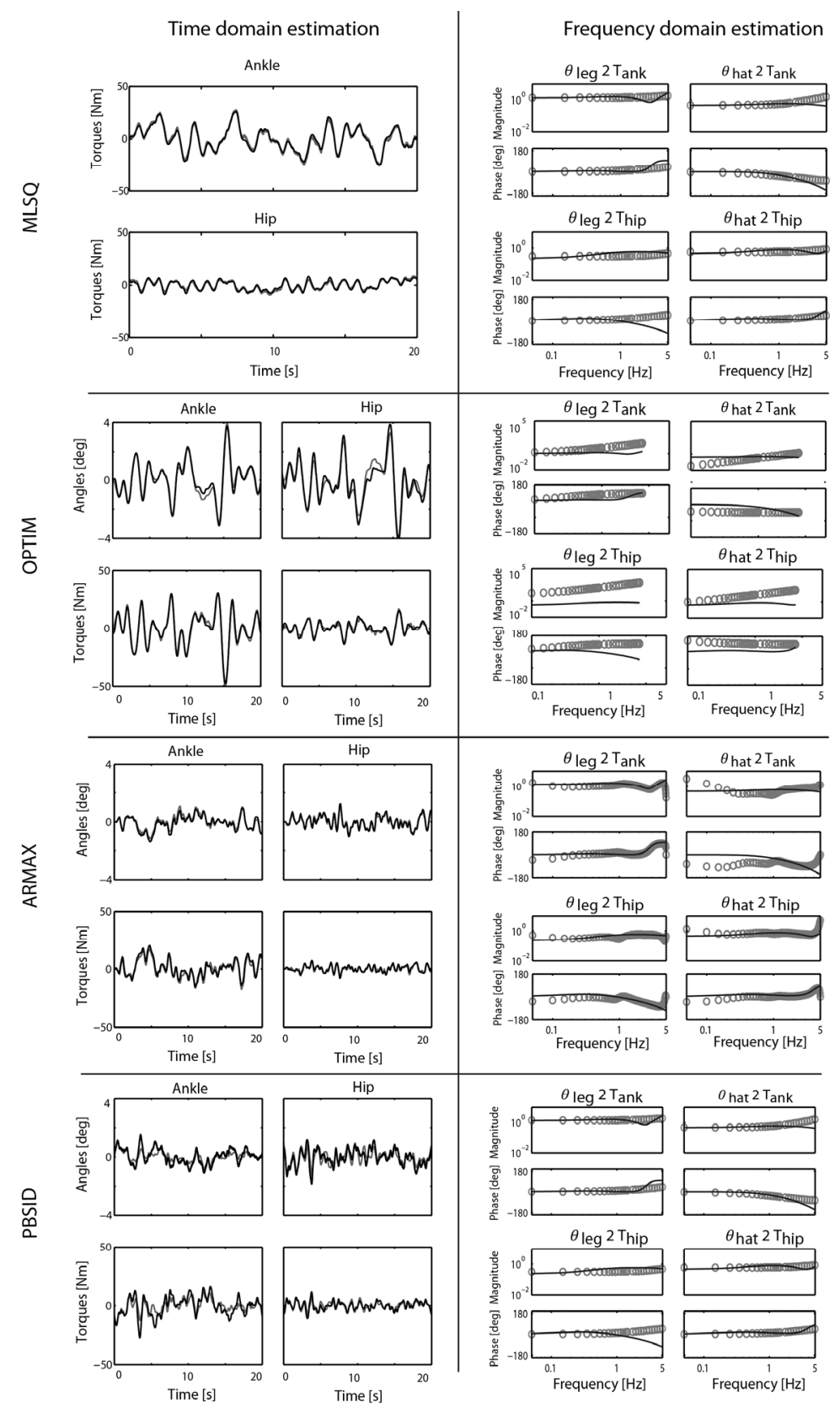

Figure 3.4: Parametric system identification of the neuromuscular controller in time domain (left) and frequency domain (right) based on simulated data. For the MLSQ and OPTIM, multisine signals were used and for the ARMAX and PBSID method, filtered noise signals were used. For the time domain estimation, the simulated time series are shown in black and the estimated time series are shown in grey. For the frequency domain estimation, the theoretical FRF of the model is shown by the black solid line and the estimated FRF on the excited frequencies is shown by the grey circles. 
Table 3.2: Validation of the system identification techniques, based on model simulations

\begin{tabular}{|c|c|c|c|c|c|c|c|c|}
\hline & \multicolumn{8}{|c|}{ Multisine } \\
\hline & \multicolumn{4}{|c|}{ GOF } & \multicolumn{4}{|c|}{ VAF [\%] } \\
\hline & $\theta_{\text {leg }} 2 T_{\text {ank }}$ & $\theta_{\text {hat }} 2 T_{\text {ank }}$ & $\theta_{\text {leg }} 2 T_{\text {hip }}$ & $\theta_{\text {hat }} 2 T_{\text {hip }}$ & $\theta_{\text {leg }}$ & $\theta_{\text {hat }}$ & $T_{a n k}$ & $T_{\text {hip }}$ \\
\hline 2EXP & 0.07 & 0.23 & 0.09 & 0.03 & 97 & 97 & 98 & 99 \\
\hline INT & 4.64 & 4.09 & 2.73 & 1.54 & 98 & 99 & 99 & 99 \\
\hline PC & - & - & - & - & - & - & - & - \\
\hline MLSQ & 2.81 & 4.81 & 13.34 & 0.84 & - & - & 98 & 92 \\
\hline OPTIM & 8.56 & 53.85 & 138.82 & 63.49 & 94 & 90 & 96 & 88 \\
\hline ARMAX & 6.48 & 18.32 & 11.06 & 6.39 & 94 & 98 & 93 & 85 \\
\hline PBSID & 2.37 & 2.23 & 10.24 & 0.51 & 0 & 0 & 0 & 0 \\
\hline
\end{tabular}

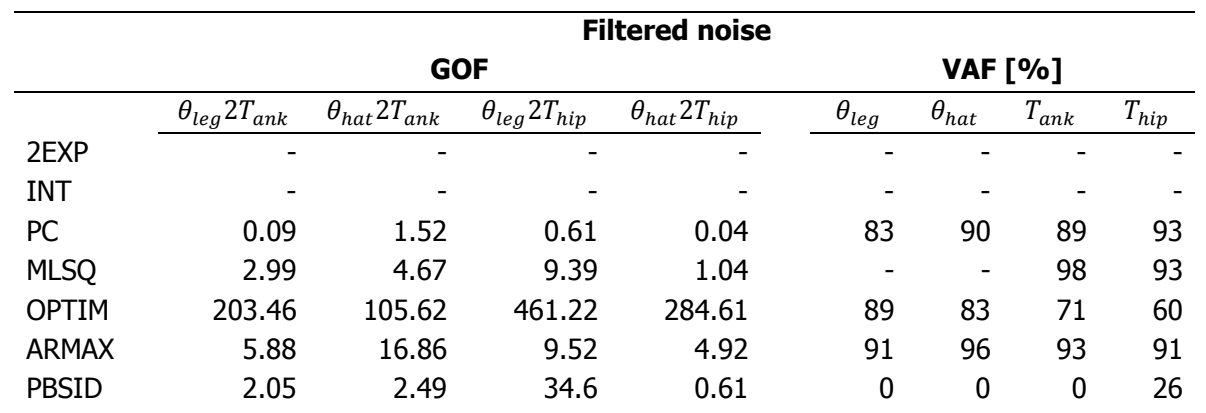




\subsection{Discussion}

Based on model simulations, we evaluated various MIMO closed-loop system identification techniques in combination with external disturbance signals. Results indicated that nonparametric techniques with multisine disturbances gave the most accurate estimation in the time (highest variance accounted for) and frequency (lowest goodness of fit) domain of the neuromuscular controller mechanisms. The use of zippered multisine had the advantage that only one experiment had to be performed, in contrast with the multisine disturbance, which required two experiments. Parametric system identification techniques did not estimate all the underlying neuromuscular controller dynamics. Although the time series were estimated reliably, the dynamics in the frequency domain were not fully captured.

\section{Evaluation of system identification techniques}

We evaluated seven methods to estimate the dynamics of the MIMO neuromuscular controller. The methods were divided in two main approaches: 1) non-parametric system identification techniques, and 2) parametric system identification techniques. We evaluated all methods with simulated data using three quantitative evaluation criteria: variance accounted for (VAF), goodness of fit (GOF) and measurement time.

\section{Non-parametric system identification techniques estimated the dynamics of the neuromuscular controller well for various disturbance signals}

Non-parametric spectral techniques can be applied in combination with various disturbance signals, as long as they contained multiple frequencies in the range of interest and the signals were sufficiently exciting the system. A drawback of the 2EXP method is that two experiments are required to estimate the neuromuscular controller dynamics. This inherently assumes that the system dynamics remain equal over the two experiments, which is not always the case (e.g. humans get fatigued, which changes their behaviour or strategy). A drawback of the INT method is the interpolation of the frequency grid, which possibly induces an error in the FRF, in case the excited frequencies are further apart. The choice of disturbance signal depends on the application, and all signals have advantages and disadvantages. We elaborated on this further in the section about disturbance signal choice.

\section{Parametric system identification techniques were difficult to apply in human balance control, due to the assumed model structure and biological noise in the system}

Parametric system identification techniques estimate a structure through excited frequencies; our results showed that this worked best when all frequencies in the range of interest were excited, i.e. filtered noise signals. Nevertheless, the methods performed well while using multisine signals with many excited frequencies. Four parametric system identification techniques were used to analyse the data, of which two were based on estimation of a feedback gain matrix using multiple least squares and optimization techniques. 


\section{Multiple least squares and optimization techniques}

The MLSQ and OPIM techniques have been applied previously to estimate the general control mechanisms in balance control (Barin, 1989; Winter et al., 2001). These studies showed that the postural feedback gains scaled with the disturbance magnitude and type (Park et al., 2004), and changed with age (Speers et al., 2002). In our study, model simulations showed that the MLSQ and OPTIM methods yielded erroneous results in estimating the dynamics of the neuromuscular controller. This effect was expressed the most in the frequency response functions at the higher frequencies, whereas the time domain fits were quite good. This misfitting in the frequency domain was probably due to the fact that the feedback gain matrix assumed that the underlying mechanisms of the neuromuscular controller consisted of springs and dampers. This is, however a simplification; the model neuromuscular controller also included time delays and muscleactivation dynamics, which were not captured in these estimation methods.

\section{Prediction Error Methods}

Furthermore, we have evaluated a Prediction Error Method (ARMAX) and a Predictor-Based Subspace Identification (PBSID) method. A disadvantage of parametric methods in general is that a model structure was assumed and a specific order was estimated and both the estimation of the order and the model structure can be wrong. For example, an ARMAX model has a dependent structure for the noise and system model (Ljung, 1999). If the estimation of the noise model is incorrect, this affects the estimation of the system model. For example, the biological pink noise in our system does not match the assumed linear noise model. Furthermore, the division of two parametric estimates (which both contain errors) can give inaccurate results, as the errors are present in the closed-loop estimation. Therefore, it is important to check the residuals of the estimation to verify the correctness of the system and noise model.

ARMAX models have been applied in a previous study (Fujisawa et al., 2005), identifying the MIMO neuromuscular postural control mechanisms. However, the excited frequencies only extended until $0.83 \mathrm{~Hz}$. From our model simulations it was seen that the dynamics of balance control extended to approximately $4 \mathrm{~Hz}$ and therefore in this study, we used a broadband disturbance signal.

\section{Predictor Based Subspace Identification}

The advantage of the PBSID method is that the MIMO neuromuscular controller was estimated in one identification step, without dividing two parametric estimates, reducing the errors in the estimation. A disadvantage of subspace methods is that they were designed to estimate the model structure for the case that all noise sources acting on the system are white. However, biological noise (internal disturbances from the sensory and the motor system) is thought to be of pink noise origin (van der Kooij and Peterka, 2011), which has the highest power in the low frequencies. As we are dealing with this biological noise in the feedback mechanism, obtaining a correct estimation can be a challenge. Nevertheless, subspace identification might be a field of further research, as it allows for the studying variation over time (see the section about time invariance). 


\section{Multisine are an optimal choice of disturbance signals}

We used four different disturbance signals (multisine, zippered multisine, filtered noise, and cyclic filtered noise) and evaluated their effectiveness. Below we discuss why multisine are the optimal choice of disturbance signal.

First, compared to filtered noise signals, multisine disturbances have no leakage errors as each harmonic fits exactly an integer number of times in the multisine signal (Pintelon and Schoukens, 2012). In case of leakage, power has leaked out to other frequency bins, which affects the FRF estimate. Secondly, averaging over the repetitive cycles of the disturbance signal reduces noise. Therefore the noise-to-signal ratio (NSR) of the multisine signal is lower than the NSR of the filtered noise disturbances. Finally, exciting fewer frequencies improves NSR further, as there is only (and therefore increased) power at the frequencies of interest. Hence, multisine disturbances have higher NSR compared to filtered noise, and this effect is even stronger when using a zippered multisine.

Furthermore, there is a difference in frequency resolution between the signals. If you are dividing the frequencies over multiple disturbances, thereby creating a zippered multisine, the frequency resolution drops. Therefore, a drawback of multisine disturbances, and even more in zippered multisine disturbances, is that only specific frequencies are excited, making it insensitive to what happens between those frequencies; hence very narrow resonance peaks can be missed.

An advantage of the zippered multisine is that only one experimental trial was needed, which reduced the measurement time by two compared to the multisine method.

In sum, the choice of the "optimal" multisine signal is a trade-off between frequency resolution and measurement time and thus depends on the specific research question or assessment goal.

\section{Application of system identification techniques}

This paragraph describes various challenges, limitations, and future perspectives, to implement system identification techniques in a clinical setting.

\section{Assuming time invariance and linear behaviour}

The system identification techniques assume that human balance control is linear and time invariant (LTI), but in real life, human balance control is highly non-linear and changes over time. With the right experiment and using small disturbance amplitudes, human stance behaviour can be assumed linear around the point of equilibrium (upright stance). However, the parts that compose the neuromuscular controller show time-varying behaviour, as changing muscle activation is required for various tasks (de Vlugt et al., 2002; Ludvig et al., 2011) and postural responses typically adapt or habituate when perturbations have various directions and sizes (Keshner et al., 1987; Bloem et al., 1998; Klomp et al., 2014). For example, subjects effectively adapt their sway response in a changing environment by down-weighting the unreliable sensory information and up-weighting the other information sources (Goodworth and Peterka, 2012). Other studies (van Asseldonk et al., 2006; 
Boonstra et al., 2014a, 2014b) showed that in stroke and PD patients, the contribution in balance control between the two legs can be asymmetrical. Posing more weight on the affected leg can alter the controller properties between the legs to remain in an upright stance (Pasma et al., 2012). Finally, there exist changes over time due to performance limitations, like fatigue.

In model simulations, the LTI behaviour is guaranteed, but interpretation of the outcomes in human experiments must be done carefully. In an experiment, the LTI assumption applies to normal subjects using small perturbations applied in the sagittal plane, which somewhat limits the applicability of the model and methods. When a subject changes its balance control response during the course of the experiment, e.g., from responding stiff to slack, this will lead to an inaccuracy in the estimated FRFs (the estimation will be an average of the stiff and slack dynamic behaviour). It is therefore important to instruct participants in a standardized way and check linearity and time invariance of the responses.

\section{Challenges}

With age and disease (e.g., Parkinson's disease) specific problems present in multisegmental balance control. However, a drawback of analysing a system with two segments is that two perturbations are needed to be able to determine balance control parameters. In this paper we have focused solely on mechanical disturbances, which are often aimed to identify deteriorations in the nervous system part of the control and in the strategies used (ankle or hip strategy). In addition, sensory disturbances can be used to quantify the visual, proprioceptive, and vestibular contributions to maintain standing balance (Peterka, 2002; Jeka et al., 2006; Pasma et al., 2012).

Another aspect that is not captured so far is the influence of deteriorated cognitive control on the balance control behaviour, which is especially an issue in stroke survivors, the elderly, and PD patients (Teasdale and Simoneau, 2001; Doumas et al., 2009; Ambrose et al., 2013; Stijntjes et al., 2015). This effect could be assessed by having participants perform a dual task while maintaining their balance.

\section{Future perspectives to get the methods to the clinic}

In our opinion, system identification techniques could add to the currently used repertoire of balance tests in the clinic by providing a quantitative estimate of the balance control system. However, the methods need to be developed further before they can be used. That is, to bring the system- identification method into clinics, it is first necessary to prove clinical usefulness. Hence, the reliability, sensitivity, and specificity of system identification techniques must be determined and compared to existing clinical balance tests. Subsequently, the ultimate proof is to select a random subject from a healthy young population, a healthy elderly population, and a population with a known deterioration of a specific underlying system and apply system identification techniques to trace from which group this subject was selected. 
Acceptance of these system identification techniques in the clinical field will be challenging as the frequency response functions (FRF) are not directly related to the mechanisms involved in balance control. The FRF shows the behaviour of the system, but does not reveal which physiological mechanisms are underlying the neuromuscular controller. Hence, by fitting a model to the estimated FRF, the outcome measures are now specific parameters with a physiological meaning and the changes in FRF can be related to changes in stiffness or time delays. A prerequisite for reliable physiological parameters is a reliable FRF, which is not self-evident as we have shown in this study.

Finally, it needs to be determined whether the proposed methods are more effective than the current clinical practice: Evaluation of cost-effectiveness and long-term benefits for the patient in terms of quality of life should be an inherent part of the evaluation process.

\subsection{Conclusion}

System identification is the art and science of building mathematical models of dynamical systems (Ljung, 2010). In this paper we showed the "science," e.g. the basic principles of various system identification techniques and the "art," e.g. system identification techniques aimed at application of multi-segmental and closed-loop balance control. Model simulations showed that non-parametric system identification techniques are favourable over parametric estimates in identification of the neuromuscular controller in standing balance. Both multisine signals and filtered noise signals can be used to estimate these dynamics reliably, in which multisine signals have the advantage to excite specific frequencies of interest and therefore have a better NSR. By this overview of the applicability, advantages and disadvantages of the various currently available system identification techniques, a step is made toward applying system identification techniques to detect age and disease-related changes in balance control.

\subsection{Appendix: Standing balance control model}

To validate the MIMO and closed loop system identification techniques, the underlying mechanisms of standing balance control were described in a model structure. Figure 3.1 presents a simplified model of standing balance control, expressed in a plant (i.e. the biomechanics of the human body) and a neuromuscular controller. Each system was described by a mathematical formula (transfer function) with parameters describing the physiology.

\section{Plant: Biomechanics of the human body}

The rigid body dynamics are represented by a double inverted pendulum with the sum of corrective joint torques ( $T_{\text {ank }}$ and $T_{\text {hip }}$ ) and external force disturbances ( Force $_{1}$ and Force $_{2}$ ) as input, and the segment angles $\left(\theta_{\text {leg }}\right.$ and $\left.\theta_{\text {hat }}\right)$ as output. The equations of motion of this double inverted pendulum were derived with the TMT method (Schwab, 1998). The equations of motion were linearized with a Taylor approximation and rewritten in state space (Boonstra et al., 2013). 


\section{Neuromuscular controller: Intrinsic feedback}

The controller is partly based on intrinsic feedback, describing the muscle and tendon dynamics together with the soft tissue properties. These viscous-elastic properties are modelled by a spring (stiffness $\mathrm{K}$ ) in series with a damper (D), acting on the states; i.e. the segment angles and angular velocities. Intrinsic properties are sometimes called passive properties. Although the name "passive" suggests otherwise, this feedback mechanism can be modulated by co-activation of antagonistic muscle groups and therefore the parameters of stiffness and damping can vary. The transfer function is described by:

$$
H_{p}=K_{p}+D_{p} s
$$

In which $s$ is the Laplace operator. The ankles and hips were assumed to have different passive properties $\left(H_{p}^{a n k}, H_{p}^{h i p}\right)$

\section{Neuromuscular controller: Reflexive feedback}

Intrinsic feedback alone is not sufficient to maintain balance. Therefore, the central nervous system (CNS) continuously generates motor commands to compensate for the unstable body dynamics, which is called reflexive feedback control and results in phasic muscle activation. Information to the CNS originates from noisy data (modelled by pink biological noise) from the proprioceptive, visual and vestibular system.

Reflexive feedback $\left(H_{r}\right)$ was represented by a matrix with stiffness and damping terms, relating the joint torques to the segment angles and angular velocities. This resulted in four transfer functions.

$$
\begin{aligned}
& H_{r}^{\theta_{\text {leg } 2 T_{\text {ank }}}}=K_{\theta_{\text {leg } 2 T_{\text {ank }}}}+D_{\theta_{\text {leg } 2 T_{\text {ank }}} \mathrm{S}} \\
& H_{r}^{\theta_{\text {hat }} 2 T_{\text {ank }}}=K_{\theta_{\text {hat }} 2 T_{\text {ank }}}+D_{\theta_{\text {hat }} 2 T_{\text {ank }}} \mathrm{S} \\
& H_{r}^{\theta_{l e g} 2 T_{h i p}}=K_{\theta_{l e g} 2 T_{\text {hip }}}+D_{\theta_{\text {leg }} 2 T_{\text {hip }}} s \\
& H_{r}^{\theta_{\text {hat }} 2 T_{\text {hip }}}=K_{\theta_{\text {hat }} 2 T_{\text {hip }}}+D_{\theta_{\text {hat }} 2 T_{\text {hip }}} S
\end{aligned}
$$

Reflexive control is delayed due to sensory transduction, transmission, and processing, resulting in a lumped time delay (sum of neural conduction time (transport delay), an electromechanical delay (to activate the muscles), and the processing time of sensory information). Furthermore, the conversion from motor control signals to muscle force is represented by the muscle-activation dynamics. The time delay $\left(\tau_{d}\right)$ and activation dynamics $\left(H_{a c t}\right)$ can be represented by the following transfer functions:

$$
\begin{aligned}
& H_{T D}=e^{-\tau_{d} s} \\
& H_{a c t}=\frac{\omega^{2}}{s^{2}+2 \beta \omega s+\omega^{2}}
\end{aligned}
$$

In which $\omega$ and $\beta$ the natural frequency and relative damping of the muscle activation dynamics. For both the ankle and hip joint, different values were chosen $\left(H_{T D}^{a n k}, H_{T D}^{h i p}, H_{a c t}^{a n k} H_{a c t}^{h i p}\right)$. 
The entire neuromuscular controller $\left(H_{c}\right)$ can be expressed in four transfer functions:

$$
\begin{gathered}
H_{c, \theta_{\text {leg }} 2 T_{\text {ank }}}=\frac{T_{\text {ank }}}{\theta_{\text {leg }}}=H_{p}^{\text {ank }}+H_{r}^{\theta_{\text {leg } 2 T} T_{\text {ank }}} H_{T D}^{\text {ank }} H_{a c t}^{\text {ank }} \\
H_{c, \theta_{\text {hat }} 2 T_{\text {ank }}}=\frac{T_{\text {ank }}}{\theta_{\text {hat }}}=H_{r}^{\theta_{\text {hat }} 2 T_{\text {ank }}} H_{T D}^{a n k} H_{a c t}^{\text {ank }} \\
H_{c, \theta_{\text {leg }} 2 T_{\text {hip }}}=\frac{T_{\text {hip }}}{\theta_{\text {leg }}}=H_{r}^{\theta_{\text {leg } 22 T_{\text {hip }}} H_{T D}^{\text {hip }} H_{a c t}^{\text {hip }}} \\
H_{c, \theta_{\text {hat }} 2 T_{\text {hip }}}=\frac{T_{\text {hip }}}{\theta_{\text {hat }}}=H_{p}^{\text {hip }}+H_{r}^{\theta_{\text {hat }} 2 T_{\text {hip }}} H_{T D}^{\text {hip }} H_{\text {act }}^{\text {hip }}
\end{gathered}
$$

\section{Parameter settings}

Parameters for the plant and controller need to be set in order for the model to work (Table 3.3). Parameters for body segments are mass, length, height of the CoM above the lower end of the segments and the moment of inertia about the lower end of the segment. When simulating at a multi-segmented model, the equations of motion in the plant are separated for a lower segment (legs) with a torque around the ankle and an upper segment (hat) with a torque around the hip. The two joints have different controller properties; intrinsic properties and also the time delays and activation dynamics were different between the

\begin{tabular}{|c|c|c|}
\hline Body parameter value & $\begin{array}{l}\text { Lower } \\
\text { (legs) }\end{array}$ & $\begin{array}{l}\text { Upper segment } \\
\text { (HAT) }\end{array}$ \\
\hline Mass $[\mathrm{kg}]$ & 20.70 & 48.00 \\
\hline Length $[\mathrm{m}]$ & 0.83 & - \\
\hline Height of the CoM above lower end [m] & 0.52 & 0.30 \\
\hline Moment of inertia $\left[\mathrm{kg} \mathrm{m}^{2}\right]$ & 6.57 & 7.07 \\
\hline Disturbance height relative to CoM [m] & 0.40 & 0.44 \\
\hline \multicolumn{3}{|l|}{ Intrinsic feedback } \\
\hline Intrinsic stiffness [Nm/rad] & 286.00 & 149.00 \\
\hline Intrinsic damping [Nms/rad] & 65.60 & 24.80 \\
\hline \multicolumn{3}{|l|}{ Reflexive feedback } \\
\hline Transport delay [s] & 0.06 & 0.04 \\
\hline Angular eigen frequency [rad/s] & 9.90 & 12.10 \\
\hline Damping fraction $[-]$ & 1.50 & 1.65 \\
\hline Neural controller & $K[\mathrm{Nm} / \mathrm{rad}]$ & $\mathrm{D}[\mathrm{Nms} / \mathrm{rad}]$ \\
\hline$\theta_{\text {leg }} 2 T_{\text {ank }}$ & 567.16 & 236.80 \\
\hline$\theta_{\text {hat }} 2 T_{\text {ank }}$ & 291.82 & 108.91 \\
\hline$\theta_{\text {leg }} 2 T_{\text {hip }}$ & 153.38 & 107.08 \\
\hline$\theta_{\text {hat }} 2 T_{\text {hip }}$ & 159.94 & 105.88 \\
\hline
\end{tabular}
segments. These parameters are described by Kiemel et al (Kiemel 2011). In all simulations, the data were collected with a sample frequency of $1 \mathrm{kHz}$.

Table 3.3: Parameter settings for the model simulations 


\title{
Chapter 4
}

\section{Assessment of multi-joint coordination and adaptation in standing balance: A novel device and system identification technique}

Denise Engelhart, Alfred C. Schouten, Ronald G.K.M. Aarts, and Herman van der Kooij. IEEE TNSRE, 2014 Nov 20, epub ahead of print

\begin{abstract}
The ankles and hips play an important role in maintaining standing balance and the coordination between joints adapts with task and conditions, like the disturbance magnitude and type, and changes with age. Assessment of multi- joint coordination requires the application of multiple continuous and independent disturbances and closed loop system identification techniques (CLSIT). This paper presents a novel device, the double inverted pendulum perturbator (DIPP), which can apply disturbing forces at the hip level and between the shoulder blades. In addition to the disturbances, the device can provide force fields to study adaptation of multi-joint coordination. The performance of the DIPP and a novel CLSIT was assessed by identifying a system with known mechanical properties and model simulations. A double inverted pendulum was successfully identified, while force fields were able to keep the pendulum upright. The estimated dynamics were similar as the theoretical derived dynamics. The DIPP has a sufficient bandwidth of $7 \mathrm{~Hz}$ to identify multijoint coordination dynamics. An experiment with human subjects where a stabilizing force field was rendered at the hip $(1500 \mathrm{~N} / \mathrm{m})$, showed that subjects adapt by lowering their control actions around the ankles. The stiffness from upper and lower segment motion to ankle torque dropped with $30 \%$ and $48 \%$ respectively. Our methods allow to study (pathological) changes in multi-joint coordination as well as adaptive capacity to maintain standing balance.
\end{abstract}




\subsection{Introduction}

Balance in daily life is constantly challenged by both external forces, such as gravity and pushes, and by internal disturbances such as noisy sensory signals and motor commands, and movements of limbs involved in other motor tasks. To prevent falling, the neuromuscular controller generates corrective joint torques to keep the body upright, based on proprioceptive, vestibular and visual feedback (Peterka, 2003). During stance, balance is mainly regulated by the ankle and hip joints, which involves multi-joint coordination. Multijoint coordination in stance is usually expressed in terms of the ankle strategy and hip strategy, which are defined as movements in these joints (Horak and Nashner, 1986; Creath et al., 2005). However, the observed movements are caused by both corrective joint torques and by the internal and external disturbances. To identify the neuromuscular controller, closed loop system identification techniques (CLSIT) (van der Kooij et al., 2005; Engelhart et al., 2014) can be applied to reveal the causal relation from movements of the lumped legs and head-arms-trunk (hat), to corrective joint torques in the ankle and hip.

When becoming older, multi-joint coordination changes (Accornero et al., 1997; Gariépy et al., 2008). To maintain standing balance in various situations, postural responses must adapt with task, magnitude and type of external disturbance (Alexandrov et al., 2001b; Park et al., 2004; Kim et al., 2012). Studies in the upper and lower extremities showed that the neuromuscular controller also adapted when external force fields were applied (Shadmehr and Mussa-Ivaldi, 1994; Burdet et al., 2001; Noël et al., 2008; van Asseldonk et al., 2009). How multi-joint coordination in stance depends on such force fields, may provide insight in the adaptive capacity to maintain standing balance. To our best knowledge the use of external force fields has not been applied in balance control during stance.

The application of CLSIT to study both multi-joint coordination and adaptation during stance, requires an independent disturbance for each input of the neuromuscular controller (Pintelon and Schoukens, 2012). In this specific case, the inputs are the segment angles of the leg and hat, and therefore two independent disturbances must be applied that continuously challenge the balance control system (de Vlugt et al., 2003; Pintelon and Schoukens, 2012; Boonstra et al., 2013). Several studies do not apply disturbances at all (Kuo et al., 1998; Alexandrov et al., 2001b; Speers et al., 2002), or only one disturbance (Park et al., 2004; Alexandrov et al., 2005; Kim et al., 2009, 2012; Goodworth and Peterka, 2012), which could lead to incorrect identification of the neuromuscular controller (Boonstra et al., 2013). Only a few studies investigated the multivariate nature of balance control by applying two disturbances (Fujisawa et al., 2005; Kiemel et al., 2011; Boonstra et al., 2013).

Researchers used custom built apparatuses to apply these two disturbances at various places on the human body. The study of Fujisawa et al (Fujisawa et al., 2005) described an apparatus in which disturbance signals were applied to the lumbar level (L1) and to the legs about $3 \mathrm{~cm}$ below the hip joint. The body segments were linked to a stepping motor through an elastic string, which pulled the trunk forward and the legs backward. Disturbing the human body in a specific direction is predictable and possibly does not capture full 
neuromuscular control dynamics. Kiemel et al (Kiemel et al., 2011) applied two mechanical disturbances to the subject from behind, at waist and shoulder level. Both disturbances were applied by a spring with one end attached to a waist/shoulder strap worn by the subject and the other end attached to a linear motor placed directly behind the subject. However, a spring can only pull the body, thereby only provoking corrective joint torques in one direction. Boonstra et al (Boonstra et al., 2013) applied disturbances by support surface translation with a movable platform and an actuated backboard at the level of the hips. The actuated backboard was able to apply perturbing torques in both the anterior and posterior direction at the sacrum. A drawback of the experimental set-up lies with the platform disturbance. Accelerations of the movable platform disturb the human body, but the directly following accelerations in the opposite direction can have a stabilizing effect (van Asseldonk et al., 2007).

Most multi-joint coordination studies also have some limitations of the CLSIT approach, mainly due to their choice of disturbance signal. In the study of Fujisawa (Fujisawa et al., 2005) the bandwidth of the disturbances was only $0.83 \mathrm{~Hz}$, whereas to the dynamics of the human body extents until approximately $4 \mathrm{~Hz}$ (van der Kooij and de Vlugt, 2007). Human experiments are limited in measurement time as subjects become fatigued. It is advantageous to use a periodic disturbance signal and to apply this signal multiple times. Averaging over the periods leads to a reduction of noise. In the case of Kiemel (Kiemel et al., 2011), white noise disturbances were applied that are not periodic. This resulted in long measurement times, as averaging over experiments was performed. The method of Boonstra (Boonstra et al., 2013) applied periodic multisine signals. However, the two disturbances have equal frequency content and to make the disturbances independent, two experiments were needed with different sets of disturbances.

This paper describes the Double Inverted Pendulum Perturbator (DIPP), a novel forcecontrolled manipulator to assess multi-joint coordination during standing balance. Two independent manipulators can push and pull the subject at hip and shoulder level with a bandwidth of $7 \mathrm{~Hz}$, which is sufficient to identify the neuromuscular controller. In addition to the disturbances, the device can provide force fields which possibly evokes adaptation. To study both multi-joint coordination and adaptation we present a CLSIT approach, that offers the advantages of the periodic multisine signals and needs only a single experiment. 


\subsection{Methods}

This section first describes the design and control of the DIPP. Secondly, the CLSIT algorithm is presented. To technically validate the apparatus, a double inverted pendulum (2IP) was attached to the DIPP and external force fields were applied to keep the 2IP upright. Validity of the CLSIT is proven by model simulations and by comparing the identified 2IP dynamics to theoretical 2IP dynamics. Finally, the application of the DIPP and the CLSIT is demonstrated by identifying multi-joint coordination in humans and adaptation of multi-joint coordination to external force fields.

\section{Apparatus}

\section{Design}

The DIPP is a custom made (Forcelink B.V., The Netherlands) device to apply forces at the pelvis and between the shoulder blades (Figure 4.1). Each manipulator was actuated by a rotating electromotor (ABI; type LSH050-4-60-320) with an amplifier (LTI type CDE300). The motor drives a metal rod through a thin steel wire connected to both ends of the rod.
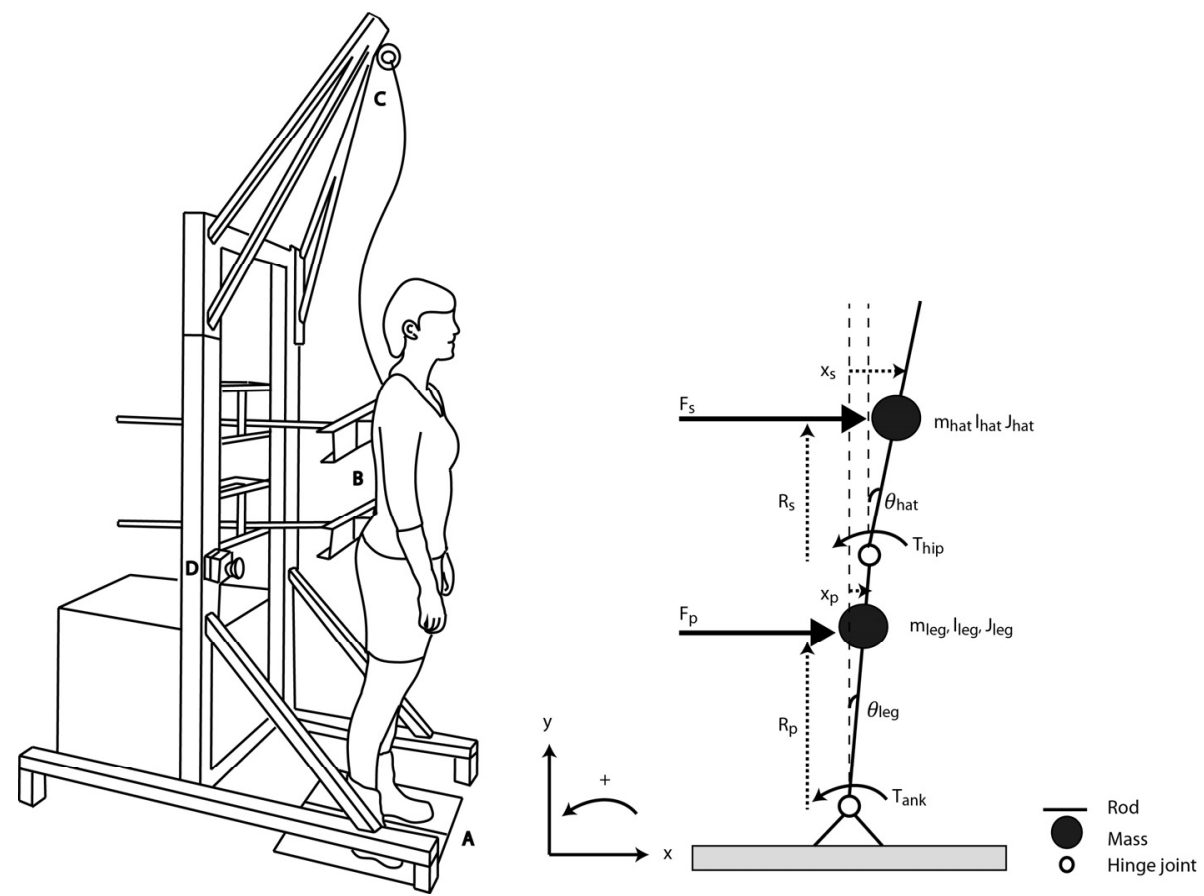

Figure 4.1: Left: Double Inverted Pendulum Perturbator (DIPP) with a subject standing on a force plate (A) and attached to the manipulators at shoulder and hip level (B). For safety, a harness is attached to the pyramidal construction (C) and an emergency button is mounted on the frame (D). Right: an ideal physical model of a double inverted pendulum (2IP) connected to the DIPP. Masses are attached to two beams, creating a leg and a hat segment, pivoting around hinge joints (ankle and hip). The 2IP is kept upright by external force fields. The 2IP model also applies to standing balance in humans, where internal torques $\left(T_{\text {ank }}, T_{\text {hip }}\right)$ are generated to keep the human body upright. 
The wire winds around a drum that was connected to the motor axis and thereby transformed motor rotation to rod translation ( 1 full rotation equals $0.1 \mathrm{~m}$ translation). The subject was strapped to the rods via a board with memory foam, which molded quickly to the shape of the body and allowed pushing and pulling the subject. Both manipulators (including the motors) were adjustable in height such that the rod was aligned at the subject's hip and shoulder level. For testing purposes, the setup can also be used to stabilize and identify a mechanical double inverted pendulum (2IP). The motors were controlled using a PC with a PCI-card (National Instruments; NI PCI- 6229) running xPC-target (The Mathworks, MA) at $10 \mathrm{kHz}$.

Load cells (Zemic Europe; type BG3-C3-100kg-6B) were placed between the rod and the board to measure the force exerted on the subject. Two draw-wire potentiometers (Sentech; type Celesto SP2-25) were attached to the end of the rod and measured the displacement. The pretension of $0.02 \mathrm{~N}$ in the draw-wire was negligible and did not influence rod or body movement. The subject stood on a dual force plate (AMTI; Watertown USA), measuring the ground reaction forces and torques in six degrees of freedom. A safety harness prevented the subject from falling.

\section{Control}

The manipulators were force controlled such that force disturbances and force fields could be implemented. Force disturbances are pushes and pulls on the human body, required for system identification purposes. Force fields refer to altered dynamics of the DIPP, and possibly evoke adaptation of multi-joint coordination. Subjects experience force fields as being attached to e.g. a spring, altering the dynamical interaction with the environment. Figure 4.2 shows the general control scheme of the DIPP.

In the motor controller, the velocities of the manipulators were controlled by velocity servos. The velocity servos were tuned in the amplifiers of the motors and the proportional and integral (PI) values were set such that sufficient bandwidth of around $40 \mathrm{~Hz}$ was obtained. The motors were tuned without a human connected, but including the metal rod and load cell.

To apply force disturbances, the pelvis and shoulder manipulators were controlled independently. In Figure 4.2 the settings for the shoulder are indicated with subscript $s$ and the settings of the pelvis are indicated with subscript $p$. In order to drive the plant (human or 2IP) with forces, the motor controller input is the difference between the reference forces $\left(F_{r s}, F_{r p}\right)$ and the measured forces $\left(F_{s}, F_{p}\right)$ from the load cells. A proportional controller $\left(P_{s}, P_{p}\right)$ converts this force difference into the velocity input $\left(v_{r s}, v_{r p}\right)$ of the velocity servo. The proportional gain was determined from the Ziegler-Nichols rules in closed loop. A human subject was attached to the device and the gain was increased until the measured force signal started to oscillate. Half of this gain value resulted in a proportional gain of $0.005 \mathrm{~m} / \mathrm{Ns}$, for both the shoulder and pelvis manipulator and remained fixed for all subjects and measurements. The velocity servo, rotates with a certain speed $\left(v_{s}, v_{p}\right)$ and results in translation of the rod $\left(x_{s}, x_{p}\right)$. 
A) General Control Scheme

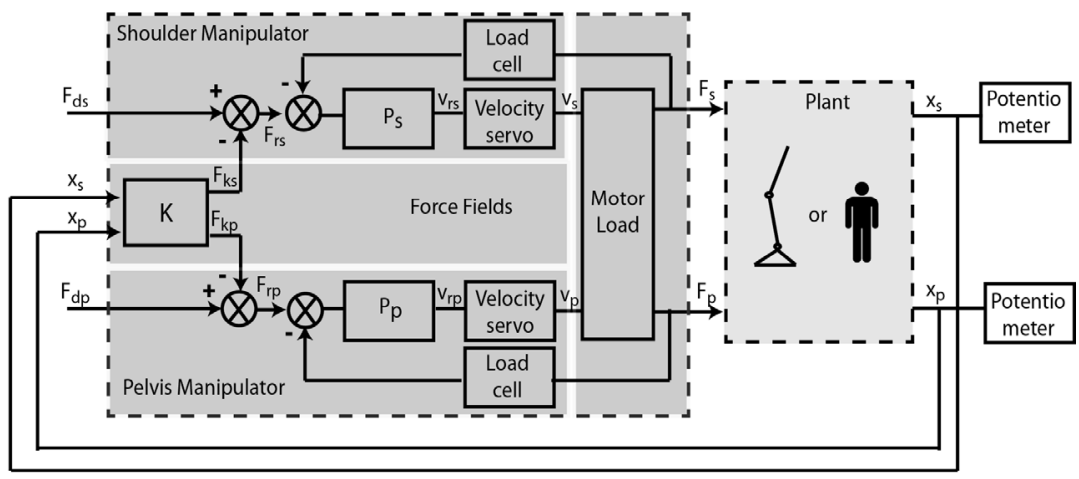

B) Human

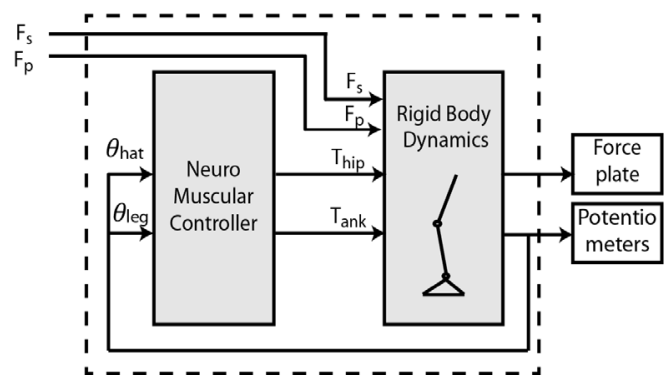

Figure 4.2: A) General control scheme of the DIPP. Force reference signals $\left(F_{r s}, F_{r p}\right)$, result from external disturbances $\left(F_{d s}, F_{d p}\right)$ and added stiffness $\left(F_{k s}, F_{k p}\right)$. The forces are controlled by means of a $\mathrm{P}$ controller. The velocity servo interacts with a motor load, resulting in a forces $\left(F_{s}, F_{p}\right)$ applied to the plant. The plant can either be a double inverted pendulum (2IP) or the human body. B) In case of a human plant, an internal neuromuscular controller regulates the upright position of the rigid body dynamics (comparable to a 2IP). Ground reaction forces are measured with a force plate from which joint torques $\left(T_{\text {ank }}, T_{\text {hip }}\right)$ are calculated with inverse dynamics. In addition, segment angles $\left(\theta_{\text {leg }}, \theta_{\text {hat }}\right)$ are calculated from the displacement measured with the potentiometers.

Force fields were implemented in the controller as virtual springs, written with a stiffness matrix K:

$$
F_{k}(t)=\left[\begin{array}{l}
F_{k p}(t) \\
F_{k s}(t)
\end{array}\right]=-\left[\begin{array}{cc}
k_{p} & k_{s 2 p} \\
k_{p 2 s} & k_{s}
\end{array}\right]\left[\begin{array}{l}
x_{p}(t) \\
x_{s}(t)
\end{array}\right]=-K x(t)
$$

The rod translations were converted to a (virtual) spring force $\left(F_{k p}, F_{k s}\right)$ using the $\mathrm{K}$ matrix. The stiffness can be either set directly (diagonal terms) from the shoulder position to the shoulder force $\left(k_{s}\right)$ or from the pelvis position to the pelvis force $\left(k_{p}\right)$. Furthermore, interaction was possible by indirect stiffness (off-diagonal terms) from position of the pelvis to the shoulder force $\left(k_{p 2 s}\right)$ and vice versa $\left(k_{s 2 p}\right)$. In this paper, only direct stiffness was applied. 


\section{Safety}

Safety was implemented at three levels to assure safety of the subjects; a software, electronic and hardware level. For each level, the safety bounds become more restrictive. Software limits were set in XPC target, which restricts the magnitude of the position and the force signals to $\pm 0.2 \mathrm{~m}$ and $\pm 55 \mathrm{~N}$, respectively. Furthermore, the velocity was limited to $\pm 0.45 \mathrm{~m} / \mathrm{s}$. Finally power was limited to $15 \mathrm{~J} / \mathrm{s}$, as force applied at large velocity can also be harmful. Within the electronic circuit of the velocity servo, the maximum speed was limited to $300 \mathrm{rpm}$ (equivalent to $\pm 0.5 \mathrm{~m} / \mathrm{s}$ ). The maximum torque was limited to $0.95 \mathrm{Nm}$ ( $\approx$ $60 N)$. As ultimate safety, some hardware restrictions exist. The maximum movement of the rod was limited by its length of $0.7 \mathrm{~m}$. An emergency button - in reach of both the subject and the experimenter - interrupts the power supply when pressed, and the subject wore a safety harness to prevent falling.

\section{Data recording and processing}

During all measurements, motor reference velocities, forces from the load cells, and movement of the rods were recorded at $1 \mathrm{kHz}$ and stored on a PC for further processing. The leg and hat segment angles of the subject or 2IP with respect to the upright position were calculated using the rod positions and goniometric rules. In case of the human subject, the forces and torques from the dual force plate were recorded and the ankle and hip torque were calculated from the force plate data, with inverse dynamics (Schwab, 1998).

\section{Closed Loop System Identification Technique}

A closed-loop system identification technique (CLSIT) was used to separately estimate the dynamics of any subsystem in the motor controller and the plant (van der Kooij et al., 2005; Pintelon and Schoukens, 2012). Here, a CLSIT is presented, using the advantages of periodic multisine. So called 'zippered' multisine are applied, in which the disturbances have separate frequency content. Only one measurement trial is needed as the disturbance signals are naturally independent.

\section{Disturbance signals}

For controller tuning, multisine disturbances of $20 \mathrm{~s}$ were applied with in a range from 0.05 $10 \mathrm{~Hz}$. Only the odd harmonics were excited, and the frequencies were linearly spaced. The disturbances were repeated 18 times giving 6 minutes of data. In the identification experiments of the 2IP and the human, multisine disturbances of $20 \mathrm{~s}$, in which the odd harmonics are logarithmically distributed, were applied in a range from $0.05-5 \mathrm{~Hz}$. From each set of 3 odd harmonics, one was randomly assigned to the pelvis disturbance, one was randomly assigned to the shoulder disturbance and the third one was not used. Consequently, the shoulder and pelvis disturbances were independent. The disturbances were repeated 9 times. In all measurements, the first repetition cycle was discarded to remove possible transient effects. 


\section{Algorithm}

The estimated dynamics in a closed loop were expressed by means of a frequency response function (FRF) (de Vlugt et al., 2003). The FRF displays the differences between the responses and the disturbances in means of amplitude and time, given in a magnitude and a phase curve, respectively. In general form the FRF is given by:

$$
H(f)=S_{d y}(f) S_{d u}(f)^{-1}
$$

In which $f$ are the frequencies in the disturbance signal and $S_{d y}(f)$ and $S_{d u}(f)$ are the cross spectral density matrices between the external disturbances (d) and the input (u) and the output $(\mathrm{y})$ signals of $\mathrm{H}$ respectively.

$$
\begin{aligned}
& S_{d y}(f)=\left[\begin{array}{ll}
S_{d 1 y 1}(f) & S_{d 1 y_{2}}(f) \\
S_{d 2 y 1}(f) & S_{d 2 y 2}(f)
\end{array}\right] \\
& S_{d u}(f)=\left[\begin{array}{ll}
S_{d 1 u 1}(f) & S_{d 1 u^{2}}(f) \\
S_{d 2 u 1}(f) & S_{d 2 u 2}(f)
\end{array}\right]
\end{aligned}
$$

To obtain these matrices, the recordings were segmented in the repetitions of the disturbance signals and consecutively transformed to the frequency domain. The required cross spectral densities were calculated and averaged to reduce noise. Figure 4.3 shows that inverting the $S_{d u}(f)$ matrix and multiplying it with the $S_{d y}(f)$ matrix requires that the matrix components are known at all excited frequencies, which is untrue for the zippered multisine, as both input signals contain different frequencies.

Therefore, the complex numbers of the cross spectral densities were interpolated in terms of magnitude and phase, to obtain all matrix components for the full range of excited frequencies in the zippered multisine. The FRFs were only evaluated at the frequencies where the disturbance signals contained power.

To validate the performance and validity of the DIPP and CLSIT, the FRF of the force loop and the plant (2IP) were estimated by:

$$
\begin{gathered}
H_{\text {force }}(f)=S_{d F}(f) S_{d F r}(f)^{-1} \\
H_{\text {plant }}(f)=S_{d x}(f) S_{d F}(f)^{-1}
\end{gathered}
$$

To estimate the dynamic behaviour of the human neuromuscular controller, the FRF from segment angles $(\theta)$ to joint torques $(\mathrm{T})$ was obtained.

$$
H_{n m c}(f)=-S_{d T}(f) S_{d \theta}(f)^{-1}
$$




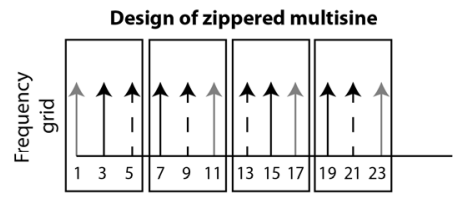

Frequency Response Function

Interpolation of spectral densities

$$
\begin{aligned}
& \mathrm{H}=\mathrm{S}_{\mathrm{dy}} \mathrm{S}_{\mathrm{du}}{ }^{-1} \\
& S_{d y}=\left[\begin{array}{ll}
S_{d_{1 y 1}} & S_{d_{1 y 2}} \\
S_{d_{2 y 1}} & S_{d_{2} y_{2}}
\end{array}\right] \\
& S_{d_{u}}=\left[\begin{array}{ll}
S_{d_{1} u_{1}} & S_{d_{1} u_{2}} \\
S_{d_{2} u_{1}} & S_{d_{2} u_{2}}
\end{array}\right]
\end{aligned}
$$
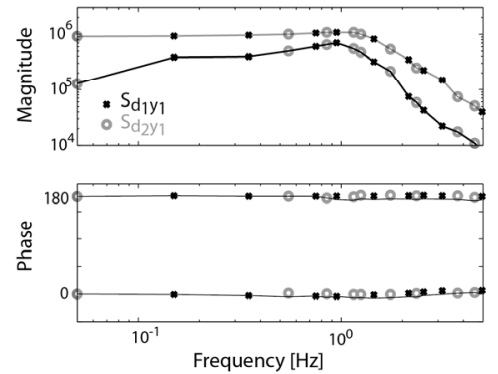

Figure 4.3: Left: Design of a zippered multisine in which the odd harmonics (multiples of the base frequency $0.05 \mathrm{~Hz}$ ) are distributed over the pelvis (grey) and shoulder (black) grid. Middle: The spectral densities are only known on either the frequencies of the pelvis or shoulder disturbance. Right: Obtaining all matrix components requires interpolation of the magnitude and phase. An example is shown here for the 2IP.

In addition to the FRF, the coherence was estimated, which indicates the amount of random variation in each Fourier component of the signal. The squared coherence was calculated from the disturbances to the ankle and hip angles and torques.

$$
\begin{aligned}
& \gamma_{d \theta}(f)^{2}=\left|\mathrm{S}_{d \theta}(f)\right|^{2}\left[\mathrm{~S}_{d d}(f) \mathrm{S}_{\theta \theta}(f)\right]^{-1} \\
& \gamma_{d T}(f)^{2}=\left|\mathrm{S}_{d T}(f)\right|^{2}\left[\mathrm{~S}_{d d}(f) \mathrm{S}_{T T}(f)\right]^{-1}
\end{aligned}
$$

The squared coherence has a value between 0 and 1 . The closer the value reaches 1 , the lower the noise level and the more the system behaviour can be considered time invariant.

\section{Performance of the device}

To test the performance of the DIPP, a double inverted pendulum (2IP) with known dynamics was build. The 2IP (Figure 4.1) consisted of two beams (both $3 \mathrm{~kg}$ ), with two attached masses (both $5 \mathrm{~kg}$ ), connected by hinge joints. Additional parameters for the 2IP are given in Table 4.1. The pelvis manipulator was attached to the lower segment and the shoulder manipulator was attached to the upper segment at the level of the weights. To counter the effects of gravity and keep the 2IP upright, force fields were applied as stiffness of $1500 \mathrm{~N} / \mathrm{m}$.

The performance of the DIPP is described by the force loop. $H_{\text {force }}$ was determined in an experimental setting where the 2IP or a human subject were attached to the DIPP. Ideally, the magnitude of the force loop equals 1 ; then the disturbance forces are sufficiently applied to the plant. The bandwidth of the force loop was defined as the lowest frequency where the magnitude decreased by $3 \mathrm{~dB}$, corresponding to a magnitude of 0.7 . 
Table 4.1: Parameter Values for the Double Inverted Pendulum

\begin{tabular}{clc}
\hline Parameter & \multicolumn{1}{c}{ Description } & Value \\
\hline$l_{\text {leg }}$ & Length of the leg segment & $1.06 \mathrm{~m}$ \\
$l_{\text {hat }}$ & Length of the hat segment & $0.80 \mathrm{~m}$ \\
$R_{p}$ & Height of the pelvis manipulator with respect to the ankle hinge joint & $0.75 \mathrm{~m}$ \\
$R_{s}$ & Height of the shoulder manipulator with respect to the hip hinge joint & $0.33 \mathrm{~m}$ \\
$m_{\text {leg }}$ & Mass of the leg segment & $8.00 \mathrm{~kg}$ \\
$m_{\text {hat }}$ & Mass of the hat segment & $8.00 \mathrm{~kg}$ \\
$d_{\text {leg }}$ & Distance of the center of mass with respect to the ankle hinge joint & $0.67 \mathrm{~m}$ \\
$d_{\text {hat }}$ & Distance of the center of mass with respect to the hip hinge joint & $0.36 \mathrm{~m}$ \\
$J_{\text {leg }}$ & Inertia of the leg segment & $0.37 \mathrm{kgm}^{2}$ \\
$J_{\text {hat }}$ & Inertia of the hat segment & $0.17 \mathrm{kgm}^{2}$
\end{tabular}

\section{Validity of the CLSIT}

The validity of the CLSIT was verified in two cases. First, the dynamics of the 2IP $\left(H_{\text {plant }}\right)$ were estimated and compared to the theoretical FRF. The linearized equations of motion of the 2IP were derived (Schwab, 1998) and implemented in Matlab symbolic toolbox. The inputs were the applied force disturbances and the outputs were the segment displacements.

Secondly, in case of a human subject, the plant not only consists of rigid body dynamics similar as the 2IP, but also has an internal neuromuscular controller (Figure 4.2b) (van der Kooij et al., 2005). The properties of this neuromuscular controller are unknown and can adapt to various tasks and conditions. To demonstrate that the CLSIT is valid to identify the dynamics of the neuromuscular controller, a computational model of the human rigid body dynamics and neuromuscular controller was used. Time series of angles and joint torques were generated by computer simulations. Based on these time series CLSIT was applied to estimate the FRF of the neuromuscular controller $\left(H_{n m c}\right)$ and compared to the theoretical FRF of the model. The model is described in detail in the Appendix.

\section{Adaptation of balance control}

5 healthy young volunteers ( 3 male / 2 female, $24 \pm 2$ years old, $83 \pm 19 \mathrm{~kg}$ ) participated in an experiment and their neuromuscular controller $\left(H_{n m c}\right)$ was identified with and without a force field. In the experiments with a force field, a stiffness of $k_{p}=1500 \mathrm{~N} / \mathrm{m}$ was rendered at the pelvis. The order of the experiments with and without force field were randomized per subject. We hypothesized that application of a stabilizing force field would lead to downscaling of postural responses. We expect that subjects adapt their postural response mainly for low frequencies $(<1 \mathrm{~Hz})$, where the stiffness manifests. The average magnitude of the neuromuscular controller for this frequency range was calculated and termed effective stiffness. A paired t-test was performed on the effective stiffness to test the effect of a stabilizing force field. 


\subsection{Results}

\section{Performance of the device}

As both manipulators are similar, only the results of the shoulder manipulator are presented. The magnitude of the force loop for both the 2IP and the human is close to unity up to the bandwidth of approximately $7 \mathrm{~Hz}$ (Figure 4.4). This is sufficient to estimate the behaviour of the human neuromuscular controller, as we only excite the system (and thereby identify the dynamics) with frequencies below $5 \mathrm{~Hz}$.

\section{Validity of the CLSIT}

First, the validity of the CLSIT was verified by identification of a double inverted pendulum (2IP). Using a virtual stiffness to keep the 2IP upright and by application of two disturbances, the dynamic behaviour of the 2IP was derived. Figure 4.5 shows that the identification closely resembles the theoretical FRF of the 2IP, which shows the validity of the CLSIT, also when external force fields were simultaneously applied.

Secondly, the validity of the CLSIT to identify the human neuromuscular controller was verified using numerical model simulations, in which the plant not only consisted of rigid body dynamics, but also of a neuromuscular controller. Similar DIPP motor settings and disturbances were used as in the 2IP experiments. Figure 4.6 shows that the estimated and theoretical FRF of the neuromuscular control are identical, which shows the validity of the CLSIT to identify the neuromuscular controller.

\section{Adaptation of balance control}

Experimental data is shown in Figure 4.7. Humans responded similar over the 9 periods in the disturbances and time series were averaged over periods. The coherences were all above 0.8 in each subject, for all responses. This indicates that the noise level is reasonable low and subjects behave relatively time invariant.

Figure 4.8 shows the estimated FRF of the neuromuscular controller. The magnitude of the neuromuscular controller of the ankle was highest for low frequencies and decreased above $2 \mathrm{~Hz}$. The magnitude of the neuromuscular controller of the hip torque was roughly constant over frequencies and increased above $2 \mathrm{~Hz}$. The phases of all neuromuscular controller FRFs decreased with frequency, indicating the presence of a neural time delay. Effective stiffness around the ankle torque was found significantly lower in the force field condition; $\theta_{\text {leg }} 2 \mathrm{~T}_{\text {ank }}$ ( $30 \%$ decrease, $\mathrm{p}=0.001)$ and $\theta_{\text {hat }} 2 \mathrm{~T}_{\text {ank }}$ ( $48 \%$ decrease, $\left.\mathrm{p}=0.008\right)$. Rendered stiffness at the hip did not change the effective stiffness around the hip torque $\left(\theta_{\operatorname{leg}} 2 \mathrm{~T}_{\text {hip }}, \mathrm{p}=0.55\right.$; $\left.\theta_{\text {hat }} 2 \mathrm{~T}_{\text {hip }}, \mathrm{p}=0.22\right)$. 

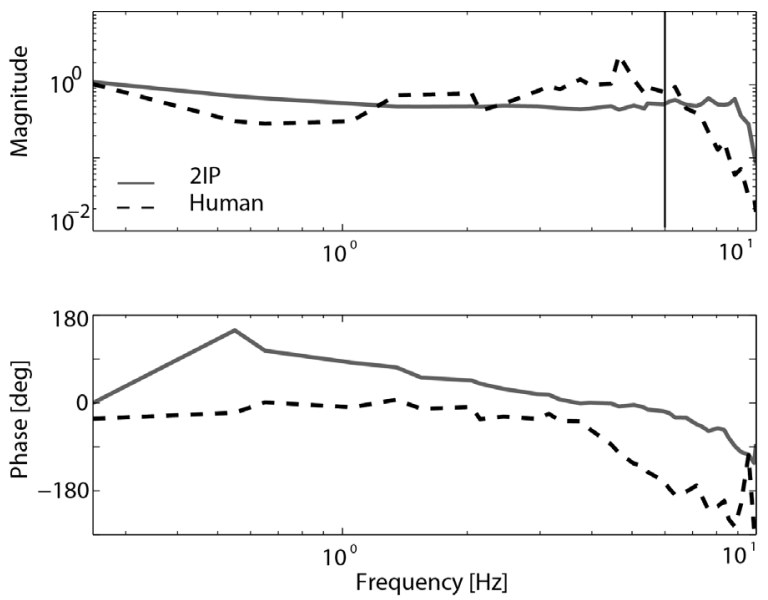

Figure 4.4: Frequency response function of the force loop when a 2IP (solid gray line) or a typical subject is attached (dashed black line). Data was averaged over 17 repetitive cycles of the disturbance signal. The bandwidth of $7 \mathrm{~Hz}$ is indicated with the vertical line.
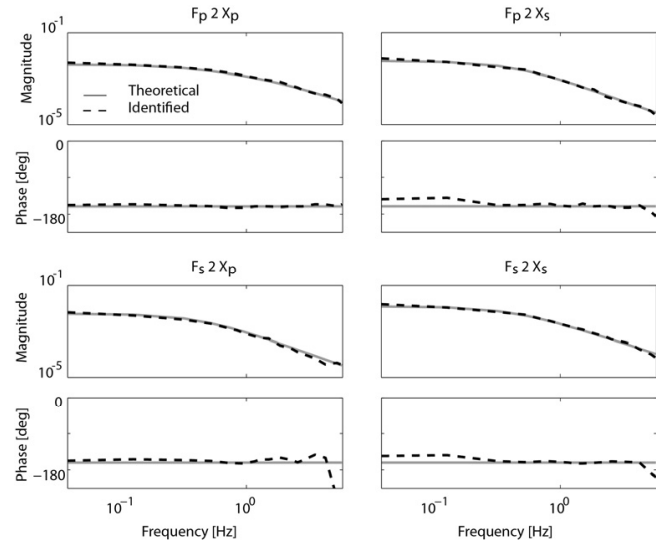

$\mathrm{F}_{\mathrm{s}} 2 \mathrm{X}_{\mathrm{s}}$
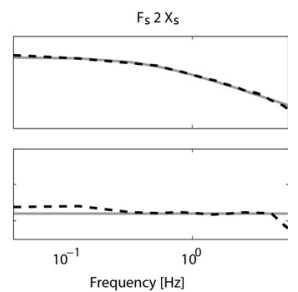

Figure 4.5: Identification of the dynamics of the 2IP. The FRF consists of four terms, two direct terms from pelvis force to pelvis position $\left(F_{p} 2 X_{p}\right)$ and from shoulder force to shoulder position $\left(F_{s} 2 X_{s}\right)$, and two indirect terms from pelvis force to shoulder position $\left(F_{p} 2 X_{s}\right)$ and from shoulder force to pelvis position $\left(F_{s} 2 X_{p}\right)$. The theoretical FRF is given by the solid gray line. The identified FRF is given by the dashed black line.
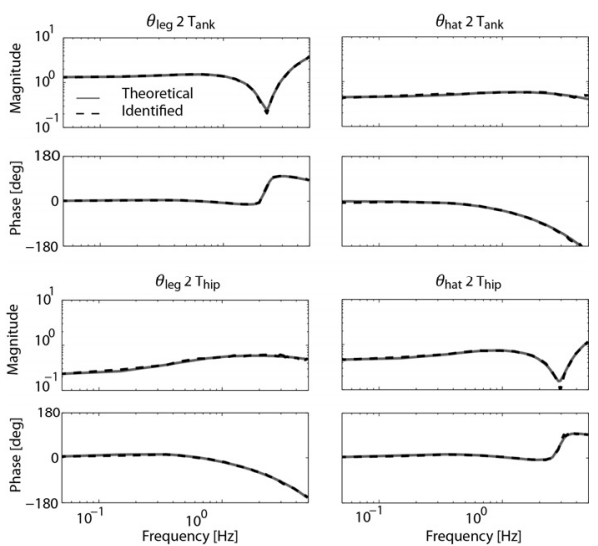

Figure 4.6: Identification of the dynamics of the human neuromuscular controller in model simulations. The FRF consists of four terms, two direct terms from leg angle to ankle torque $\left(\theta_{\text {leg }} 2 T_{\text {ank }}\right)$ and from hat angle to hip torque $\left(\theta_{\text {hat }} 2 T_{\text {hip }}\right)$, and two indirect terms from hat angle to ankle torque $\left(\theta_{\text {hat }} 2 T_{\text {ank }}\right)$, and from leg angle to hip torque $\left(\theta_{\text {leg }} 2 T_{\text {hip }}\right)$. The theoretical FRF is given by the solid gray line. The identified FRF is given by the dashed black line. 

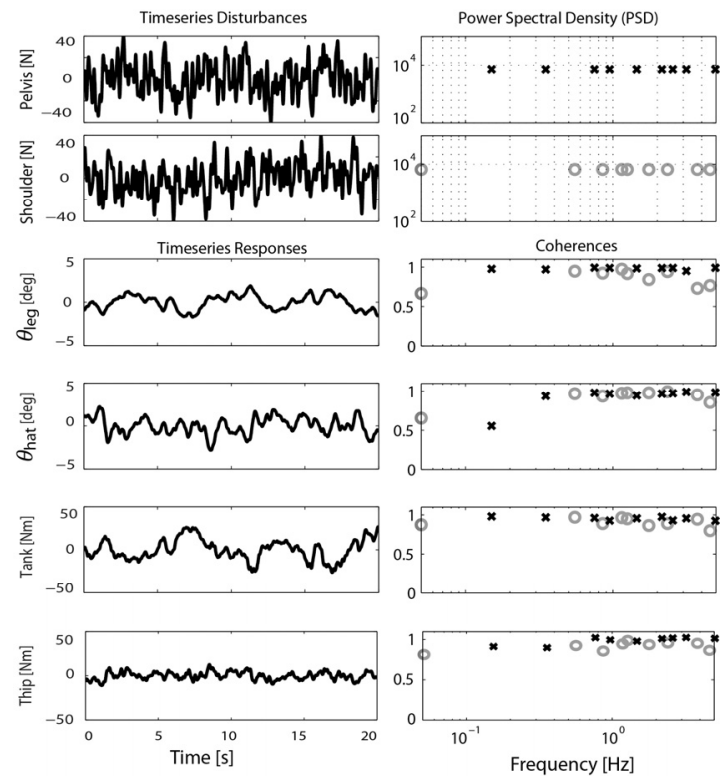

Figure 4.7: Experimental data from a typical subject, in which two disturbances were applied without additional force field. Upper part: the time series of the manipulator forces $\left(F_{s}, F_{p}\right)$ with the corresponding power spectral density. Lower part: the human leg and hat angles $\left(\theta_{\text {leg }}, \theta_{\text {hat }}\right)$ and ankle and hip torque $\left(T_{\text {ank }}, T_{\text {hip }}\right)$, with corresponding coherences. Data was averaged over 8 repetitive cycles of the disturbance signal.
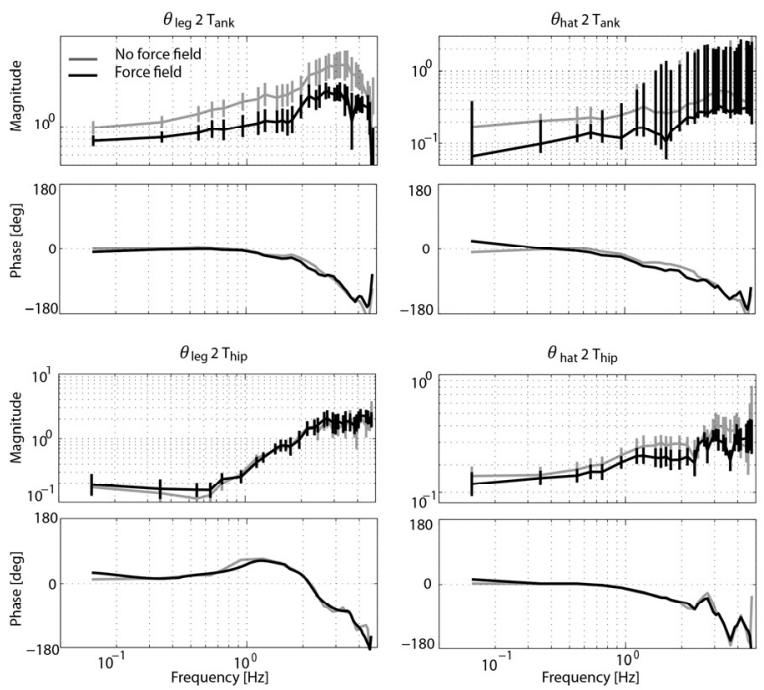

Figure 4.8: Average identified human neuromuscular controller of the participants, without (solid gray line) or with (dashed black line) a stabilizing force field (stiffness of $1500 \mathrm{~N} / \mathrm{m}$ ) rendered at the pelvis. Standard errors are indicated with error bars. 
Although few subjects were included in this study, application of external force fields at the hip showed that subjects adapted by lowering their control action at the ankle. With the force fields subjects were externally stabilized, reducing the required balance maintenance.

\subsection{Discussion}

The neuromuscular control properties in multi-joint balance control are described by the dynamic relations between stabilizing ankle and hip torques as a response to movement of the legs and hat. As there is interaction between the segments, such a relation can only be established by applying two independent disturbances in combination with closed loop system identification techniques (CLSIT). Our aim was to develop a device which allows to investigate multi-joint coordination in standing balance and adaptation of the neuromuscular control mechanisms. We also presented a CLSIT based on zippered multisine, to study both multi-joint coordination and adaptation, with shorter measurement time compared to previously used techniques.

\section{Apparatus}

In standing balance control, force disturbances are common in daily life situations. Subjects can actively control their upright position to maintain stance, in the presence of the pull of gravity and internal and external disturbances.

To identify multi-joint coordination, for each degree of freedom, an additional disturbance is required, which is not acknowledged in all studies. The DIPP can apply two independent push and pull disturbances with frequencies up to $7 \mathrm{~Hz}$, which is sufficient to capture the neuromuscular controller dynamics of the ankle and the hips in the sagittal plane, which are present until approximately $4 \mathrm{~Hz}$.

A unique feature of the DIPP is the ability to apply force fields, simultaneous with the force disturbances. Virtual springs can be applied with varying stiffness to induce adaptation of multi-joint coordination.

\section{CLSIT}

Force disturbances involve interaction between the subject and the DIPP, creating a closed loop system. Therefore, system identification techniques which incorporates this closed loop are required. The use of standard open-loop algorithms in closed loop, introduce bias in the estimates (van der Kooij et al., 2005). There are multiple available methods, which properly use two disturbances and closed loop system identification techniques (CLSIT) (Fujisawa et al., 2005; Kiemel et al., 2011; Boonstra et al., 2013), but they differ from the presented method in this paper. The study of Fujisawa applies a parametric CSLIT. Parametric estimates can separate noise from the system, but requires knowledge about the underlying system structure. The other methods are non-parametric, which do not require prior knowledge about the system. The study of Kiemel applies white noise signals, which requires more data to reduce noise and for each experimental condition, 12 trials of 250 seconds each are recorded. To reduce the measurement time, period multisine signals can 
be applied. Having power at specific frequencies increases the participant's responses and thereby decreases measurement time. In the method of Boonstra and colleagues, the same frequency grid is applied in each disturbance signal. To make the signals independent, two experiments are performed. In the first experiment, both inputs are excited with the same excitation, while in the second experiment the sign of the second input is changed. Our method only requires one experiment, as both disturbances have a different frequency grid. A disadvantage of this method is reduced frequency resolution in the disturbances. Interpolation to the full frequency grid makes it possible to apply CLSIT.

In general, the obtained FRFs are in the same range as other studies. In the study of Fujisawa, comparison is only possible for low frequencies as very low bandwidth disturbances $(0.83 \mathrm{~Hz})$ were applied. However, applying disturbances with a different setup, at different application locations or by using different signals could have elicited different responses (Kim et al., 2012).

\section{Application}

The DIPP allows to study multi-joint coordination in standing balance, as well as adaptation to external force fields.

Ageing is associated with impaired balance and falls (Pasma et al., 2014b). Previous studies show that elderly have altered strategies to maintain standing balance compared to young adults. Previous studies show that elderly exhibit higher cross-correlation between the upper and lower body movements in quiet stance, indicating decreased variability between the two body segments (Accornero et al., 1997; Gariépy et al., 2008), which possibly indicates that elderly are stiffer than young adults (Gariépy et al., 2008). Identifying the underlying cause of impaired balance is difficult, as the neuromuscular controller is known to have substantial redundancy at the joint, muscle and neural levels (Hsu et al., 2013). Understanding the quality and contribution of the underlying mechanisms in standing balance would aid to detect individuals with a high risk of falling and would help to set up targeted interventions. By applying two independent force disturbances at the hip and shoulder using the DIPP and applying CLSIT, multi-joint coordination in standing balance can be quantified. Differences between elderly and young subjects in neuromuscular control properties of the ankle and the hips can possibly explain altered balance control mechanisms.

Poor adaptation to changing environments also increases fall risk. To study adaptation in multi-joint coordination, the DIPP allows to apply external force fields, manipulating the movements around the ankles and hips. How multi-joint coordination in standing depends on such force fields, possibly shows adaptive capacity to maintain standing balance. In this study, spring stiffness (i.e. force fields) acts as a stabilizing force and experimental pilot data suggests that subjects downscale their postural responses. The magnitude of the neuromuscular controller, expressed by the effective stiffness, decreased around the ankle joint for added force fields. 


\subsection{Conclusion}

In this paper a double inverted pendulum perturbator (DIPP) and a closed loop system identification technique (CLSIT) were presented to study multi-joint coordination during stance. A human experiment was performed showing adaptation of multi-joint neuromuscular control properties to force fields. Our methods allow to study pathological changes in multi-joint coordination as well as adaptive capacity to maintain standing balance. In different populations e.g. elderly with impaired balance, Parkinson's disease patients or stroke subjects, changes can be detected in the sensory systems, nervous system deteriorations, motor system deteriorations or a combination of these factors.

\subsection{Appendix}

This section describes the human balance control model (Figure 4.9), as it was used in the computer simulations. The model consist of rigid body dynamics and a neuromuscular controller.

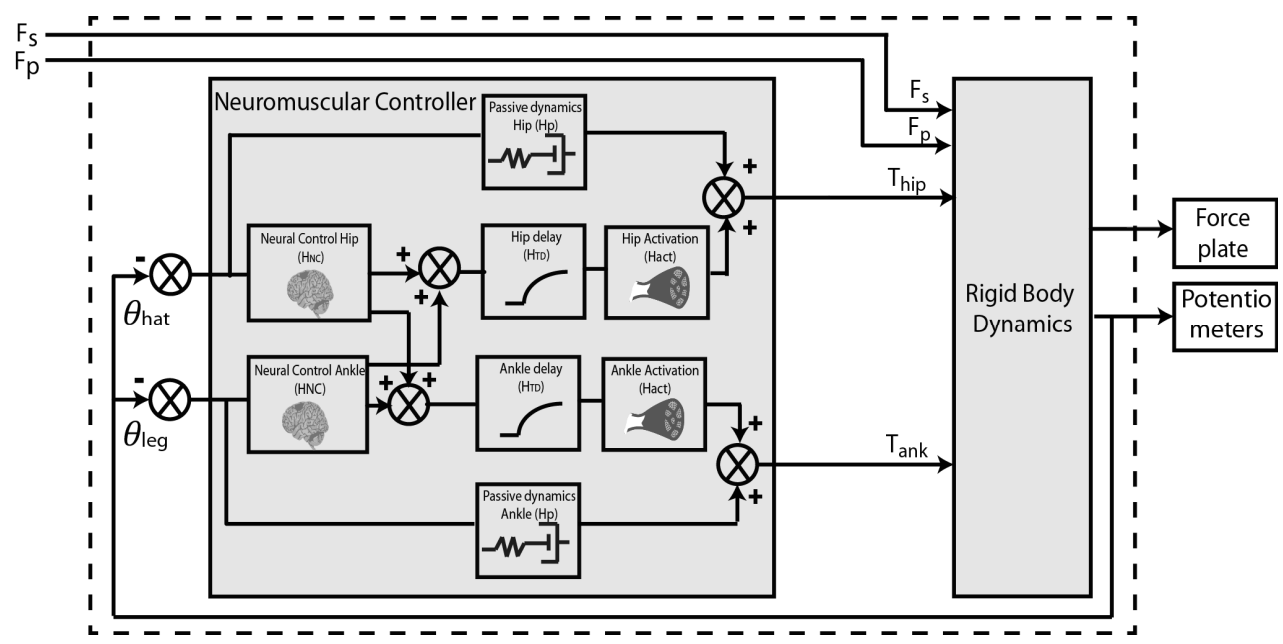

Figure 4.9: Simplified representation of standing balance control

\section{Rigid Body Dynamics}

The rigid body dynamics are described by a double inverted pendulum (2IP). The inputs are the corrective joint torques resulting from the neuromuscular controller and the external force disturbances. The output are the angles of the leg and hat. The equations of motion of this 2IP can be described with the TMT method (Schwab, 1998). The equations of motion are linearized with a Taylor approximation and rewritten in state space.

$$
\begin{aligned}
& \dot{x}=A x+B u \\
& y=C x+D u
\end{aligned}
$$


With $\mathrm{x}=\theta_{\text {leg }}, \theta_{\text {hat }}, \dot{\theta}_{\text {leg }}, \dot{\theta}_{\text {hat }}$, the systems states (segment angles and angular velocities). The inputs $u$ of the system are the applied force disturbances. The outputs $y$ are the segment displacements.

\section{Neuromuscular controller: Passive feedback}

The neuromuscular controller is partly based on intrinsic feedback, describing the muscle and tendon dynamics together with the soft tissue properties. These viscous-elastic properties were modelled by a spring (stiffness $\mathrm{K}$ ) in series with a damper (D), acting on the states; the joint angles and angular velocities. Although the name 'passive' suggest otherwise, these passive feedback mechanisms can be modulated by co-activation of antagonistic muscle groups and therefore the parameters of stiffness and damping can vary. The transfer function is described by:

$$
H_{p}=K+D s
$$

\section{Neuromuscular controller: Active feedback}

Passive feedback alone is not sufficient to maintain the body in the upright position. Therefore, the Central Nervous System (CNS) continuously generates motor commands to compensate for the unstable body dynamics, which is called active feedback control and results in phasic muscle activation. Information to the CNS originates from noisy sensory data, which makes control challenging. For simulations, the neural controller was represented as an LQR controller (Kuo, 2005), where the motor command is dependent of the positions and velocities of the body-segments (states). To deal with the noise information, this controller uses an internal, forward model of body and sensory dynamics to predict sensory output. Feedback gains $\left(K_{B S}\right)$ for stabilizing the body are determined by minimizing a parameterized objective function which is subject to the systems dynamics.

$$
\hat{x}_{B S}=A x_{B S}+B u_{\text {control }}
$$

In case of a discrete-time state-space model with states $x_{B S}$, the feedback control signal $\left(u_{\text {control }}\right)$ becomes:

$$
u_{\text {control }}=-K_{B S} x_{B S}
$$

In which $K_{B S}$ is a $2 \times 4$ matrix, with gains from the 4 states $\left(x_{B S}\right)$ to the 2 motor commands $\left(u_{\text {control }}\right)$ as outputs. Therefore, $H_{N C}$ influences both ankle and hip joints.

$$
\left[\begin{array}{c}
T_{\text {ank }} \\
T_{\text {hip }}
\end{array}\right]=-\left[\begin{array}{llll}
k_{11} & k_{12} & k_{13} & k_{14} \\
k_{21} & k_{22} & k_{23} & k_{24}
\end{array}\right]\left[\begin{array}{c}
\theta_{\text {leg }} \\
\theta_{\text {hat }} \\
\omega_{\text {leg }} \\
\omega_{\text {hat }}
\end{array}\right]
$$

Neural control is delayed due to sensory transduction, transmission and processing, resulting in a time delay. Furthermore, the conversion from motor control signals to muscle force is represented by the muscle activation dynamics. 
The time delay $\left(H_{T D}\right)$ and activation dynamics $\left(H_{\text {act }}\right)$ can be represented by the following transfer functions:

$$
\begin{gathered}
H_{T D}=e^{-\tau_{d} s} \\
H_{a c t}=\frac{\omega^{2}}{s^{2}+2 \beta \omega s+\omega^{2}}
\end{gathered}
$$

In which $\tau_{d}$ is the transport delay, $\omega$ and $\beta$ the eigen-frequency and relative damping of the muscle activation dynamics.

The entire neuromuscular controller $\left(H_{n m c}\right)$ can be expressed in four transfer functions:

$$
\begin{aligned}
& H_{n m c, \theta_{\text {leg }} 2 T_{\text {ank }}}=\frac{T_{\text {ank }}}{\theta_{\text {leg }}}=H_{p}^{\text {ank }}+H_{N C}^{\theta_{\text {leg }} 2 T_{\text {ank }}} H_{T D}^{\text {ank }} H_{\text {act }}^{\text {ank }} \\
& H_{n m c, \theta_{\text {hat }} 2 T_{\text {ank }}}=\frac{T_{\text {ank }}}{\theta_{\text {hat }}}=H_{N C}^{\theta_{\text {hat }} 2 T_{\text {ank }}} H_{T D}^{\text {ank }} H_{\text {act }}^{\text {ank }} \\
& H_{n m c, \theta_{\text {leg }} 2 T_{\text {hip }}}=\frac{T_{\text {hip }}}{\theta_{\text {leg }}}=H_{N C}^{\theta_{\text {leg }} 2 T_{\text {hip }}} H_{T D}^{\text {hip }} H_{\text {act }}^{\text {hip }} \\
& H_{n m c, \theta_{\text {hat }} 2 T_{\text {hip }}}=\frac{T_{\text {hip }}}{\theta_{\text {hat }}}=H_{p}^{\text {hip }}+H_{N C}^{\theta_{\text {hat }} 2 T_{\text {hip }}} H_{T D}^{\text {hip }} H_{\text {act }}^{\text {hip }}
\end{aligned}
$$

\section{Parameter settings}

Parameters for the plant and neuromuscular controller need to be set in the model. Parameters for body segments are mass, length, height of the body Centre of Mass (CoM) above the lower end of the segments and the moment of inertia around the lower end of the segment. When simulating a multi-segmented model, the equations of motion in the plant were separated for a lower segment (legs) with a torque around the ankle and an upper segment (hat) with a torque around the hip. The two joints have different controller properties; intrinsic properties and also the time delays and activation dynamics were altered between the segments. These parameters are described by two prior studies(Kiemel et al., 2011; Boonstra et al., 2013). Time delays are based on literature describing longlatency responses of the upper and lower body (Nashner, 1976; Matthews, 1991; Henry et al., 1998; Safavynia and Ting, 2013). All model simulations were performed in Matlab Simulink, with a sample frequency of $100 \mathrm{~Hz}$. 
Table 4.2: Parameter Values for the Double Inverted Pendulum

\begin{tabular}{lcc}
\hline & leg segment & hat segment \\
\hline Body parameter value & & \\
Mass [kg] & 20.70 & 48.00 \\
Length [m] & 0.83 & - \\
Height of the CoM above lower end [m] & 0.52 & 0.30 \\
Moment of inertia [kg m²] & 6.57 & 7.07 \\
Disturbance height relative to CoM [m] & 0.40 & 0.44 \\
Passive feedback & & \\
Intrinsic stiffness [Nm/rad] & 286.00 & 149.00 \\
Intrinsic damping [Nms/rad] & 65.60 & 24.80 \\
Active feedback & & \\
Transport delay [s] & 0.10 & 0.08 \\
Angular eigen frequency [rad/s] & 9.90 & 12.10 \\
Damping fraction [-] & 1.50 & 1.65 \\
Neural controller & Angle \\
$\theta_{\text {leg }} 2 \mathrm{~T}_{\text {ank }}$ & [Nm/rad] & Angular velocity \\
$\theta_{\text {hat }} 2 \mathrm{~T}_{\text {ank }}$ & 608.33 & [Nms/rad] \\
$\theta_{\text {leg }} 2 \mathrm{~T}_{\text {hip }}$ & 305.96 & 249.89 \\
$\theta_{\text {hat }} 2 \mathrm{~T}_{\text {hip }}$ & 154.43 & 114.55 \\
& 163.16 & 108.05 \\
& & 106.71
\end{tabular}




\title{
Chapter 5
}

\section{Adaptation of multi-joint coordination during standing balance in healthy young and healthy old individuals}

\author{
D. Engelhart, J.H. Pasma, A.C. Schouten, R.G.K.M. Aarts, C.G.M. Meskers, A.B. Maier, H. \\ van der Kooij. Submitted
}

\begin{abstract}
Standing balance requires multi-joint coordination between the ankles and hips. We investigated how humans adapt their multi-joint coordination to adjust to various conditions and prevent from falling and whether this differed with age. Fifteen healthy young participants between 20-30 years of age and 14 healthy elderly above 70 years of age participated in the study. Balance was disturbed by push/pull rods, applying two continuous and independent force disturbances at the level of the hip and between the shoulder blades. In addition, external force fields were applied, represented by an external stiffness at the hip, either stabilizing or destabilizing the participants' balance. Multivariate closed-loop system identification techniques were used to obtain a description of the neuromuscular control mechanisms by quantifying the corrective joint torques as a response to body sway, represented by Frequency Response Functions (FRFs). Model fits on the FRFs resulted in an estimation of time delays, intrinsic stiffness, reflexive stiffness, and reflexive damping, both of the ankle and hip joint. The elderly generated similar corrective joint torques but had reduced body sway compared to the younger participants, which corresponded to the increased FRF magnitude with age. When a stabilizing or destabilizing external force field was applied at the hip, both young and elderly participants adapted their multi-joint coordination by lowering or respectively increasing their neuromuscular control actions around the ankles, expressed in a change of FRF magnitude. However, the elderly adapted less compared to the young participants. Model fits on the FRFs showed that although the elderly and the young participants had comparable reflexive stiffness and reflexive damping, the elderly adapted their reflexive stiffness around the ankle joint less. Furthermore, the elderly showed higher intrinsic stiffness of the ankle, together with higher time delays of the hip. These results imply that elderly were stiffer and were less able to adapt to external force fields. These age-related differences in adaptation of multi-joint coordination may underlie increased risk of falling with ageing.
\end{abstract}




\subsection{Introduction}

Ageing is associated with impaired balance and falls (Rubenstein, 2006; Muir et al., 2010; Pasma et al., 2014b). To maintain balance, several underlying systems work together. One of the mechanisms to maintain balance is to alter the coordination of postural responses, i.e. multi-joint coordination, which is often explored by analyzing ankle strategies and hip strategies (Horak and Nashner, 1986), describing the movement in these joints. Coordination of movements around the ankle and hip joints depends on the amount of external disturbances - such as gravity and pushes having impact on the body - and on the support surface conditions (Horak and Nashner, 1986; Creath et al., 2005; Fujisawa et al., 2005). Adaptability of multi-joint coordination is an essential feature of standing balance control in order to adjust to various conditions.

Previous studies showed that the elderly tend to have altered multi-joint coordination to maintain standing balance compared to young individuals. The elderly exhibited higher cross-correlation between the upper and lower body in quiet stance, indicating that the displacements of the two body segments were less independent (Accornero et al., 1997; Gariépy et al., 2008). In addition, the elderly had a less-flexible joint coordination pattern to compensate for externally applied balance disturbances (Hsu et al., 2013). This possibly indicates that the elderly used less hip strategy and behaved more rigidly.

Various underlying mechanisms have been put forth as contributing factors of altered multijoint coordination in the elderly. Some studies indicated that the elderly increase their reflexive stiffness around the ankle joint when they are exposed to altered sensory information (Amiridis et al., 2003; Benjuya et al., 2004), while other studies found altered intrinsic properties of muscles and tendons having impact on stiffness in the elderly (Kearney et al., 1997; Ishida et al., 2008; Cenciarini, 2010).

Detecting the underlying mechanisms of altered multi-joint coordination in standing balance is complex, as there exists substantial redundancy at the joint, muscle, and neural levels (Hsu et al., 2013). Multiple sensory systems contribute to balance control, i.e. the proprioceptive, visual and vestibular system. The sensory signals are integrated and processed by the central nervous system and are then used to generate corrective joint torques by precise muscle-activation patterns. Due to this redundancy, cause and effect remain unclear; increased stiffness can be due to altered intrinsic muscle properties or increased reflex activity, or it might be a compensation strategy and a result of the fact that the elderly change their dynamic behaviour to maintain stability. Multivariate closed-loop system identification techniques (CLSIT) (Boonstra et al., 2013; Engelhart et al., 2014) are required to unravel cause and effects. Applying multiple and specifically designed external disturbances on the human body allows the study of multi-joint coordination in standing balance. Multi-joint coordination is expressed in the generation of corrective joint torques as a response to body movement; i.e. the dynamic behaviour of the neuromuscular controller.

With system identification techniques, we investigated the underlying mechanisms of multijoint coordination in standing balance in healthy young and healthy elderly. Furthermore, we 
investigated the adaptation to externally applied force fields. Studies in the upper and lower extremities showed that postural responses adapted when external force fields were applied (Shadmehr and Mussa-Ivaldi, 1994; Burdet et al., 2001; Franklin et al., 2003; van Asseldonk et al., 2009). In standing balance studies, it was shown that the elderly possesses the ability to adapt to external disturbances, although to a lesser degree than the young (Pavol et al., 2002; Ooteghem et al., 2009). Therefore, we hypothesize that a stabilizing force field will downscale the postural responses and that this effect is more pronounced in the young than the elderly, indicating the elderly are less adaptive. In addition to existing studies, identification of the underlying mechanisms that change multi-joint coordination with age, could provide insight into factors influencing the risk of falling in the elderly (Engelhart et al., 2014). Adequate treatment of balance disorders requires unraveling the underlying primary causes and applied (adaptive) strategies.

\subsection{Methods}

\section{Participants}

Fifteen healthy young (age range 20-30 years) and 14 healthy elderly individuals (age range 70-79 years) participated in the study (Table 5.1). Participants were excluded when they: 1) were in a dependent living situation; 2) were unable to walk a distance of $250 \mathrm{~m}$; 3) presented co-morbidity (dementia, neurologic disorders, metabolic diseases, rheumatic diseases; heart failure; severe chronic obstructive pulmonary disease; 4) used medication with an influence on balance control (immunosuppressive drugs, insulin, anticoagulation); 5) were immobilized for one week during the last three months or 6 ) had orthopedic surgery during the last two years with unresolved pain or functional limitation.

To illustrate the state of health of the study population, several outcome parameters were measured. Cognition was assessed using the Mini-Mental State Examination (MMSE) (Folstein et al., 1975) and participants with a score lower than 26 points were not included. Physical functioning was assessed by handgrip strength and the Short Physical Performance Battery (SPPB) (Guralnik et al., 1994). Walking speed was obtained from the four meter walking test of the SPPB. The total amount of medication was obtained by questioning the participants.

The study was performed according to the principles of the Declaration of Helsinki and was approved by the Medical Ethics Committee of Medisch Spectrum Twente, Enschede, the Netherlands. All participants gave written informed consent before participating in the study. 
Table 5.1: Participant characteristics. All parameters are presented as mean with standard deviation unless indicated otherwise. Abbreviations: IQR, interquartile range; MMSE, Mini-Mental State Examination; SPPB, Short Physical Performance Battery.

\begin{tabular}{lrr}
\hline & Young $(\mathbf{n = 1 5})$ & Elderly $(\mathbf{n = 1 4})$ \\
\hline General characteristics & & \\
Age, years & $25.9(2.8)$ & $74.4(3.5)$ \\
Women ( $\mathrm{n}, \%)$ & $8(53)$ & $6(43)$ \\
Weight, kg & $71.7(10.4)$ & $78.7(10.6)$ \\
Height, m & $1.80(0.09)$ & $1.72(0.08)$ \\
Health characteristics & & \\
Number of medication, median (IQR) & $0(0)$ & $2(0-5)$ \\
MMSE, points; median (IQR) & $30(29-30)$ & $29(26-30)$ \\
Physical functioning & & \\
Handgrip strength, kg & $50.8(16.75)$ & $36.7(7.8)$ \\
Gait speed, m/s & $1.07(0.16)$ & $1.07(0.15)$ \\
SPPB score, points; median (IQR) & $12(11-12)$ & $12(10-12)$
\end{tabular}

\section{Apparatus}

Multi-joint coordination was investigated using a custom made device (Motekforce Link, Culemborg, The Netherlands), the Double Inverted Pendulum Perturbator (DIPP) (Engelhart et al., in press). The DIPP consists of two manipulators and applies forces using push/pull rods at hip and shoulder level (Figure 5.1). Both manipulators were adjustable in height to align the rods to the participant's hip and shoulder level. The manipulators were force controlled such that force disturbances and force fields could be simultaneously applied. Force disturbances are pushes and pulls on the human body, required for identification of the neuromuscular controller. Force fields were applied at hip level to evoke adaptation of multi-joint coordination. Participants experienced the force fields as if they were attached to a spring with varying stiffness, pushing or pulling them back to an equilibrium position. During the experiments, participants wore a safety harness to prevent falling. The harness did not constrain movements or provide support in any way.

\section{Disturbance signals}

The force disturbances were unpredictable and continuous multisine signals in a range from $0.05-5 \mathrm{~Hz}$ (Figure 5.2). The shoulder and pelvis disturbances were zippered multisine (Pintelon and Schoukens, 2012), such that each disturbance contained nine excited frequencies at an interleaved frequency grid. Both multisine signals had a period of 20 seconds, and were repeated nine times over a time course of three minutes. Disturbances had a peak-to-peak amplitude of $80 \mathrm{~N}$. 


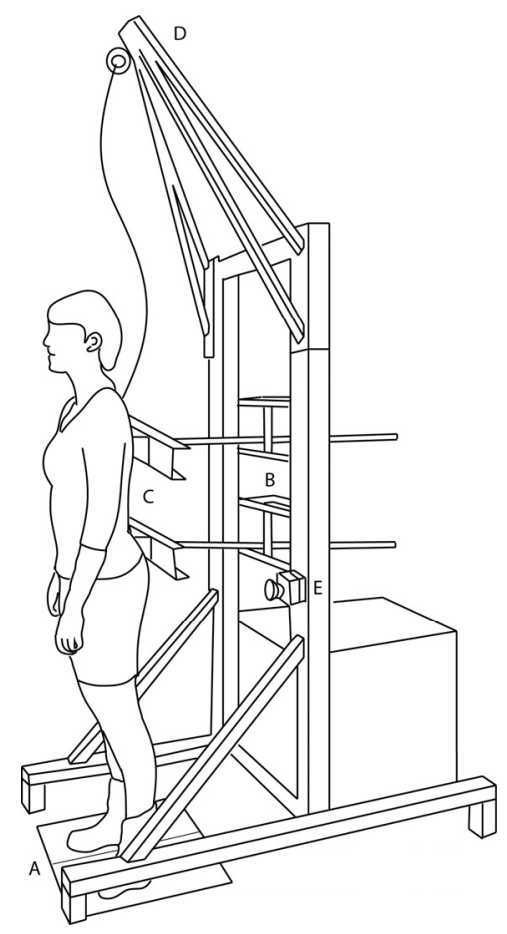

Figure 5.1: Double Inverted Pendulum Perturbator (DIPP) with a subject standing on a force plate (A) and attached to two manipulators at shoulder and hip level (B). Both manipulators are adjustable in height and driven by an electromotor, pushing and pulling the subject. Body kinematics are measured by potentiometers attached to the rod (C). A safety harness is attached to the pyramidal construction (D) and an emergency button is mounted on the frame (E).
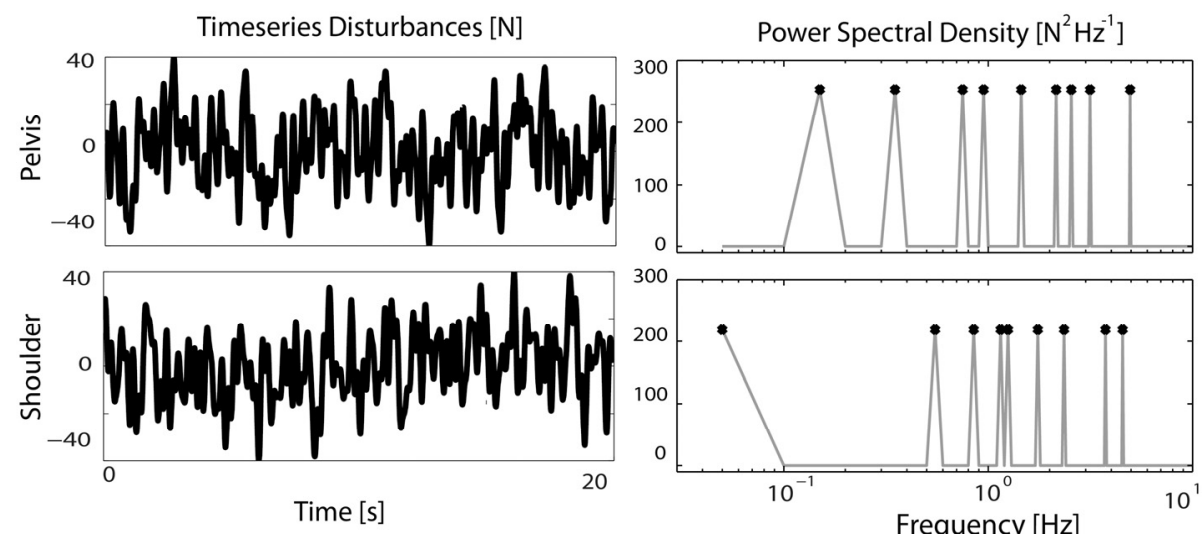

Figure 5.2: Time series of the applied force disturbances at the pelvis and between the shoulder blades, with the corresponding Power Spectral Density (PSD). The force disturbances are zippered multisine, as the excited frequencies (indicated with circles) are independent. 


\section{Procedures}

Participants were attached to the DIPP and stood with eyes open, without shoes, and their arms folded across their chest. Participants were instructed to maintain a normal upright stance position. Force disturbances at the hip and shoulder were applied simultaneously. External force fields were applied only at the level of the hip. The (spring) stiffness of the force field was set such that it compensated partially for the gravitational stiffness around the participant's ankle joint, and was normalized to $\mathrm{mgl}$ (in which $\mathrm{m}$ is the mass of the participant, $g$ the gravitational acceleration and $l$ the height of the CoM (Center of Mass), estimated by $0.575^{*}$ length of the participant).

Within the five experimental trials, a fixed stiffness level was set, expressed in a percentage of full compensation. One baseline trial was recorded without force field (0\%). Three force fields were stabilizing the human body $(20 \%, 50 \%$, and $80 \%)$, one force field destabilized the human body $(-20 \%)$. All trials were randomized, and participants were allowed to rest between trials according to their needs. Prior to the experiments, participants were allowed to get familiarized with the disturbances according to their individual needs. In case of the five force fields trials, participants were allowed to familiarize approximately 10 seconds before recording the data. This excluded any transient effects in the responses.

\section{Recordings}

Body kinematics were measured using two draw-wire potentiometers (Celesto SP2-25, Celesto, Chatsworth, CA, United States), which were attached to the end of the push/pull rods and measured the displacement of the participant's upper and lower body segments. A dual-force plate (AMTI; Watertown, USA), measured the ground reaction forces and torques in six degrees of freedom under each foot. All signals were recorded with a sample frequency of $1000 \mathrm{~Hz}$ and processed in Matlab (The MathWorks, Natick, MA, United States).

\section{Data analysis}

To study the age-related changes in adaptation of multi-joint coordination, different dataanalysis methods were used. In various studies (Horak and Nashner, 1986; Creath et al., 2005), multi-joint postural control is described by the ankle and hip strategy, which are defined as movements around the ankle and hip joints. Therefore, sway and torque responses of young and elderly participants were compared, expressed by the Root Mean Square (RMS). The RMS gives an indication of the effective amplitude, instead of a maximum, yielding a representative value for the response amplitudes. Secondly, covariance descriptors (Kuo et al., 1998) resulted from a kinematic analysis, which included postural coordination between the ankle and hip joints. To include multi-segmental influences in postural control (Boonstra et al., 2013), system identification techniques were used, which resulted in Frequency Response Functions (FRFs). The FRFs describe the relationship between the torques and the angles as a response to the disturbances only, i.e. intersegmental coordination of the neuromuscular controller is identified. Finally, to find physiologically relevant parameters that describe the underlying mechanisms of multi-joint 
coordination, a model was fit on the FRF. This resulted in an estimate of time delays and parameters describing intrinsic and reflexive feedback properties.

\section{Preprocessing}

All signals were filtered with a phase preserving fourth-order Butterworth filter, with a cutoff frequency of $10 \mathrm{~Hz}$. The ankle and hip angle were calculated with goniometric rules from the recorded upper and lower body displacements and the participant's dimensions. The hip angle was described as the angle of the upper body with respect to the longitudinal axis of the upper leg. From the force plate data, the total ankle and hip torques were calculated with inverse dynamics (Winter, 1990; Schwab, 1998). Data were segmented in 20 s periods of the disturbance signals, yielding nine data blocks per trial. From each data block, the mean and trends were removed. The responses were averaged over the nine data blocks for each condition and participant and used for further analysis.

\section{Root Mean Square and Covariance descriptor}

A description of the response amplitude of the ankle and hip angle and the ankle and hip torque was given by the Root Mean Square (RMS).

To represent the upper and lower body movement and their coupling, the two-by-two covariance matrix of the ankle and hip angle was used (Kuo et al., 1998).

$$
Q=\operatorname{cov}(\theta)=\frac{1}{n-1} \sum_{i=1}^{n}\left(\theta_{i}-\bar{\theta}\right)\left(\theta_{i}-\bar{\theta}\right)^{T}
$$

In which $\theta_{i}$ is the $\mathrm{i}^{\text {th }}$ time sample of the vector containing the ankle and hip angles. The diagonal terms of the covariance matrix represent the variances of the ankle and hip angle. The off-diagonal terms represent the inter-segmental coupling between the ankle and hip.

The covariance matrix may be described by an ellipse (Kuo et al., 1998; Speers et al., 2002). The extent of the ellipse along the horizontal and vertical axes is proportional to the RMS motion of the ankle and hip, respectively. The eigenvalues of the covariance matrix, $\lambda_{1}$ and $\lambda_{2}$, describe the squared lengths of the major and minor ellipse axes, respectively. The long axis of the ellipse $\left(\lambda_{1}\right)$ represents the amount of hip strategy utilized by the participant, while the minor axis of the ellipse $\left(\lambda_{2}\right)$ is an approximate indicator of the amount of CoM movement. The eigenvector with the largest eigenvalue was used to calculate the orientation angle $(\alpha)$ of the ellipse. The angle quantifies the direction of the relationship between the ankle and hip angles.

\section{Frequency response functions}

The dynamical properties of the neuromuscular controller were quantified in the frequency domain by Frequency Response Function (FRFs). The FRFs consist of two parts: a magnitude and a phase, describing the relationship between disturbances and the responses in terms of magnitude and time, respectively. Using closed-loop system identification 
techniques (Engelhart et al., in press; van der Kooij et al., 2005), the FRFs of the neuromuscular controller $\left(H_{c}\right)$ are computed from the experimental data, according to:

$$
H_{c}=-S_{d T}\left(S_{d \theta}\right)^{-1}
$$

In which $S_{d T}$ and $S_{d \theta}$ are the cross-spectral density matrices between the external disturbances (d) to the corrective ankle and hip torques $(T)$ and the ankle and hip angles $(\theta)$, respectively, resulting in a two-by-two FRF matrix $\left(H_{c}\right)$. Inverting the $S_{d \theta}$ matrix and multiplying it with the $S_{d T}$ matrix requires that the matrix components are known at all excited frequencies, which is untrue for the zippered multisine, as both disturbance signals contain different frequencies. Therefore, the complex numbers of the cross-spectral densities were interpolated in terms of magnitude and phase, to obtain all matrix components for the full range of excited frequencies in the zippered multisine. The FRFs were only evaluated at the frequencies where the disturbance signals contained power. The FRFs were normalized by the mass and length of the participants to compensate for differences in the participants' mass and pendulum length, which influences the FRFs.

$H_{c}$ consists of two direct terms, covering the FRFs from ankle angle to ankle torque $\left(H_{c, \theta_{a n k} 2 T_{a n k}}\right)$ and from hip angle to hip torque $\left(H_{c, \theta_{\text {hip }} 2 T_{\text {hip }}}\right)$. These direct terms quantify the ankle and hip contributions to balance control. Furthermore, there are two indirect terms, which cover the FRFs from ankle angle to hip torque $\left(H_{c, \theta_{a n k} 2 T_{\text {hip }}}\right)$ and from hip angle to ankle torque $\left(H_{c, \theta_{\text {hip } 2 T_{\text {ank }}}}\right)$ and reflect the intersegmental coupling (Boonstra et al., 2013).

\section{Model description}

A FRF describes the behaviour of the system, but it does not reveal which physiological mechanisms are underlying the system. To relate the changes in behaviour to the changes in the underlying physiology, a model of the neuromuscular controller was fitted on the FRFs (Figure 5.3).

The neuromuscular controller stabilizes the human body by generation of joint torques. The corrective joint torques around the ankles and hips result from intrinsic feedback together with delayed neural feedback. Each system in the neuromuscular controller was described by a mathematical formula (i.e. transfer function) with parameters describing the physiology. This resulted in a model for the four terms of the neuromuscular controller:

$$
\begin{gathered}
H_{c, \theta_{\text {ank }} 2 T_{\text {ank }}}=H_{p}^{\text {ank }}+H_{r}^{\text {ank } 2 T_{\text {ank }}} H_{T D}^{\text {ank }} \\
H_{c, \theta_{\text {hip }} 2 T_{\text {ank }}}=H_{r}^{\text {hip } 2 T_{\text {ank }}} H_{T D}^{\text {ankhip }} \\
H_{c, \theta_{\text {ank }} 2 T_{\text {hip }}}=H_{r}^{\text {ank2} 2 T_{\text {hip }}} H_{T D}^{\text {ankip }} \\
H_{c, \theta_{\text {hip }} 2 T_{\text {hip }}}=H_{p}^{\text {hip }}+H_{r}^{\text {hip } 2 T_{\text {hip }}} H_{T D}^{\text {hip }}
\end{gathered}
$$


The transfer function of intrinsic feedback $\left(H_{p}\right)$ describes the muscle and tendon dynamics together with the soft tissue properties. Intrinsic feedback is described for each joint by only stiffness $\left(H_{p}=K_{p}\right)$. Intrinsic dynamics only exists directly at joint level, i.e. the direct terms of the neuromuscular controller, with different values for the ankle and hips $\left(H_{p}^{a n k}, H_{p}^{\text {hip }}\right)$.

In the transfer function of the lumped time delay $\left(H_{T D}=e^{-\tau_{d} s}\right), \tau_{d}$ represents the sum of neural conduction time (transport delay), an electromechanical delay (to activate the muscles), and the processing time of sensory information. A separate lumped delay was introduced for each path length the sensory information travels, e.g. the direct terms of the neuromuscular controller had separate delays $\left(H_{T D}^{a n k}, H_{T D}^{\text {hip }}\right)$ and the indirect terms of the FRF had equal delays $\left(H_{T D}^{\text {ankhip }}\right)$.

The transfer function of reflexive feedback $\left(H_{r}\right)$ was represented by a matrix with stiffness and damping terms, relating the joint torques to the ankle and hip angles and angular velocities. This resulted in four transfer functions.

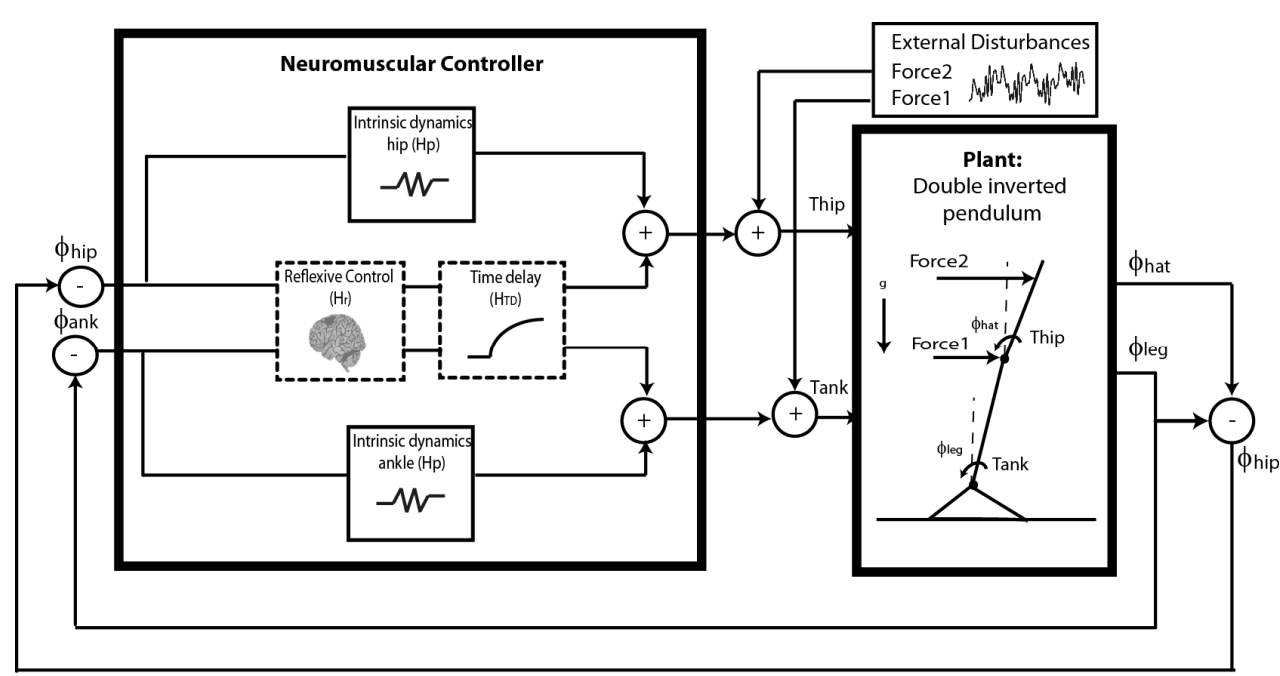

Figure 5.3: Schematics of the human balance control system. The human body is represented as a double inverted pendulum, with disturbances acting at the hip and shoulder level. The model of the neuromuscular controller was used for parameter estimation. The inputs are the leg and hip angle $\left(\theta_{\text {ank }}, \theta_{\text {hip }}\right)$ and the outputs the corrective joint torques $\left(T_{\text {ank }}, T_{\text {hip }}\right)$. Intrinsic dynamics were modelled as a spring, and were different for the ankle and hip joints. Reflexive control and time delay dynamics were MIMO transfer functions (shown as dotted boxes), in which interaction existed between the ankle and hip joint signals. 


$$
\begin{aligned}
& H_{r}^{a n k 2 T_{\text {ank }}}=K_{\text {ank2T } T_{\text {ank }}}+D_{\text {ank } 2 T_{\text {ank }}} S \\
& H_{r}^{\text {hip } 2 T_{\text {ank }}}=K_{\text {hip } 2 T_{\text {ank }}}+D_{\text {hip } 2 T_{\text {ank }}} S \\
& H_{r}^{\text {ank2T }}{ }^{\text {hip }}=K_{\text {ank2T }} T_{\text {hip }}+D_{\text {ank } 2 T_{\text {hip }}} s \\
& H_{r}^{\text {hip } 2 T_{\text {hip }}}=K_{\text {hip } 2 T_{\text {hip }}}+D_{\text {hip } 2 T_{\text {hip }}} S
\end{aligned}
$$

The model had a total of 13 physiologically interpretable parameters. Table 5.2 shows the model parameters and how they are estimated, as is outlined next.

\section{Model fitting}

The transfer functions from the model were fitted onto the experimental FRFs using a nonlinear least-squares optimization algorithm. The algorithm searches for a parameter set that minimizes the objective function $\left(J_{i, f_{k}}\right)$.

$$
\begin{gathered}
J_{i, f_{k}}=\sqrt{\frac{1}{1+f_{k}}}\left|\ln \left(H_{c, i}\left(f_{k}, p\right)\right)-\ln \left(\widehat{H}_{c, i}\left(f_{k}\right)\right)\right| \\
S S E=\sum_{i=1}^{4} \sum_{k=1}^{18} J_{i, f_{k}}
\end{gathered}
$$

The logarithmic difference between the FRF $\left(H_{c}\right)$ based on the calculated parameter vector $(p)$ and the estimated $\operatorname{FRF}\left(\widehat{H}_{c}\right)$ obtained from experimental data was summed over the frequencies $\left(f_{k}, k=1: 18\right)$ and the different terms of the FRFs $\left(H_{i}, i=1: 4\right)$, resulting in an SSE (sum squared error). The objective function is chosen such that there is more emphasis on the low frequencies $\left(\frac{1}{1+f_{k}}\right)$, where stiffness typically manifests. In addition, a relative error is calculated for all frequencies on a logarithmic scale.

The optimization algorithm was run 20 times with random initial conditions to assure a global minimum was found. The best parameter set was obtained from the fit with the lowest SSE value. In addition, the goodness of fit (GOF) describes how well the data compare to the estimation with the parameter set and is expressed as:

$$
G O F_{i}=1-\frac{\sum\left|H_{c, i}\left(f_{k}, p\right)-\widehat{H}_{c, i}\left(f_{k}\right)\right|^{2}}{\sum\left|\widehat{H}_{c, i}\left(f_{k}\right)\right|^{2}} * 100 \%
$$

The models were fitted for each participant on all five experimental trials simultaneously. To limit the number of parameter combinations in the fit, lumped time delays and intrinsic feedback were constant over trials in which force field levels were varied. The lumped time delays of the indirect terms were calculated as the averages of the direct terms. Reflexive feedback was variable over trials as it was assumed that subjects alter their control action when they are exposed to a force field. 
Table 5.2: Overview of the parameters as used in the model fits. The parameters were fit to the five force field conditions simultaneously and for each subject individually. Time delay and intrinsic feedback parameters were kept constant over the five conditions, while the values for the reflexive feedback parameters varied over the conditions. The time delay for ankle-hip and vice versa, were calculated as the average of the time delay ankle and time delay hip.

\begin{tabular}{|c|c|c|c|}
\hline & Parameter & Unit & Varied over conditions \\
\hline \multicolumn{4}{|l|}{ Time delay } \\
\hline Ankle & $\tau_{d}^{a n k}$ & s & No \\
\hline Hip & $\tau_{d}^{h i p}$ & s & No \\
\hline Ankle-Hip and vice versa & $\tau_{d}^{\text {ankhip }}$ & s & - \\
\hline \multicolumn{4}{|l|}{ Intrinsic feedback } \\
\hline Stiffness ankle & $K_{p}^{a n k}$ & $\mathrm{Nm} / \mathrm{rad}$ & No \\
\hline Stiffness hip & $K_{p}^{\text {hip }}$ & $\mathrm{Nm} / \mathrm{rad}$ & No \\
\hline \multicolumn{4}{|l|}{ Reflexive feedback } \\
\hline Stiffness Ank2Tank & $K_{\text {ank2T ank }}$ & $\mathrm{Nm} / \mathrm{rad}$ & Yes \\
\hline Stiffness Hip2Tank & $K_{\text {hip } 2 T_{a n k}}$ & $\mathrm{Nm} / \mathrm{rad}$ & Yes \\
\hline Stiffness Ank2Thip & $K_{\text {ank2Thip }}$ & $\mathrm{Nm} / \mathrm{rad}$ & Yes \\
\hline Stiffness Hip2Thip & $K_{\text {hip } 2 T_{\text {hip }}}$ & $\mathrm{Nm} / \mathrm{rad}$ & Yes \\
\hline Damping Ank2Tank & $D_{\text {ank2T }}$ ank & $\mathrm{Nms} / \mathrm{rad}$ & Yes \\
\hline Damping Hip2Tank & $D_{\text {hip2T }}$ ank & $\mathrm{Nms} / \mathrm{rad}$ & Yes \\
\hline Damping Ank2Thip & $D_{\text {ank2Thip }}$ & $\mathrm{Nms} / \mathrm{rad}$ & Yes \\
\hline Damping Hip2Thip & $D_{\text {hip2T hip }}$ & $\mathrm{Nms} / \mathrm{rad}$ & Yes \\
\hline
\end{tabular}

\section{Statistical analysis}

The characteristics of the participants were represented by mean and standard deviation in case of a Gaussian distribution. Else, median, and inter quartile range or number and percentage were presented. To test significant differences between groups, an independent two-sided t-test was performed. In case of the non-normal distributed values, a MannWhitney $U$ test was performed.

To test significant differences in RMS and covariance descriptors between age and force field levels, linear mixed models were used. Age and force field level were fixed effects. To account for the repeated measurements, participant intercept was included as a random effect. In addition, interaction effects between age and force field levels were studied.

For statistical analysis of the FRFs, the magnitude of each FRF was logarithmically transformed to make the data normally distributed. Subsequently the magnitudes were averaged within three frequency bands ( $<1 \mathrm{~Hz}, 1-2.5 \mathrm{~Hz}$ and 2.6-5 Hz). The lowest frequencies generally describe the stiffness properties of the system, whereas the magnitude at middle and high frequencies is shaped by damping and mass (inertia), respectively. Linear mixed models were used to test significant differences in FRFs between 
age and force field levels for each frequency band. Age group, force field level, and frequency band were fixed effects and participant intercept was a random effect. In addition, interaction effects between age group and force field level were studied.

To test significant differences of the estimated model parameters that were constant over trials, an independent two-sided t-test was performed, as there can only be an effect of age and not of force field level. To test significant differences between age and different force field levels in the estimated model parameters that were allowed to vary over trials, linear mixed models were used. Age group and force field level were fixed effects, and participant intercept was a random effect. In addition, interaction effects between age and force field level were studied.

For all tests, the significance level (a) was set at 0.05. All analyses were performed with SPSS version 22.0 (SPSS, Chicago, IL).

\subsection{Results}

Characteristics of the group of healthy young and healthy old participants are presented in Table 5.1 to illustrate the health of the study population The elderly used more medication $(p=0.001)$, had lower MMSE score $(p=0.023)$, and lower handgrip strength $(p=0.014)$ compared with the young participants. All subjects were able to maintain balance during the disturbances and the force field trials.

\section{Root mean Square}

Figure 5.4 shows the joint angles and torques of a representative young and elderly participant, in case only the force disturbances were applied without an external force field. Figure 5.5 shows the RMS of the ankle and hip angle and the ankle and hip torque. The elderly had smaller ankle $(p=0.001)$ and hip $(p=0.003)$ angles than the young participants. The exerted ankle and hip torques were not found to be significantly different between the two groups.

For every $10 \%$ increase in external force field, the postural responses decreased, namely the ankle angle with $-0.02 \mathrm{deg}(\mathrm{p}<0.001)$, the hip angle with $-0.03 \mathrm{deg}(\mathrm{p}<0.001)$, the ankle torque with $-0.7 \mathrm{Nm}(p<0.001)$ and the hip torque with $-0.07 \mathrm{Nm}(p<0.001)$.

An interaction effect was found between age and force field level; with increasing force field level, the decrease in ankle $(p=0.007)$ and hip $(p=0.004)$ angle was less in the elderly than the observed decrease in young participants. No interaction effect was found between age and force field level for the ankle and hip torque. The elderly adapted their torques to different force field levels compared to the young participants. 

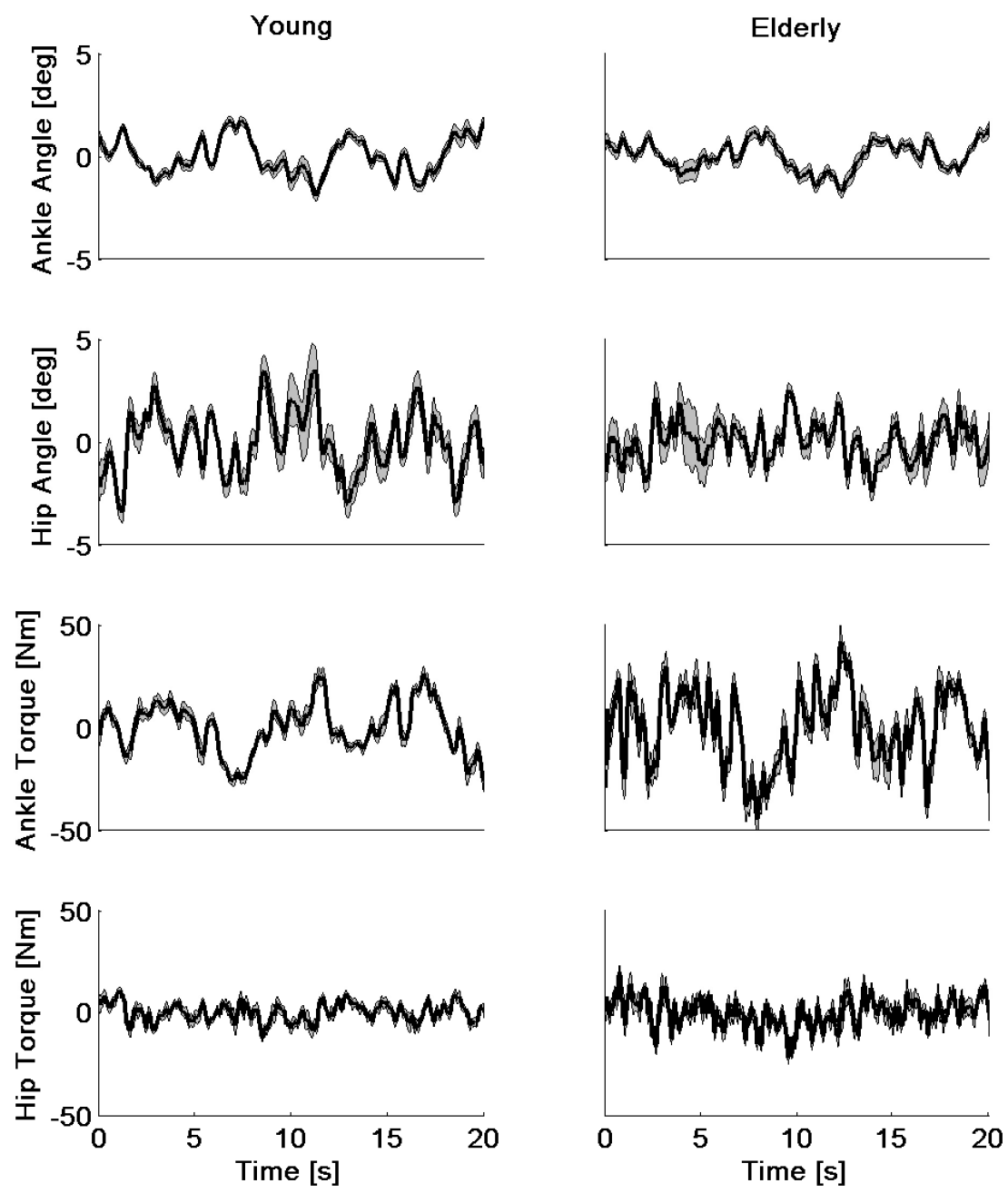

Figure 5.4: Joint angles and joint torques in response to the applied disturbances of a representative young (left) and elderly (right) participant in the baseline trial without force field (0\%). The average over the nine disturbance cycles is indicated with the black line; the grey area represents the standard deviation. 

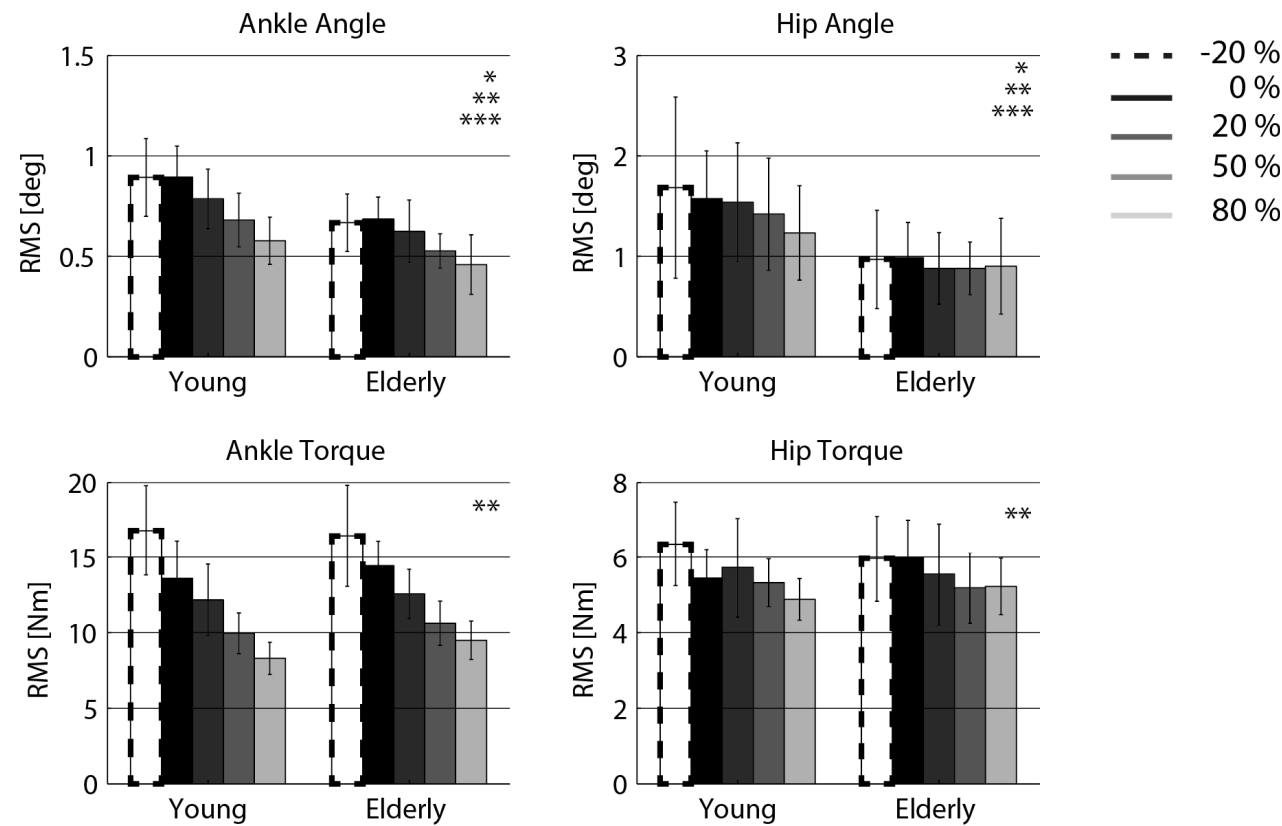

Figure 5.5: Root Mean Square (RMS) values of the joint angles and torques, for young and elderly participants per force field level represented by mean and standard deviation. The asterisks represent significant differences with age $(*)$, force field $(* *)$ or the interaction between age and force field $(* * *)$.
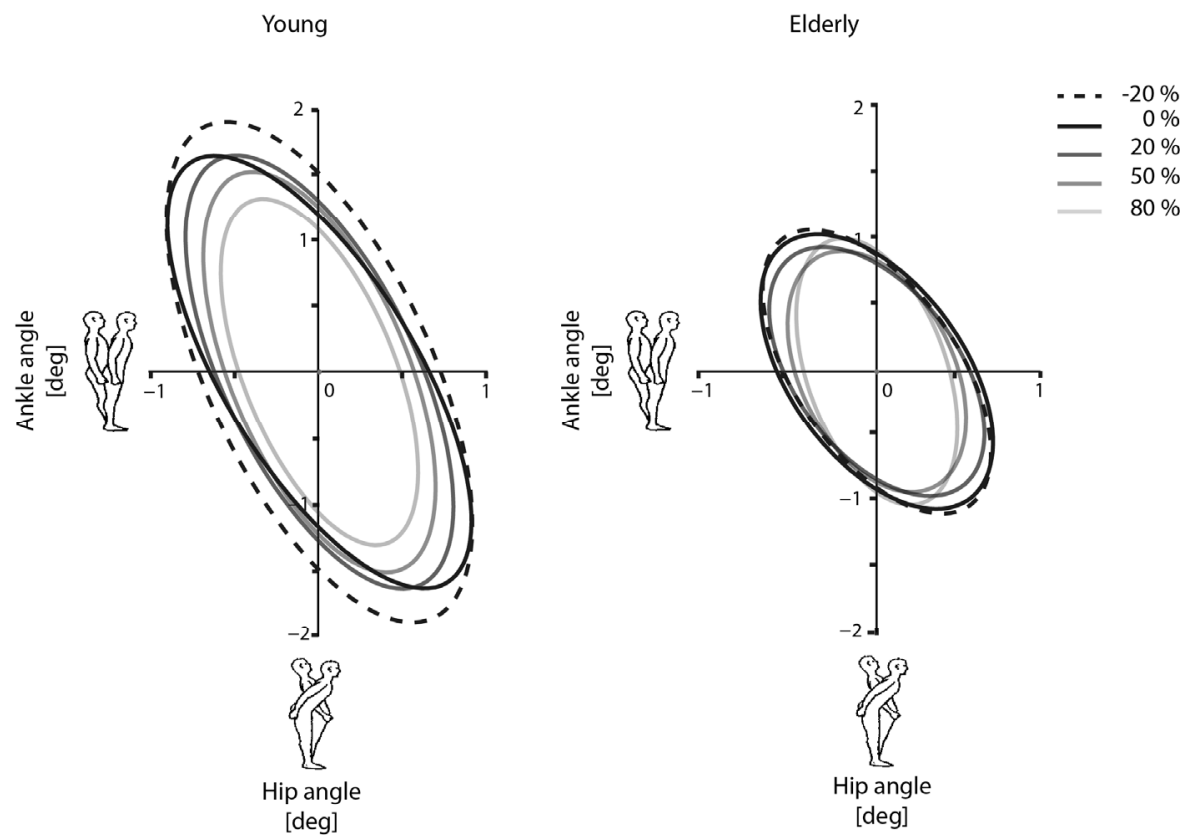

Figure 5.6: Covariance descriptor of the young and elderly participants per force field level. 


\section{Covariance Descriptor}

Figure 5.6 shows the covariance descriptor for the young and elderly participants for the different force field levels. The length of the ellipse major axis was lower in the elderly compared to the young participants $(p=0.013)$. No differences were found for the minor axis or the orientation of the ellipse between the young and elderly.

When applying a stiffer force field, the orientation of the ellipse increased and the covariance descriptors decreased. For every $10 \%$ increase in force field, the values altered; $\lambda_{1}(-0.013, \mathrm{p}<0.001), \lambda_{2}(-0.001, \mathrm{p}<0.001)$ and $\alpha(0.002 \mathrm{rad}, \mathrm{p}<0.001)$.

An interaction effect between age and force field level was found; the decrease of $\lambda_{1}$ was less in the elderly compared to the young $(p=0.01)$. No interaction effect was found between age and force field level for the $\lambda_{2}$ and $\alpha$; the elderly adjusted these values compared to the young participants.

\section{Frequency Response Functions}

Figure 5.7 shows the neuromuscular controller FRF $\left(\widehat{H}_{c}\right)$ of the young and elderly in the baseline trial, when only disturbances were applied without external stiffness (0\%).

There was a main effect of age, as the magnitude of $H_{c, \theta_{a n k} 2 T_{a n k}}$ was higher in the elderly for the lowest $(p=0.002)$ and midrange $(p<0.001)$ frequencies. For the other terms, the magnitude was higher in the elderly in the midrange frequencies of $H_{c, \theta_{\text {hip }} 2 T_{\text {ank }}}(p=0.001)$ and $H_{c, \theta_{a n k} 2 T_{\text {hip }}}(\mathrm{p}=0.006)$. No significant differences with age were found in any of the frequency bands for $H_{c, \theta_{h i p} 2 T_{h i p}}$ and no differences were found for the high-frequency range of the neuromuscular controller.

When applying a force field, the FRF magnitude changed (Figure 5.8). With increasing force field, the FRF magnitudes became lower for the entire frequency range in $H_{c, \theta_{a n k} 2 T_{a n k}}$ ( $\mathrm{p}<0.001$, for all frequency bands) and $H_{c, \theta_{\text {hip }} 2 T_{\text {ank }}}(\mathrm{p}<0.001$, for all frequency bands). A significant decrease of FRF magnitude was also found for the lowest and midrange frequencies in $H_{c, \theta_{a n k} 2 T_{h i p}}(\mathrm{p}<0.005$, for both frequency bands) and the midrange frequencies of $H_{c, \theta_{\text {hip }} 2 T_{\text {hip }}}(\mathrm{p}<0.016)$.

An interaction effect between age and force field level was found for the low and midrange frequencies in $H_{c, \theta_{\text {ank }} 2 T_{\text {ank }}}(\mathrm{p}=0.027$ and $\mathrm{p}=0.008)$ and $H_{c, \theta_{\text {hip }} 2 T_{\text {ank }}}(\mathrm{p}=0.026$ and $\mathrm{p}=0.002)$. Elderly participants reduced the FRF magnitude around the ankle less compared to young participants. The FRF magnitude around the hips was adjusted comparable in both groups. For example, the magnitude of $H_{c, \theta_{a n k} 2 T_{a n k}}$ for the lowest frequencies in young participants was decreased by $21 \%$ for the stiffest force field $(80 \%)$, whereas for the elderly it was decreased by $14 \%$. For the destabilizing force field $(-20 \%)$, postural responses increased $28 \%$ in young, whereas for the elderly they were only increased by $8 \%$. 

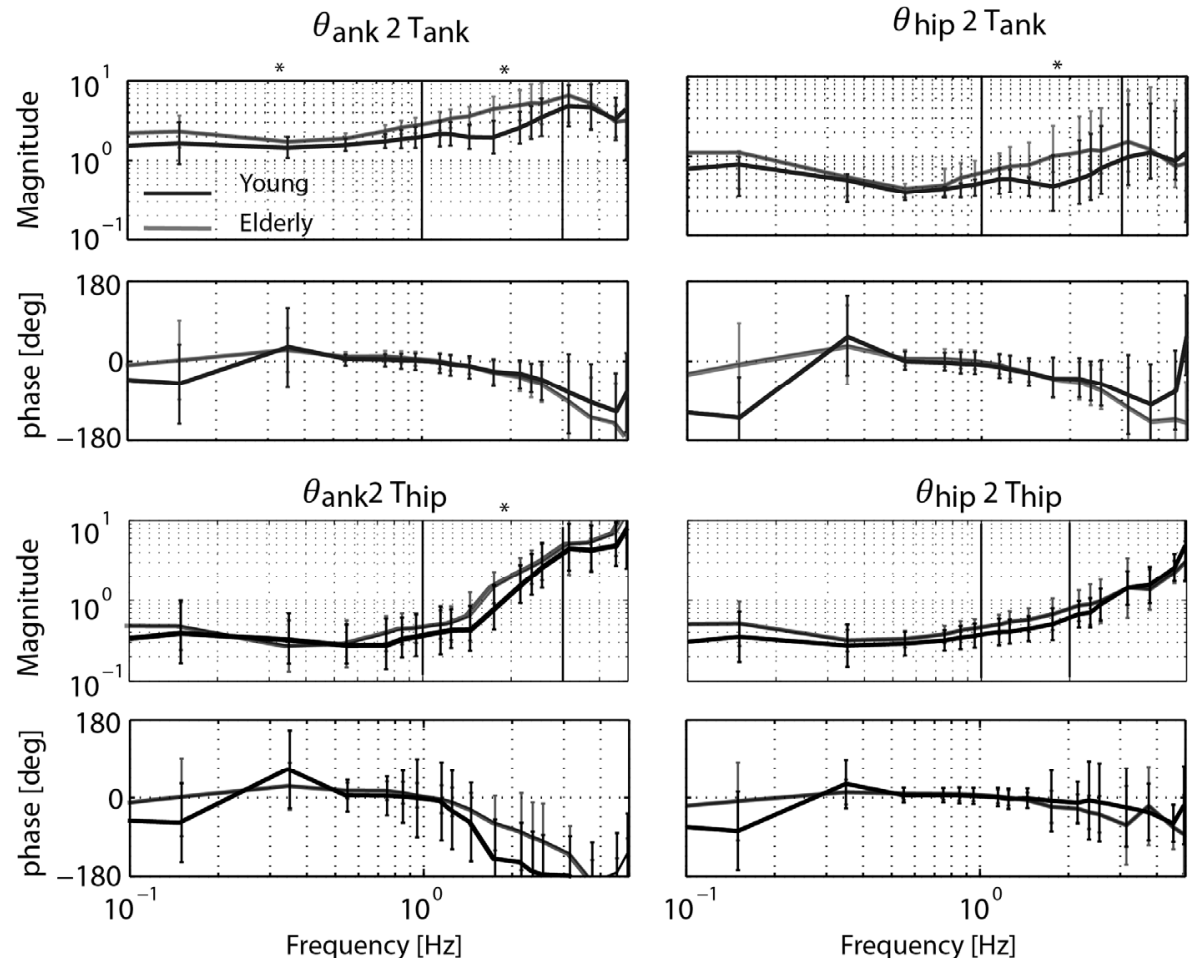

Figure 5.7: Baseline differences in Frequency Response Function (FRF) between young (black) and elderly (grey) participants, where only the two disturbances were applied, without additional force fields $(0 \%)$, represented by mean and standard deviation. The FRF consists of four terms, two direct terms from ankle angle to ankle torque $\left(\theta_{\text {ank }} 2 T_{\text {ank }}\right)$ and from hip angle to hip torque $\left(\theta_{\text {hip }} 2 T_{\text {hip }}\right)$, and two indirect terms from hip angle to ankle torque $\left(\theta_{\text {hip }} 2 T_{\text {ank }}\right)$, and from ankle angle to hip torque $\left(\theta_{\text {ank }} 2 T_{\text {hip }}\right)$. The asterisk represent the frequency bins in which there is a significant difference with age $(*)$. 


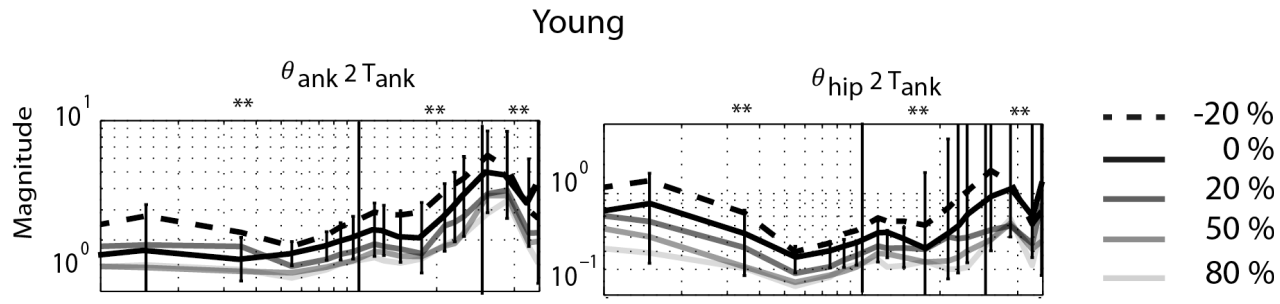

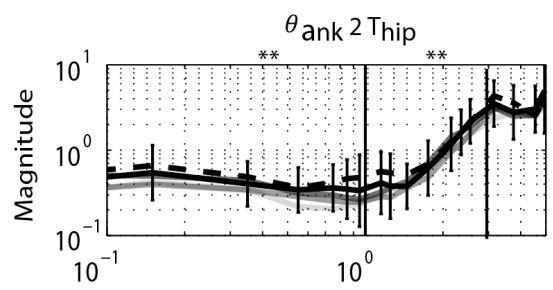

Frequency [Hz]

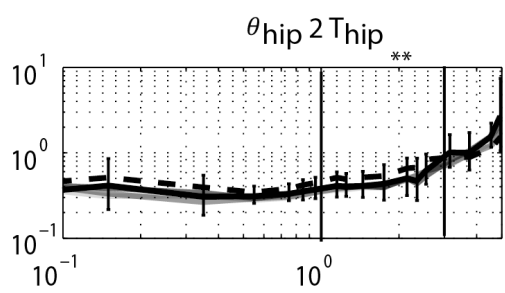

Frequency [Hz]

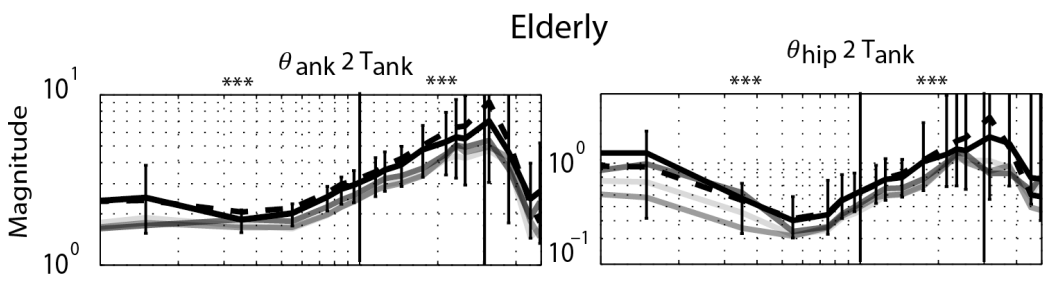

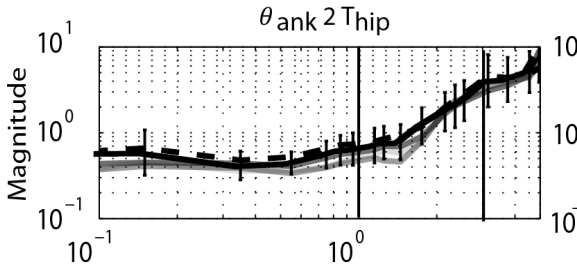

Frequency [Hz]

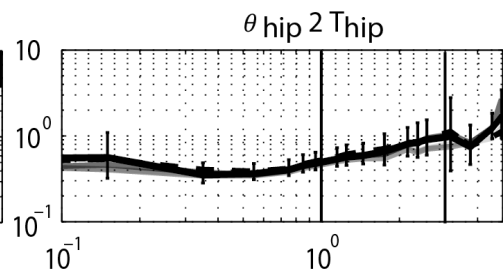

Frequency [Hz]

Figure 5.8: Adaptation of Frequency Response Function (FRF) magnitude in the young (upper part) and elderly (lower part) participants. For each force field level the mean is shown. For displaying reasons only the standard deviation was shown for the baseline trial. Standard deviations of the other force field levels were comparable to the baseline trials. The asterisks represent the frequency bins in which there are significant differences with force field $\left({ }^{* *}\right)$ or the interaction between age and force field $(* * *)$. 


\section{Model parameters}

The average GOF (and standard deviation) over force field levels for each FRF term $\left(H_{c, \theta_{a n k} 2 T_{a n k}}, H_{c, \theta_{h i p} 2 T_{a n k}} H_{c, \theta_{a n k} 2 T_{h i p}}\right.$ and $\left.H_{c, \theta_{h i p} 2 T_{h i p}}\right)$ was for the young $(81(14) \%, 70$ $(18) \%, 50(23) \%$ and $75(17) \%)$ and for the elderly (75 (13)\%, $70(13) \%, 63(20) \%$ and $82(14) \%)$. Similar GOF values were obtained for the young and elderly. Different GOF values were found between conditions, e.g. some of the conditions had higher GOF values, and thereby were fit better than others. Furthermore, the GOF values of the direct FRF terms were higher than those of the indirect terms and the GOF values of $H_{c, \theta_{a n k} 2 T_{h i p}}$ was lowest. The goodness of fit is also displayed in Figure 5.9, which shows the estimated FRFs together with the model fit for a representative participant.

Figure 5.10 shows the estimated parameters for the young and elderly participants. The elderly had larger lumped time delays for the hips $(p<0.001)$ compared to the young participants. The lumped time delays of the ankle were not found to be significantly different between age groups. Furthermore, the intrinsic ankle stiffness $(p=0.007)$ was higher in elderly, but no significant differences were found for the intrinsic hip stiffness. None of the reflexive stiffness and damping terms were found significantly different between young and elderly.

When increasing the force field level in both groups, all reflex stiffness and damping values decreased $(p<0.04)$. An interaction effect was found between age and force field level only for $K_{a n k 2 T_{a n k}}(\mathrm{p}=0.04)$. The elderly reduced their reflexive stiffness around the ankle joint less for increasing force fields compared to the young.
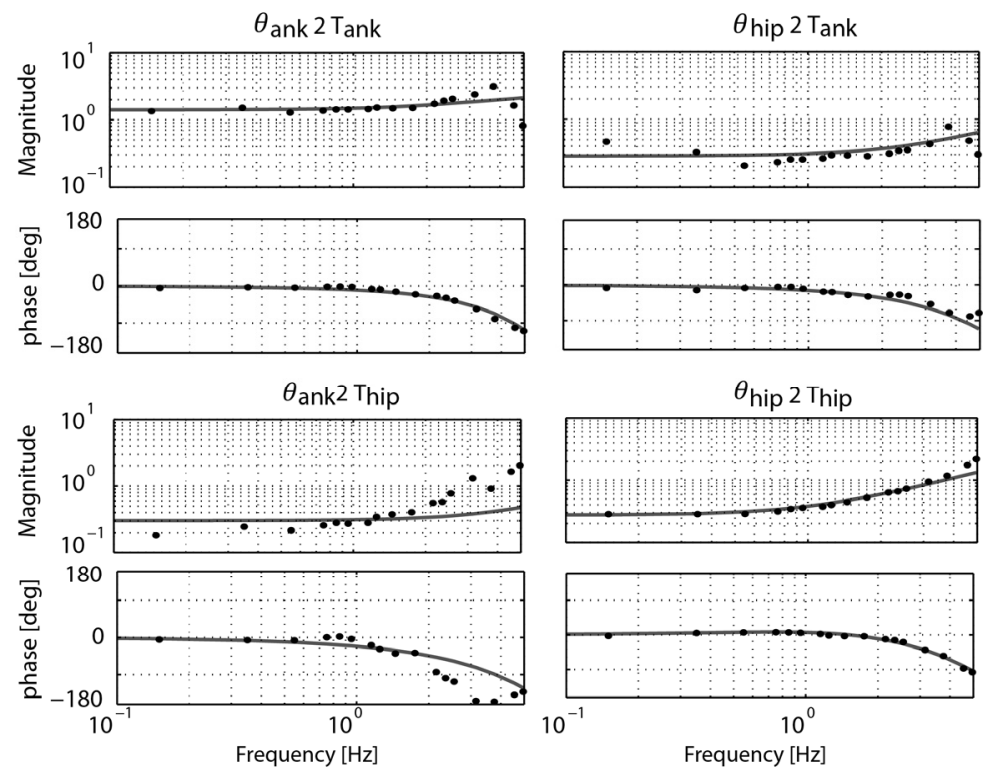

Figure 5.9: Frequency Response Functions (FRFs) based on measured data (black dots) and model fits (grey line) of a representative healthy young subject for the baseline trial $(0 \%)$. 

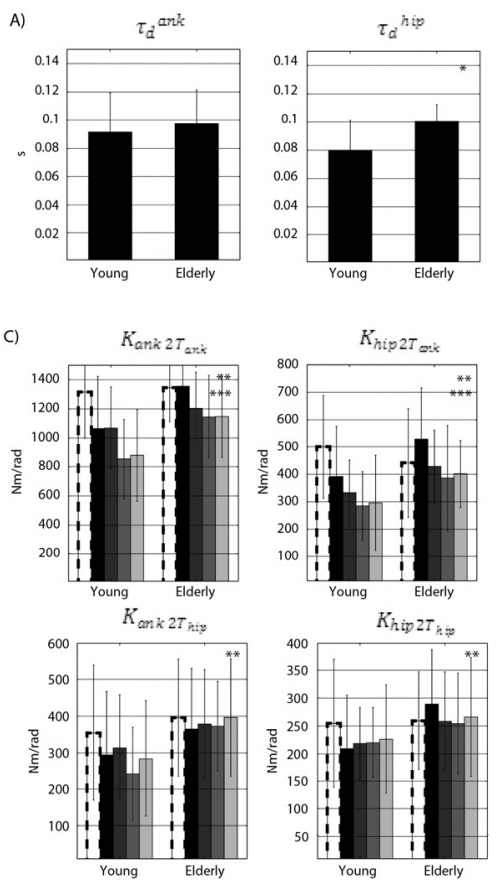
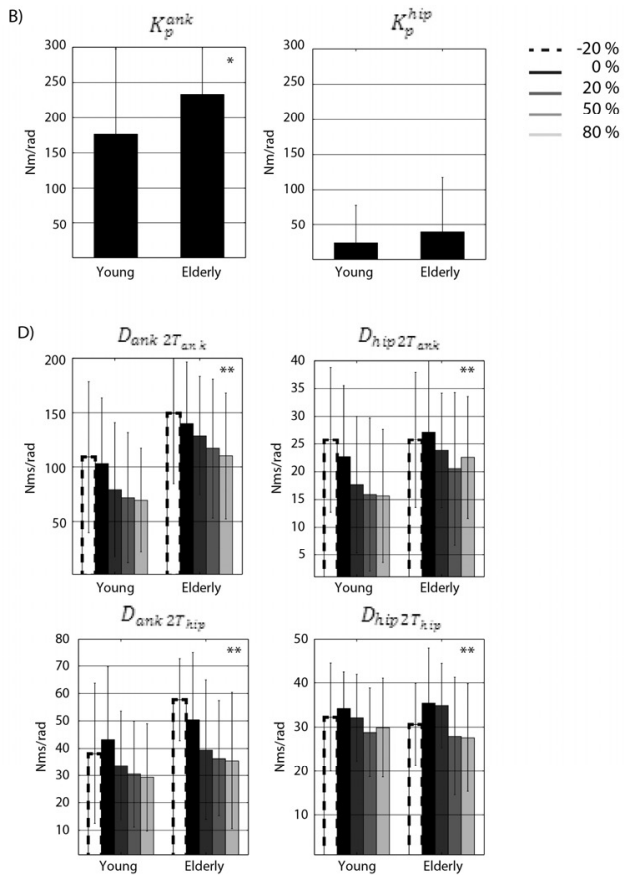

Figure 5.10: Estimated parameters represented by mean and standard deviation (error bars), for young and old participants per force field level. Panel A) shows the estimated time delays, B) the intrinsic properties, C) the reflexive stiffness and D) the reflexive damping. The asterisks represent significant differences with age $(*)$, force field $\left({ }^{* *}\right)$ or the interaction between age and force field $(* * *)$.

\subsection{Discussion}

The results of this study show age-related differences in multi-joint coordination. The elderly swayed less than the young participants, and the elderly showed a reduced hip strategy. As the corrective joint torques were not significantly different between age groups, the FRF magnitude was higher in the elderly. The relationship between the corrective joint torques to compensate for the body sway was increased, i.e. the elderly exhibit a higher stiffness. Parameter estimation showed that the elderly have higher intrinsic stiffness around the ankle joint. When an external force field was applied, both age groups lowered their postural responses, expressed as lower FRF magnitude around the ankle. However, the elderly adapted their postural responses less compared to the young participants. Parameter estimation showed that adaptation was due to adjustment of their reflexes, both stiffness and damping, with level of force field. 


\section{Participant Characteristics}

Based on the inclusion criteria, all young and elderly participants were characterized as healthy. None of the young participants were taking medication. The elderly used medication, but none of the medication had a known influence on balance control. Comparison between young and elderly participants showed a significant lower MMSE score in the elderly. It is known that cognitive processing has an influence on the control of balance (Teasdale and Simoneau, 2001; Doumas et al., 2009; Ambrose et al., 2013; Stijntjes et al., 2015), e.g. low cognitive function increases the risk of imbalance. Although all of the elderly were characterized as healthy with normal cognitive function, the lower MMSE score might have influenced the results. None of the participants reported fear of falling or fatigue during the experiment.

\section{Root Mean Square and Covariance Descriptor}

Results show that the elderly swayed less when being disturbed by forces at the hip and shoulder, compared to the young participants. These results were in contrast with multiple studies of quiet stance, showing that body sway increased with age (Abrahamová and Hlavačka, 2008; Demura et al., 2008; Pasma et al., 2014a). In our study, the balance control system was externally disturbed. Humans altered their feedback gains to correct for these disturbances, i.e. the amount of joint torque relative to the amount of joint motion was adjusted. To be more specific, with increasing force field level, the feedback gains were reduced. In quiet stance, the balance control system is mainly influenced by internal disturbances, such as sensory and motor noise, which cannot be corrected for. Altering feedback gains during quiet stance would amplify the internal disturbances, which increases body sway (Speers et al., 2002). This explains why in quiet stance, the elderly sway more and that it is possible that in perturbed stance the elderly sway less.

Covariance descriptors were used to describe not only whether the application of force fields resulted in changes of sway, but also in postural coordination. Eigenvectors and eigenvalues were used to describe independent combinations of joint movements, defined by principle component analysis (Kuo et al., 1998; Alexandrov et al., 2001a; Hsu et al., 2007). From the covariance matrix of the ankle and hip angle, the first component $\lambda_{1}$ may be interpreted as double-inverted pendulum behaviour and quantifies the amount of hip strategy. Our results show that $\lambda_{1}$ is smaller in the elderly, indicating they have less hip strategy and behave more like a single inverted pendulum. The second component $\lambda_{2}$ was not found to be significantly different between age groups, indicating the control of the CoM was similar. In a previous study (Hsu et al., 2013) where external disturbances (platform translations) were applied, the elderly exhibited a more rigid stance and a decreased variance between the joints was found, while the CoM excursion was not significantly different between the groups, which is in accordance with our findings.

When a stabilizing force field was applied, a decrease of both $\lambda_{1}$ and $\lambda_{2}$ was found as maintaining standing balance became easier. The combination of opposing ankle and hip motion was reduced, and a reduction was found in the combination of movement 
dominated more by the ankle. The negative relation remained; a positive ankle angle was accompanied with negative hip angle. There was an interaction effect between age and force field level. The elderly adapted their hip strategy less than young participants. This might be explained by the fact that the $\lambda_{1}$ was lower in elderly at baseline. The elderly adapted their control of CoM similar to the young.

\section{Frequency Response Functions}

In this study we used system identification techniques to solely identify the neuromuscular control mechanisms from the closed-loop feedback system, i.e. the corrective joint torques as a response to body movement around the joints. The neuromuscular controller dynamics and the coordination between joints has frequency specific effects, which were shown in FRFs. Compared to RMS values and covariance descriptors, the FRF is possibly a more sensitive measure. When analyzing the joint angles, it contains not only a sway response due to the disturbance, but the subject's own spontaneous sway (remnant sway due to e.g. motor and sensory noise) is also captured. RMS measures and covariance descriptors do not therefore distinguish between responses due to the disturbances and spontaneous sway. The FRF describes only the angles and corresponding corrective joint torques as a response to the disturbances, i.e. changes in the neuromuscular controller only.

In the elderly, the RMS values of the joint angles were lower and the joint torques were comparable to young adults. These results were also seen in higher FRF magnitude of elderly, at the lowest frequencies of the direct ankle term. Stiffness is assumed to dominate the magnitude of the FRFs in the low-frequency range, indicating that the elderly have higher ankle joint stiffness. When the pull of gravity is compensated for by an external force field, the elderly adapted their FRF magnitudes around the ankle less compared to young. These age-related differences in adaptation to force field levels were also found in the RMS outcomes and the covariance descriptors.

In addition to the adaptation at the lowest frequency range, also in the midrange frequencies significant differences were found between force field conditions. The midrange frequencies are believed to be most affected by damping properties. No significant differences were found between force fields conditions at high frequencies, which was consistent with our expectations. The FRF magnitude at high frequencies is generally shaped by the mass properties of the participants, which were not significantly different between young and elderly (Table 5.1 ).

\section{Model parameters}

Estimating model parameters on the FRF reveals the underlying mechanisms of the neuromuscular controller. The elderly exhibit a larger intrinsic stiffness of the ankle compared to the young, which is in concordance with our expectations following the results of the FRFs. Reflexive stiffness was not found to be significantly different between the age groups. When the force field level was increased, both the young and elderly reduced their reflexive stiffness and damping. However, the reflexive feedback gains around the ankle 
joint were reduced less in elderly compared to young participants. These results might indicate that the elderly were stiffer, but they were able to adapt their postural responses.

The body is mostly represented as an inverted pendulum, based on body rotation around the ankle joint. Our study includes a hip joint, which might result in different values of the estimated parameters compared to other studies. Kiemel et al. (Kiemel et al., 2008) estimated intrinsic joint parameters of both the ankles and hips using system identification techniques based on electromyography (EMG) signals and joint angles, when healthy young subjects were faced with visual scene disturbances. The intrinsic stiffness of the ankle and hips were found to be $293 \mathrm{Nm} / \mathrm{rad}$ and $95 \mathrm{Nm} / \mathrm{rad}$, respectively. Cenciarini et al. (Cenciarini, 2010) found intrinsic ankle stiffness of 157 and $99 \mathrm{Nm} / \mathrm{rad}$ for the young and elderly, respectively, when exposed to support surface tilts. The estimated stiffness values in this study are in the same range as the other two studies; however in our study the elderly exhibit larger intrinsic ankle stiffness compared to the young.

Estimated reflexive stiffness and damping of the ankle in the current study are within the ranges earlier found in the literature varying from 898 - $1500 \mathrm{Nm} / \mathrm{rad}$ and from $288-480$ Nms/rad (Peterka, 2002; Mahboobin et al., 2005; Cenciarini, 2010; Davidson et al., 2011). Those studies showed that the elderly exhibit higher reflex gains compared to young, which was consistent with our findings. Upper body stiffness and damping was found in healthy young subjects between 100-300 Nm/rad and 20-60 Nms/rad (Goodworth and Peterka, 2012). These values were similar to our results.

The time delays as estimated in this study consisted of processing time, electromechanical delay, and neural conduction time. Previous studies in which the human body is represented as an inverted pendulum, found a time delay of approximately $172 \mathrm{~ms}$, which was not significantly different between the young and elderly (Cenciarini, 2010). Other studies found time delays in the range of 100-200 ms (Peterka, 2002; Mahboobin, 2007; Davidson et al., 2011), and the elderly exhibited significantly higher delays, compared to the young (Davidson et al., 2011). This compares to our results.

\section{Clinical implications}

In this study we used system identification techniques to quantify age-related differences in adaptation of multi-joint coordination. With current clinical balance tests, the cause of altered balance control with age remains largely unknown (Visser et al., 2008; Pasma et al., 2014b) as it remains unclear if changes in balance control are due to a primary cause or an adaptive strategy. For example, if the elderly show more rigid behaviour, this can be due to increased intrinsic muscle stiffness or due to increased reflexive stiffness. With system identification techniques and parameter estimation, the contribution of the underlying mechanisms can be unraveled, as the balance control mechanism is expressed in physiologically relevant parameters (Engelhart et al., 2014). Our findings suggest that multijoint coordination is altered in the elderly, with increased intrinsic stiffness, which contributed to reduced adaptive capacities. Identification of the mechanisms that contribute 
to altered postural responses in the elderly provides insight into the factors influencing the risk of falling and can help to develop targeted interventions and reduce the risk of falling.

\section{Limitations of study}

With system identification techniques, we have derived parameters of the neuromuscular controller in multi-segmental balance control. Nevertheless, the results might be influenced by various factors. First, the control scheme of the neuromuscular controller may not be an adequate representation of actual postural control, and it may not describe all age-related changes in standing balance. We assumed that the states of the human body (joint angles and angular velocities) were fully known and the sensory information was "perfect". However, with age, the sensory systems might become impaired (Sturnieks et al., 2008). Furthermore, we did not model the integration of sensory information, i.e. the process of sensory reweighting (Peterka and Loughlin, 2004; Mahboobin et al., 2009). Therefore, agerelated changes in the quality of sensory information and sensory reweighting capacities were not studied.

Second, estimation of model parameters based on experimental data is a constant trade-off between a good model fit and the least amount of parameters. Increasing the number of parameters improves the fit; however the physiological interpretation becomes difficult. Two aspects are important when interpreting a parameter set obtained from a model fit on experimental data. The first is independency, i.e. when two parameters give similar contributions to the FRF, redundancy exists, which hampers the physiological interpretation. Secondly, identifiability, meaning that a parameter has to contribute to the FRF within the excited frequency band in order to assure that the influence of that parameter can be detected. The goal of model fitting is to find the most compact model with parameters that are both independent and identifiable.

During the process of model fitting, various combinations of parameter sets were estimated and validated, based on previously used models in literature (Kiemel et al., 2008; Goodworth and Peterka, 2012). In our fitting procedure, the intrinsic damping properties and the activation dynamics (mapping of EMG signals to joint torques) were found unidentifiable. Muscle-activation dynamics did not influence the magnitude of the FRF in the frequency range the data was obtained $(0.05-5 \mathrm{~Hz})$. However, the FRF phase was affected. Interaction existed with the lumped delays, which also shaped the FRF phase, which resulted in poorly interpretable values. We therefore decided not to include the muscleactivation dynamics in the model, and we described all phase changes to the lumped delays (which represents the sum of transport delay, the processing time of sensory information and an electromechanical delay). Interaction also existed in the direct terms of the FRF $\left(H_{c, \theta_{\text {ank }} 2 T_{\text {ank }}}\right.$ and $\left.H_{c, \theta_{\text {hip }} 2 T_{\text {hip }}}\right)$, between the intrinsic stiffness, the reflexive stiffness, and the lumped delay. The FRF magnitude displayed the sum of the stiffness values. The identified FRF phase was somewhere between the phase of 0 (due to the intrinsic stiffness) and the phase due to the lumped delay. The result is that only the product of reflexive stiffness and time delay was identifiable. To solve the redundancy among the three parameters, we 
assumed two of the three interacting parameters to remain constant over the five experimental conditions. The changes in intrinsic properties and lumped delays were expected to be much smaller than the changes in reflexive properties (Peterka, 2002; Cenciarini, 2010). Therefore, the intrinsic properties and lumped delays were averaged over conditions, and the reflexive stiffness was left to vary over conditions.

However, the assumption of average intrinsic stiffness over the experimental conditions might have influenced our results. Intrinsic stiffness scales with the contraction level of the muscles, which is influenced by co-contraction or other external factors (such as a force field) (Ludvig et al., 2011). With increasing force field levels, the pull of gravity on the participants' CoM was reduced, and this possibly also reduced the activation level of the muscles as control of balance became easier. With decreased muscle activation, the intrinsic stiffness will also decrease. Due to our assumption, variations in intrinsic stiffness were now captured in the reflexive stiffness values.

As mentioned before, the lumped delay in the model was estimated from the FRF phase, which resulted from the contribution of intrinsic stiffness (which acts without a delay and has a phase shift of zero) and reflexive stiffness (which acts with a delay and induced a negative phase shift). If there was an increased contribution of corrective torque due to intrinsic properties compared with reflexive feedback, the estimated lumped delay might appear to decrease (Peterka, 2002). Therefore the lumped delay parameter in the model can be better thought of as an "effective delay" rather than as a parameter representing actual delays in neural processing, transmission, and muscle activation. In case one is interested in the separate contribution of the transport delay, the electromechanical delay and the processing time in the lumped delay, electromyography measures can be of additional value.

\subsection{Conclusion}

In this study we used novel system identification techniques to derive a description of the neuromuscular control mechanisms in multi-joint balance control, by applying force disturbances at the level of the hip and between the shoulder blades. Adaptation of multijoint coordination was induced by external force fields, represented as virtual springs at the pelvis with various stiffness levels. Our results demonstrate that humans adapt to force fields by altering their postural responses, i.e. reflexive stiffness and damping. However, elderly adapted their reflexive stiffness around the ankle less compared to young participants. Furthermore, elderly had higher intrinsic ankle stiffness and larger lumped time delays of the hip. As adaptability of multi-joint coordination is an essential feature of standing balance control, in order to adjust to various conditions, this may underlie increased risk of falls with ageing. 


\title{
Chapter 6
}

\section{Reliability of system identification techniques to assess standing balance in healthy elderly}

\author{
J.H. Pasma*, D. Engelhart*, A.B. Maier, R.G.K.M. Aarts, J.M.A. van Gerven, J.H. Arendzen, \\ A.C. Schouten, C.G.M. Meskers, H. van der Kooij. *Both authors contributed equally
}

\begin{abstract}
System identification techniques have the potential to assess standing balance. By applying well-known disturbances, the contribution of the underlying systems in standing balance can be identified. In this study, we investigated the reliability of standing balance parameters obtained with multivariate closed loop system identification techniques.

Twelve healthy elderly participated in this study. Balance tests were performed twice a day during three days. First, body sway was tested using posturography during 2 minutes of standing with eyes closed. The Balance test Room (BalRoom) was used to apply four disturbances simultaneously: two sensory disturbances, to the proprioceptive and the visual system, and two mechanical disturbances applied at the leg and trunk segment. Using system identification techniques, sensitivity functions to the sensory disturbances and the neuromuscular controller were estimated. Systematic errors were assessed using linear mixed models, including trial and day and their interaction as fixed effects and participant intercept as random effect. Reliability was assessed using the generalizability theory, which allows for computing indexes of dependability (ID), standard error of the measurement (SEM) and minimal detectable change (MDC). To test validity, the BalRoom test was performed with increasing disturbance amplitude of the proprioceptive disturbance.

Results showed a systematic error between the first and second trial in the parameters describing the sensitivity functions. No systematic error was found in the neuromuscular controller and body sway. The reliability of the BalRoom parameters and body sway were moderate to excellent when the results of two trials on three days were averaged. To reach an excellent reliability of the BalRoom on one day, at least ten trials must be averaged. When the disturbance amplitude was increased, sensitivity functions to the proprioceptive disturbance decreased, whereas the sensitivity functions to the visual disturbances increased, which supports the validity of the method.

This study shows the possibility to use of system identification techniques to assess standing balance in elderly. As a systematic error was shown between the first and second trial on one day, it is concluded that assessment of steady state balance needs a training session and at least ten trials on one day to reach an excellent reliability.
\end{abstract}




\subsection{Introduction}

Impaired standing balance is a significant problem in elderly (Jonsson et al., 2004; Lin and Bhattacharyya, 2012) and is one of the main risk factors and causes of falling (Rubenstein, 2006; Muir et al., 2010). Falls often result in serious injuries, and also in death (Cummings et al., 1985). In standing balance, several underlying systems (i.e. muscles, neural system and sensory systems) interact, which results in a closed loop system in which cause and effect are interrelated (Engelhart et al., 2014). The underlying systems deteriorate with age and are influenced by diseases and medication use (Horak et al., 1989; Manchester et al., 1989; Konrad et al., 1999; Sturnieks et al., 2008). Due to redundancy, these systems can compensate for each other's deterioration. Therefore, the underlying cause of impaired standing balance is difficult to detect and hence, to intervene with targeted therapies (Pasma et al., 2014b).

Current clinical balance tests do not take aforementioned cause and effect relations and redundancy of standing balance into account and therefore cannot detect the underlying cause of impaired standing balance (Pasma et al., 2014b). Previous research showed that system identification techniques are useful to assess the underlying systems of standing balance, in which the response to well-known disturbances are assessed (Engelhart et al., date unknown; Peterka, 2002; Cenciarini, 2010; Pasma et al., 2012; Boonstra et al., 2013). A clear advantage is that this method takes into account the cause and effect relation and separates the contribution of the underlying systems. This gives the opportunity to improve diagnosis of impaired balance and, eventually, to prevent falling by targeted interventions (Engelhart et al., 2014). Before introducing the method into clinical practice for diagnosing or monitoring treatment of impaired balance, it is important to assess the reliability of this technique and compare it with posturography, which is yet unknown.

In this study we investigated the reliability of standing balance parameters obtained with four disturbances applied simultaneously and system identification techniques to assess standing balance in healthy elderly and compared this with a parameter obtained with posturography, namely body sway. We used the generalizability theory (G theory) (Shavelson and Webb, 1991), which takes into account both systematic and random measurement errors. Furthermore, recommendations will be given for study designs to reduce the measurement errors and therefore improve the reliability.

\subsection{Materials and methods}

\section{Participants}

Twelve healthy elderly aged 70 years or older participated in this study. Participants were recruited from the database of the Center of Human Drug Research, Leiden, the Netherlands, and the MyoAge study database of the Leiden University Medical Center, Leiden, the Netherlands. Participants were screened before entry to the study. Participants were excluded in case of low cognitive function (Mini Mental State Examination (MMSE) score $\leq 26$ (Folstein et al., 1975)), presence of clinical significant morbidity (haematological, 100 
renal, endocrine, pulmonary, gastrointestinal, cardiovascular, hepatic, psychiatric, neurological, musculoskeletal or allergic disorders), presence of orthostatic hypotension and use of medication. This study was approved by the Medical Ethics Committee of the Leiden University Medical Center, Leiden, the Netherlands, and was performed according to the principles of the Declaration of Helsinki and the International Conference on Harmonization/Good Clinical Practice (ICH/GCP). All participants gave written informed consent before entry to the study.

\section{Participant characteristics}

Prior to participation, a screening procedure was performed. Medical history was recorded including general questions about smoking, alcohol use, medication use and information on diseases. Anthropometric data included height and body composition measured with a bioelectrical impedance analysis (BIA, InBody 720, Biospace Co., Ltd, Seoul, Korea). Cognitive function was assessed with the MMSE (Folstein et al., 1975). Orthostatic hypotension was assessed by measuring blood pressure after at least 5 minutes in supine position and 3 minutes after postural change to standing position. Handgrip strength was measured using the Jamar dynamometer handle (Jamar, Sammons Preston Inc, Bolingbrook, IL, USA). Physical functioning was measured with the Short Physical Performance Battery (SPPB) (Guralnik et al., 1994). Walking speed was determined by a 4 meter walking test at normal pace, as part of the SPPB.

\section{Apparatus}

Standing balance was assessed using the Balance test Room (BalRoom), a custom-made device applying specifically designed disturbances during stance (Motekforce Link, Culemborg, the Netherlands, and University of Twente, Enschede, the Netherlands) (Figure 6.1). The BalRoom consists of three separated modules. The first module consists of two support surfaces (SS), which are independently actuated and rotate around the ankles (Schouten et al., 2011). By rotation of the SS around the ankle axis the proprioceptive information of the ankle is disturbed. The second module is a visual scene (VS) in front of the participant, which rotates around the ankle axes. Rotating the VS around the ankle axis results in a disturbance of the visual information. The third module consists of two rods applying forces at hip and shoulder level (FH and FS, respectively) resulting in movements around the ankle and hip joint. These disturbances are used to investigate the contribution of the ankles and hips and their coupling to standing balance (Engelhart et al., date unknown). All disturbances can be applied simultaneously. The body sway was measured in a single plane using a string potentiometer (Celesco SP2-50, Celesco, Chatsworth, CA, United States), which integrates the amplitude of unidirectional body movement transferred through a string attached to the waist of the participant. 


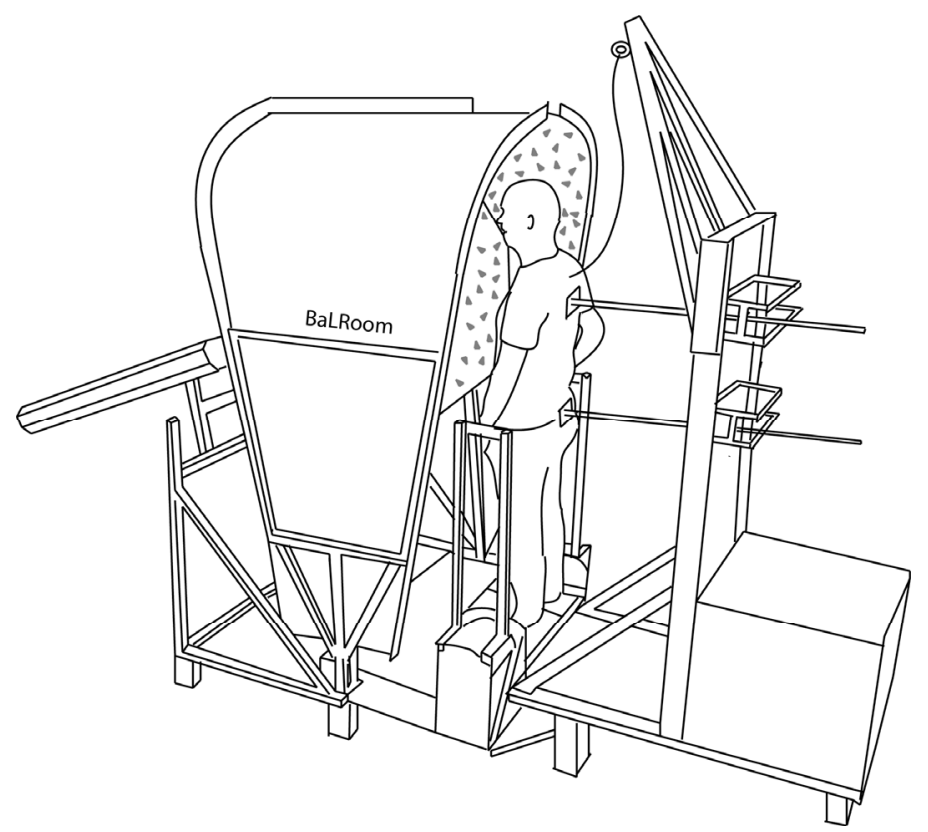

Figure 6.1: Schematic set up of the Balance test Room consisting of three modules; 1) a visual scene to apply disturbances to the visual system (VS rotation), 2) support surfaces to apply disturbances to the proprioceptive system (SS rotation), and 3) two rods to apply mechanical disturbances by giving pushes and pulls at hip and shoulder level (FH and FS).
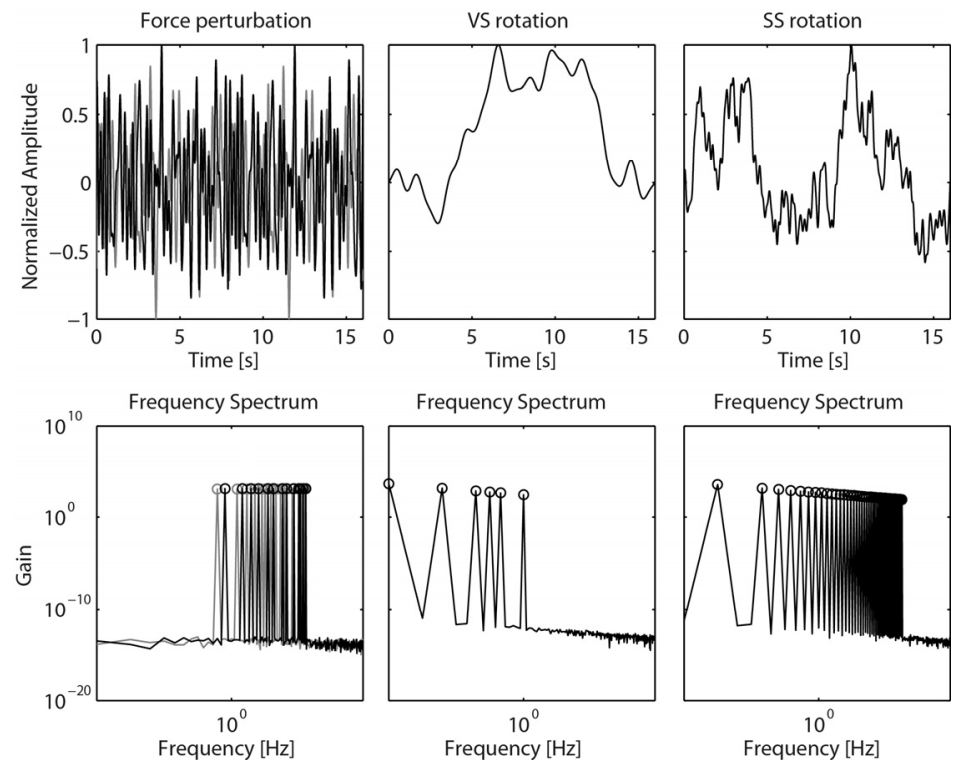

Figure 6.2: Normalized time signals and frequency spectra of the disturbances of the support surface (SS) rotation, the visual scene (VS) rotation and the rods applying forces at hip and shoulder level (FH (grey) and FS (black), respectively). 


\section{Disturbance signals}

All disturbances were multisine signals with a unique combination of frequencies (Figure 6.2). All excited frequencies were multiples of the frequency $0.0625 \mathrm{~Hz}$ resulting in a disturbance period of $16 \mathrm{~s}$. The SS rotated following a continuous position disturbance signal with increasing zero-to-peak amplitude over trials, i.e. $0.02,0.03$ and 0.04 radians, and a flat velocity spectrum with frequencies between 0.125 and $6.9375 \mathrm{~Hz}$. The VS rotated following a continuous position disturbance signal with constant zero-to-peak amplitude of 0.03 radians over trials and a flat velocity spectrum with frequencies between 0.0625 and 1 $\mathrm{Hz}$. The FH and FS disturbances are independent continuous force disturbance signals with constant zero-to-peak amplitude of 30 Newton over trials consisting of frequency contents between 0.75 and $7 \mathrm{~Hz}$. All disturbances were repeated eight times resulting in a total duration of 128 seconds.

\section{Procedure}

During the screening visit for inclusion up to 21 days before the start of the study, each participant had a training session to familiarize with the BalRoom test and disturbances and with the body sway test. No data were recorded. During the study, the tests were performed during three sessions separated by one week, allowing assessment of intersession variability. Per session the tests were performed twice separated by one hour, allowing assessment of intrasession variability. During all tests the participant wore comfortable flat shoes. During the BalRoom test, the participant was instructed to stand with the arms resting along the body, with both feet in place on the support surfaces. The two sensory (SS and VS) and mechanical (FH and FS) disturbances were applied simultaneously. Each test consisted of three conditions with increasing disturbance amplitude of the SS rotation (i.e. $0.02,0.03$ and 0.04 radians), while the amplitudes of the VS, FH and FS disturbances remained constant. The three conditions were presented in random order. Before recording each condition the participant was allowed about 10 seconds to get accustomed to the disturbances. Between conditions, the participant was offered ample resting time depending on individual needs. The participant wore a safety harness to prevent falling, which did not constrained movement nor provided support or orientation information. During the body sway test, the participant was asked to stand still and comfortable for a period of 2 minutes, with the feet approximately $10 \mathrm{~cm}$ apart and the hands in a relaxed position along the body and eyes closed.

\section{Data recording and processing}

The actual angles of SS rotation (i.e. motor angles), applied forces at hip and shoulder level (FH and FS) and the applied torques to the SS (i.e. motor torques) were available for measurement. Lower and upper body segmental movements were measured in anteriorposterior direction using two draw wire potentiometers (Celesco SP2-50, Celesco, Chatsworth, CA, United States) at a sample frequency of $1000 \mathrm{~Hz}$. The potentiometers were connected to the hip and the shoulders by magnets and straps. The motor angles, segment angles, motor torques and applied FH and FS forces, were recorded using a Matlab interface with a sample frequency of $1000 \mathrm{~Hz}$. Data analysis was performed with Matlab (The 
MathWorks, Natick, MA, United States). The leg and hip angle were calculated using goniometry and using the segment movement of the lower and upper body (Winter et al., 1990). The ankle torque was obtained by subtracting the contribution of the mass and inertia of the support surfaces from the recorded motor torques. The hip torque was obtained using the applied FH and FS forces and leg and hip angles using inverse dynamics (Winter et al., 1990). The time series were segmented into eight data blocks of 16 seconds (i.e. the period of the disturbance signal).

\section{Data analysis}

To indicate the effect of the disturbances on the ankle torque, hip torque and joint angles, Frequency Response Functions (FRFs) were estimated. The time series of the disturbances, ankle torque, hip torque, leg and hip angle were transformed to the frequency domain. The periodic part of the frequency coefficients was determined by averaging over the data blocks. The Power Spectral Densities (PSD) and Cross Spectral Densities (CSD) were computed to calculate the FRFs (van der Kooij et al., 2005). For each disturbance, only the excited frequencies were analyzed.

\section{Sensitivity functions}

The sensitivity functions of the ankle torque, hip torque, leg angle and hip angle to the SS rotation and the VS rotation were estimated using the indirect approach using equation 6.1 (Peterka, 2002; van der Kooij et al., 2005).

$$
{ }^{d} S_{x}(f)=\Phi_{d, x}(f)\left[\Phi_{d, d}(f)\right]^{-1}
$$

In which $\Phi_{d, x}$ represents the CSD of the disturbance ( $d$ ) (i.e. SS rotation or VS rotation) and $x$, which represents the ankle torque $\left(T_{a}\right)$, hip torque $\left(T_{h}\right)$, leg angle $\left(\theta_{l}\right)$, or hip angle $\left(\theta_{h}\right)$, and $\Phi_{d, d}$ the PSD of the disturbance. This results in 8 FRFs; 1$)$ SS rotation to ankle torque $\left.\left({ }^{\mathrm{SS}} S_{T_{a}}\right), 2\right) \mathrm{SS}$ rotation to hip torque $\left.\left({ }^{\mathrm{SS}} S_{T_{h}}\right), 3\right) \mathrm{SS}$ rotation to leg angle $\left({ }^{\mathrm{SS}} S_{\theta l}\right)$, and 4$) \mathrm{SS}$ rotation to hip angle $\left({ }^{S S} S_{\theta h}\right)$, and 5$)$ to 8 ) the VS rotation to each torque and angle $\left({ }^{\mathrm{VS}} S_{T_{a}},{ }^{\mathrm{VS}} S_{T_{h}},{ }^{\mathrm{VS}} S_{\theta l},{ }^{\mathrm{VS}} S_{\theta h}\right)$. Each FRF is represented by a magnitude and phase representing the ratio between the input and output and the relative timing both as function of frequency. The magnitude of the sensitivity function of the ankle and hip torque is normalized to the gravitational stiffness $\left(\mathrm{mgl}_{\mathrm{CoM}}\right)$. The average magnitude on the low frequencies ( $<0.375 \mathrm{~Hz}$ and $<0.1875 \mathrm{~Hz}$, for SS and VS respectively) and the phase on higher frequencies $(0.68 \mathrm{~Hz}$ and $0.375 \mathrm{~Hz}$, for SS and VS respectively) are the parameters of interest. Different values of frequencies were used for SS and VS due to differences in frequency content. They represent the sensitivity to the disturbances and the time lag between the disturbance and the reaction of the body, respectively, resulting in 16 parameters. 


\section{Neuromuscular controllers}

The neuromuscular controller is the link between the sensory systems and the muscles, where the sensory information is combined and muscle commands are generated to keep the body in upright position. The multi-input-multi-output (MIMO) approach was used to estimate the ankle and hip controller according to the method described by Engelhart et al. (2014) and equation 6.2 (Engelhart et al., date unknown).

$$
H_{c}(f)=-\Phi_{d, T}(f)\left[\Phi_{d, \theta}(f)\right]^{-1}
$$

In which $\Phi_{d, T}$ and $\Phi_{d, \theta}$ are the CSD matrices between the external disturbance $(d)$ (i.e. FH and FS)) and the corrective ankle and hip torques $(T)$ and the leg and hip angles $(\theta)$ resulting in a two-by-two FRF matrix $\left(H_{c}\right)$. This results in $\left.4 \mathrm{FRFs} ; 1\right)$ ankle torque to ankle angle $\left.\left(H_{T a 2 \theta l}\right), 2\right)$ ankle torque to hip angle $\left.\left(H_{T a 2 \theta h}\right), 3\right)$ hip torque to hip angle $\left(H_{T h 2 \theta h}\right)$, and 4) hip torque to ankle angle $\left(H_{T h 2 \theta l}\right)$. The magnitude is normalized to the gravitational stiffness $\left(m g l_{C o M}\right)$. The average magnitude on the low frequencies $(<1 \mathrm{~Hz})$ and the phase on higher frequencies $(2.3 \mathrm{~Hz})$ are the parameters of interest and represent the normalized effective stiffness and the time lag between the torques and angles, resulting in 8 parameters (Engelhart et al., date unknown).

\section{Body sway}

The body sway (xBS) was measured over 2 minutes. The movement of the body was expressed as millimeters of sway.

\section{Statistical analysis}

The characteristics of the participants were represented by mean and standard deviation in case of a Gaussian distribution. Else, median and inter quartile range or number and percentage were presented. The parameters obtained with system identification techniques (i.e. sensitivity and time lag of the sensitivity functions, and normalized effective stiffness and time lag of the neuromuscular controller) and body sway are given as mean and standard deviation.

Reliability was assessed using the $\mathrm{G}$ theory in three steps (Shavelson and Webb, 1991). First, systematic errors were identified using linear mixed models with trial (intrasession), day (intersession) and their interaction as fixed effects and participant intercept as random effect. The various sources of measurement errors were assessed using a random effects repeated measures analysis of variance (ANOVA) including participant, trial, day and their interactions. This resulted in the variance of the participants $\left(\sigma_{p}^{2}\right)$, the variance of the trials $\left(\sigma_{t}^{2}\right)$, the variance of the day $\left(\sigma_{d}^{2}\right)$, the variance of their interactions $\left(\sigma_{p t}{ }^{2}, \sigma_{p d}{ }^{2}\right.$ and $\left.\sigma_{t d}{ }^{2}\right)$ and the variance of the residual $\left(\sigma_{p t d, e}{ }^{2}\right)$. All were presented as percentages of the total variance. Negative variance components were set to zero. The sources of variance were used to calculate the index of dependability (ID), the standard error of the measurement (SEM) and the minimal detectable change (MDC) using equation 6.3 (Shavelson and Webb, 1991; de Vet et al., 2006). In which, $n_{t}$ is the number of trials and $n_{d}$ the number of days. 


$$
\begin{gathered}
\sigma_{\Delta}^{2}=\frac{\sigma_{t}^{2}}{n_{t}}+\frac{\sigma_{d}^{2}}{n_{d}}+\frac{\sigma_{p t}^{2}}{n_{t}}+\frac{\sigma_{p d}^{2}}{n_{d}}+\frac{\sigma_{t d}^{2}}{n_{t} n_{d}}+\frac{\sigma_{p t d, \varepsilon}^{2}}{n_{t} n_{d}} \\
I D=\frac{\sigma_{\mathrm{p}}^{2}}{\sigma_{\mathrm{p}}^{2}+\sigma_{\Delta}^{2}} \\
S E M=\sqrt{\sigma_{\Delta}^{2}} \\
M D C=1.96 * \sqrt{2} * S E M
\end{gathered}
$$

Comparable with an intraclass correlation coefficient (ICC), the ID ranges between 0 and 1 and can be interpreted as; ID $<0.40$ represents poor reliability, $0.40<$ ID $<0.75$ represents moderate reliability, and ID > 0.75 represents excellent reliability (Fleiss, 1986). The SEM indicates the absolute reliability and is represented by an absolute value and a percentage of the overall mean. The MDC shows which effect (e.g. treatment effect) can be detected with the parameters of interest and therefore indicates the clinical relevance. A low SEM and MDC are indicative of a reliable and clinical relevant parameter.

Second, a decision study was performed in which the effect of different measurement protocols on the reliability was investigated. Aforementioned equations show that increasing the number of trials or number of days results in an increase of ID and a decrease of SEM and MDC, i.e. an improvement of reliability. In the decision study, the number of trials was varied between 1 and 40 trials and the number of days between 1 and 3. Per number of days, the number of trials needed to reach an excellent reliability was determined in this group of healthy elderly (ID > 0.75).

Third, a validity study was performed to assess whether differences in the sensitivity functions represented by the sensitivity and time lag due to increasing disturbance amplitude of the SS rotation could be detected. A linear mixed model was constructed with disturbance amplitude as fixed effect and participant intercept as random effect.

Statistical analysis was performed with SPSS version 20 (SPSS Inc., Chicago, USA). Graphs were made with Matlab (The MathWorks, Natick, MA, United States). 


\subsection{Results}

\section{Participant characteristics}

Table 6.1 presents the characteristics of the healthy old participants. Figure 6.3, Figure 6.4 and Figure 6.5 displays the FRFs of the sensitivity functions to the SS rotations and the VS rotations, and of the neuromuscular controller.

\section{Systematic errors}

Table 6.2 reports the systematic errors. No systematic errors were found for the body sway $\left(x_{B S}\right)$. The sensitivity functions show both a main effect of trial and day. Overall, the sensitivity to the SS rotation was lower during the first trial compared with the second trial in the sensitivity functions (i.e. ${ }^{S S} S_{\theta h_{1}}{ }^{S S} S_{\theta l}$, and ${ }^{S S} S_{T a}$ ) and it was lower during the first day compared to the second and third day (for ${ }^{{ }^{S}} S_{T h}$ and ${ }^{S S} S_{T_{a}}$ ). The sensitivity function to the VS rotation shows the opposite result; the sensitivity of the first trial was higher compared with the second trial for all sensitivity functions $\left({ }^{{ }^{S} S} S_{\theta h l}{ }^{{ }^{2} S_{\theta l l}}{ }^{{ }^{S} S} S_{T h}\right.$ and $\left.{ }^{{ }^{2} S} S_{T a}\right)$. Furthermore, the time lags of some sensitivity functions (i.e. ${ }^{S S} S_{T a r}$ and ${ }^{V S} S_{\theta l}$ ) were higher in the first trial compared with the second trial. The time lags of ${ }^{v S} S_{T a}$ and ${ }^{v S} S_{T h}$ were also lower during the first day compared with the third day.

The normalized effective stiffness estimated using the FS and FH disturbances showed an effect of the trial; one component of the neuromuscular controller $\left(H_{\theta 12 T h}\right)$ was lower during the first trial compared with the second trial. No effect of trial and day was found for the time lags of all components of the neuromuscular controller.

Table 6.1: Participant characteristics. All parameters are presented as mean with standard deviation unless indicated otherwise. BMI: body mass index, MMSE: Mini Mental State Examination, SPPB: Short Physical Performance Battery, IQR: inter quartile range.

\begin{tabular}{lr}
\hline & All $(\mathbf{n}=\mathbf{1 2})$ \\
\hline Age, years & $73.3(3.4)$ \\
Men (n, \%) & $6(50)$ \\
Anthropometry & \\
Weight, kg & $72.2(9.1)$ \\
Height, $\mathrm{m}$ & $1.70(0.08)$ \\
BMI, kg/m² & $24.9(2.4)$ \\
Health characteristics & \\
Number of medication, median (IQR) & $0(0-0)$ \\
MMSE, points; median (IQR) & $29(28-30)$ \\
Physical functioning & \\
Handgrip strength, kg & $34.7(8.6)$ \\
Gait speed, m/s & $1.28(0.16)$ \\
SPPB, points; median (IQR) & $11(10-12)$
\end{tabular}




\section{Variance components}

Table 6.3 shows the magnitude of the variance components as percentage of the total variance. Variance of the participant $\left(\sigma_{p 2}\right)$ was on average $20.8 \%$ and varied between $0.0 \%$ and $87.3 \%$. The contribution of the trial variance $\left(\sigma_{t 2}\right)$ was on average $6.6 \%$ and varied between $0.0 \%$ and $28.8 \%$. The contribution of the day variance $\left(\sigma_{d 2}\right)$ was on average $2.5 \%$ and varied between $0.0 \%$ and $12.5 \%$.

The error variance related to the interactions between the participant and trial $\left(\sigma_{p t 2}\right)$, between participant and day $\left(\sigma_{p d 2}\right)$ and between trial and day $\left(\sigma_{t d 2}\right)$ were low; on average they were $4.3 \%, 13.4 \%$ and $4.4 \%$, respectively.

The largest proportion of measurement variability was due to the participant variability $\left(\sigma_{p}^{2}\right)$ and the other interactions combined with the residual error $\left(\sigma_{p t d, e}{ }^{2}\right)$ contributing on average $48.0 \%$ ranging from $7.2 \%$ to $80.8 \%$.

\section{Reliability}

Table 6.4 presents the results of the reliability measures. In this study design, the ID of 4 out of 25 parameters was higher than 0.75 and in 12 out of 25 parameters ID was between 0.40 and 0.75 . The SEM and SEM \% were inverse related with the ID. Furthermore, the MDC was lower with increased ID. To reach an ID of 0.75 , for $32 \%(8 / 25)$ of the parameters at least ten trials are needed to average over one day. Increasing the number of days resulted in less trials needed per day to reach an ID higher than 0.75 .

\section{Validity}

Table 6.5 presents the results of the validity study. The mean and standard deviation of the parameters of the second trial at the first day are given for each condition. All sensitivities to the SS rotation decreased with increasing disturbance amplitude and all sensitivities to the VS rotation increased with increasing disturbance amplitude. No significant differences were found for the time lag of the sensitivities to the SS rotation and VS rotation. No significant differences were found between the conditions for the parameters describing the neuromuscular controller. 
Day 1
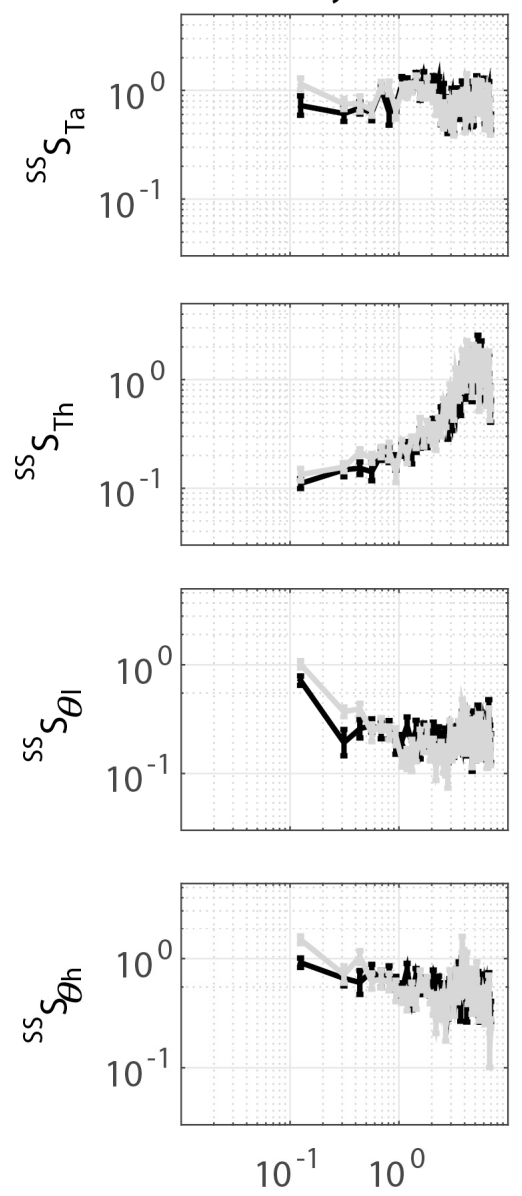

Frequency $[\mathrm{Hz}]$
Day 2
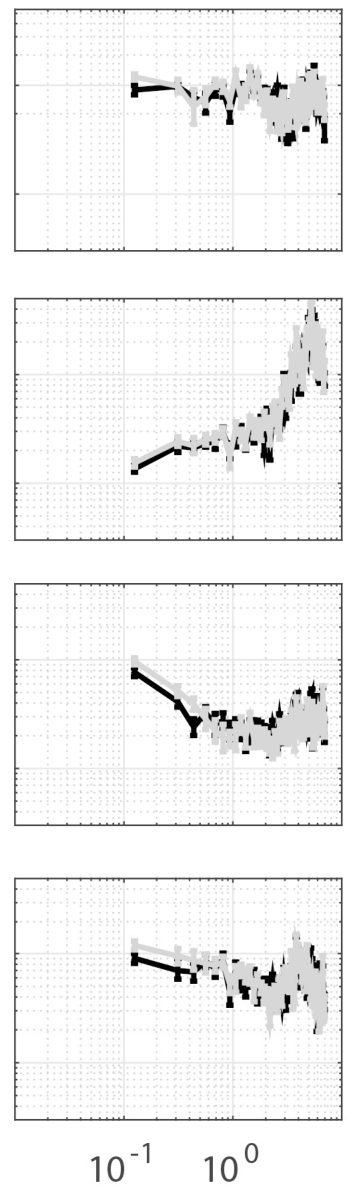

Frequency $[\mathrm{Hz}]$
Day 3
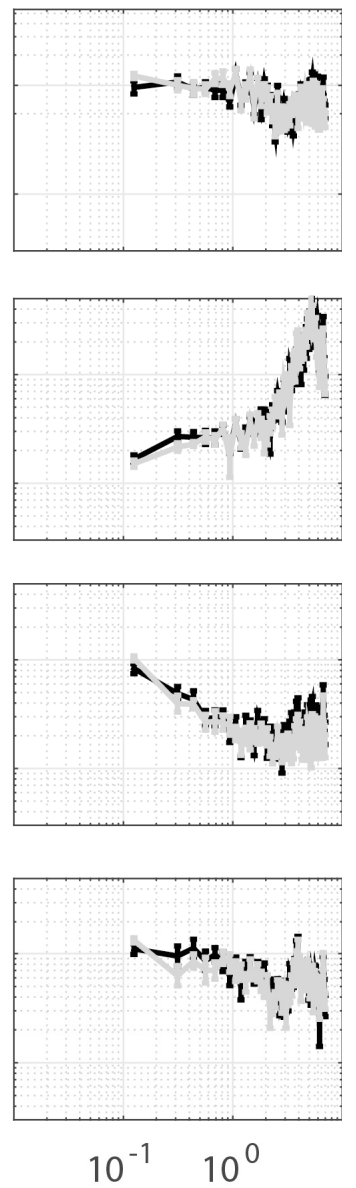

Frequency $[\mathrm{Hz}]$

Figure 6.3: Average sensitivity functions of the ankle torque $\left({ }^{S S} S_{T a}\right)$, hip torque $\left({ }^{S S} S_{T h}\right)$, leg angle $\left({ }^{S S} S_{\theta l}\right)$ and hip angle $\left({ }^{S S} S_{\theta h}\right)$ to the rotation of the support surfaces per day per trial (trial 1 in black and trial 2 in grey) are presented by mean and standard error, only magnitude is shown. 
Day 1
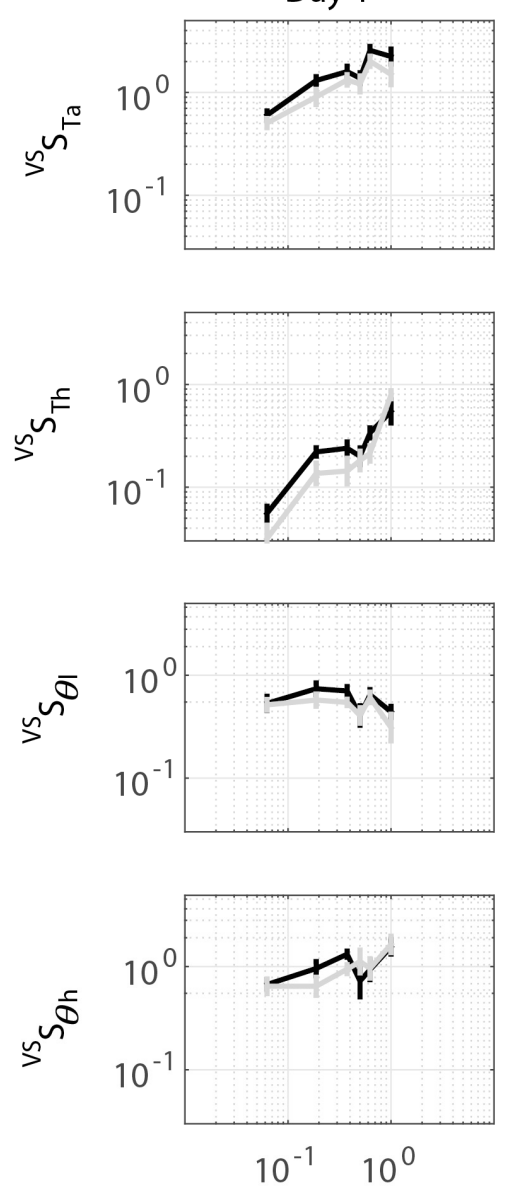

Frequency $[\mathrm{Hz}]$
Day 2
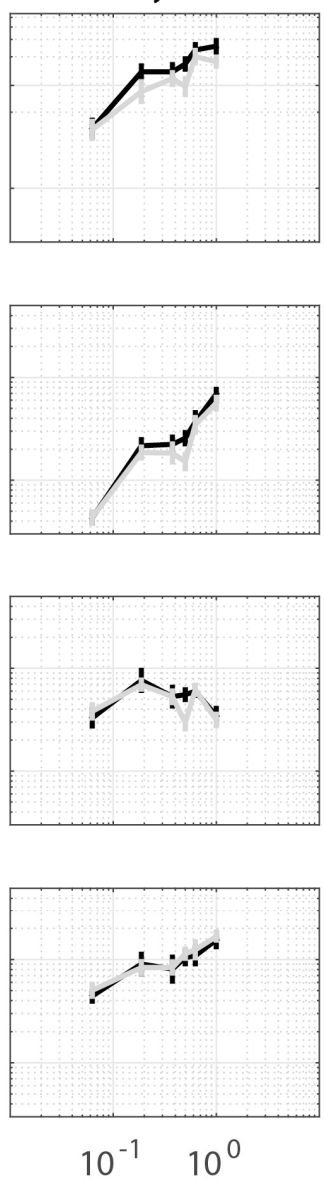

Frequency [Hz]
Day 3
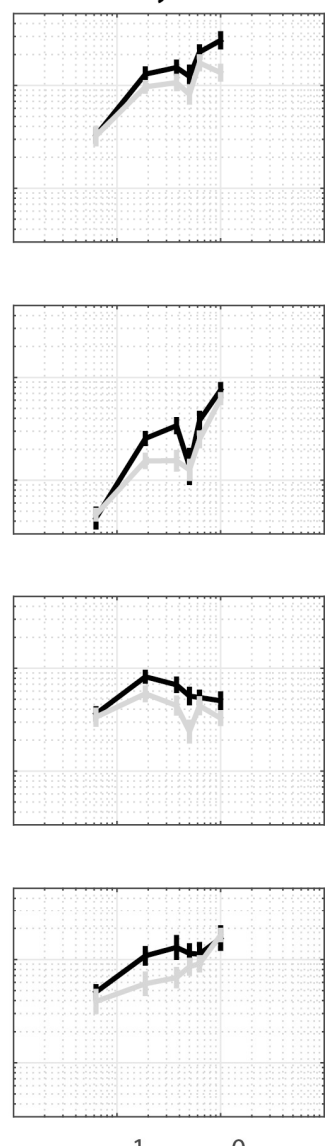

$10^{-1} \quad 10^{0}$

Frequency $[\mathrm{Hz}]$

Figure 6.4: Average sensitivity functions of the ankle torque $\left({ }^{V S} S_{T a}\right)$, hip torque $\left({ }^{V S} S_{T h}\right)$, leg angle $\left({ }^{V S} S_{\theta l}\right)$ and hip angle $\left({ }^{V S} S_{\theta h}\right)$ to the rotation of the visual scene per day per trial (trial 1 in black and trial 2 in grey) are presented by mean and standard error, only magnitude is shown. 
Day 1
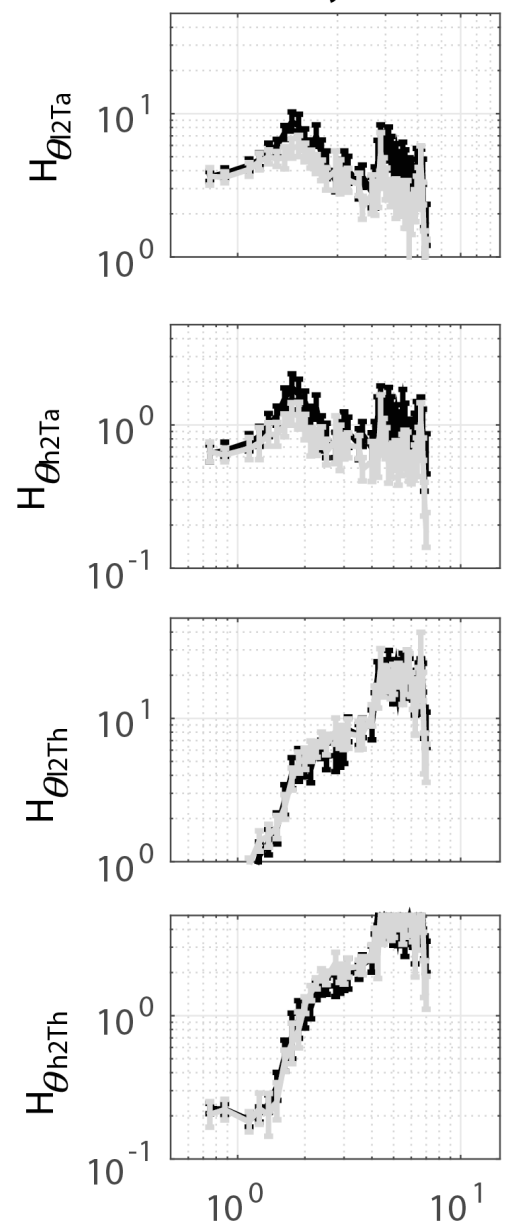

Frequency $[\mathrm{Hz}]$
Day 2
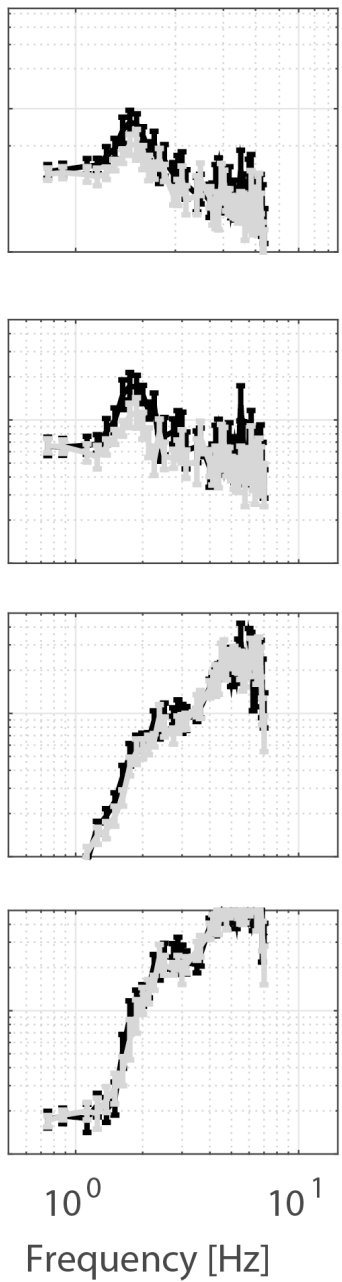

Day 3
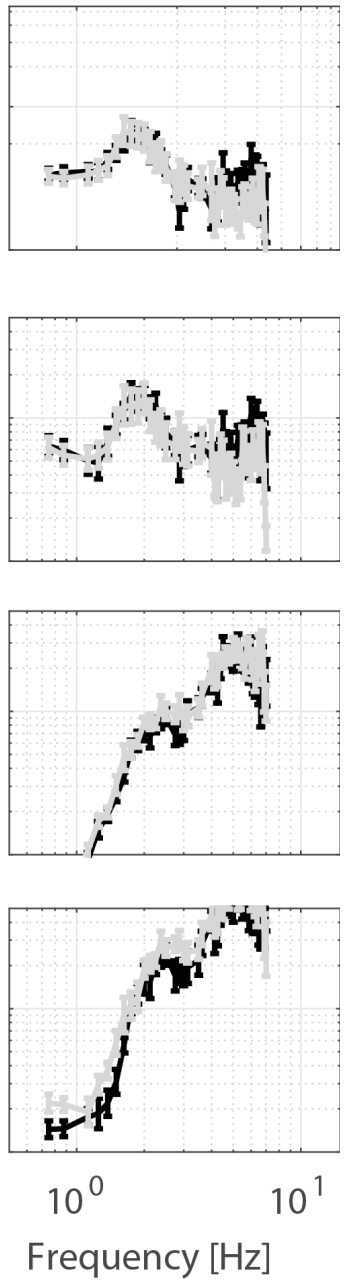

Figure 6.5: Average Frequency Response Functions of the neuromuscular controller (i.e. $H_{\text {el2Tar }} H_{\text {en2Tar }}$ $H_{\theta / 2 T h}, H_{\theta h 2 T h}$ ) per day per trial (trial 1 in black and trial 2 in grey) are presented by mean and standard error, only magnitude is shown. 
Table 6.2: Systematic errors of all parameters using linear mixed model with day, trial and their interaction as fixed effect and subject intercept as random effect. Significant differences identified in bold. n.s. : not significant.

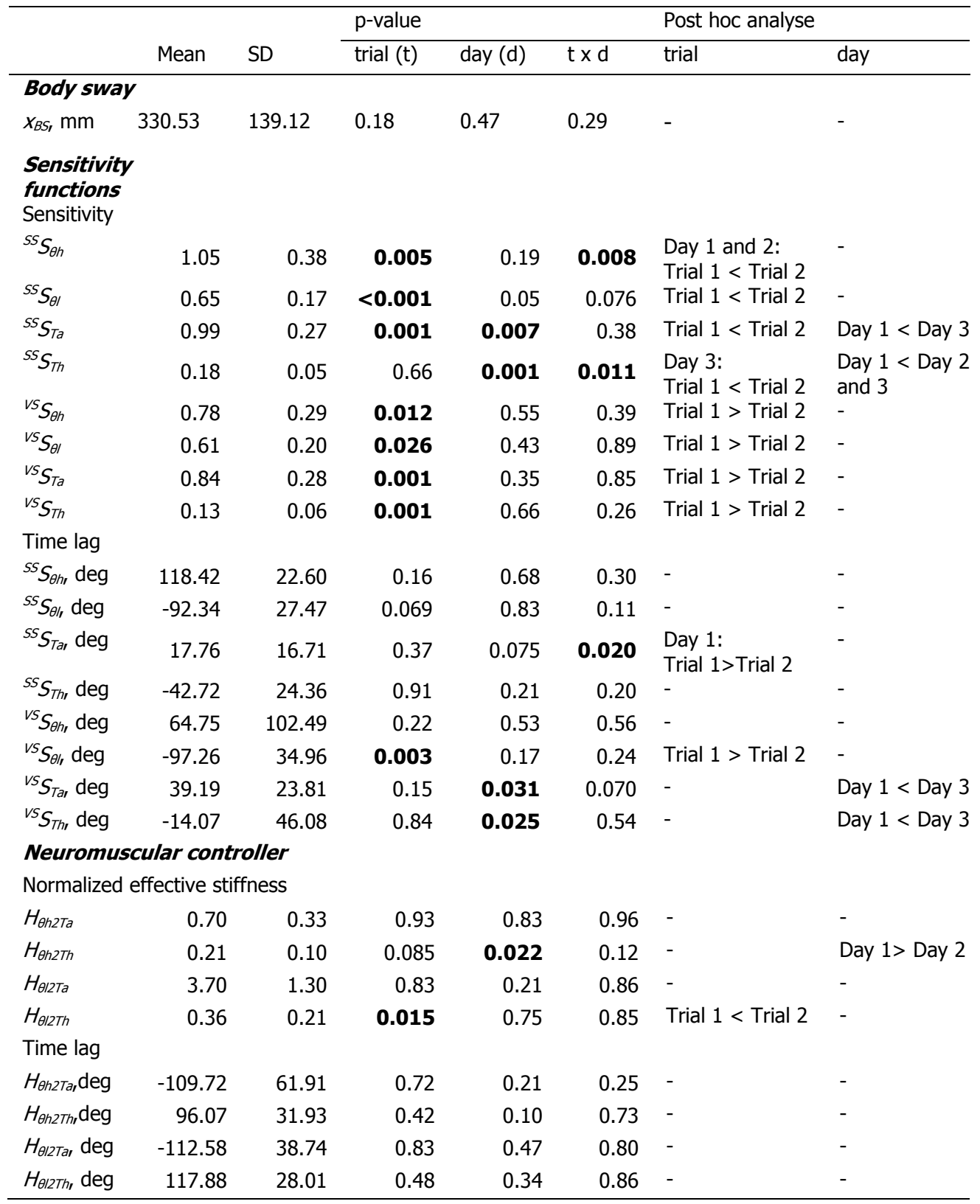


Table 6.3: Relative magnitude of the variance components obtained with the G-study for all parameters obtained with system identification techniques.

\begin{tabular}{|c|c|c|c|c|c|c|c|}
\hline & $\begin{array}{l}\text { participant } \\
\text { (p), \% }\end{array}$ & $\begin{array}{l}\text { trial } \\
(\mathrm{t}), \%\end{array}$ & $\begin{array}{l}\text { day } \\
\text { (d), \% }\end{array}$ & $\begin{array}{l}p \times t, \\
\%\end{array}$ & $\begin{array}{l}\mathrm{p} \times \mathrm{d}, \\
\%\end{array}$ & $\begin{array}{l}\mathrm{t} \times \mathrm{d}, \\
\%\end{array}$ & $\begin{array}{l}p \times t \times d, e, \\
\%\end{array}$ \\
\hline \multicolumn{8}{|c|}{ Body sway } \\
\hline$x_{B S,} \mathrm{~mm}$ & 87.3 & 0.2 & 0.0 & 0.0 & 4.7 & 0.5 & 7.2 \\
\hline \multicolumn{8}{|c|}{ Sensitivity functions } \\
\hline \multicolumn{8}{|c|}{ Sensitivity } \\
\hline${ }^{s S} S_{\theta h}$ & 24.9 & 5.5 & 0.0 & 3.1 & 8.7 & 20.7 & 37.1 \\
\hline${ }^{s S} S_{\theta 1}$ & 11.0 & 28.8 & 0.0 & 4.7 & 9.4 & 9.8 & 36.5 \\
\hline${ }^{S S} S_{T a}$ & 19.6 & 15.5 & 6.7 & 0.0 & 11.0 & 2.2 & 44.9 \\
\hline${ }^{s S} S_{T h}$ & 11.2 & 0.0 & 12.5 & 4.0 & 23.2 & 16.9 & 32.1 \\
\hline${ }^{v s} S_{\theta h}$ & 26.0 & 10.1 & 0.3 & 0.0 & 0.0 & 0.0 & 63.6 \\
\hline${ }^{v s} S_{\theta 1}$ & 0.0 & 16.2 & 0.0 & 22.2 & 6.7 & 0.0 & 54.9 \\
\hline${ }^{v S} S_{T a}$ & 0.0 & 23.7 & 3.5 & 19.3 & 17.2 & 0.0 & 36.2 \\
\hline${ }^{{ }^{V}} S_{T h}$ & 39.1 & 16.7 & 0.0 & 0.0 & 5.9 & 3.6 & 34.8 \\
\hline \multicolumn{8}{|l|}{ Time lag } \\
\hline${ }^{S S} S_{\theta h}$ & 27.7 & 1.6 & 0.0 & 1.4 & 0.0 & 6.3 & 63.0 \\
\hline${ }^{S S} S_{\theta l}$ & 6.9 & 6.4 & 0.0 & 0.0 & 0.0 & 5.9 & 80.8 \\
\hline${ }^{S S} S_{T a}$ & 18.9 & 0.0 & 0.0 & 6.0 & 12.8 & 17.2 & 45.1 \\
\hline${ }^{s s} S_{T h}$ & 29.6 & 0.0 & 0.0 & 0.0 & 9.4 & 6.0 & 55.0 \\
\hline${ }^{v s} S_{\theta h}$ & 7.5 & 5.4 & 6.0 & 13.5 & 14.3 & 0.0 & 53.3 \\
\hline${ }^{v S} S_{\theta l}$ & 3.9 & 16.0 & 0.0 & 12.7 & 21.3 & 4.2 & 41.9 \\
\hline${ }^{v S_{S}} S_{T a}$ & 41.8 & 0.0 & 2.3 & 0.0 & 0.0 & 5.1 & 50.9 \\
\hline${ }^{v S} S_{T h}$ & 20.3 & 0.0 & 10.0 & 0.2 & 11.9 & 0.8 & 56.8 \\
\hline \multicolumn{8}{|c|}{ Neuromuscular controller } \\
\hline \multicolumn{8}{|c|}{ Normalized effective stiffness } \\
\hline$H_{\text {en2Ta }}$ & 48.1 & 1.2 & 1.8 & 0.0 & 0.0 & 0.0 & 48.9 \\
\hline$H_{\theta h 2 T h}$ & 34.6 & 1.7 & 2.5 & 0.0 & 4.7 & 5.0 & 51.5 \\
\hline$H_{\text {el/2Ta }}$ & 48.9 & 1.0 & 3.4 & 0.0 & 0.0 & 0.0 & 46.8 \\
\hline$H_{\text {Ol/2Th }}$ & 21.7 & 9.0 & 2.4 & 0.0 & 0.0 & 0.0 & 66.9 \\
\hline \multicolumn{8}{|l|}{ Time lag } \\
\hline$H_{\theta h 2 T a}$ & 18.4 & 0.0 & 0.0 & 0.0 & 33.5 & 0.3 & 47.8 \\
\hline$H_{\theta h 2 T h}$ & 7.8 & 0.0 & 6.0 & 7.9 & 55.3 & 1.2 & 21.7 \\
\hline$H_{\text {OIITa }}$ & 19.0 & 0.0 & 0.0 & 4.9 & 25.2 & 0.0 & 50.9 \\
\hline$H_{\theta \mid 2 T h}$ & 13.4 & 0.0 & 1.4 & 4.2 & 50.1 & 0.5 & 30.3 \\
\hline Mean & 20.8 & 6.6 & 2.5 & 4.3 & 13.4 & 4.4 & 48.0 \\
\hline
\end{tabular}


Table 6.4: Reliability statistics of the sensitivity functions and neuromuscular controller. Abbreviations: ID, Index of Dependability ( $>0.75$ identified in bold); SEM, Standard Error of Measurement; MDC, Minimal Detectable Change.

\begin{tabular}{|c|c|c|c|c|c|c|c|}
\hline & ID & SEM & SEM \% & MDC & $\begin{array}{c}\text { trials / } 1 \\
\text { day } \\
>0.75 \\
\end{array}$ & $\begin{array}{c}\text { \# trials / } 2 \text { days } \\
>0.75\end{array}$ & $\begin{array}{r}\# \text { trials / } 3 \\
>0.75\end{array}$ \\
\hline \multicolumn{8}{|c|}{ Body sway } \\
\hline$x_{B S_{\imath}} \mathrm{mm}$ & 0.97 & 25.01 & 7.57 & 69.32 & 1 & 1 & 1 \\
\hline \multicolumn{8}{|c|}{ Sensitivity functions } \\
\hline \multicolumn{8}{|c|}{ Sensitivity } \\
\hline${ }^{s S} S_{\theta h}$ & 0.60 & 0.17 & 16.23 & 0.47 & $>40$ & 6 & 3 \\
\hline${ }^{S S} S_{\theta l}$ & 0.28 & 0.10 & 15.25 & 0.27 & $>40$ & $>40$ & 32 \\
\hline${ }^{S S} S_{T a}$ & 0.48 & 0.13 & 13.45 & 0.37 & $>40$ & 22 & 6 \\
\hline${ }^{s S} S_{T h}$ & 0.34 & 0.03 & 14.86 & 0.07 & $>40$ & $>40$ & $>40$ \\
\hline${ }^{v s} S_{\theta n}$ & 0.62 & 0.13 & 17.14 & 0.37 & 8 & 4 & 3 \\
\hline${ }^{v S} S_{\theta l}$ & 0.00 & 0.12 & 19.97 & 0.34 & $>40$ & $>40$ & $>40$ \\
\hline${ }^{v S} S_{T a}$ & 0.00 & 0.19 & 22.41 & 0.52 & $>40$ & $>40$ & $>40$ \\
\hline${ }^{v s} S_{T h}$ & 0.70 & 0.03 & 19.85 & 0.07 & 5 & 2 & 2 \\
\hline \multicolumn{8}{|l|}{ Time lag } \\
\hline${ }^{s S} S_{\theta h,}$ deg & 0.68 & 8.94 & 7.55 & 24.78 & 7 & 4 & 3 \\
\hline${ }^{S S} S_{\theta l,}$ deg & 0.28 & 14.78 & 16.01 & 40.97 & 36 & 18 & 12 \\
\hline${ }^{S S} S_{T a r}$ deg & 0.52 & 7.42 & 41.79 & 20.57 & $>40$ & $>40$ & 11 \\
\hline${ }^{S S} S_{T h,}$ deg & 0.69 & 9.58 & 22.42 & 26.55 & $>40$ & 6 & 3 \\
\hline${ }^{v S} S_{\theta h}$, deg & 0.23 & 59.54 & 91.95 & 165.03 & $>40$ & $>40$ & $>40$ \\
\hline${ }^{v S} S_{\theta \prime \prime}$ deg & 0.12 & 19.85 & 20.41 & 55.03 & $>40$ & $>40$ & $>40$ \\
\hline${ }^{v S} S_{T a r}$ deg & 0.81 & 8.39 & 21.41 & 23.26 & 4 & 2 & 2 \\
\hline${ }^{v S} S_{T h}$ deg & 0.54 & 19.82 & 140.83 & 54.92 & $>40$ & 37 & 7 \\
\hline
\end{tabular}

Neuromuscular controller

Normalized effective stiffness

\begin{tabular}{|c|c|c|c|c|c|c|c|}
\hline$H_{\text {eh2Ta }}$ & 0.84 & 0.13 & 18.01 & 0.35 & 4 & 2 & 2 \\
\hline$H_{\text {eh2Th }}$ & 0.73 & 0.04 & 18.07 & 0.11 & 8 & 3 & 2 \\
\hline$H_{\theta \mid 2 T a}$ & 0.84 & 0.48 & 12.89 & 1.32 & 3 & 2 & 1 \\
\hline$H_{\theta \mid 2 T h}$ & 0.57 & 0.12 & 32.21 & 0.32 & 10 & 5 & 4 \\
\hline \multicolumn{8}{|l|}{ Time lag } \\
\hline$H_{\text {өh2Ta }}$ deg & 0.49 & 29.55 & 26.93 & 81.90 & $>40$ & $>40$ & $>40$ \\
\hline$H_{\theta h 2 T h}$ deg & 0.22 & 16.64 & 17.33 & 46.14 & $>40$ & $>40$ & $>40$ \\
\hline$H_{\theta \mid 2 T a l}$ deg & 0.50 & 17.55 & 15.59 & 48.64 & $>40$ & $>40$ & $>40$ \\
\hline$H_{\theta / 2 T h,}$ deg & 0.35 & 13.97 & 11.85 & 38.72 & $>40$ & $>40$ & $>40$ \\
\hline
\end{tabular}


Table 6.5: Mean and standard deviation of the parameters describing the sensitivity functions and the neuromuscular controller corresponding to three conditions with increasing disturbance amplitude, combined with statistical results.

\begin{tabular}{|c|c|c|c|c|c|c|c|}
\hline & \multicolumn{2}{|c|}{$0.02 \mathrm{rad}$} & \multicolumn{2}{|c|}{$0.03 \mathrm{rad}$} & \multicolumn{2}{|c|}{$0.04 \mathrm{rad}$} & \multirow[t]{2}{*}{$p$-value } \\
\hline & Mean & SD & Mean & SD & Mean & SD & \\
\hline \multicolumn{8}{|c|}{ Sensitivity functions } \\
\hline \multicolumn{8}{|l|}{ Sensitivity } \\
\hline${ }^{s s} S_{\theta h}$ & 1.13 & 0.31 & 0.94 & 0.41 & 0.82 & 0.24 & 0.003 \\
\hline${ }^{S S} S_{\theta 1}$ & 0.73 & 0.14 & 0.58 & 0.13 & 0.48 & 0.09 & $<0.001$ \\
\hline${ }^{s S} S_{T a}$ & 1.08 & 0.21 & 0.93 & 0.20 & 0.75 & 0.13 & $<0.001$ \\
\hline${ }^{s S} S_{T h}$ & 0.19 & 0.05 & 0.18 & 0.05 & 0.15 & 0.03 & 0.001 \\
\hline${ }^{v s} S_{\theta h}$ & 0.73 & 0.22 & 0.82 & 0.28 & 0.93 & 0.27 & 0.18 \\
\hline${ }^{v S} S_{\theta l}$ & 0.58 & 0.14 & 0.63 & 0.16 & 0.77 & 0.28 & 0.079 \\
\hline${ }^{v S} S_{T a}$ & 0.73 & 0.25 & 0.90 & 0.23 & 1.04 & 0.39 & 0.044 \\
\hline${ }^{{ }^{S}} S_{T h}$ & 0.12 & 0.04 & 0.14 & 0.05 & 0.17 & 0.07 & 0.015 \\
\hline \multicolumn{8}{|l|}{ Time lag } \\
\hline${ }^{s S} S_{\theta h}, \operatorname{deg}$ & 114.74 & 19.76 & 114.47 & 21.32 & 115.66 & 19.37 & 0.98 \\
\hline${ }^{S S} S_{\theta l}$ deg & -98.15 & 24.46 & -105.43 & 20.26 & -92.20 & 30.52 & 0.42 \\
\hline${ }^{S S} S_{T a r}$ deg & 16.34 & 11.98 & 15.39 & 8.90 & 22.39 & 12.54 & 0.094 \\
\hline${ }^{S S} S_{T h}$ deg & -37.66 & 32.00 & -41.71 & 12.53 & -41.50 & 15.35 & 0.81 \\
\hline${ }^{v S} S_{\theta h}$ deg & 52.80 & 117.92 & 23.45 & 140.10 & 94.19 & 42.41 & 0.32 \\
\hline${ }^{v s} S_{\theta \mid,}$ deg & -96.04 & 31.73 & -105.29 & 44.74 & -102.27 & 19.63 & 0.80 \\
\hline${ }^{{ }^{S}} S_{T a r}$ deg & 43.44 & 25.46 & 36.72 & 27.95 & 40.84 & 18.80 & 0.73 \\
\hline${ }^{v S} S_{T h r}$ deg & -0.46 & 32.38 & -9.24 & 31.00 & -9.29 & 24.15 & 0.33 \\
\hline \multicolumn{8}{|c|}{ Neuromuscular controller } \\
\hline \multicolumn{8}{|c|}{ Normalized effective stiffness } \\
\hline$H_{\text {eh2Ta }}$ & 0.70 & 0.26 & 0.80 & 0.49 & 0.75 & 0.28 & 0.61 \\
\hline$H_{\text {eh2Th }}$ & 0.18 & 0.05 & 0.20 & 0.08 & 0.19 & 0.11 & 0.74 \\
\hline$H_{\text {Ol/2Ta }}$ & 3.70 & 1.21 & 3.93 & 1.60 & 3.93 & 1.03 & 0.80 \\
\hline$H_{\theta / 2 T h}$ & 0.42 & 0.25 & 0.39 & 0.22 & 0.29 & 0.15 & 0.29 \\
\hline \multicolumn{8}{|l|}{ Time lag } \\
\hline$H_{\text {th2Tal }}$ deg & -89.99 & 73.93 & -76.50 & 66.69 & -78.23 & 78.66 & 0.89 \\
\hline$H_{\theta h 2 T h r}$ deg & 102.46 & 39.66 & 119.09 & 44.69 & 119.24 & 36.63 & 0.42 \\
\hline$H_{\theta \mid 2 T a}$ deg & -110.27 & 58.82 & -86.77 & 37.46 & -108.93 & 48.49 & 0.28 \\
\hline$H_{\theta \mid 2 T h}$ deg & 123.08 & 36.14 & 136.45 & 31.24 & 138.22 & 29.81 & 0.37 \\
\hline
\end{tabular}




\subsection{Discussion}

In this study, we assessed the reliability of a comprehensive set of parameters obtained with four disturbances applied simultaneously and (MIMO closed loop) system identification techniques describing standing balance in a group of healthy elderly. Results were obtained by measuring standing balance twice during three days. A distinction was made between systematic and random errors. The results showed a systematic error between the first and second trial with the BalRoom on one day, which was not found using body sway measurements. The reliability ranged from moderate to excellent when averaging two trials on three days. This is the first study that investigated the reliability of system identification techniques to assess standing balance in healthy elderly.

\section{Systematic errors}

In general, the sensitivity to the SS rotation was lower in the first trial compared with the second trial, while the sensitivity to the VS rotation was higher in the first trial compared to the second trial. These results are confirmed by the variance component of the trial $\left(\sigma_{t}^{2}\right)$ and day $\left(\sigma_{d}^{2}\right)$; a high variance component of trial and day indicates a systematic error. Previous studies using system identification techniques also showed a systematic error between the first and second trial or between days. These differences were explained by motor learning, changes in posture or stretching of the joints (Reeves et al., 2014; Lariviere et al., 2015; Popovich et al., 2015). In contrast, in a previous study no learning effects were found. These results might be due to the practice session all participants performed prior to participation in this study (Hendershot et al., 2012).

In this study, the differences between the first trial compared with the second trial (i.e. a lower sensitivity to proprioception and a higher sensitivity to vision during the first trial) could be explained by a difference in strategy used to maintain standing balance or familiarization during the test. According to the sensory reweighting hypothesis, sensory information is weighted based on reliability; the weight of the proprioception increased at the cost of a decrease of the weight of the other sensory information (Peterka, 2002). As the sensitivity to the disturbances represents the contribution of the proprioceptive and visual information, the sensitivity to the SS rotation increases, while the sensitivity to the VS rotation decreases. The combination of mechanical disturbances with sensory disturbances of the visual and proprioceptive information could have resulted in a longer adaptation time or a redundancy of applied strategies to withstand the disturbances. However, comparable systematic errors within a day were found in healthy elderly (unpublished data) in a previous study using only SS rotation to disturb proprioceptive information (Pasma et al. submitted), which suggest that the longer adaptation time is not due to the combination of multiple disturbances. In contrast, no systematic errors were found in healthy young adults (unpublished data). This is an indication of increased adaptation time in elderly compared with young adults. When a steady state of standing balance is to be assessed, a familiarization trial is needed on the same day to overcome the systematic error between trials. Excluding the first trial of each day resulted in less systematic errors between days. 


\section{Reliability}

The variance component of the participant $\left(\sigma_{p}^{2}\right)$ corresponds to the ICC when both $n_{t}$ and $n_{d}$ are equal to one. The reliability of the parameters ranged from poor to moderate. To increase reliability of steady state balance assessment, multiple trials on more than one day have to be performed. The ID values indicate that performing two trials on three days results in a reliability ranging from moderate to excellent needed to discriminate between healthy old individuals. A high residual variance $\left(\sigma_{p t d, e}{ }^{2}\right)$ component indicates that a majority of the measurement error is random or can be attributed to error sources not identified in the study.

In this study, relative low SEM\% were found $(<20 \%)$, which is comparable with other studies using system identification techniques (Lariviere et al., 2015). A low SEM\% indicates that the parameter could detect changes over time within the same participant (e.g. effects of intervention or changes in conditions). However, the SEM values depend on the number of trials performed on the number of days. The MDC values are in the same order as in a previous study using only SS rotation in healthy elderly (unpublished data) and indicates which change in the parameters can be minimally detected, when comparing groups or within the same participant. It is difficult to interpret the MDC results of new parameters. To get more feeling for this measure and to get more insight in the clinical relevance, it is recommended to assess standing balance using system identification techniques in several groups of elderly with a large variance in impaired balance severity and clinical phenotypes (de Vet et al., 2006).

The results showed that at least 10 trials on one day are needed to reach an excellent reliability for steady state balance assessment in almost one third of the parameters. In this study, averaging trials across days seems to be more effective than averaging more trials per day. These results are consistent with the variance component of interaction; the variance component of participant $\mathrm{x}$ day $\left(\sigma_{p d}{ }^{2}\right)$ is much higher than the variance component of participant $x$ trial $\left(\sigma_{p t}{ }^{2}\right)$. This means that the parameters for each participant were more affected by between day than within day sources of error, relative to the other participants. These results are in accordance with previous studies; Lariviere et al. (2015) also showed that one till ten trials were needed to assess an excellent reliability for parameters obtained with system identification techniques (Lariviere et al., 2015). A lower reliability seems to be a general feature of position stabilization task in contrast to tracking tasks (Popovich et al., 2015).

\section{Validity}

The validity study showed that differences could be detected within participants by changing the experimental condition. It was possible to detect changes over conditions using one trial. Increasing the disturbance amplitude of the SS rotation resulted in a decreased sensitivity to the SS rotation and an increased sensitivity to the VS rotation. This result was expected according to the sensory reweighting hypothesis, as mentioned before. Our findings are therefore also in line with previous studies investigating sensory reweighting 
during standing balance using system identification techniques (Peterka, 2002; Pasma et al., 2012). No changes were found in the neuromuscular controller by increasing the disturbance amplitude of the SS rotation. This is following our expectations, as changes in sensory information does not influence the stiffness and damping of the neuromuscular controller. These results are also in accordance with a previous study, in which we showed that the neuromuscular controller did not change with increasing disturbance amplitude of the SS rotation (Pasma et al., 2012).

\section{System identification techniques compared to posturography}

System identification techniques are a new engineering approach to assess standing balance. In contrast with posturography, a general used technique to assess standing balance, it is possible to detect underlying systems and used strategies in standing balance (Engelhart et al., 2014; Pasma et al., 2014b). In this study, we assessed standing balance with both system identification techniques and posturography (i.e. body sway). Compared to system identification techniques, no systematic errors and a higher reliability were found for posturography.

In comparison with our results of system identification techniques, studies investigating the reliability of the Sensory Organization Test (SOT) showed a learning effect in healthy young due to changes in postural strategies or through reweighting of sensory information. Remarkably, this learning effect was only present in more demanding test conditions (Wrisley et al., 2007). Studies investigating the reliability of Center of Pressure (CoP) parameters did not find systematic errors (Ageberg et al., 2003; Moghadam et al., 2011), which is comparable with our results of the body sway, but in contrast with the system identification techniques results. This could be explained by the influence of used strategies to maintain balance on the parameters. CoP parameters only describe objectively standing balance, while system identification techniques also describe the underlying changes. Therefore, changes in strategies between trials will not be detected by CoP parameters and do not influence the reliability of CoP parameters.

The reliability of the SOT was moderate in noninstitutionalized old adults when 2 sessions of the test were performed 1 week apart. To improve the reliability of the computer-generated scores of the SOT, a modification of the scoring system was recommended (Ford-Smith et al., 1995). The reliability of CoP parameters depends on the test condition, study design, study population and therapeutic interventions (Ruhe et al., 2010). To reach an excellent reliability of CoP parameters, the duration of the trial must be minimal 90 seconds, must by three to five times repeated and must be measured with eyes closed and on a firm surface (Ruhe et al., 2010). Santos et al. (2007) showed that at least 7 repetitions must be performed to reach an excellent reliability for CoP parameters (Santos et al., 2008). This is comparable with our study, in which measurements of approximately two minutes were used to assess standing balance and must be repeated ten times to reach an excellent reliability. The found relative low SEM\% $(<20 \%)$ are comparable with other studies using CoP parameters (Santos et al., 2008). 


\section{Clinical recommendations}

First, the results indicated that there is a systematic error between the first trial and the second trial. This could be due to changes in used strategies to maintain standing balance and time needed to reach a steady state. Therefore, to assess steady state balance we recommend to perform one familiarization trial on each day. As in this study only two trials were performed per day, it was not possible to assess the number of trials needed to reach an excellent reliability when omitting the first trial from analysis. Second, results showed that averaging over days is more effective than averaging within days. However, in clinical practice it is not feasible to measure on more than one day. Performing multiple measurements on one day could be hampered by fatigue or boredom of the participant, which has to be taken into account.

As mentioned before, systematic errors might be due to more time needed for reaching a steady state balance or a redundancy of applied strategies. This implies that parameters obtained with system identification techniques are sensitive for detection of adaptation strategies. Besides steady state balance, adaptation strategy and adaptation time may have clinical meaning and need further exploration. System identification techniques are sensitive tools to assess the duration of adaptation of sensory reweighting (Asslander and Peterka, 2014) in contrast to e.g. CoP measurement.

\section{Strengths and limitations}

The strength of this study is the selection of healthy old participants, resulting in a well phenotyped group. However, this also affects ICC and ID. Low variability within the participants (i.e. a homogeneous population) results in lower ICC and ID values and therefore lower relative reliabilities (Keating and Matyas, 1998; Weir, 2005). SEM(\%) and MDC are measures of absolute reliability and important measures when interpreting results of repeated measures effects of intervention. Another strength of this study is the set up with exactly one week between sessions. A limitation of this study is the relative low number of participants. As only two trials per day were performed, reliability within a day could not be assessed excluding the first (familiarization) trial.

\subsection{Conclusion}

This study investigated the reliability of a comprehensive set of parameters obtained with system identification techniques to assess standing balance in a population of healthy elderly. Systematic errors were present between trials showing sensitivity of parameters obtained with system identification techniques for detection of adaptation strategies. To assess steady state balance a training session is recommended. As only a single trial per day resulted in poor to moderate reliability, it is recommended to perform more trials on separate days. Within the present framework, acceptable reliability of steady state balance assessment could be achieved by measuring and averaging at least ten trials on the same day. 


\section{Chapter 7}

\section{General Discussion}

Balance control involves the contribution of neural, muscular and sensory systems, which work together via complex feedback pathways in a closed loop. With age or disease, the underlying systems in balance control can deteriorate; e.g. muscle strength decreases, the sensory systems become less accurate, the processing time of sensory information increases and the neural conduction time increases (Rubenstein, 2006; Sturnieks et al., 2008). To maintain balance in various situations and to prevent falling, the underlying systems can compensate for each other's deterioration; i.e. there exists some redundancy within the closed loop system of balance control. However, when the deterioration in the underlying system is too severe, or when the systems which could compensate are also affected, impaired balance tends to become symptomatic and the risk of falling increases (Muir et al., 2010). In Europe, approximately 30\% of people over 65 years of age fall each year. Between $20 \%$ and $30 \%$ of those who fall suffer from injuries that reduce mobility and independence - such as hip fractures (1-2\%), other fractures (3-5\%) or head injuries (5\%) (NVGK, 2004) - and increase the risk of premature death (WHO, 2007).

This thesis contributes to the large societal problem of balance impairment and associated falls. The general goal was to create further insight in the (patho-)physiology of standing balance control in elderly. To prescribe targeted therapy on an individual level to reduce the consequences of falls (Engelhart et al., 2014; Pasma et al., 2014b), it is first important to detect the primarily deteriorated underlying system and the possible compensation strategies that are at work. In this thesis, a novel experimental set-up and data-analysis method was introduced to assess the contribution of the underlying mechanisms in standing balance control. With this experimental approach age-related changes in standing balance control were quantified and the approach for application in clinical practice was validated. In this discussion, the major objectives of the thesis are summarized and discussed. Furthermore, some implications and directions for future research are described together with a long term perspective of balance control studies and its clinical application. 


\subsection{Assessing the contribution of the underlying mechanisms in standing balance control}

If one investigates balance control and ignores the closed-loop property of balance control, cause and effect relations remain unclear and the contribution and quality of the underlying systems are almost impossible to detect, especially when someone uses compensation strategies. Chapter 2 describes that clinical balance tests are unable to reveal how well the underlying systems in balance control are functioning, they have little influence on clinical decision making and prescribing targeted therapy to reduce the consequences of impaired balance (Visser et al., 2008; Pasma et al., 2014b). Therefore, the first research objective of the thesis was to develop novel and improved experimental techniques to assess the contribution of the underlying mechanisms in standing balance control. The neuromuscular controller describes the continuous and closed-loop mechanism of how balance is regulated, and was the system of main interest throughout this thesis. A novel control-engineering approach was introduced, termed system identification techniques. In combination with a specifically designed experimental set-up; i.e. the BalRoom, it became possible to identify the dynamics of the neuromuscular controller, which gives the opportunity to detect changes in balance control with age or disease.

\section{System identification techniques to unravel balance control}

As the field of system identification is very broad there are differences in experimental design, use of disturbance signals and the applied methods. Therefore, in Chapter 3, various existing system identification methods were compared, based on model simulations. Model simulations have the advantage that the outcome of various methods can easily be compared to the known theoretical dynamics of the simulated system. Results indicated that non-parametric system identification techniques estimated the dynamics of the neuromuscular controller reliably, whereas parametric system identification techniques in general resulted in a poor estimation. A disadvantage of parametric methods is that a model structure is assumed to describe the data and the noise properties that influence the system. If the underlying dynamics of the system or noise cannot be described by this model structure, this yields erroneous results. Non-parametric methods estimate the system dynamics without any a priori assumptions about the underlying structure. The external disturbance signals are best chosen to be multisine signals, in favour over noise disturbances, as this resulted in the lowest Noise to Signal Ratio and specific frequencies of interest can be excited. The outcomes of the study in Chapter 3 resulted in an applicable system identification technique with which it became possible to reliably identify the underlying mechanisms (i.e. the neuromuscular controller dynamics) in standing balance control.

Therefore, in the following chapters of this thesis, non-parametric system identification techniques in combination with multisine disturbances were used to identify the dynamics of the neuromuscular controller, which was expressed in Frequency Response Functions (FRFs). Results in Chapter 4-6 show that the FRFs reliably describe the dynamics of the 
neuromuscular controller, and are sensitive enough to detect changes with age or condition; e.g. humans adapt their postural responses to external force fields (Chapter 4), elderly adapt their postural responses less to external force fields compared to young (Chapter 5), and with an increasing disturbance of proprioceptive information, humans lower their reliance on proprioceptive information and increase their reliance on visual information (Chapter 6).

To reveal which mechanisms are underlying the neuromuscular controller dynamics, a parametric model was introduced in Chapter 5, which was fitted onto the obtained FRFs. The outcome measures of the model fits are now specific parameters which allows physiological interpretation. A prerequisite for the reliable estimation of model parameters is a reliable FRF (i.e. obtained non-parametrically with multisine disturbances). Nevertheless, compared to the parametric-system identification methods, interpretation of the model parameters must be done carefully. It is important to keep in mind that a balance must be found between the amount of parameters and the goodness of the model fit. Few parameters result in poorer fits, however the variance within the parameters is lower and therefore the estimated parameters are more accurate. Vice versa, a large amount of parameters improves the goodness of the model fit, but can result in a less accurate estimation due to higher variance within the parameters; i.e. some of the parameters may not provide interpretable information about the system. With the model fits, age related differences in intrinsic properties and time delays were found; i.e. elderly had higher intrinsic stiffness of the ankle joint and larger time delays of the hip, compared to young participants. The parameter values corresponded to other studies, which used similar model fitting approaches (Peterka, 2002; Mahboobin et al., 2005; Cenciarini, 2010; Davidson et al., 2011).

In Chapter 6, model parameter estimation on the data obtained with the BalRoom, required the development of extended parametric models, including multi-joint feedback paths and integration of sensory information (Peterka, 2002; Pasma et al., 2015). The best model with the right amount of parameters has yet to be investigated. Therefore only non-parametric parameters which describe balance control were used, such as the average magnitude at low frequencies and the phase shift on high frequencies.

\section{The BalRoom creates the possibility to investigate multivariate balance control}

System identification approaches requires dedicated experiments, with specifically designed equipment to apply external disturbances on specific places of the human body. In Chapter 4, the Double Inverted Pendulum Perturbator (DIPP) was developed to apply well known force disturbances at the level of the hip and between the shoulder blades, which made it possible to study the contribution of the ankles and hips in balance control; i.e. multi-joint coordination. The application of external force fields (implemented as virtual springs in the controller) allowed to study adaptation of multi-joint coordination, by external (de)stabilization of the participants' balance. 
All young and elderly participants were able to maintain balance in all experimental conditions, using equal disturbance amplitudes. The ability to maintain balance must be further studied in a population with impaired balance; e.g. Parkinson's disease patients, which are known to have asymmetrical balance responses (Boonstra et al., 2014a). The amplitude of the disturbances must be low enough to be able to maintain standing balance (this requires submaximal disturbance amplitudes), but high enough to detect a response and to apply system identification techniques.

To obtain more insight in the use of sensory information, two sensory disturbances were added to the force disturbances in Chapter 6. A proprioceptive disturbance was applied with the Bilateral Ankle Perturbator (BAP), which created support surface rotations around the ankle joints. A visual disturbance was applied by rotation of the visual scene, using the VIsual Perturbator (VIP). The integration of all three devices resulted in the BalRoom, in which the two force disturbances and the two sensory disturbances were applied simultaneously (Figure 7.1). This protocol was tested in a population of healthy elderly. All healthy elderly were able to maintain standing balance in all conditions. However, compared to application of only force disturbances in Chapter 5, the amplitude of the disturbances in the BalRoom protocol was lower. Adding multiple disturbances, reduces the amplitude someone can withstand while keeping their feet in place. Nevertheless, the disturbance amplitudes were sufficient to detect responses and to apply system identification techniques.

The BalRoom is a unique device, able to apply multiple disturbances to challenge the balance control system. As the disturbances have separate frequency content, the human responses can be related to each disturbance. Within one measurement, the neuromuscular controller properties can be studied, separating the contribution of proprioceptive information, visual information and the control of the ankle and hip joint. The DIPP was validated in a study detecting age related differences in multi-joint coordination (Chapter 5). The BAP was validated prior to the BalRoom project, in a study detecting changes in sensory weighting and reweighting of proprioceptive information between the left and right leg (Pasma et al., 2012) and with age and disease (Pasma et al., 2015). The BalRoom was validated so far based on a population of healthy elderly (Chapter 6). More evidence is needed for using the BalRoom, based on a comparison between young and healthy elderly and an elderly population with a specific disease or with impaired balance, which is eleborated on further in this discussion.

\subsection{Quantifying age related changes in balance control}

The second research objective of the thesis was to quantify age related changes in balance control. Recent studies have shown that differences between young and elderly were the most pronounced in multi-segmental balance control coordination (Accornero et al., 1997; Hsu et al., 2013); i.e. involving the contribution of the ankles and hips in balance control. Within the clinical practice and research field, various approaches exist to detect balance control differences with age. An easy approach is to use questionnaires about fall history 
and balance impairments. However, the self-reported problems with balance control are often biased, as people tend to forget when they have fallen or under-estimate the risk of impaired balance (Bean et al., 2011). Another recent study showed that the ability to maintain standing balance in side-by-side stance with eyes closed is a useful clinical measure to detect impaired standing balance in elderly (Pasma et al., 2014a). This method is a very easily applicable clinical measure, as it does not require any equipment and it is not time consuming.

Other clinical balance tests are used to measure the quality of standing balance in clinical practice, often looking at Centre of Pressure movements (CoP) derived from standing under various conditions on a force plate (Winter et al., 1990; Visser et al., 2008; Ruhe et al., 2010; Kingma et al., 2011; Pasma et al., 2014a). The CoP movement describes the quality of balance control by representing the quality of all systems together, which hampers the interpretation of CoP movements as cause and effect relations remain unclear. The ability to maintain balance is influenced by various factors; e.g. muscle characteristics, cognition, blood pressure regulation and the quality of the sensory systems. Deterioration in one system can be compensated by one of the other systems and it is therefore difficult to draw conclusions about the primary impaired system.

Age related changes in balance control might become more apparent when the difficulty of the test condition is increased. With increasing difficulty of the condition, the balance control system fails at some point, depending on the quality of the underlying systems and the use of compensation mechanisms. It might be a useful measure to therefore evaluate compensatory stepping responses. A larger number of steps to recover from a perturbation identifies people with impaired balance, which are more prone to falls (Nonnekes et al., 2013). Other age related differences are delayed reaction times, smaller step lengths, lower step velocities and lower joint torques of the stepping leg after foot contact (Weerdesteyn et al., 2012). Impaired balance control can also become apparent during gait. Gait characteristics extracted from trunk acceleration during daily-life gait are associated with fall history and can be complementary to current clinical tests, such as questionnaires (Rispens et al., 2015). Accelerometer measurements during daily life activities have the advantage that laboratory-based gait and balance assessments can be reduced.

Although these techniques are easy to apply in clinical practice, they lack in acquiring information about the deterioration of the underlying systems involved in balance control and they often do not include multi-joint coordination (Chapter 2). System identification techniques allow to study age related changes in balance control, detecting the contribution and quality of the underlying mechanisms. 


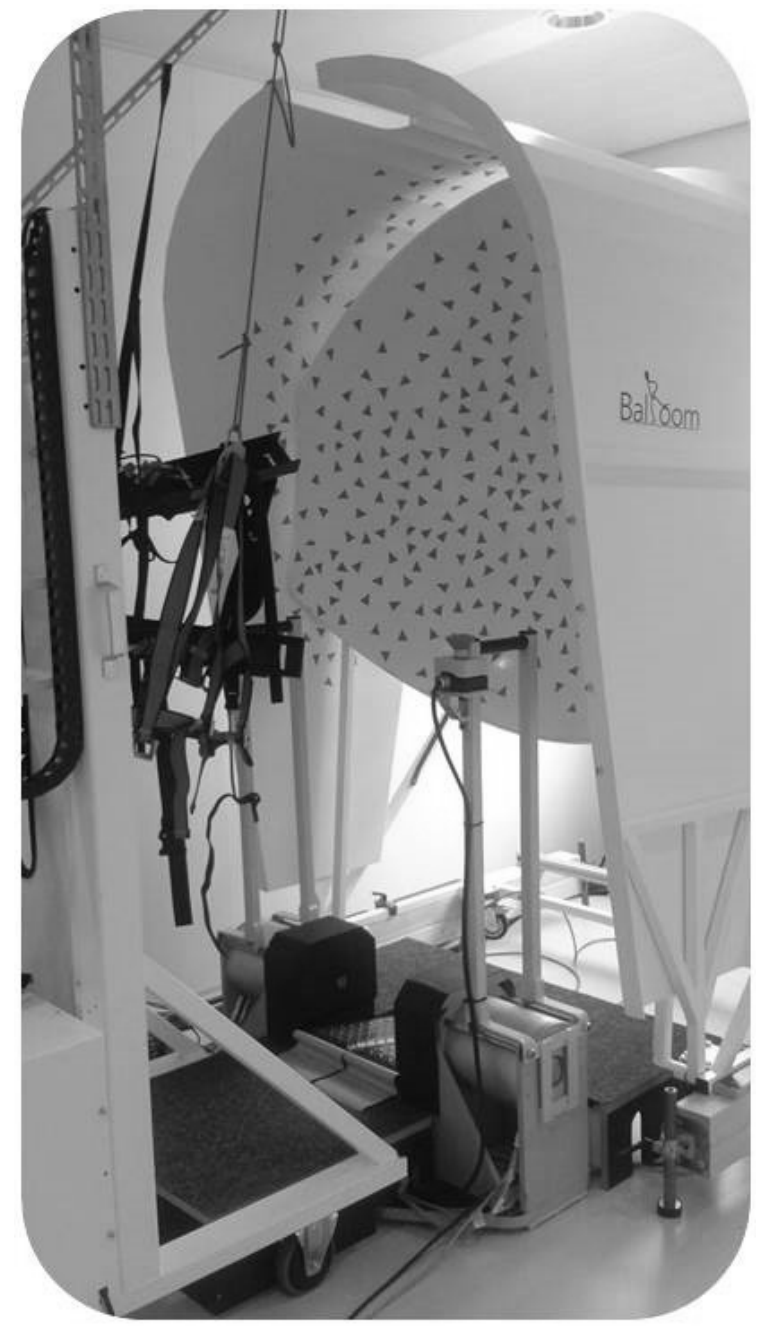

The Balance Test Room (BalRoom)

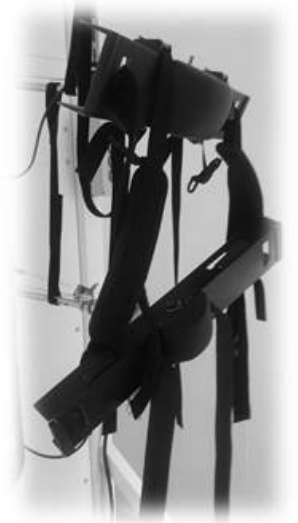

The Double Inverted Pendulum Perturbator (DIPP)

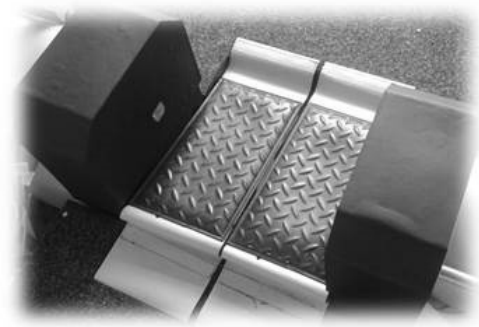

The Bilateral Ankle Perturbator (BAP)

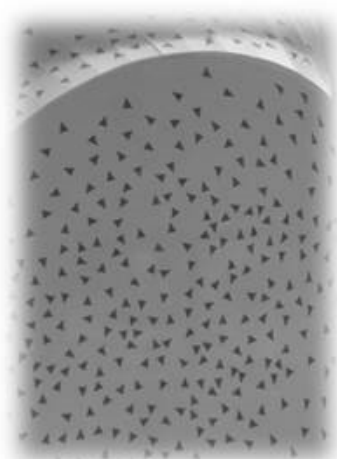

The Visual Perturbator (VIP)

Figure 7.1: Experimental setup of the BalRoom, consisting of three devices; the DIPP, BAP and VIP. The BalRoom makes it possible to identify the contribution of the ankles and hips in balance control, together with the contribution of proprioceptive and visual information. 
In Chapter 5, system identification techniques were applied to investigate how humans adapt their multi-joint coordination to adjust to external (de)stabilizing force fields and whether this differed with age. Results showed that elderly generated similar corrective joint torques but had reduced body sway compared to young participants; suggesting that elderly were stiffer. When a stabilizing or destabilizing external force field was applied at the hip, both young and elderly participants adapted their multi-joint coordination by lowering or respectively increasing their neuromuscular control actions around the ankles; expressed in a change of FRF magnitude. However, elderly adapted less compared to young participants. Model fits on the FRFs showed that elderly had comparable reflexive stiffness and reflexive damping to the young. However, elderly had higher intrinsic stiffness properties of the ankles their time delays around the hip were increased. With the current clinical balance tests, it was not possible to distinguish cause and effect relations in a closed loop and the contribution of the underlying systems involved in standing balance. With the system identification approach it now became possible to quantify age-related changes in balance control, including differences in multi-joint coordination.

The age related differences in Chapter 5 result from a population of healthy elderly. More evidence is needed by detecting changes in elderly with a known deterioration in balance control. Another study performed within the BalRoom project shows that with the Bilateral Ankle Perturbator (BAP), it is possible to distinguish underlying changes in the use of proprioceptive information within an elderly population with cataract, polyneuropathy and impaired balance (Pasma et al., 2015). Another study using system identification approach shows that it possible to detect asymmetries in balance control, in a population of Parkinson's' disease patients and age matched controls (Boonstra et al., 2014a). The ultimate proof to validated the use of system identification techniques to detect age related changes in balance control, is to select a random subject from a healthy young population, a healthy elderly population, and a population with a known deterioration of a specific underlying system and trace from which group this subject was selected. Clustering of phenotypes allows to study the sensitivity of system identification methods to detect specific deteriorations in balance control.

\subsection{The application of system identification techniques in a clinical setting}

The third and final research objective of the thesis was to validate the use of system identification techniques for clinical application. Before introducing the method into clinical practice for diagnosing or monitoring treatment of impaired balance, it is important to assess the reliability of this technique and compare it with current clinical balance measures. In Chapter 6, the reliability of standing balance parameters was studied, with all BalRoom disturbances applied simultaneously (i.e. two force disturbances at the hip and shoulder and two sensory disturbances of proprioceptive and visual information) and using system identification techniques to assess standing balance in healthy elderly. The balance parameters were non-parametrically obtained values from the FRF, representing the mean 
magnitude at low frequencies and the phase shift at high frequencies. The reliability was compared with a parameter that represented the amount of body sway, often looked at in clinical balance tests. Results were obtained by measuring balance parameters twice during three days.

A systematic error was found in the balance parameters; i.e. parameters differed between the first and second trial on one day. Therefore, when steady state balance control is to be assessed with system identification techniques, a familiarization trial is needed on the same day to overcome the systematic error between trials. Although the parameters were reliable; i.e. changes over time could be detected within the same participant (e.g. effects of intervention or changes in conditions), results showed that at least 10 trials on one day were needed to reach an excellent reliability for steady state balance assessment. As one trial lasted approximately 2 minutes, 20 minutes of data were required to assess balance control within one experimental condition. When measuring on multiple days, less trials per day were needed to reach an excellent reliability.

Aiming for excellent reliability poses restrictions on clinical application. There is limited time in clinical practice and it therefore preferable to perform all measurement trial on the same day. In Chapter 6, we studied sensory reweighting of proprioceptive information in three experimental conditions (support surface rotations). To reach an excellent reliability, approximately 60 minutes of data must be recorded. Fatigue and boredom of the participants may now play an important role, which hampers clinical implementation.

Compared to system identification techniques, no systematic errors and a higher reliability were found for the body sway parameter. However, the body sway parameters only describes standing balance objectively, while system identification techniques also describe the underlying changes. Therefore, the use of system identification techniques has added value over current clinical balance measures, such as body sway. Possibly, the best of two worlds can be combined; body sway measures can first detect impaired balance control, and with the use of system identification techniques the underlying cause can be detected.

\subsection{Future directions}

\section{Towards targeted interventions of impaired balance}

In future studies it is important to expand the reliability studies from FRFs to estimated model parameters. This might indicate the accuracy of the model structure of the neuromuscular controller and the minimal detectable change of the parameters. Furthermore, a next step is to relate the estimated parameters to treatment options, to validate if targeted interventions can be set up based on the outcomes of the system identification techniques. So far, the BalRoom and system identification techniques give more insight in the underlying physiology of balance control and the changes with age. 


\section{Development of time-varying system identification techniques}

The system identification methods used throughout this thesis, assume that underlying dynamics of the neuromuscular controller behaves linearly and time invariant (the behaviour is not altered over time). With the right experiment and small disturbance amplitudes, human stance behaviour can be assumed linear around the point of equilibrium (upright stance) and with short measurement times (which reduces fatigue), time invariant behaviour can be assumed. Therefore the LTI (linear and time-invariant) system identification techniques, as were used in this thesis give reliable estimates of the neuromuscular controller dynamics.

However, the parts that compose the neuro-musculoskeletal system show a time-varying behaviour, as changing muscle activation is required for various tasks (de Vlugt et al., 2002), (Ludvig et al., 2011). Furthermore, postural responses typically adapt or habituate when perturbations have various directions and sizes (Keshner et al., 1987; Bloem et al., 1998). Finally, there are changes due to performance limitations, like fatigue. In sum, humans adapt their behaviour in time, indicating that balance control is anticipative, which challenges the assumptions of linearity and time invariance. With age or disease, a slower adaptation to changing environmental conditions increases the risk of falling.

Experimental studies also report on this time-varying behaviour. One study (Goodworth and Peterka, 2012) found that subjects effectively adapt their sway response in a changing environment, by down-weighting the unreliable sensory information and up-weighting the other information sources. This down-weighting occurs faster than up-weighting as unreliable information is more threatening for balance control and therefore adaptability of sensory reweighting is of interest. The speed of reweighting is functionally important because failure to adjust rapidly enough can result in instability and falls when subjects either fail to generate sufficient corrective torque to resist gravity or generate too much torque resulting in resonant behaviour (Asslander and Peterka, 2014). Another study (van Asseldonk et al., 2006) shows that in stroke patients, the contribution in balance control between the two legs can be asymmetrical. Posing more weight on the affected leg can alter the controller properties between the legs to remain an upright stance.

These studies showed variation in control behaviour between changing environments or operation points, when the experimental outcomes are averaged over multiple trails. Nevertheless, the response to very first and fully unpracticed trials may provide useful insights into the mechanisms associated with truly unexpected adaptations. We thereby gain more understanding about the underlying mechanisms in human balance control, compared to actions that happen in everyday tasks. To study this variation in time and to identify the changes in human balance control behaviour, time-varying system identification techniques are needed. As the current LTI techniques as were used in this thesis, requires an average over ten trials to obtain excellent reliability, detecting time varying behaviour is challenging. It possibly requires many transitions between conditions, increasing measurement time and fatigue of the participants. 


\section{Future perspectives of the BalRoom}

In addition to reliable system identification methods, accurate application of external disturbances is important to obtain an estimate of the neuromuscular controller dynamics. The Balance Test Room (BalRoom) was developed modularly, to test if the application of individual disturbances resulted in the desired outcome measures. Subsequently, the modules were combined to study the application of simultaneous disturbances on the balance control system. The BalRoom device as it exists now poses restrictions on the clinical applicability. Although the BalRoom is a stand-alone device, the integration of the equipment can be improved. The BalRoom is extremely large and cannot easily be transferred between rooms within or between clinics. Further development should focus on integration of the three modules, reducing the size and making it transferable at an affordable price. Additionally to the existing hardware, it is recommended to include electromyography (EMG) measurements to obtain a reliable estimate of the contraction level of the muscles (in addition to measuring joint torques). Furthermore, one can include galvanic stimulation to study the quality and integration of all three sensory systems. Finally, adding a cognitive task can expand the current BalRoom to include cognitive influences on balance control.

Using the BalRoom and system identification techniques as a diagnostic tool, can help to detect impaired balance and ultimately reduce the consequences of falls in the elderly. 


\section{Bibliography}

Abrahamová D, Hlavačka F. Age-Related Changes of Human Balance during Quiet Stance. Physiol Res 57: 957-964, 2008.

Accornero N, Capozza M, Rinalduzzi S, Manfredi GW. Clinical multisegmental posturography: age-related changes in stance control. Electroencephalogr Clin Neurophysiol 105: 213-219, 1997.

Ageberg E, Roberts D, Holmstrom E, Friden T. Balance in single-limb stance in healthy subjects--reliability of testing procedure and the effect of short-duration sub-maximal cycling. BMC Musculoskelet Disord June 27: 4:14, 2003.

Alexandrov A V, Frolov A., Massion J. Biomechanical analysis of movement strategies in human forward trunk bending. I. Modeling. Biol Cybern 84: 425-434, 2001a.

Alexandrov A V, Frolov AA, Horak FB, Carlson-Kuhta P, Park S. Feedback equilibrium control during human standing. Biol Cybern 93: 309-322, 2005.

Alexandrov A V, Frolov AA, Massion J. Biomechanical analysis of movement strategies in human forward trunk bending. II. Experimental study. Biol Cybern 84: 435-443, 2001b.

Ambrose AF, Paul G, Hausdorff JM. Risk factors for falls among older adults: A review of literature. Maturitas 75: 51-61, 2013.

Amiridis IG, Hatzitaki V, Arabatzi F. Age-induced modifications of static postural control in humans. Neurosci Lett 350: 137-140, 2003.

Anon NeurCom [Online]. 2011.

http://www.resourcesonbalance.com/neurocom/protocols/sensoryImpairment/SOT.aspx.

Van Asseldonk EHF, Buurke JH, Bloem BR, Renzenbrink GJ, Nene A V, van der Helm FCT, van der Kooij H. Disentangling the contribution of the paretic and non-paretic ankle to balance control in stroke patients. Exp Neuro/ 201: 441-51, 2006.

Van Asseldonk EHF, Carpenter MG, van der Helm FCT, van der Kooij H. Use of induced acceleration to quantify the (de)stabilization effect of external and internal forces on postural responses. [Online]. IEEE Trans Biomed Eng 54: 2284-95, 2007.

http://www.ncbi.nlm.nih.gov/pubmed/18075045. 
Van Asseldonk EHF, Wessels M, Stienen AH, van der Helm FCT, van der Kooij H. Influence of haptic guidance in learning a novel visuomotor task. J Physiol - Paris 103: 276285, 2009.

Asslander L, Peterka RJ. Sensory reweighting dynamics in human postural control. J Neurophysiol 111: 1852-1864, 2014.

Barin K. Evaluation of a generalized model of human postural dynamics and control in the sagittal plane. Biol Cybern 61: 37-50, 1989.

Bean JF, Olveczky DD, Kiely DK, LaRose SI, Jette AM. Performance-based versus patient-reported physical function: what are the underlying predictors? Phys Ther 91: 1804$1811,2011$.

Benjuya N, Melzer I, Kaplanski J. Aging-induced shifts from a reliance on sensory input to muscle cocontraction during balanced standing. J Gerontol A Biol Sci Med Sci 59: 166$171,2004$.

Berg K, Wood-Dauphinee S, Williams JI, Gayton D. Measuring balance in the elderly: preliminary development of an instrument. Physiother Canada 41: 304-11, 1989.

Bijlsma AY, Pasma JH, Lambers D, Stijntjes M, Blauw GJ, Meskers CGM, Maier AB. Muscle strength rather than muscle mass is associated with standing balance in elderly outpatients. J Am Med Dir Assoc In press, 2013.

Bloem BR, Steijns JAG, Smits-Engelsman BC. An update on falls. Curr Opin Neuro/ 16: 15-26, 2003.

Bloem BR, van Vugt J, Beckley D, Remler M, Roos R. Habituation of lower leg stretch responses in Parkinson's Disease. Electroencephalogr Clin Neurophysiol 109: 73-7, 1998.

Boonstra TA, Schouten AC, van der Helm FCT, van der Kooij H. Identification of the contribution of the ankle and hip joints to multi-segmental balance control. J Neural Eng Rehabil 10: 23, 2013.

Boonstra TA, Schouten AC, van Vugt JPP, Bloem BR, van der Kooij H. Parkinson's disease patients compensate for balance control asymmetry. J. Neurophysiol. .

Boonstra TA, van Vugt JPP, van der Kooij H, Bloem BR. Balance asymmetry in Parkinson's disease and its contribution to freezing of gait. PLoS One 9: e102493, 2014b.

Bronstein AM, Brandt T, Woollacott M. Clinical Disorders of Balance, Posture and Gait. London: Arnold, 1996.

Burdet E, Osu R, Franklin DW, Milner TE, Kawato M. The central nervous system stabilizes unstable dynamics by learning optimal impedance. Nature 414: 446-9, 2001. 
Cenciarini M, Peterka RJ. Stimulus-dependent changes in the vestibular contribution to human postural control. J Neurophysio/ 95: 2733-50, 2006.

Cenciarini M. Stiffness and damping in postural control increase with age. IEEE Trans Biomed Eng 57: 267-275, 2010.

Cohen H, Heaton LG, Congdon SL, Jenkins HA. Changes in sensory organization test scores with age. Age Aging 25: 39-44, 1996.

Creath R, Kiemel T, Horak F, Peterka R, Jeka J. A unified view of quiet and perturbed stance: simultaneous co-existing excitable modes. Neurosci Lett: 75-80, 2005.

Cummings S., Kelsey J., Nevitt M., O’Dowd K. Epidemiology of osteoporosis and osteoporotic fractures. Epidemiol Rev 7: 178-208, 1985.

Cummings S., Nevitt M., Kidd S. Forgetting falls. The limited accuracy of recall of falls in the elderly. J Am Geriatr Soc 36: 613-616, 1988.

Davidson BS, Madigan ML, Southward SC, Nussbaum MA. Neural Control of Posture During Small Magnitude Perturbations: Effects of Aging and Localized Muscle Fatigue. 58: 1546-1554, 2011.

Demura S-I, Kitabayashi T, Aoki H. Body-sway characteristics during a static upright posture in the elderly. Geriatr Gerontol Int 8: 188-197, 2008.

Doumas M, Krampe RT. Adaptation and reintegration of proprioceptive information in young and older adults' postural control. J Neurophysio/ 104: 1969-77, 2010.

Doumas M, Rapp M a, Krampe RT. Working memory and postural control: adult age differences in potential for improvement, task priority, and dual tasking. J Gerontol $B$ Psychol Sci Soc Sci 64: 193-201, 2009.

Engelhart D, Pasma JH, Schouten AC, Meskers CGM, Maier AB, Mergner T, van der Kooij H. Impaired standing balance in elderly: a new engineering method helps to unravel causes and effects. J Am Med Dir Assoc 15: 227.e1-6, 2014.

Engelhart D, Schouten AC, Aarts RGKM, Kooij H Van Der. Assessment of Multi-Joint Coordination and Adaptation in Standing Balance: a Novel Device and System Identification Technique. IEEE Trans. Neural Syst. Rehabil. Eng. doi: DOI 10.1109.

Fitzpatrick R, Burke D, Gandevia SC. Loop gain of reflexes controlling human standing measured with the use of postural and vestibular disturbances. J Neurophysiol 76: 39944008, 1996.

Fleiss RL. The design and analysis of clinical experiments. 1986. 
Folstein M, Folstein S, McHugh PR. "Mini-mental state". A practical method for grading the cognitive state of patients for the clinician. J psychiat Res 12: 189-198, 1975.

Ford-Smith CD, Wyman JF, Elswick RKJ, Fernandez T, Newton RA. Test-retest reliability of the sensory organization test in noninstitutionalized older adults. Arch Phys Med Rehabil 76: 77-81, 1995.

Franklin DW, Osu R, Burdet E, Kawato M, Milner TE. Adaptation to stable and unstable dynamics achieved by combined impedance control and inverse dynamics model. $J$ Neurophysio/90: 3270-3282, 2003.

Fujisawa N, Masuda T, Inaoka H, Fukuoka Y, Ishida A, Minamitani H. Human standing posture control system depending on adopted strategies. Med Biol Eng Comput 43: 107-114, 2005.

Ganz DA, Bao Y, Shekelle PG, Rubenstein LZ. Will My Patient Fall? JAMA 297: 77-86, 2007.

Gariépy C, Hasson CJ, Emmerik REA Van, Caldwell GE. Age related decrease in degrees of freedom in postural control during quiet stance. In: 16th ESB Congress. 2008.

Geurts ACH, Boonstra TA, Voermans NC, Diender MG, Weerdesteyn V, Bloem BR. Assessment of postural asymmetry in mild to moderate Parkinson 's disease. Gait Posture 33: 143-145, 2011.

Goodworth AD, Peterka RJ. Sensorimotor integration for multisegmental frontal plane balance control in humans. J Neurophysiol 107: 12-28, 2012.

Guralnik JM, Simonsick EM, Ferrucci L, Glynn RJ, Berkman LF, Blazer DG, Scherr PA, Wallace RB. A short physical performance battery assessing lower extremity function: association with self-reported disability and prediction of mortality and nursing home admission. J Gerontol 49: M85-M94, 1994.

Hendershot BD, Bazrgari B, Nussbaum MA, Madigan ML. Within- and between-day reliability of trunk mechanical behaviors estimated using position-controlled perturbations. $J$ Biomech 45: 2019-2022, 2012.

Henry SM, Fung J, Horak FB, Goodworth AD, Mellodge P, Peterka RJ, Honeycutt CF, Nichols TR, Horak FAYB. EMG Responses to Maintain Stance During Multidirectional Surface Translations EMG Responses to Maintain Stance During Multidirectional Surface Translations. J Neurophysio/ 80: 1939-1950, 1998.

Horak FB, Nashner LM. Central programming of postural movements. J Neurophysio/55: 1369-1381, 1986.

Horak FB, Shupert CL, Mirka A. Components of postural dyscontrol in the elderly: a review. Neurobiol Aging 10: 727-738, 1989. 
Horak FB. Postural orientation and equilibrium: what do we need to know about neural control of balance to prevent falls? Age Ageing 35 Suppl 2: ii7-ii11, 2006.

Hsu W-L, Chou L-S, Woollacott M. Age-related changes in joint coordination during balance recovery. Age (Omaha) 35: 1299-1309, 2013.

Hsu W-L, Scholz JP, Schöner G, Jeka JJ, Kiemel T. Control and estimation of posture during quiet stance depends on multijoint coordination. J Neurophysio/97: 3024-3035, 2007.

Ishida A, Masuda T, Inaoka H, Fukuoka Y. Stability of the human upright stance depending on the frequency of external disturbances. Med Biol Eng Comput 46: 213-221, 2008.

Jeka J, Allison L, Saffer M, Zhang Y, Carver S, Kiemel T. Sensory reweighting with translational visual stimuli in young and elderly adults: the role of state-dependent noise. Exp Brain Res 174: 517-27, 2006.

Jeka JJ, Allison LK, Kiemel T. The dynamics of visual reweighting in healthy and fallprone older adults. J Mot Behav 42: 197-208, 2010.

Johansson R, Magnusson M, Fransson P a, Karlberg M. Multi-stimulus multi-response posturography. Math Biosci 174: 41-59, 2001.

Jonsson R, Sixt E, Landahl S, Rosenhall U. Prevalence of dizziness and vertigo in an urban elderly population. J Vestib Res 14: 47-52, 2004.

Kearney RE, Stein RB, Parameswaran L. Identification of intrinsic and reflex contributions to human ankle stiffness dynamics. IEEE Trans Biomed Eng 44: 493-504, 1997.

Keating J, Matyas T. Unreliable inferences from reliable measurements. Aust J Physiolther 44: 5-10, 1998.

Keshner E, Allum J, Pfaltz C. Postural coactivation and adaptation in the sway stabilizing responses of normals and patients with bilateral vestibular deficit. Exp Brain Res 69: 77-92, 1987.

Kiemel T, Elahi AJ, Jeka JJ. Identification of the plant for upright stance in humans: multiple movement patterns from a single neural strategy. J Neurophysiol 100: 3394-3406, 2008.

Kiemel T, Zhang Y, Jeka JJ. Identification of neural feedback for upright stance in humans: stabilization rather than sway minimization. J Neurosci 31: 15144-15153, 2011.

Kim S, Atkeson CG, Park S. Perturbation dependent selection of postural feedback gain and its scaling. J Biomech 45: 1379-86, 2012. 
Kim S, Horak FB, Carlson-Kuhta P, Park S. Postural Feedback Scaling Deficits in Parkinson's Disease. J Neurophysio/ 102: 2910-2920, 2009.

Kingma H, Gauchard GC, de Waele C, van Nechel C, Bisdorff A, Yelnik A, Magnusson M, Perrin PP. Stocktaking on the development of posturography for clinical use. J Vestib Res 21: 117-125, 2011.

Klomp A, Groot JH De, Vlugt E De, Meskers CGM, Arendzen JH, Helm FCT Van Der. Neuromechanical Wrist Joint Properties. 61: 1005-1014, 2014.

Konrad HR, Girardi M, Helfert R. Balance and aging. Laryngoscope 109: 1454-1460, 1999.

Van der Kooij H, van Asseldonk E, van der Helm FCT. Comparison of different methods to identify and quantify balance control. J Neurosci Methods 145: 175-203, 2005.

Van der Kooij H, van Asseldonk EHF, Geelen J, van Vugt JPP, Bloem BR. Detecting asymmetries in balance control with system identification: first experimental results from Parkinson patients. J Neural Transm 114: 1333-1337, 2007.

Van der Kooij H, Peterka RJ. Non-linear stimulus-response behavior of the human stance control system is predicted by optimization of a system with sensory and motor noise. $J$ Comput Neurosci 30: 759-78, 2011.

Van der Kooij H, de Vlugt E. Postural responses evoked by platform pertubations are dominated by continuous feedback. J Neurophysio/98: 730-43, 2007.

Kuo AD, Speers RA, Peterka RJ, Horak FB. Effect of altered sensory conditions on multivariate descriptors of human postural sway. Exp brain Res 122: 185-195, 1998.

Kuo AD. An optimal state estimation model of sensory integration in human postural balance. J Neural Eng 2: 235-249, 2005.

Laessoe U, Hoeck HC, Simonsen O, Sinkjaer T, Voigt M. Fall risk in an active elderly population-can it be assessed? J Negat Results Biomed 6, 2007.

Lariviere C, Ludvig D, Kearney R, Mecheri H, Caron JM, Preuss R. Identification of intrinsic and reflexive contributions to low-back stiffness: medium-term reliability and construct validity. J Biomech 48: 254-261, 2015.

Laughton C, Slavin M, Katdare K, Nolan L, Bean JF, Kerrigan DC, Phillips E, Lipsitz LA, Collings JJ. Aging, muscle activity, and balance control: physiologic changes associated with balance impairment. Gait Posture 18: 101-108, 2003.

Lin HW, Bhattacharyya N. Balance disorders in the elderly: Epidemiology and functional impact. Laryngoscope 122: 1858-1861, 2012. 
Lindeboom J, Schmand B, Tulner L, Walstra G, Jonker C. Visual association test to detect early dementia of the Alzheimer type. J Neurol Neurosurg Phsychiatry 73: 126-133, 2002.

Ljung L. System Identification: Theory for the user. Prentice-Hall, 1999.

Ljung L. Perspectives on system identification. Annu Rev Contro/34: 1-12, 2010.

Ludvig D, Visser TS, Giesbrecht H, Kearney RE. Identification of time-varying intrinsic and reflex joint stiffness. IEEE Trans Biomed Eng 58: 1715-23, 2011.

Mahboobin A, Loughlin P, Atkeson C, Redfern M. A mechanism for sensory reweighting in postural control. Med Biol Eng Comput 47: 921-929, 2009.

Mahboobin A, Loughlin PJ, Redfern MS, Sparto PJ. Sensory re-weighting in human postural control during moving-scene perturbations. Exp brain Res 167: 260-267, 2005.

Mahboobin A. A model-based approach to attention and sensory integration in postural control of older adults. Neurosci Lett 429: 147-151, 2007.

Manchester D, Woollacott M, Zederbauer-Hylton N, Marin O. Visual, vestibular and somatosensory contributions to balance control in the older adult. J Geronto/44: M118M127, 1989.

Mancini M, Horak FB. The relevance of clinical balance assessment tools to differentiate balance deficits. Eur J Phys Rehabil Med 46: 239-248, 2010.

Marieb EN. Human Anatomy and Physiology. San Fransisco: Pearson Education, Inc., 2004.

Marigold DS, Eng JJ. Altered timing of postural reflexes contributes to falling in persons with chronic stroke. Exp brain Res 171: 459-68, 2006.

Mathias S, Nayak U, Isaacs B. Balance in elderly patients: the "get-up and go" test. Arch Phys Med Rehabil 67: 387-9, 1986.

Matthews PBC. The human stretch reflex and the motor cortex. Trends Neurosci 14: 8791, 1991.

Mergner T, Schweigart G, Maurer C, Blümle A. Human postural responses to motion of real and virtual visual environments under different support base conditions. Exp brain Res 167: 535-56, 2005.

Mergner T. A neurological view on reactive human stance control. Annu Rev Contro/34: 177-198, 2010. 
Merlo A, Zemp D, Zanda E, Rocchi S, Meroni F, Tettamanti M, Recchia A, Lucca U, Quadri P. Postural stability and history of falls in cognitively able older adults: The Canton Ticino study. Gait Posture 36: 662-6, 2012.

Moghadam M, Ashayeri H, Salavati M, Sarafzadeh J, Taghipoor KD, Saeedi A, Salehi R. Reliability of center of pressure measures of postural stability in healthy older adults: effects of postural task difficulty and cognitive load. Gait Posture 33: 651-655, 2011.

Muir SW, Berg K, Chesworth B, Klar N, Speechley M. Quantifying the magnitude of risk for balance impairment on falls in community-dwelling older adults: a systematic review and meta-analysis. J Clin Epidemio/63: 389-406, 2010.

Nashner LM. Brain Adapting Reflexes Controlling the Human Posture. Exp Brain Res 72: 59-72, 1976.

Nasreddine Z., Philips N., Bédirian V, Charbonneau S, Whitehead V, Collin I, Cummings J., Chertkow H. The Montreal Cognitive Assessment, MoCA : A Brief Screening. J Am Geriatr Soc 53: 695-699, 2005.

Noël M, Cantin B, Lambert S, Gosselin CM, Bouyer LJ. An electrohydraulic actuated ankle foot orthosis to generate force fields and to test proprioceptive reflexes during human walking. IEEE Trans Neural Syst Rehabil Eng 16: 390-9, 2008.

Nonnekes J, de Kam D, Geurts ACH, Weerdesteyn V, Bloem BR. Unraveling the mechanisms underlying postural instability in Parkinson's disease using dynamic posturography. Expert Rev Neurother 1: 1-6, 2013.

Nutt JG, Horak FB, Bloem BR. Milestones in Gait, Balance and Falling. Mov Disord 26: 1166-1174, 2011.

NVGK. Richtlijn Preventie van valincidenten bij ouderen. Utrecht: Nederlandse Vereniging voor Klinische Geriatrie, 2004.

Ooteghem K Van, Frank JS, Horak FB. Practice-related improvements in posture control differ between young and older adults exposed to continuous, variable amplitude oscillations of the support surface. Exp Brain Res 199: 185-193, 2009.

Panzer VP, Wakefield DB, Hall CB, Wolfson LI. Mobility assessment: sensitivity and specificity of measurement sets in older adults. Arch Phys Med Rehabil 92: 905-12, 2011.

Park S, Horak FB, Kuo AD. Postural feedback responses scale with biomechanical constraints in human standing. Exp brain Res 154: 417-27, 2004.

Pasma JH, Bijlsma AY, van der Bij MDW, Arendzen JH, Meskers CGM, Maier AB. Age-related differences in quality of standing balance using a composite score. Gerontology 60: 306-14, 2014a. 
Pasma JH, Boonstra TA, Campfens SF, Schouten AC, van der Kooij H. Sensory reweighting of proprioceptive information of the left and right leg during human balance control. J Neurophysio/ 108: 1138-48, 2012.

Pasma JH, Engelhart D, Maier AB, Schouten AC, Kooij H van der, Meskers CGM. Changes in sensory weighting and reweighting of proprioceptive information during standing balance with age and disease. Submitted. .

Pasma JH, Engelhart D, Schouten AC, van der Kooij H, Maier AB, Meskers CGM. Impaired standing balance: The clinical need for closing the loop. Neuroscience 267C: 157$165,2014 b$.

Pavol MJ, Runtz EF, Edwards BJ, Pai Y. Age influences the outcome of a slipping perturbation during inital but not repeated expostures. J Geronto/57A: 496-503, 2002.

Perreault EJ, Kirsch RF, Acosta AM. Multiple-input, multiple-output system identification for characterization of limb stiffness dynamics. Biol Cybern 80: 327-337, 1999.

Peterka RJ, Loughlin PJ. Dynamic regulation of sensorimotor integration in human postural control. J Neurophysio/91: 410-423, 2004.

Peterka RJ. Sensimotor integration in human postural control. J Neurophysio/88: 1097$1118,2002$.

Peterka RJ. Simplifying the complexities of maintaining balance. IEEE Eng Med Biol Mag 22: 63-68, 2003.

Pickering RM, Grimbergen YAM, Rigney U, Ashburn A, Mazibrada G, Wood B, Gray P, Kerr G, Bloem BR. A Meta-Analysis of Six Prospective Studies of Falling in Parkinson 's Disease. Mov Disord 22: 1892-1900, 2007.

Pintelon R, Schoukens J. System Identification: $A$ frequency domain approach. John Wiley and Sons, 2012.

Podsiadlo D, Richardson S. The timed "Up\&Go": a test of basic functional mobility for frail elderly persons. J Am Geriatr Soc 39: 142-148, 1991.

Popovich JMJ, Reeves NP, Priess MC, Cholewicki J, Choi J, Radcliffe CJ.

Quantitative measures of sagittal plane head-neck control: A test-retest reliability study. $J$ Biomech 48: 549-554, 2015.

Reeves NP, Popovich JMJ, Priess MC, Cholewicki J, Choi J, Radcliffe CJ. Reliability of assessing trunk motor control using position and force tracking and stabilization tasks. $J$ Biomech 47: 44-49, 2014.

Rispens SM, van Schooten KS, Pijnappels M, Daffertshofer A, Beek PJ, van Dieën JH. Identification of fall risk predictors in daily-life measurements - gait characteristics' 
reliability and association with self-reported fall history. Neurorehabil Neural Repair 29: 5461, 2015.

Rubenstein LZ. Falls in older people: epidemiology, risk factors and strategies for prevention. Age Ageing 35 Suppl 2: ii37-ii41, 2006.

Ruhe A, Fejer R, Walker B. The test-retest reliability of centre of pressure measures in bipedal static task conditions--a systematic review of the literature. Gait Posture 32: 436445, 2010.

Safavynia S a, Ting LH. Long-latency muscle activity reflects continuous, delayed sensorimotor feedback of task-level and not joint-level error. J Neurophysiol 110: 1278-90, 2013.

Santos BR, Delisle A, Lariviere C, Plamondom A, Imbeau D. Reliability of centre of pressure summary measures of postural steadiness in healthy young adults. Gait Posture 27: 408-415, 2008.

Schouten AC, Boonstra TA, Nieuwenhuis F, Campfens SF, van der Kooij H. A Bilateral Ankle Manipulator to Investigate Human Balance Control. IEEE Trans Neural Syst Rehabil Eng 19: 660-669, 2011.

Schwab AL. Multibody Dynamics B. TU Delft, 1998.

Shadmehr R, Mussa-Ivaldi A. Adaptive Task of Dynamics during Learning of a Motor. $J$ Neurosci 14: 3208-3224, 1994.

Shavelson RJ, Webb NM. Generalizability Theory. London: SAGE Publications, 1991.

Sibley KM, Straus SE, Inness EL, Salbach NM, Jaglal SB. Clinical balance assessment: perceptions of commonly-used standardized measures and current practices among physiotherapists in Ontario, Canada. Implement Sci 8: 33, 2013.

Speers R, Kuo D, Horak FB. Contributions of altered sensation and feedback responses to changes in coordination of postural control due to aging. Gait Posture 16: 20-30, 2002.

Stijntjes M, Pasma JH, Vuuren van M, Blauw GJ, Meskers CGM, Maier AB. Low cognitive status is associated with a lower ability to maintain standing balance in elderly outpatients. Gerontology 61: 124-130, 2015.

Sturnieks DL, St George R, Lord SR. Balance disorders in the elderly. Clin Neurophysiol 38: 467-78, 2008.

Teasdale N, Simoneau M. Attentional demands for postural control: The effects of aging and sensory reintegration. Gait Posture 14: 203-210, 2001. 
Verhaegen M. Filtering and System Identification; A least square approach. New York: Cambridge University Press, 2007.

De Vet HCW, Terwee CB, Knol DL, Bouter LM. When to use agreement versus reliability measures. J Clin Epidemio/ 59: 1033-1039, 2006.

Visser JE, Carpenter MG, van der Kooij H, Bloem BR. The clinical utility of posturography. Clin Neurophysiol 119: 2424-2436, 2008.

De Vlugt E, Schouten AC, van der Helm FCT. Adaptation of reflexive feedback during arm posture to different environments. Biol Cybern 87: 10-26, 2002.

De Vlugt E, Schouten AC, van der Helm FCT. Closed-loop multivariable system identification for the characterization of the dynamic arm compliance using continuous force disturbances: a model study. J Neurosci Methods 122: 123-40, 2003.

De Vlugt E, Schouten AC, van der Helm FCT. Quantification of intrinsic and reflexive properties during multijoint arm posture. J Neurosci Methods 155: 328-49, 2006.

Weerdesteyn V, Laing AC, Robinovitch SN. The body configuration at step contact critally determines the successfulness of balance recovery in response to large backward perturbations. Gait Posture 35: 462-466, 2012.

Weir JP. Quantifying test-retest reliability using the intraclass correlation coefficient and the SEM. J Strength Cond Res 19: 231-240, 2005.

WHO. World Health Organization Global Report on Falls Prevention in Older Age [Online]. http://www.who.int/ageing/publications/Falls_prevention7March.pdf?ua=1.

Van Wingerden J-W. Control of Wind Turbines with "Smart" Rotors: Proof of Concept \& LPV Subspace Identification. : 160, 2008.

Winter D a, Patla a E, Rietdyk S, Ishac MG. Ankle muscle stiffness in the control of balance during quiet standing. J Neurophysio/ 85: 2630-3, 2001.

Winter DA, Patla AE, Frank JS. Assessment of balance control in humans. Med Prog Technol 16: 31-51, 1990.

Winter DA. Biomechanics and Motor Control of Human Movement. Second edi. Waterloo, Ontario, Canada: John Wiley and Sons, 1990.

Wrisley DM, Stephens MJ, Mosley S, Wojnowski A, Duffy J, Burkard R. Learning effects of repetitive administrations of the sensory organization test in healthy young adults. Arch Phys Med Rehabil 88: 1049-54, 2007. 


\section{Summary}

Humans are able to maintain balance in many daily life activities and under numerous environmental conditions. However, bipedal stance is inherently unstable and without a stabilizing control mechanism we would fall. Control of balance comprises a complex interplay of various underlying systems (i.e. the sensory systems, the motor system, and the nervous system), which together form the neuromuscular controller. With age, the underlying systems of the neuromuscular controller can deteriorate, which increases the risk of falling and especially the consequences of a fall become more severe. The general goal of this thesis is to create further insight in the (patho)physiology of standing balance control in elderly, by specifically developing novel and improved experimental techniques to assess the contribution of the underlying mechanisms in standing balance control.

Chapter 2 describes that available clinical balance tests determine the patient's ability to maintain standing balance under defined test conditions and aim to describe the current state of this ability. However, these tests are unable to reveal how well the neuromuscular controller is functioning and the tests have little influence on clinical decision making. Especially detection of early-stage balance control deterioration is difficult, because the balance control system is redundant and elderly may use compensation strategies; i.e. cause and effect relations are unclear. There is a clear need for new methods to identify the underlying mechanisms and compensation strategies. System identification techniques in combination with specifically designed external disturbances, may provide a way to disentangle cause and effect in balance control. By externally exciting the system with a unique input that is not related to the internal signals of the system, creates a causal relation between the external disturbances and output signals. By appropriate analysis, specific parts of the system can be identified; e.g. the neuromuscular controller dynamics under various conditions. Furthermore, a mathematical model of balance control (consisting of physiologically relevant parameters) can be fitted to the acquired data. These parameters can be compared between groups, individuals or conditions.

Differences between young and elderly often become more pronounced in multi-segmental balance control; i.e. studying the coordination between the ankles and hip joints in balance control. The application of system identification techniques in human balance control is challenging when considering multi-segmental balance control, in which the human body is modeled as a double inverted pendulum; i.e. a multiple-input-multiple-output (MIMO) system. The system must be externally excited with an independent disturbance for each degree of freedom in the MIMO system. In this thesis, force disturbances were applied at the hips and between the shoulder blades. As the field of system identification is very broad, 
with many approaches and various techniques and methods, it is far from trivial to compare the methods reported in literature, as there are always differences in experimental design, use of external disturbance signals and the results are presented in different ways.

Therefore, in Chapter 3, various existing MIMO non-parametric and parametric system identification techniques were compared, in combination with multiple designs of external force disturbances at the hips and between the shoulder blades. Comparison was based on model simulations; i.e. by simulating a two segmental balance control model that contains the dynamics of the underlying physiology, and applying force disturbances on the hips and between the shoulder blades. Validation of the techniques was based on the estimation of the neuromuscular controller dynamics in the time and frequency domain. Results indicated that non-parametric techniques with multisine disturbances gave the most accurate estimation of the neuromuscular controller mechanisms in both the time domain and frequency domain. Based on these outcomes, this system identification approach was applied in the experimental studies in Chapter 4 and Chapter 5.

In Chapter 4, a novel device was presented - the double inverted pendulum perturbator (DIPP) - which can apply disturbing forces at the hip level and between the shoulder blades. In addition to the disturbances, the device can provide force fields to study adaptation of multi-joint coordination. The performance of the DIPP and validation of the system identification approach was assessed by first identifying a system with known mechanical properties. A double inverted pendulum was successfully identified, while force fields were able to keep the pendulum upright. An experiment with healthy young subjects where a stabilizing force field was rendered at the hip, showed that humans adapted their balance control responses, by lowering their control actions around the ankles. These results imply that the DIPP in combination with system identification methods allows to study (pathological) changes in multi-joint coordination as well as adaptive capacity to maintain standing balance.

In Chapter 5, age-related changes in multi-joint balance control were studied within a population of healthy young and healthy elderly participants. Adaptation capacities were studied with external force fields, represented by an external stiffness at the hip, either stabilizing or destabilizing the participants' balance. Frequency Response Functions (FRFs) quantified the corrective joint torques as a response to body sway, from which a description of the neuromuscular control mechanisms was established. Elderly showed an increased FRF magnitude compared to young participants, suggesting that elderly were stiffer. When a stabilizing or destabilizing external force field was applied at the hip, both young and elderly participants adapted their multi-joint coordination by lowering or respectively increasing their neuromuscular control actions around the ankles; expressed in a change of FRF magnitude. However, elderly adapted less compared to young participants. Model fits showed that elderly had comparable reflexive stiffness and reflexive damping properties to the young, but they adapted their reflexive stiffness around the ankle joint less. Furthermore, elderly showed higher intrinsic stiffness of the ankle, together with higher time 
delays of the hip. These age related differences in adaptation of multi-joint coordination may underlie increased risk of falling with ageing.

These results show that system identification techniques can assess age related changes in standing balance control. However, before introducing the method into clinical practice for diagnosing or monitoring treatment of impaired balance, it is important to assess the reliability of this technique and compare it with current clinical balance measures. In addition to the force disturbances at the hip and between the shoulder blades, two sensory disturbances were applied on the proprioceptive system and the visual system. This allowed not only to study multi-segmental balance control, but also the participants' reliance on sensory information (sensory reweighting).

In Chapter 6, the reliability of standing balance parameters was studied, when multiple disturbances were applied simultaneously (i.e. two mechanical disturbances at the hip and shoulder and two sensory disturbances of proprioceptive and visual information) and with system identification techniques to assess standing balance in healthy elderly. The reliability was compared with a parameter that represents the amount of body sway, often looked at in clinical balance tests. Results were obtained by measuring balance parameters twice during three days.

A systematic error was found in the sensitivity functions, obtained with system identification techniques, as the balance parameters differed between the first and second trial on one day. No systematic error was found in the neuromuscular controller parameters. Therefore, when a steady state of standing balance is to be assessed with system identification techniques, a familiarization trial is needed on the same day to overcome the systematic error between trials. The balance parameters are reliable; i.e. the parameter could detect changes over time within the same participant (e.g. effects of intervention or changes in conditions). The results showed that at least 10 trials on one day are needed to reach an excellent reliability for steady state balance assessment. No systematic errors and a higher reliability was found for the body sway parameter. However, the body sway parameters only describes standing balance objectively, while system identification techniques also describe the underlying changes. Therefore, the use of system identification techniques can have added value over current clinical balance measures, such as body sway.

This thesis shows that the application of external disturbances in combination with system identification techniques give more insight in the underlying physiology of balance control and the changes with age. Using the techniques as a diagnostic tool, can help to detect impaired balance and ultimately reduce the consequences of falls in the elderly. 


\section{Samenvatting}

In het dagelijks leven handhaven mensen hun balans tijdens het uitvoeren van verschillende taken en onder verschillende omstandigheden. De zwaartekracht trekt voortdurend aan het lichaam, waardoor er een constante instabiele situatie ontstaat. Zonder een goed regelmechanisme zouden we vallen. Bij balanshandhaving zijn meerdere onderliggende systemen betrokken, zoals de sensorische systemen, de spieren en het zenuwstelsel. Deze systemen vormen samen een complex regelmechanisme die het lichaam rechtop houdt; dit wordt de neuromusculaire regelaar genoemd. Door het verouderingsproces kunnen de onderliggende systemen van de neuromusculaire regelaar in kwaliteit achteruit gaan, waardoor het risico op vallen toeneemt en vooral de gevolgen van een val groter worden. Het doel van dit proefschrift is om inzicht te krijgen in de (pathologische) fysiologie van balanshandhaving bij ouderen, door het ontwikkelen van nieuwe en verbeterde experimentele technieken die de bijdrage van de onderliggende systemen in balanshandhaving kunnen beschrijven.

Hoofdstuk 2 beschrijft dat de huidige klinische balans maten weliswaar testen of een patiënt onder verschillende omstandigheden in staat is rechtop te blijven staan, maar er wordt geen onderscheid gemaakt in de kwaliteit en bijdrage van de onderliggende systemen van de neuromusculaire regelaar. Deze testen hebben weinig tot geen invloed op het voorschrijven van een doelgerichte behandeling. Het is voornamelijk lastig om problemen met de balanshandhaving in een vroeg stadium te detecteren, omdat ouderen om rechtop te blijven staan vaak compensatie strategieën gebruiken; daardoor zijn oorzaak en gevolg niet duidelijk. Nieuwe onderzoeksmethodes zijn nodig die zowel de kwaliteit van de onderliggende systemen als de aanwezige compensatie strategieën in kaart kunnen brengen. Systeem identificatie technieken in combinatie met extern aangebrachte verstoringen, kunnen de mogelijkheid bieden om de oorzaak en gevolg relaties bij balanshandhaving te ontrafelen. Door het menselijke balanssysteem te verstoren met een uniek signaal, dat niet gerelateerd is aan de interne signalen van het systeem, ontstaat een causale relatie tussen de extern aangebrachte verstoringen en de menselijke reacties. Hierdoor is het mogelijk om, met de juiste analyse methodes, specifieke delen van het regelmechanisme van de balanshandhaving te identificeren; zoals het gedrag van de neuromusculaire regelaar tijdens verschillende experimentele condities. Vervolgens kan er een model van balans handhaving (bestaande uit fysiologische relevante parameters) gefit worden op de verkregen data. Deze parameters kunnen dan vergeleken worden tussen groepen, individuen of condities. 
Verschillen tussen jongeren en ouderen komen vaak beter naar voren als er gekeken wordt naar de coördinatie tussen het enkel en heup gewricht; ofwel multi-segmentale balanshandhaving. Het toepassen van systeem identificatie technieken op multi-segmentale balanshandhaving biedt een aantal uitdagingen. De mens wordt allereerst voorgesteld als een dubbele omgekeerde slinger. Dit resulteert in een systeem met meerdere ingangen en meerdere uitgangen (MIMO systeem). Voor elke ingang moet er een aparte externe verstoring worden aangebracht om het gehele systeem goed te kunnen identificeren. In dit proefschrift zijn krachtsverstoringen gebruikt op de heup en tussen de schouderbladen. Verder is het onderzoeksgebied van systeem identificatie erg breed. Het is niet eenvoudig om deze methodes met elkaar te vergelijken op basis van literatuur onderzoek, omdat de studies verschillend in de opzet van de experimenten, het gebruik van de externe verstoorsignalen, en de resultaten zijn vaak op verschillende manieren gepresenteerd.

Daarom zijn in Hoofdstuk 3, verschillende MIMO niet-parametrische en parametrische systeem identificatie technieken met elkaar vergeleken, in combinatie met het gebruik van verschillende externe verstoorsignalen op de heup en tussen de schouderbladen. De vergelijking is gebaseerd op model simulaties, waarbij het menselijke balans systeem is gesimuleerd als een dubbele omgekeerde slinger die gebalanceerd wordt door een neuromusculaire regelaar. De onderliggende dynamica komt overeen met fysiologische waardes en er worden krachtsverstoringen aangebracht op de heup en tussen de schouder bladen. Validatie van de systeem identificatie technieken is gebaseerd op een schatting van de neuromusculaire regelaar dynamica in het tijd domein en in het frequentie domein. Resultaten laten zien dat de beste schatting verkregen wordt met niet-parametrische systeem identificatie technieken in combinatie met multisinus verstoringen. Gebaseerd op deze uitkomsten, zijn de systeem identificatie technieken toegepast in de studies van Hoofdstuk 4 en Hoofdstuk 5.

Hoofdstuk 4 introduceert een nieuw apparaat - de dubbele omgekeerde slinger verstoorder (double inverted pendulum perturbator, afgekort DIPP) - die krachtsverstoringen kan aanbieden op de heup en tussen de schouder bladen. Naast de externe verstoringen, kan het apparaat ook krachtvelden aanbieden, waarmee het mogelijk is om het aanpassingsvermogen van multi-segmentale balanshandhaving te onderzoeken. De prestaties van het apparaat en de validatie van de systeem identificatie techniek is eerst onderzocht door het identificeren van de dynamica van een bekend systeem. Een dubbele omgekeerde slinger is succesvol geïdentificeerd, terwijl de krachtvelden de slinger in evenwicht hielden. Een experiment met gezonde jongeren laat zien, wanneer er een krachtveld op de heup aangebracht wordt, dat mensen hun balanshandhaving aanpasten. De regelactie rondom het enkel gewricht werd minder. Deze resultaten laten zien dat de DIPP samen met het gebruik van systeem identificatie technieken het mogelijk maakt om (patho)fysiologische veranderingen in multi-segmentale balanshandhaving te onderzoeken. Daarnaast kan het aanpassingsvermogen in balanshandhaving bestudeerd worden.

In Hoofdstuk 5 zijn binnen een populatie van gezonde jongeren en gezonde ouderen, veranderingen door leeftijd in de multi-segmentale balanshandhaving onderzocht. 
Aanpassingsvermogen wordt bestudeerd met behulp van externe krachtvelden (een externe stijfheid op de heup), die de balans stabiliseerde, of destabiliseerde. Frequentie Responsie Functies (FRFs) geven de relatie weer tussen de corrigerende gewrichtsmomenten als een reactie op lichaamsbewegingen, wat resulteerde in een identificatie van de dynamica van de neuromusculaire regelaar. Ouderen hadden een hogere FRF magnitude vergeleken met jongeren, wat suggereert dat ouderen stijver zijn. Wanneer er een stabiliserend of destabiliserend krachtveld werd aangeboden op de heup, pasten zowel de jongeren als de ouderen hun balanshandhaving hierop aan. De neuromusculaire regelaar acties rond de enkel werden verhoogd of verlaagd, wat te zien was in de verandering van FRF magnitude. Echter, ouderen pasten zich minder aan op de krachtvelden dan de jongeren. Model fits op de FRF lieten zien dat ouderen vergelijkbare reflexieve stijfheid en demping eigenschappen hadden vergeleken met de jongeren, maar dat zijn hun reflexieve stijfheid rond de enkel minder aanpasten. Daarnaast hadden ouderen een hogere intrinsieke stijfheid van de enkel en hogere tijdsvertraging van de heup. Deze veranderingen met leeftijd in het aanpassingsvermogen van multi-segmentale balanshandhaving, kunnen ten grondslag liggen aan het toegenomen valrisico bij ouderen.

Bovenstaande resultaten impliceren dat het mogelijk is om verschillen te detecteren in balanshandhaving met leeftijd, door gebruik te maken van systeem identificatie technieken. Desalniettemin, voordat de technieken geïmplementeerd kunnen worden in de klinische praktijk als diagnostisch middel of voor het monitoren van het effect van behandeling, is het noodzakelijk de betrouwbaarheid van de techniek te onderzoeken. Deze betrouwbaarheid kan dan vergeleken worden met bestaande klinische balans maten. Naast de krachtverstoringen op de heup en tussen de schouderbladen, zijn twee sensorische verstoringen aangebracht op het proprioceptieve en visuele systeem. Hiermee werd het niet alleen mogelijk om multi-segmentale balanshandhaving te onderzoeken, maar ook de betrouwbaarheid en gebruik van sensorische informatie (sensorische herweging).

In Hoofdstuk 6 is de betrouwbaarheid van balans parameters onderzocht, wanneer er meerdere verstoringen tegelijk werden aangebracht (twee krachtverstoringen op de heup en tussen de schouderbladen, en twee sensorische verstoringen van de proprioceptie en de visus) en in combinatie met systeem identificatie technieken. Balanshandhaving werd onderzocht in een populatie van gezonde ouderen. De betrouwbaarheid werd vergeleken met een parameter die vaak als klinische maat gebruikt wordt, namelijk de lichaamszwaai. Betrouwbaarheid is bepaald op basis van twee metingen op drie dagen.

De balans parameters die verkregen zijn met systeem identificatie technieken op basis van de sensorische verstoringen bevatten een systematische fout. De parameters verschillen tussen de eerste en tweede meting op een dag. In de neuromusculaire regelaar parameters (gebaseerd op de krachtverstoringen) is geen systematische fout aanwezig. Om systematische fouten te voorkomen, kan een gewenningsmeting worden uitgevoerd aan het begin van een dag. De balans parameters zijn betrouwbaar, wat betekent dat er verschillen binnen de proefpersoon gedetecteerd kunnen worden (bijvoorbeeld het effect van interventies of verschillende experimentele condities). Resultaten laten zien dat minimaal 10 
metingen op een dag nodig zijn om een uitstekende betrouwbaarheid te krijgen. De lichaamszwaai parameter bevat geen systematische fout en heeft daarnaast ook een hogere betrouwbaarheid. Desalniettemin, beschrijt de lichaamszwaai parameter geen onderliggende veranderingen van de neuromusculaire regelaar en kan het gebruik van systeem identificatie technieken een toegevoegde waarde hebben over bestaande klinische balans maten.

In dit proefschrift is beschreven dat het gebruik van systeemidentificatie technieken, in combinatie met externe verstoringen meer inzicht geven in de onderliggende fysiologie van balanshandhaving en de verschillen die kunnen ontstaan door ouderdom. Het gebruik van de technieken als een diagnostisch middel, kan helpen om verminderde balanshandhaving te detecteren en uiteindelijk de gevolgen van vallen te verkleinen. 


\section{Acknowledgements}

$\mathrm{Na}$ een ruime vier jaar is de tijd gekomen om de laatste hand te leggen aan mijn proefschrift en het 'luchtigste' deel, namelijk het dankwoord, te schrijven. Ik wil hier graag de mensen benoemen die de afgelopen jaren hebben bijgedragen aan het tot stand komen van mijn proefschrift.

Allereerst mijn drie directe begeleiders die mij tijdens het hele promotietraject hebben bijgestaan. Herman, jouw kritische blik heeft de inhoud van het proefschrift naar een hoger niveau gebracht. Ondanks dat je direct in het begin van mijn promotie naar Zwitserland ging voor een sabbatical, is je betrokkenheid bij het project nooit minder geweest. De vrijheid die je geeft om op eigen wijze het onderzoek uit te voeren, heb ik enorm gewaardeerd. Bedankt ook voor alle opbouwend kritiek op de eindeloze stroom documenten die ik jouw kant op heb gestuurd.

Alfred, samen hebben we heel wat uren in het lab doorgebracht; bouwen, klussen, inregelen en verhuizen. Met jouw enthousiasme heb je mijn liefde voor techniek versterkt. Ondanks dat je maar eens per twee weken in Twente was, vond je toch tijd om samen aan artikelen te schrijven onder het genot van een kop koffie. Ook bedankt voor de relativerende woorden als ik die nodig had en waardoor ik alles weer wat zonniger ging inzien.

Ronald, onze treinreisjes zijn memorabel vanwege het debuggen van de Matlab scripts. Ondanks jouw drukke agenda wist je altijd tijd vrij te maken voor mijn systeemidentificatie vragen. Met een aantal grote beschreven bladen oud-printer papier, vol nieuwe ideeën en met hervonden enthousiasme kwam ik jouw kantoor uit. Bedankt voor de fijne samenwerking de afgelopen jaren.

Het BalRoom project had ook geen kans van slagen gehad zonder mijn zeer gewaardeerde collega en goed vriendin Jantsje. Samen begonnen met de studie Biomedische Technologie, binnen hetzelfde project gepromoveerd en elkaars paranimf. Samen hebben we een hele hoop werk verzet om dit project tot een goed einde te brengen. Ik heb genoten van alle emails waarin werk en privé regelmatig door elkaar liepen. Zonder jouw hulp en bijdrage was het promoveren lang niet zo leuk geweest!

Voor het tot stand komen van de experimentele studies (al dan niet met ouderen), gaat alle dank uit naar de projectpartners vanuit Leiden (en later Amsterdam). Carel en Andrea, wat fijn dat jullie te allen tijde de belangen van de ouderen in het vizier hebben gehouden en de relevantie van de BalRoom gepromoot hebben. 
De BalRoom hardware is tot stand gekomen in nauwe samenwerking met ForceLink, waar ik mij gedurende de jaren altijd welkom heb gevoeld. Peter, dank je wel voor alle uren en noeste arbeid die in de bouw van de BalRoom zijn gaan zitten. Dat je kans van slagen zag als diagnostisch apparaat, motiveerde mij om de experimentele studies uit te voeren. Het zou toch mooi zijn als we later zelf oud zijn, (hopelijk geen) valproblematiek hebben, we in de 'badkuip' kunnen staan.

Daarnaast wil ik de collega's uit het NeurSIPE programma bedanken, voor de interessante summerschools (in de winter) en de leerzame en leuke tijd in New Orleans en Chicago.

Tijdens mijn promotie had ik de eer te mogen werken met een heleboel collega's van de vakgroep Biomedische Werktuigbouwkunde. De ruimte is hier helaas te kort om iedereen te noemen, maar ik wil jullie allemaal bedanken voor de leuke tijd op congressen, tijdens vakgroepsuitjes, in de koffiepauze, potlucks, lunchwandelingen en sportdagen. Toch wil ik graag een aantal mensen in het bijzonder bedanken.

Met Vera, als harde kern van het kantoor HR-W216, zaten we met de voetjes op de vensterbank het 'leven' te analyseren. Allerhande onderwerpen hebben we besproken; de review commentaren, de belevenissen tijdens metingen en de mensen in de fietsenstalling. Bedankt dat je me ook in dit laatste stukje van het promotietraject wilt bijstaan als paranimf. Claudia, Mark en Hielke, jullie ook bedankt voor de nodige pauzes in de koffiecorner (al dan niet met uithuil-muffins), de fietstochten, etentjes en andere uitjes buiten werk om. Ik vond het erg leuk om met jullie samen te werken, lief en leed te delen, en kijk met erg veel plezier terug op de afgelopen jaren. We houden contact!

Tjitske, jouw promotietraject was bijna ten einde toen ik jouw 'opvolger' werd. Je bent gedurende het traject een sparring-partner geweest en ik kon altijd bij jou terecht voor vragen. Ik ben jou als ervaren wetenschapper vooral dankbaar voor de coaching op de planning van het onderzoek en dat je me wijze raad en advies hebt gegeven op tijden dat ik dat nodig had. Onze onderzoeken zijn mooi samen gekomen in het MIMO de FLIMO paper, dat er zonder jouw hulp niet was gekomen.

Wouter en Nikolai, bedankt voor alle technische ondersteuning tijdens mijn onderzoek. Zeer regelmatig moest er ad-hoc een BalRoom onderdeel vervangen worden en dan stonden jullie direct voor mij klaar. Vooral de verhuizing van de apparatuur heb ik echt als een avontuur ervaren!

Lianne, waar zouden we zijn zonder jouw organisatorisch vermogen? Daarnaast heb je een luisterend oor paraat; goed nieuws of slecht nieuws, werk-gerelateerd of privé. Ik wil je ontzettend bedanken voor het feit dat jouw deur altijd open staat.

Dit werk had niet tot stand kunnen komen, zonder de welwillendheid van alle proefpersonen om mee te werken aan mijn onderzoek. Belangeloos hebben zij zich ingezet om plaats te nemen in (delen van) de BalRoom. Ook voorafgaand aan de daadwerkelijke studie was jullie hulp zeer nodig, als ik weer 'even' wat pilot meetresultaten nodig had. Bedankt voor jullie enthousiasme en nieuwsgierigheid die mij de relevantie van het onderzoek lieten inzien. 
Zeker niet onbelangrijk zijn mijn vrienden en familie, die mij thuis (al dan niet bewust) hebben ondersteund en gemotiveerd om het promotietraject te kunnen afronden. Bedankt dat jullie het rotsvaste geloof hadden dat het einde in zicht zou komen, terwijl ik dat niet altijd zag. In het bijzonder toch een dankwoord voor pap en mam. Regelmatig ver weg op reis, maar altijd dichtbij. Zonder jullie steun, levenservaring en goede adviezen had ik het niet gered.

Lieve Jeroen, wij hebben bewezen dat apart wonen je ook dichterbij elkaar kan brengen. Jouw nuchtere kijk op het leven laat mij ontspannen en lachen. Bijzonder vind ik jouw vanzelfsprekende vertrouwen in ons samen, en ik kijk met plezier uit naar alles wat de toekomst ons brengt. Ik houd van je!

Denise van der Meer - Engelhart 


\section{About the author}

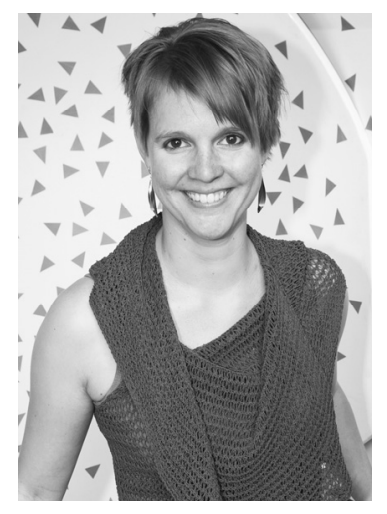

Denise van der Meer - Engelhart was born in Zwolle, The Netherlands, on $4^{\text {th }}$ of December 1986. She started her bachelor Biomedical Engineering in 2004 at the University of Twente, Enschede, The Netherlands. During her bachelor assignment, she designed an alternative pedicle screw for scoliosis correction. This was followed by a minor Business Administration and an internship at the Centre for Man in Aviation, Soesterberg, The Netherlands where Denise developed a method to measure muscle activity during straining maneuvers in a human centrifuge.

During her master, she developed an interest in human movement control and scientific research. In 2011 she received the M.Sc. degree in Biomedical Engineering from the University of Twente, Enschede, The Netherlands on the topic of lateral balance control during walking and the prediction of foot placement.

Following the work at the Biomechanical Engineering group, Denise started her PhD study in the NeuroSIPE BalRoom project. Her research focused on using system identification techniques to study and quantify human balance control. She applied these new and adopted methods in a healthy young and a healthy elderly population. A close collaboration existed with the Leiden University Medical Centre, Leiden, the Netherlands.

Since $1^{\text {st }}$ of April 2015, Denise works as a lecturer at the Rotterdam University of Applied Sciences, Rotterdam, the Netherlands, within the educational program of Healthcare Technology. 


\section{Journal publications}

Denise Engelhart, Jantsje H. Pasma, Alfred C. Schouten, Carel G.M. Meskers, Andrea B. Maier, Thomas Mergner, Herman van der Kooij. Impaired Standing Balance in Elderly: A New Engineering Method Helps to Unravel Causes and Effects. JAMDA 15 (2014) 227.e1227.e6

J.H. Pasma, D. Engelhart, A.C. Schouten, H. van der Kooij, A.B. Maier, C.G.M. Meskers. Impaired standing balance: The clinical need for closing the loop. Neuroscience 267 (2014) 157-165

D. Engelhart, A.C. Schouten, R.G.K.M. Aarts, H. van der Kooij. Assessment of multi-joint coordination and adaptation in standing balance: a novel device and system identification technique. IEEE TNSRE (2014) Epub ahead of print

D. Engelhart, J.H. Pasma, A.C. Schouten, R.G.K.M. Aarts, C.G.M. Meskers, A.B. Maier, H. van der Kooij. Adaptation of multi-joint coordination during standing balance in healthy young and healthy old individuals. Submitted

J.H. Pasma, D. Engelhart, A.B. Maier, A.C. Schouten, H. van der Kooij, C.G.M. Meskers. Changes in sensory weighting and reweighting of proprioceptive information during standing balance with age and disease. Submitted

Tjitske A. Boonstra, Joost van Kordelaar, Denise Engelhart, Jeroen P.P. van Vugt and Herman van der Kooij. Asymmetries in reactive and anticipatory balance control are of similar magnitude in Parkinson's disease patients. Submitted

D. Engelhart, T.A. Boonstra, R.G.K.M. Aarts, A.C. Schouten and H. van der Kooij. Comparison of closed loop system identification methods to quantify multi-joint human balance control. Submitted

J.H. Pasma*, D. Engelhart*, A.C. Schouten, R.G.K.M. Aarts, C.G.M. Meskers, A.B. Maier, H. van der Kooij. Reliability of system identification techniques to assess standing balance in healthy elderly. In preparation

D.Engelhart*, I. Schut*, R.G.K.M. Aarts, H. van der Kooij and A.C. Schouten. The effect of compliant support surfaces on reweighting of proprioceptive information. In preparation

*Both authors contributed equally 


\section{Conference contributions}

D. Engelhart, J.H. Pasma, A.C. Schouten, R.G.K.M. Aarts, A.B. Maier, C.G.M. Meskers, H. van der Kooij. The Balance Test Room: for diagnosis of impaired balance in elderly. MIRA institute for Biomedical Technology and Technical Medicine biannual conference, November 2014, Enschede, The Netherlands.

D. Engelhart, J.H. Pasma, A.C. Schouten, R.G.K.M. Aarts, A.B. Maier, C.G.M. Meskers, H. van der Kooij. The Balance Test Room: for diagnosis of impaired balance in elderly. STW annual conference, October 2014, Utrecht, The Netherlands.

A.C. Schouten, D. Engelhart, R.G.K.M. Aarts, J.H. Pasma, A.B. Maier, C.G.M. Meskers, H. van der Kooij. Unraveling causes and effects in human balance control: the BALROOM approach. 36th Annual International Conference of the IEEE Engineering in Medicine and Biology Society. August 2014, Chicago, USA

D. Engelhart, A.C. Schouten, R.G.K.M. Aarts, J.H. Pasma, C.G.M. Meskers, A.B. Maier, H. van der Kooij. Adaptation of multi-joint balance coordination to whole body force fields. International Society for Posture and Gait Research conference, June 2014, Vancouver, Canada

J.H. Pasma, D. Engelhart, A.C. Schouten, A.B. Maier, C.G.M. Meskers, H. van der Kooij. Sensory reweighting of proprioceptive input during balance control as function of age and disease. International Society for Posture and Gait Research conference, June 2014, Vancouver, Canada

D. Engelhart, A.C. Schouten, R.G.K.M. Aarts, H. van der Kooij. Less is more: efficient identification of human stance control with parametric subspace identification. $4^{\text {th }}$ Dutch BME conference, January 2013, Egmond aan Zee, The Netherlands

J.H. Pasma, D. Engelhart, A.B. Maier, A.C. Schouten, C.G.M. Meskers, H. van der Kooij. Sensory reweighting of proprioceptive input during balance control in healthy elderly. $4^{\text {th }}$ Dutch BME conference, Januari 2013, Egmond aan Zee, The Netherlands

D. Engelhart, A.C. Schouten, R.G.K.M. Aarts, H. van der Kooij. Less is more: efficient identification of human stance control with parametric subspace identification. Society for NeuroScience conference, October 2012, New Orleans, USA.

J.H. Pasma, D. Engelhart, A.B. Maier, A.C. Schouten, C.G.M. Meskers, H. van der Kooij. Sensory reweighting of proprioceptive input during balance control in healthy elderly. Society for NeuroScience conference, October 2012, New Orleans, USA.

D. Engelhart, E.H.F. van Asseldonk, L. Grin, H. van der Kooij. Lateral balance control during walking: Foot placement and stability in response to external perturbations. International Society for Posture and Gait Research conference, June 2012, Trondheim, Norway 
D. Engelhart, E.H.F. van Asseldonk, L. Grin, H. van der Kooij. Lateral balance control during walking: Can the XcoM model predict foot placement during lateral external perturbations. $6^{\text {th }}$ Posture symposium, September 2011, Smolenice, Slovakia 
